\title{
QCD FITS TO NEUTRINO-IRON STRUCTURE FUNCTIONS AT NUTEV
}

\author{
by \\ Voica A. Radescu \\ MS in Physics, University of Pittsburgh, 2001
}

Submitted to the Graduate Faculty of

the Department of Physics and Astronomy in partial fulfillment

of the requirements for the degree of

Doctor of Philosophy

University of Pittsburgh

2006 


\section{UNIVERSITY OF PITTSBURGH}

\section{DEPARTMENT OF PHYSICS AND ASTRONOMY}

This dissertation was presented

by

Voica A. Radescu

It was defended on

August 3, 2006

and approved by

Dr. Donna Naples, Associate Professor, Dept. of Physics and Astronomy Dr. Paul Shepard,Professor, Dept. of Physics and Astronomy

Dr. Vittorio Paolone, Associate Professor, Dept. of Physics and Astronomy

Dr. Adam Leibovich, Assistant Professor, Dept. of Physics and Astronomy

Dr. Rob Coalson, Professor, Dept. of Chemistry/Dept. of Physics and Astronomy

Dissertation Director: Dr. Donna Naples, Associate Professor, Dept. of Physics and

Astronomy 


\title{
ABSTRACT \\ QCD FITS TO NEUTRINO-IRON STRUCTURE FUNCTIONS AT NUTEV
}

\author{
Voica A. Radescu, PhD
}

University of Pittsburgh, 2006

This thesis presents a new determination of $\Lambda_{Q C D}$ from Next-to-Leading Order QCD fits to the $Q^{2}$ dependence of neutrino-iron structure functions. This is the first measurement of $\Lambda_{Q C D}$ which uses a theoretical model that fully accounts for heavy quark production. Compared with previous neutrino measurements, the result has improved understanding of the largest systematic uncertainties on the muon and hadron energy scales. These improvements lead to one of the most precise determination of $\alpha_{S}$ at moderate $Q^{2}$.

$\mathrm{NuTeV}$ is a neutrino-iron deep inelastic scattering (DIS) experiment that collected data during 1996-97 at Fermilab. The key features of NuTeV include its sign-selected beam which produced separate high purity neutrino and antineutrino beams, and its continuous calibration beam which enabled $\mathrm{NuTeV}$ to considerably improve the knowledge of energy scales which have dominated uncertainties in the previous measurements. 


\section{TABLE OF CONTENTS}





1.1 Standard Model and Elementary Particles . . . . . . . . . . . . . . . . 2

1.2 Deep Inelastic Scattering and Quark Parton Model . . . . . . . . . . . . . 3

1.2.1 Kinematics of $\nu-N$ Deep Inelastic Scattering . . . . . . . . . . . . 3

1.2.2 Structure Functions in The Quark Parton Model . . . . . . . . . . 10

1.3 Quantum Chromodynamics .................... 14

1.3.1 Perturbative QCD and $\alpha_{S} \ldots \ldots \ldots \ldots$

1.3.1.1 Renormalization . . . . . . . . . . . . . . 15

1.3.1.2 Running of $\alpha_{S} \ldots \ldots \ldots \ldots \ldots$

1.3.1.3 $\Lambda_{Q C D}$ parameter . . . . . . . . . . . . 18

1.3.1.4 Quark Thresholds ................. 19

1.3.1.5 Factorization ..................... 20

1.3.2 QCD Theory Models ... . . . . . . . . . . . . 21

1.3.2.1 Massless $\overline{M S}$ Scheme . . . . . . . . . . . . . . . . . 21

1.3.2.2 ACOT Scheme . . . . . . . . . . . . . 23

1.3.3 Evolution and QCD Sum Rules ... . . . . . . . . . . . 24

1.3.4 Non-Perturbative QCD Effects . . . . . . . . . . . 28

1.3.4.1 Target Mass . . . . . . . . . . . . . . . . . . 28

1.3.4.2 Higher-Twist . . . . . . . . . . . . . . . 30

1.3.4.3 Nuclear Target . . . . . . . . . . . . . . . 31

2.0 NUTEV EXPERIMENT . . . . . . . . . . . . . . . . . . . . 33 
$2.1 \mathrm{NuTeV}$ Detector . . . . . . . . . . . . . . . . . . . 34

2.1.1 Target Calorimeter . . . . . . . . . . . . . . . 34

2.1.2 Muon Spectrometer . . . . . . . . . . . . . . . . . 38

$2.2 \mathrm{NuTeV}$ Beamline . . . . . . . . . . . . . . . . . . . . . . 41

2.3 Data Acquisition and Event Triggering . . . . . . . . . . . . . 45

2.3.1 Readout Electronics . . . . . . . . . . . . . . . . . 46

2.3.2 Event Triggers . . . . . . . . . . . . . . . . . . . . . . . . 48

2.4 Event Reconstruction . . . . . . . . . . . . . . . . . 50

$2.4 .1 \quad$ Pulse Height . . . . . . . . . . . . . . . . . . . . . 50

2.4.2 Raw Event Variables . . . . . . . . . . . . . . . . . . . . 53



2.5.1 In Situ Calibration Beam _. . . . . . . . . . . . . . . . 59

2.5.2 Calorimeter Calibration . . . . . . . . . . . . . . . 62

2.5.3 Toroid Response Calibration . . . . . . . . . . . . . . . 67

2.5.4 Muon Angle Calibration . . . . . . . . . . . . . . . . . . . . . 69

3.0 EXTRACTION OF THE DIFFERENTIAL CROSS SECTION . . . . 71

3.1 Data Selection . . . . . . . . . . . . . . . . . 71

3.1.1 Fiducial-Volume Cuts . . . . . . . . . . . . . . . . . 72

3.1 .2 Geometric Cuts . . . . . . . . . . . . . . . . . 73

3.1 .3 Data Samples . . . . . . . . . . . . . . . . 74

3.1.3.1 Flux sample . . . . . . . . . . . . . . . . . 74

3.1.3.2 Cross section sample . . . . . . . . . . . . . . . 74

3.2 Monte Carlo Simulation . . . . . . . . . . . . . . . 75

3.2.1 Cross-Section Model . . . . . . . . . . . . . . 76

3.2.1.1 Parametrization . . . . . . . . . . . . . . 77

3.2.1.2 Physical Corrections . . . . . . . . . . . . . . 80

3.2.1.3 Cross Section Fit Results . . . . . . . . . . . . 86

3.2.2 Neutrino Event Generation _. . . . . . . . . . . . . . . . 86

3.2.3 Detector Model . . . . . . . . . . . . . . . . . . . . 88

3.3 Neutrino Flux Extraction . . . . . . . . . . . . . . . . . 90 
3.3.1 Fixed $E_{\text {had }}$ Method . . . . . . . . . . . . . . . . . . . 93

3.3.2 Flux Extraction . . . . . . . . . . . . . . . . . . . 94

3.3.3 Flux normalization . . . . . . . . . . . . . . . . . . 95

3.4 Differential Cross-Section Extraction . . . . . . . . . . . . . . . . . 96

$3.4 .1 \quad$ Iteration Procedure . . . . . . . . . . . . . . . . . . 97

3.5 Flux and Cross Section Systematic Uncertainties . . . . . . . . . . . . 100

3.6 Structure Functions Extraction . . . . . . . . . . . . . . . . . . 101

3.6.1 From 1-Parameter Fit . . . . . . . . . . . . . . . . . . . 102

3.6.2 From 2-Parameter Fit . . . . . . . . . . . . . . . . . 103

3.6.3 Structure Functions Systematic Uncertainties . . . . . . . . . . . . 104

$4.0 \Lambda_{Q C D}$ FROM NLO QCD FITS . . . . . . . . . . . . . . 105



4.1.1 PDF Parametrization and Evolution . . . . . . . . . . . . 107



4.1.2.1 Target Mass Correction . . . . . . . . . . . . . . . . 109

$4.1 .3 \quad \chi^{2}$ Minimization . . . . . . . . . . . . . . . . 109

4.1.3.1 No Correlations Case . . . . . . . . . . . . . . . . . . 110

4.1.3.2 Point-to-Point Correlations Case . . . . . . . . . . . . . . . 112

4.1.3.3 Correlation between $F_{2}$ and $x F_{3}$ Case . . . . . . . 113

$4.2 \Lambda_{Q C D}$ from NLO QCD Fits to Structure Functions . . . . . . . . . . . 114

4.2.1 Structure Function Covariance Matrix . . . . . . . . . . . . . . . . 114

4.2.2 Non-Singlet Fit Results . . . . . . . . . . . . . . . . . . . . . . . 116

4.2.3 Combined $F_{2}$ and $x F_{3}$ Fit Results . . . . . . . . . . 117

4.2.4 Comparison between Non-Singlet and Combined Fits . . . . . . . . 119

4.2.5 $\Lambda_{Q C D}$ Systematic Uncertainties . . . . . . . . . . . . . . 120

4.2.6 Theoretical Uncertainties . . . . . . . . . . . . . . . . . 124

4.2.6.1 Scale Dependence . . . . . . . . . . . . . . . . . . . 124

4.2.6.2 Quark Masses Dependence . . . . . . . . . . . 126

4.2 .7 Relate $\Lambda_{Q C D}$ to $\alpha_{S} \ldots \ldots \ldots \ldots$. . . . . . . . . . . . 128

4.3 Result Bias Study . . . . . . . . . . . . . . . . . . . . . . . 129 
4.3.1 PDF Parametrization . . . . . . . . . . . . . . . . 129

4.3.2 Kinematic Cut Dependence: $x \ldots \ldots$. . . . . . . . . . . 130

4.3.3 Kinematic Cut Dependence: $Q^{2} \ldots \ldots$. . . . . . . . . . . 130

4.3.4 Effects of Target Mass Correction . . . . . . . . . . . . . . . 131

4.4 Comparisons of NuTeV $\alpha_{S}$ to Other Measurements . . . . . . . . . . . 135

$4.4 .1 \alpha_{S}$ from DIS . . . . . . . . . . . . . . 135

4.4.1.1 Comparison with CCFR Results . . . . . . . . . . . 137

$4.4 .2 \alpha_{S}$ from Other Processes . . . . . . . . . . . . . . . 139



APPENDIX A. THE NUTEV COLLABORATION . . . . . . . . . . . . 143

APPENDIX B. STRUCTURE FUNCTIONS TABLES . . . . . . . . . . . . 144

B.1 Data Table for Non-Singlet Fit . . . . . . . . . . . . . . . . . . . . . . 144

B.2 Data Table for Combined Fit . . . . . . . . . . . . . . . . . . 146

APPENDIX C. QCD SOURCE CODE: PART A . . . . . . . . . . . . . 148

APPENDIX D. QCD PROGRAM: FITTER, EVOLUTION, $\overline{M S}$. . . . . 150

APPENDIX E. QCD SOURCE CODE: PART B . . . . . . . . . . . . 168

APPENDIX F. FIT RESULTS . . . . . . . . . . . . . . . . . . . 183

F.1 Correlation tables . . . . . . . . . . . . . . . . . . 183

F.1.1 Non-Singlet Fit . . . . . . . . . . . . . . . . . . . 183

F.1.2 Combined Fit . . . . . . . . . . . . . . . . . . 184

F.2 Nuclear PDFs from fit to $\mathrm{NuTeV}$ data . . . . . . . . . . . . . . . . 185

APPENDIX G. TESTS OF THE QCD PROGRAM . . . . . . . . . . . . 187

G.1 Test of Minimization Using CCFR Data . . . . . . . . . . . . . 187

G.1.1 CCFR vs NuTeV . . . . . . . . . . . . . . . . . . . . . 189

G.2 Test of the fitter with correlations . . . . . . . . . . . . 190

G.3 Test of the fusion . . . . . . . . . . . . . . . . . . 191

G.3.1 Fake data . . . . . . . . . . . . . . . . . . . . . . . . 191

G.3.2 Comparison between Massless $\overline{M S}$ Models . . . . . . . . . . . . . . 191

G.3.3 ACOT Technicalities . . . . . . . . . . . . . . . . 192

G.4 ACOT and Its Variant . . . . . . . . . . . . . . . . . . 193 


\section{LIST OF TABLES}

1.1 Fundamental forces described in the Standard Model. . . . . . . . . . . . . . 3

1.2 Fermionic elementary particles of the Standard Model . . . . . . . . . . . . . 4

2.1 Composition of one unit of the $\mathrm{NuTeV}$ calorimeter. . . . . . . . . . . . . 35

2.2 Number of neutrino and antineutrino events for each trigger . . . . . . . . . 50

2.3 Particle composition of a hadron test beam. . . . . . . . . . . . . . . . . . . 62

2.4 Parameters of the muon energy resolution function. . . . . . . . . . . . . . . 70

3.1 Number of events in each data sample. . . . . . . . . . . . . . . 75

3.2 Fit results for $\mathrm{NuTeV}$ cross section model. . . . . . . . . . . . . . . . . . 87

4.1 Non-Singlet QCD fit results with statistical errors only. . . . . . . . . . . 116

4.2 Non-Singlet QCD fit results with all systematic errors. . . . . . . . . . . . 116

4.3 Combined QCD fit results; only statistical errors. . . . . . . . . . . . . . 119

4.4 Combined QCD fit results; all systematic errors included. . . . . . . . . . . . 120

4.5 Non-Singlet QCD fit results showing the effect of each systematic. . . . . . . 123

4.6 Combined QCD fit results showing the effect of each systematic. . . . . . . . 124

4.7 Factorization scale dependence for $\overline{M S}$ model. . . . . . . . . . . . . . . . . 126

4.8 Charm mass dependence using ACOT model . . . . . . . . . . . . . . . . . 127

4.9 Bottom mass dependence for ACOT and $\overline{M S}$ models . . . . . . . . . . . . . 128

4.10 Fit results for $\overline{M S}$ model . . . . . . . . . . . . . . . . . . . . . . . . . . 131

4.11 Fit results for ACOT model . . . . . . . . . . . . . . . . . . . 132

4.12 Kinematic $x$ cut dependence. . . . . . . . . . . . . . . . . . . 133

4.13 Kinematic $Q^{2}$ dependence. . . . . . . . . . . . . . . . 133

4.14 Artificial increase of the TMC . . . . . . . . . . . . . . . . . 134 
4.15 Target mass effects on $\Lambda_{Q C D} \ldots \ldots \ldots \ldots \ldots \ldots \ldots$

B1 Data Table for Non-singlet Fit . . . . . . . . . . . . . . . . . . . . 144

B2 Data table for Combined $\mathrm{Fi} \ldots \ldots \ldots \ldots$

C1 Summary of the QCD program. . . . . . . . . . . . . . . . 149

F1 The correlation matrix for non-singlet fit. ACOT model. . . . . . . . . . . 183

F2 The correlation matrix for non-singlet fit. $\overline{M S}$ model. . . . . . . . . . . . . . 184

F3 The correlation matrix for combined fit. ACOT model. . . . . . . . . . . . . 184

F4 The correlation matrix for combined fit. $\overline{M S}$ model. . . . . . . . . . . . . . 185

G1 Test of minimization using CCFR data . . . . . . . . . . . . . . 188

G2 Testing the fitter using CCFR data . . . . . . . . . . . . . 188

G3 Fit results using CCFR data. . . . . . . . . . . . . . . . . . . . . . 189

G4 Comparison between CCFR and NuteV data. . . . . . . . . . . . . 190

G5 Fake Data study using ACOT scheme. . . . . . . . . . . . . . . . 191

G6 Comparison between $\overline{M S}$ models . . . . . . . . . . . . . . . . . . 192 


\section{LIST OF FIGURES}

1.1 Kinematics of deep inelastic lepton-nucleon scattering. . . . . . . . . . . 5

1.2 Deep Inelastic Charged Neutrino-Proton Scattering. . . . . . . . . . . . . . . 6

1.3 Helicity configurations for $\nu$ and $\bar{\nu}$ scattering off quarks and antiquarks. . . . 11

1.4 Feynman diagrams for LO and NLO DIS . . . . . . . . . . . . . 15

1.5 Feynman diagrams for processes used to measure $\alpha_{S}$. . . . . . . . . . . . 17

1.6 Running of the strong coupling $\alpha_{S} \ldots \ldots \ldots \ldots$

1.7 A sketch of the factorization theorem for hadronic structure functions. . . . . 21

1.8 Schematic representation of ACOT scheme at NLO . . . . . . . . . . . . 25

1.9 Leading Order splitting functions diagrams. . . . . . . . . . . . . . . . . 27

1.10 Examples of Next to Leading Order splitting functions diagrams. . . . . . . . 27

1.11 The size of the target mass correction for $F_{2}$ and $F_{3} \ldots \ldots \ldots$

1.12 Examples of Higher-Twist QCD diagrams. . . . . . . . . . . . . . . . 31

1.13 The size of nuclear effects for charged lepton data . . . . . . . . . . . . . 32

$2.1 \mathrm{NuTeV}$ detector . . . . . . . . . . . . . . . . . . . . . 34

2.2 A sketch of one target unit. . . . . . . . . . . . . . . 36

2.3 An illustration of a NuTeV scintillation counter. . . . . . . . . . . . . . . 37

2.4 An illustration of $\mathrm{NuTeV}$ target drift chamber section. . . . . . . . . . . . . . 39

2.5 Cross section view of a NuTeV toroid magnet with four coils. . . . . . . . . . 40

2.6 Side view of the $\mathrm{NuTeV}$ spectrometer . . . . . . . . . . . . . . . . . . 41

2.7 The effect of the burnt-out coil on the magnetic field of the toroid spectrometer. 42

2.8 Schematic view of the Fermilab Neutrino beamline . . . . . . . . . . . . 43

2.9 Structure of the beam timing . . . . . . . . . . . . . . . . . . . . 44 
2.10 A sketch of NuTeV'Sign Selected Beam . . . . . . . . . . . . . . . 45

2.11 Flux prediction of neutrino beam . . . . . . . . . . . . . . . . . 46

2.12 Readout electronics of one scintillator counter . . . . . . . . . . . . . . 48

2.13 Distribution of muon energy deposition in one scintillation counter. . . . . . 52

2.14 Average counter response to muons as function of the position. . . . . . . . . 54

$2.15 \mathrm{~A} \nu_{\mu}$ event display for a typical charged current neutrino interaction. . . . . . 55

2.16 Pulse height of an event (in MIPs) versus counter number. . . . . . . . . . . 56

2.17 A schematic view of the NuTeV Calibration Beam. . . . . . . . . . . . . 60

2.18 Particle identification among $\pi, K, \bar{p}$ at $50 \mathrm{GeV}$ hadron beam. . . . . . . . . 61

2.19 Poisson fits to ratio of hadron calorimeter energy to test beam momentum. . 63

2.20 Ratio of hadron energy response over the calibration beam momentum . . . . 64

2.21 Measured hadron resolution from fit to the width of Poisson-like distributions. 66

2.22 Poisson fits to ratio of electron calorimeter energy to test beam momentum. . 67

2.23 Location of the fitted mean $P^{T B} / P^{t o r}$ position at the front face of the toroid. 68

2.24 Detector response to muon calibration beam of $100 \mathrm{GeV}$. . . . . . . . . . . 70

3.1 A diagram of the cross-section extraction mechanism. . . . . . . . . . . . 72

3.2 Higher Twist effect on $F_{2}\left(x, Q^{2}\right)$ proton and deuteron data. . . . . . . . . 82

3.3 Feynman diagrams for QED radiative corrections . . . . . . . . . . . . . . . 84

3.4 Data - Monte Carlo comparison (neutrino mode). . . . . . . . . . . . . . . 91

3.5 Data - Monte Carlo comparison (anitneutrino mode) . . . . . . . . . . . 92

3.6 Comparison to previous neutrino measurements at $E_{\nu}=110 \mathrm{GeV}$. . . . . 98

3.7 Comparison to previous neutrino measurements at $E_{\nu}=190 \mathrm{GeV} \quad \ldots$. . . . 99

4.1 A Flow-chart of the NLO QCD fitting code. . . . . . . . . . . . . 106

$4.2 x F_{3}$ non-singlet fit using $A C O T$ and $\overline{M S}$ models. . . . . . . . . . . . . . 118

$4.3 \quad F_{2}$ from combined fit using $A C O T$ and $\overline{M S} \ldots \ldots \ldots$. . . . . . . . 121

$4.4 x F_{3}$ from combined fit using $A C O T$ and $\overline{M S} \ldots \ldots \ldots$. . . . . . . 122

4.5 Compare $\mathrm{NuTeV} \alpha_{S}\left(M_{Z^{0}}\right)$ to other experimental measurements. . . . . . . 136

D1 Control file . . . . . . . . . . . . . . . . . . . . . . . . . 151

D2 Main Program; pages 1-2. . . . . . . . . . . . . . . . 152

D3 Main Program; pages 3-4. . . . . . . . . . . . . . . . . . . 153 
D4 Main Program; pages 5-6. . . . . . . . . . . . . . . . 154

D5 Main Program; pages 7-8. . . . . . . . . . . . . . . 155

D6 Minimization Program; pages 1-2. . . . . . . . . . . . . 156

D7 Minimization Program; pages 3-4. . . . . . . . . . . . . . . 157

D8 Minimization Program; pages 5-6. . . . . . . . . . . . . 158

D9 Theory $\overline{M S}$ Program; pages 1-2. . . . . . . . . . . . . . . . . 159

D10 Theory $\overline{M S}$ Program; pages $3-4 \ldots \ldots \ldots$. . . . . . . . . . . . . 160

D11 Theory $\overline{M S}$ Program; pages $5-6 \ldots \ldots \ldots \ldots \ldots 1 . \ldots \ldots$

D12 Alpha_S Program. . . . . . . . . . . . . . . . . . . . . . . 162

D13 Evolution Code; pages 1-2. . . . . . . . . . . . . . . . 163

D14 Evolution Code; pages 3-4. . . . . . . . . . . . . . . 164

D15 Evolution Code; pages 5-6. . . . . . . . . . . . . . . . 165

D16 Evolution Code; pages 7-8. . . . . . . . . . . . . . . . 166

D17 Evolution Code; page 9-10. . . . . . . . . . . . . . . 167

E1 Constructing Structure functions using ACOT scheme . . . . . . . . . . . . . 169

E2 Program which selects ACOT scheme; pages 1-2. . . . . . . . . . . 170

E3 Program which selects ACOT scheme;pages 3-4 . . . . . . . . . . . 171

E4 ACOT program which calls the parametrized PDFs. . . . . . . . . . . . 172

E5 ACOT program calling $\alpha_{S} \ldots \ldots \ldots \ldots \ldots \ldots \ldots \ldots$

E6 Program which computes each diagram; pages 1-4. . . . . . . . . . . . 174

E7 Program which computes each diagram; pages 5-8. . . . . . . . . . . 175

E8 Program which computes each diagram; pages 9-12. . . . . . . . . . . 176

E9 Program which computes each diagram; pages 13-16. . . . . . . . . 177

E10 Program which computes each diagram; pages 17-20. . . . . . . . . 178

E11 Program which computes each diagram; pages 21-24. . . . . . . . . . . . 179

E12 Program which computes each diagram; pages 25-28. . . . . . . . . 180

E13 Program which computes each diagram; pages 29-32. . . . . . . . . . . . 181

E14 Program which computes each diagram; page 33. . . . . . . . . . . . . 182

F1 Nuclear PDFs at $Q_{0}^{2}=5 \mathrm{GeV}^{2}$. ACOT scheme. . . . . . . . . . . 186

F2 Nuclear PDFs at $Q_{0}^{2}=5 \mathrm{GeV}^{2} . \overline{M S}$ scheme. . . . . . . . . . . . . . . 186 
G1 Decomposition of the hadronic structure function. . . . . . . . . . . . . . 192

G2 Full ACOT scheme with $m_{c} \neq 0 \ldots \ldots$. . . . . . . . . . . . 193

G3 Shortcut ACOT scheme. . . . . . . . . . . . . . . . 194 


\section{PREFACE}

I here would like to thank the people who made this thesis possible. I have the pleasure to thank Donna Naples, my thesis advisor and also my friend who witnessed to my growth during these $\mathrm{PhD}$ years. She always encouraged me and provided me support. She taught me how to stand up for myself, and exposed me to several conferences that allowed me to meet extraordinary people in the field.

I am honored for being given the chance of working with Jeff Owens and Fred Olness. I would like to address my special thanks to both of them. This thesis would not have been possible without their remarkable patience and prompt guidance in this complex QCD analysis.

I thank to Jeff McDonald for helping me getting started in a field which was new to me. I am particularly grateful to him for learning various high energy physics computer skills. Many thanks to Steve Boyd, although he might enjoy the darkness, he surely shed a lot of light into the $\mathrm{NuTeV}$ analysis. Also, I would like to thank Martin Tzanov who convinced me to join the group and with whom I worked closely on the NuTeV analysis.

I would like to thank the NuTeV collaboration for their monthly feedback. Especially, I thank to Bob Bernstein, Mike Shaevitz, Randy Johnson, Arie Bodek, and Un-Ki Yang for various physics discussions and ideas. Many thanks to Dave Mason who always brought some humor during the collaboration meetings, and moreover strongly encouraged me to contact Fred Olness about the QCD code.

I would like to thank my committee members for careful reading and for suggestions in improving the manuscript. Many thanks to my friend Ted Rogers for going through the thesis and correcting my English.

I am grateful to all my friends who touched my life one way or another giving me strength 
needed in this lengthy road of $\mathrm{PhD}$.

I could not end this preface without addressing a warm thank you to my parents for inspiring me to aspire, and to Lorenzo for his moral support and belief in everything I do. 


\subsection{INTRODUCTION}

Neutrino-scattering experiments have strongly influenced the development of particle physics. The purely weak nature of neutrino interactions makes them a unique probe of nucleon structure. The focus of this thesis is to determine the coupling strength from the scale dependence of nucleon structure functions. This is a first measurement which takes quark masses into account. The measurement was performed at a low to moderate scale where there are only a few high precision measurements. It is a sensitive test of predictions of the fundamental theory of strong interactions, Quantum Chromodynamics (QCD).

In this Chapter the formalism for neutrino deep inelastic scattering (DIS) and the theory of strong interactions is discussed. This thesis is organized as follows:

- Chapter 2 provides a description of the $\mathrm{NuTeV}$ detector and neutrino beamline. The calibration of the detector and event reconstruction is also described here.

- Chapter 3 focuses on the method of extracting the differential cross sections, the structure functions, and the neutrino flux. This Chapter also presents a discussion of the systematic errors.

- Chapter 4 presents the QCD fits used to determine $\Lambda_{Q C D}$ with error analysis, and compares the results with existing measurements.

- Chapter 5 concludes and discusses future prospects for DIS measurements. 


\subsection{STANDARD MODEL AND ELEMENTARY PARTICLES}

Particle physics aims to describe the fundamental particles of the universe and their interactions. The Standard Model, developed in the 1970's, is a triumph of particle physics describing concisely the electromagnetic, weak, and strong interactions, as well as explaining the large variety of experimental observations.

In the Standard Model, the elementary particles are grouped into two categories of gauge bosons and fermions. Bosons are the integer spin particles which act as carriers of forces. Fundamental bosons - gluons, $Z^{0}, W^{ \pm}$, and photons - mediate the strong, weak, and electromagnetic interactions, respectively. Table 1.1 summarizes these particles and their interactions.

Fermions are half-integer spin particles that obey Pauli's exclusion principle and are the primary constituents of matter. They are divided further into two groups depending on which interactions they participate in: quarks and leptons.

Quarks are the only fundamental particles to experience the strong force. They also couple via all of the other fundamental interactions because they carry all of the possible charges: color, electric and weak isospin. Quarks have never been observed in free isolated states, but alway in bound states. For example, baryons represent the bound state of three quarks, and mesons are quark-antiquark pairs. Baryons and mesons are collectively named hadrons.

Leptons participate in weak interactions, and if they carry electric charge, also in electromagnetic interactions. They can exist and be observed as free particles.

Quarks and Leptons can be classified into three generations of doublets. To complete the picture of the Standard Model, each particle is associated with an anti-particle having the same mass and carrying the same charge, but of opposite sign. Fundamental fermions of the Standard Model are listed in Table 1.2 divided in three generation by successively increasing mass (from the top row down to the bottom row of the table). All the visible matter is made of the first generation particles, i.e. protons and neutrons are made of $u$ and $d$ quarks. Each generation of leptons consists of a charged and neutral lepton. The electron is the only stable particle among the charged leptons. Neutral leptons are called neutrinos 
Table 1.1: Fundamental forces described in the Standard Model with relative strengths evaluated for $Q=1 \mathrm{GeV}$

\begin{tabular}{|c|c|c|c|}
\hline \hline Force & Relative strength & Participating particles & Mediating boson \\
\hline \hline Strong & 1 & quarks & gluon \\
\hline Electromagnetic & $1.4 \times 10^{-2}$ & quarks and charged leptons & photon \\
\hline Weak & $2.2 \times 10^{-6}$ & all & $Z^{0}, W^{ \pm}$ \\
\hline \hline
\end{tabular}

and have very small mass. Quarks occur in six varieties of flavors: up, down, strange, charm, bottom, top. They carry fractional electric charge.

\subsection{DEEP INELASTIC SCATTERING AND QUARK PARTON MODEL}

The first convincing evidence for the existence of point-like constituents in the nucleon came from deep inelastic scattering (DIS) experiments performed at SLAC in the 1970's [1]. DIS is the term for the scattering of a lepton off constituents of a nucleon producing a lepton and a shower of hadrons in the final state. The scattering is called "deep" because high energy particles probe deep within the proton, and "inelastic" because the target is changed, i.e. the proton is disrupted. The kinematics of a lepton-nucleon DIS experiment is sketched in Figure 1.1.

\subsubsection{Kinematics of $\nu-N$ Deep Inelastic Scattering}

The interaction of a neutrino with a nucleon occurs via exchange of an electrically charged boson $W^{ \pm}$(charged-current interaction) or an electrically neutral boson $Z^{0}$ (neutral-current interaction). A muon-neutrino charged-current interaction has a distinctive signature that 
Table 1.2: Fermionic elementary particles of the Standard Model

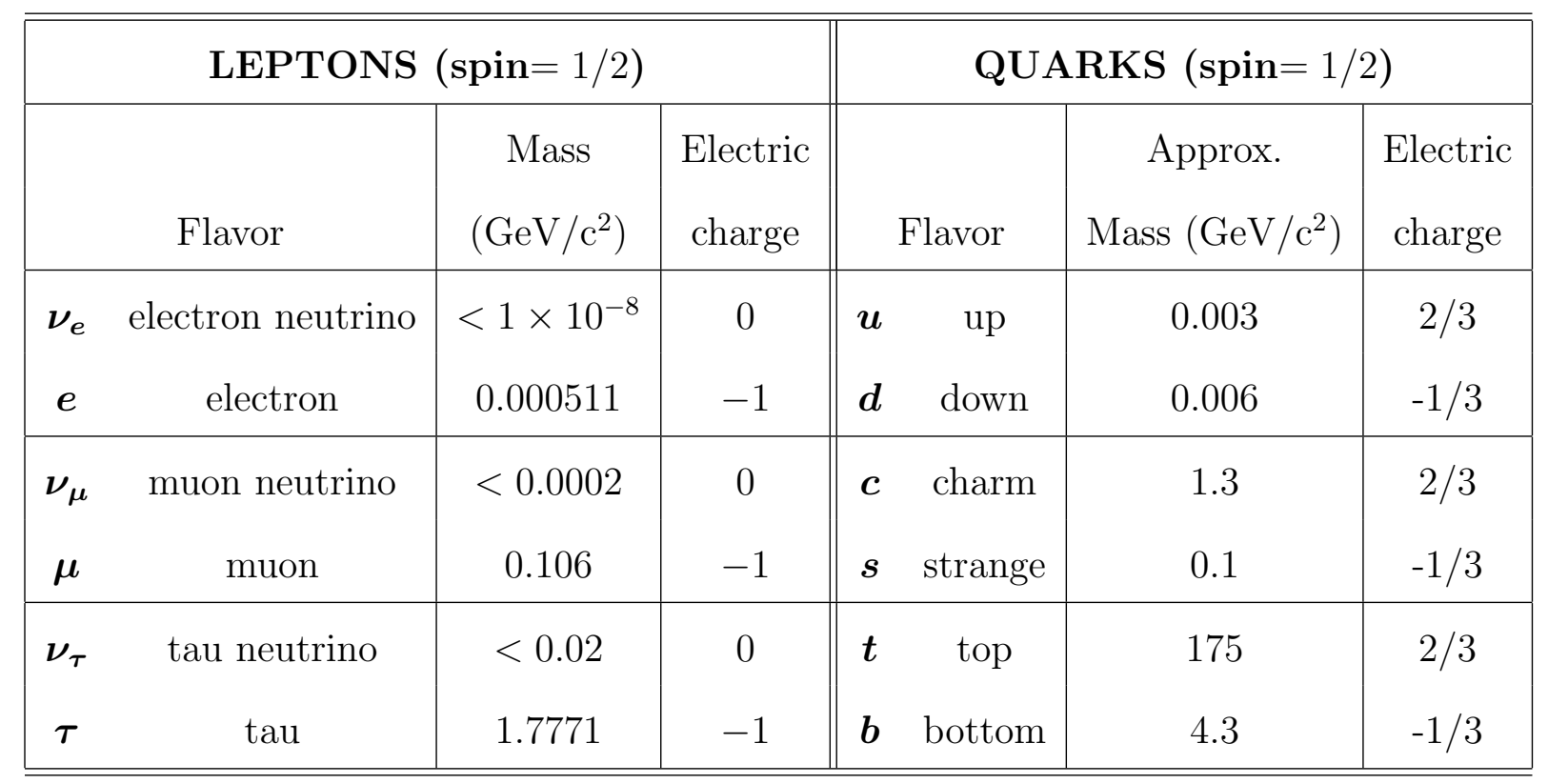

produces its associated charged lepton in the final state,

$$
\nu_{\mu}\left(\bar{\nu}_{\mu}\right)+N \rightarrow \mu^{-}\left(\mu^{+}\right)+X
$$

where $N$ is the nucleon and $X$ denotes the outgoing hadrons.

Figure 1.2 shows the scattering of an incoming high-energy neutrino with four-momentum $k$, off a hadron target with four-momentum $P$, and mass $M$. In the process, a charged $W$ boson with four-momentum $q$ is exchanged. An outgoing muon with four-momentum $k^{\prime}$ and a hadronic shower are produced in the final state. The measured quantities in the laboratory frame are the muon momentum $p_{\mu}$, the angle $\theta_{\mu}$ of the outgoing muon, and the energy $E_{\text {had }}$ of the outgoing shower of hadrons. The kinematic quantities of DIS are measured in the laboratory frame in terms of experimental quantities as

$$
\begin{aligned}
k & =\left(E_{\nu}, 0,0, E_{\nu}\right) \\
k^{\prime} & =\left(E_{\mu}, p_{\mu} \sin \theta_{\mu} \cos \phi_{\mu}, p_{\mu} \sin \theta_{\mu} \sin \phi_{\mu}, p_{\mu} \cos \theta_{\mu}\right), \\
P & =(M, 0,0,0)
\end{aligned}
$$

We want to express these quantities in terms of the following Lorentz-invariant variables: 


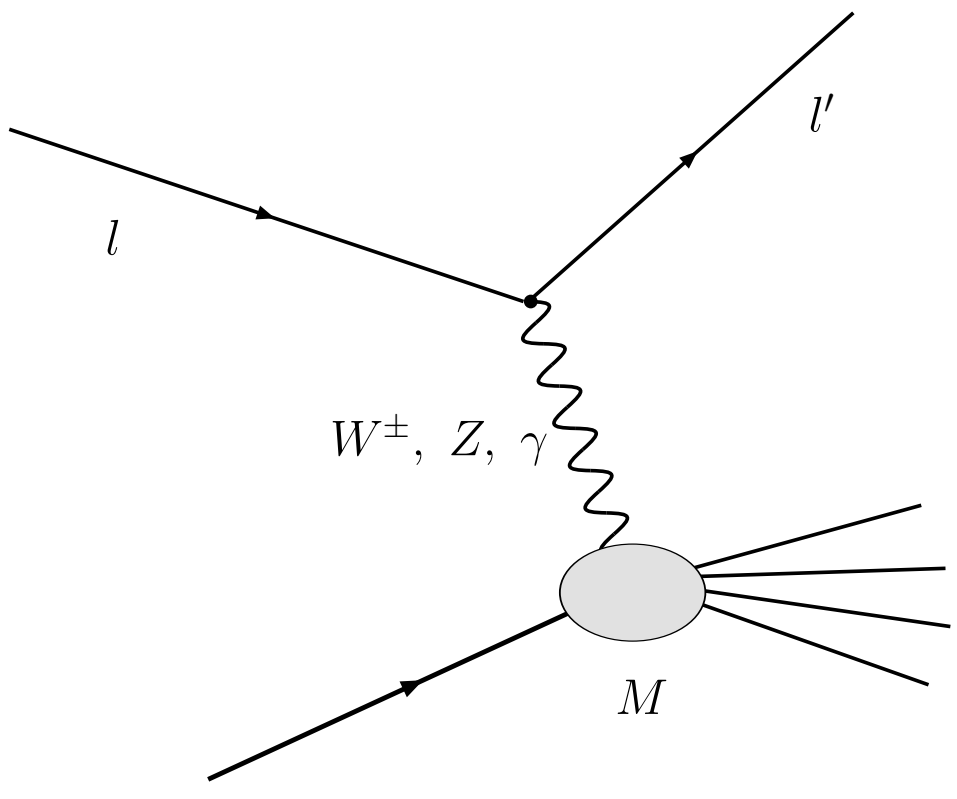

Figure 1.1: Deep inelastic scattering of a lepton off the quark constituents of a nucleon producing a lepton and a shower of hadrons in the final state.

- $Q^{2}$, the negative four-momentum squared of the exchanged boson that defines the energy scale of the interaction

$$
Q^{2}=-q^{2}=2\left(E_{\mu} E_{\nu}-\vec{k} \cdot \vec{k}^{\prime}\right)-m_{\mu}^{2}
$$

- $\boldsymbol{\nu}$, the energy transfered from the leptonic to the hadronic system

$$
\nu=\frac{q \cdot P}{M}=E_{\nu}-E_{\mu}=E_{h a d}-M
$$

- inelasticity $\boldsymbol{y}$, the fraction of the lepton's energy lost in the nucleon rest frame

$$
y=\frac{q \cdot P}{k \cdot P}=\frac{E_{h a d}}{E_{\nu}}
$$

which is related to the lepton scattering angle $\theta^{*}$ in the center-of-mass frame,

$$
y \approx 1-\frac{1}{2}\left(1+\cos \theta^{*}\right)
$$






Figure 1.2: Deep Inelastic Charged Neutrino-Proton Scattering.

- Bjorken scaling variable $\boldsymbol{x}$, the fractional momentum of the struck parton inside the nucleon $[2]$

$$
x=\frac{-q^{2}}{2 P \cdot q}=\frac{Q^{2}}{2 M \nu}
$$

- $\boldsymbol{W}^{\mathbf{2}}$, the invariant mass of the hadronic system

$$
W^{2}=(P+q)^{2}=M^{2}+2 M \nu-Q^{2}
$$

- $s$, the center-of-mass energy squared of the lepton-nucleon system

$$
s=(k+P)^{2}=\frac{Q^{2}}{x y}+M^{2} .
$$


The process in Figure 1.2 is called deep $\left(Q^{2} \gg M^{2}\right)$ inelastic $\left(W^{2} \gg M^{2}\right)$ scattering.

The cross section can be written using Fermi's golden rule

$$
d \sigma=\frac{1}{f l u x} \times|\mathcal{M}|^{2} \times d \Gamma
$$

where $\mathcal{M}$ is the invariant scattering amplitude, $d \Gamma$ is the phase space of the outgoing particles and flux is the incident-particle flux. The matrix element $\mathcal{M}$ can be expressed in terms of the leptonic and hadronic currents:

$$
\mathcal{M}=\frac{G_{F}}{\sqrt{2}} \frac{1}{1+\frac{Q^{2}}{M_{W}^{2}}} \underbrace{\bar{u}_{\mu} \gamma_{\alpha}\left(1-\gamma_{5}\right) u_{\nu}}_{\text {leptonic }} \underbrace{<X\left|J_{h}^{\alpha}\right| h>}_{\text {hadronic }}
$$

where $G_{F}$ is the weak coupling constant, $M_{W}$ is the mass of the $W$ boson, and $\bar{u}_{\mu}, u_{\nu}$ are the lepton spinors. The $\gamma_{\alpha}\left(1-\gamma_{5}\right) u_{\nu}$ is the $\mathrm{V}$-A ("vector minus axial vector") interaction, where $\gamma_{5}$ is one of the Dirac gamma matrices:

$$
\begin{aligned}
& \gamma^{0}=\left(\begin{array}{cc}
1 & 0 \\
0 & -1
\end{array}\right) \\
& \gamma^{i}=\left(\begin{array}{cc}
0 & \sigma^{i} \\
-\sigma^{i} & 0
\end{array}\right), i=1, . ., 3 \\
& \gamma^{5}=i \gamma^{0} \gamma^{1} \gamma^{2} \gamma^{3}=\left(\begin{array}{cc}
0 & 1 \\
1 & 0
\end{array}\right)
\end{aligned}
$$

and $\sigma^{i}$ are the Pauli spin matrices. The differential cross section can be factorized into leptonic $L_{\alpha \beta}$ and hadronic $H^{\alpha \beta}$ components as

$$
\frac{d^{2} \sigma_{C C}^{\nu N}}{d x d y} \propto L_{\alpha \beta} H^{\alpha \beta}
$$

The leptonic tensor is given by

$$
L_{\alpha \beta}=\left[\bar{u}(k) \gamma_{\alpha}\left(1-\gamma_{5}\right) u\left(k^{\prime}\right)\right]\left[\bar{u}\left(k^{\prime}\right) \gamma_{\beta}\left(1-\gamma_{5}\right) u(k)\right]
$$


A general form for the hadronic tensor can be constructed from the available four-vectors $\left(p^{\mu}\right.$ and $\left.q^{\mu}\right)$ and the isotropic tensors $\left(g^{\alpha \beta}\right.$ and $\left.\epsilon^{\alpha \beta \gamma \delta}\right)$ :

$$
\begin{aligned}
H^{\alpha \beta} & =-g^{\alpha \beta} W_{1}+\frac{p^{\alpha} p^{\beta}}{M^{2}} W_{2}-\frac{i \epsilon^{\alpha \beta \gamma \delta} p_{\gamma} q_{\delta}}{2 M^{2}} W_{3}+ \\
& +\frac{q^{\alpha} q^{\beta}}{M^{2}} W_{4}+\frac{p^{\alpha} q^{\beta}+p^{\beta} q^{\alpha}}{M^{2}} W_{5}+i \frac{p^{\alpha} q^{\beta}-p^{\beta} q^{\alpha}}{2 M^{2}} W_{6} .
\end{aligned}
$$

The $W_{i}$ are called structure functions which depend only on scalars $q^{2}$ and $p \cdot q$.

The time reversal invariance of QCD implies that $W_{6}=0$. The hadronic current is conserved, $\partial_{\nu} J_{h}^{\nu}=0$, hence

$$
q_{\mu} \cdot H^{\mu \nu}=0 \text { and } H^{\mu \nu} \cdot q_{\nu}=0
$$

As a consequence,

$$
\begin{aligned}
& W_{5}=-\frac{p \cdot q}{q^{2}} W_{2}, \\
& W_{4}=\left(\frac{p \cdot q}{q^{2}}\right)^{2} W_{2}+\frac{M^{2}}{q^{2}} W_{1} .
\end{aligned}
$$

Evaluating the contraction of leptonic and hadronic tensors, the $W_{4}$ and the $W_{5}$ terms are proportional to the lepton mass and can be neglected because they are small relative to other scales. This leaves us with three remaining structure functions $W_{1}, W_{2}$ and $W_{3}$. The latter is the parity-violating structure function, accessible only by neutrino scattering.

In deep inelastic scattering, the nucleon is resolvable for $Q, \nu \gg M$, and the structure functions depend on the Lorentz scalar variables $\nu$ and $Q^{2}$. In the DIS regime $(Q, \nu \gg M)$, large $Q^{2}$ resolves point-like constituents inside the proton. In the $Q^{2}, \nu \rightarrow \infty$ limit, the functions $W_{i}\left(Q^{2}, \nu\right)$ depend (approximately) only on the ratio $x=Q^{2} / 2 M \nu$. This feature of structure functions is known as Bjorken scaling [2]. However, experimental observations show small $Q^{2}$ dependences of the structure functions (scaling violations).

The dimensionless structure functions are defined as follows:

$$
\begin{aligned}
2 x F_{1} & \equiv 2 x M W_{1}, \\
F_{2} & \equiv \nu W_{2}, \\
x F_{3} & \equiv \nu x W_{3} .
\end{aligned}
$$


Thus, the general form for differential neutrino-nucleon DIS cross-section can be written in terms of three independent structure functions $2 x F_{1}\left(x, Q^{2}\right), F_{2}\left(x, Q^{2}\right)$ and $x F_{3}\left(x, Q^{2}\right)$ as

$$
\frac{d^{2} \sigma^{\nu(\bar{\nu}) N}}{d x d y}=\frac{G_{F}^{2} M E_{\nu}}{\pi\left(1+Q^{2} / M_{W}^{2}\right)^{2}}\left[\frac{y^{2}}{2} 2 x F_{1}^{\nu(\bar{\nu}) N}+\left(1-y-\frac{M x y}{2 E_{\nu}}\right) F_{2}^{\nu(\bar{\nu}) N} \pm y\left(1-\frac{y}{2}\right) x F_{3}^{\nu(\bar{\nu}) N}\right]
$$

The differential cross section is often expressed in terms of the longitudinal structure function $R_{L}\left(x, Q^{2}\right)$ defined as the ratio of the cross-sections for longitudinally $\left(\sigma_{L}\right)$ polarized to transversely $\left(\sigma_{T}\right)$ polarized $W$ bosons. The interpretation of $R_{L}$ can be inferred by expressing the structure functions in terms of cross-sections for longitudinally $\left(\sigma_{L}\right)$ and transversely $\left(\sigma_{T}\right)$ polarized $W$ boson:

$$
\begin{aligned}
& F_{1}=\frac{M K}{\pi G_{F} \sqrt{2}} \sigma_{T}, \\
& F_{2}=\frac{\nu K}{\pi G_{F} \sqrt{2}} \frac{Q^{2}}{Q^{2}+\nu^{2}}\left(\sigma_{T}+\sigma_{L}\right),
\end{aligned}
$$

where $K$ denotes the flux of virtual bosons, and $\nu=Q^{2} / 2 M x$. Thus, $R_{L}$ relates $F_{2}$ to $2 x F_{1}$ as

$$
R_{L}=\frac{\sigma_{L}}{\sigma_{T}}=\frac{F_{2}\left(x, Q^{2}\right)\left(1+4 M^{2} x^{2} / Q^{2}\right)-2 x F_{1}\left(x, Q^{2}\right)}{2 x F_{1}\left(x, Q^{2}\right)}
$$

In this analysis we will use the neutrino-nucleon differential cross-section expressed in terms of $R_{L}, F_{2}$, and $x F_{3}$ as

$$
\frac{d^{2} \sigma^{\nu(\bar{\nu}) N}}{d x d y}=\frac{G_{F}^{2} M E_{\nu}}{\pi\left(1+Q^{2} / M_{W}^{2}\right)^{2}}\left[\left(\frac{y^{2}+\frac{4 M^{2} x^{2} y^{2}}{Q^{2}}}{2+2 R_{L}^{\nu(\bar{\nu}) N}}+1-y-\frac{M x y}{2 E_{\nu}}\right) F_{2}^{\nu(\bar{\nu}) N} \pm y\left(1-\frac{y}{2}\right) x F_{3}^{\nu(\bar{\nu}) N}\right]
$$




\subsubsection{Structure Functions in The Quark Parton Model}

Early evidence for Bjorken scaling in electron DIS scattering experiments [1] led to the formulation of the parton model (Bjorken and Pashos, 1969; Feynman, 1972). In this model, the hadron is viewed as an assembly of non-interacting point-like particles named partons, which are interpreted as quarks and gluons. This model is valid in the "infinite momentum frame" in which the characteristic time scale for the strong interactions among partons inside the nucleon is much longer than the collision time. In this frame, the neutrino-nucleon scattering can be viewed as elastic scattering between neutrinos and point-like partons inside the nucleon which carry a fraction $x$ of nucleon's momentum.

Structure functions are described in terms of momentum-weighted parton distribution functions (PDFs) which describe the probability that the interacting parton carries a fraction $x$ of the hadron's longitudinal momentum.

Elastic scattering of two point particles depends on the center-of-mass energy and the net angular momentum of the system. Neutrinos and anti-neutrinos are spin- $\frac{1}{2}$ fermions, hence the total angular momentum from the scattering of two spin- $\frac{1}{2}$ particles may be either 0 or \pm 1 . The $\mathrm{V}$-A nature of the weak charged current operator selects only left-handed particles and right-handed anti-particles. Therefore, there are only four possible helicity configurations for scattering of two spin- $\frac{1}{2}$ particles as illustrated in Figure 1.3. Neutrinos are left-handed (helicity $=-1$ ), hence their spin is aligned in the opposite direction as their momentum, while antineutrinos are right-handed (helicity $=+1$ ) with their spin aligned in the same direction as their momentum. As a result of angular momentum conservation, the spin-zero contributions to the total charged current cross sections are given by $\nu q(\overline{\nu q})$ interactions, while the spin-one contributions are given by $\nu \bar{q}(\bar{\nu} q)$ interactions. As shown in Figure 1.3, the spin-zero configuration has no angular dependence (i.e. no $y$ dependence), while the spin-one has $(1-y)^{2}$ dependence.

From the above helicity arguments, the neutrino differential cross sections for elastic 




Figure 1.3: Possible helicity configurations for neutrinos and anti-neutrinos scattering from quarks and antiquarks. The top rows depict the configurations for $\vec{J}_{z}=0$, without preferred scattering direction. The bottom rows shows the configurations for $\vec{J}_{z}= \pm 1$.

scattering of neutrinos with spin- $\frac{1}{2}$ partons can be derived as

$$
\begin{aligned}
& \frac{d^{2} \sigma^{\nu}}{d x d y}=\frac{2 G_{F}^{2} M E}{\pi}\left[x q(x)+(1-y)^{2} x \bar{q}(x)\right], \\
& \frac{d^{2} \sigma^{\bar{\nu}}}{d x d y}=\frac{2 G_{F}^{2} M E}{\pi}\left[x \bar{q}(x)+(1-y)^{2} x q(x)\right] .
\end{aligned}
$$

We can relate neutrino structure functions to the parton densities from Eqs. (1.27), (1.32) and (1.33),

$$
\begin{aligned}
2 x F_{1}^{\nu(\bar{\nu})}\left(x, Q^{2}\right) & =2\left[x q^{\nu(\bar{\nu})}(x)+x \bar{q}^{\nu(\bar{\nu})}(x)\right], \\
x F_{3}^{\nu(\bar{\nu})}\left(x, Q^{2}\right) & =2\left[x q^{\nu(\bar{\nu})}(x)-x \bar{q}^{\nu(\bar{\nu})}(x)\right] .
\end{aligned}
$$

For purely spin- $\frac{1}{2}$ constituents, the Callan-Gross relation [3] holds,

$$
2 x F_{1}=F_{2},
$$

as confirmed by early experiments at SLAC [1].

Neutrino charged current scattering resolves the flavor of nucleon quarks due to the charge conservation at the quark vertex. Thus, neutrinos can scatter only from $d, s, \bar{u}, \bar{c}$ 
quarks, while antineutrinos scatter only from $\bar{d}, \bar{s}, u, c$ quarks. For a proton target, the quark densities contributing to the structure functions are

$$
\begin{array}{ll}
q^{\nu p}(x)=d^{p}(x)+s^{p}(x), & q^{\bar{\nu} p}(x)=u^{p}(x)+c^{p}(x), \\
\bar{q}^{\nu p}(x)=\bar{u}^{p}(x)+\bar{c}^{p}(x), & \bar{q}^{\bar{\nu} p}(x)=\bar{d}^{p}(x)+\bar{s}^{p}(x) .
\end{array}
$$

For a neutron target, the quark densities are

$$
\begin{array}{ll}
q^{\nu n}(x)=d^{n}(x)+s^{n}(x), & q^{\bar{\nu} n}(x)=u^{n}(x)+c^{n}(x), \\
\bar{q}^{\nu n}(x)=\bar{u}^{n}(x)+\bar{c}^{n}(x), & \bar{q}^{\bar{\nu} n}(x)=\bar{d}^{n}(x)+\bar{s}^{n}(x) .
\end{array}
$$

The quark content of a proton and a neutron is related through isospin symmetry

$$
\begin{aligned}
u^{p}(x)=d^{n}(x) \equiv u(x), & \bar{u}^{p}(x)=\bar{d}^{n}(x) \equiv \bar{u}(x), \\
d^{p}(x)=u^{n}(x) \equiv d(x), & \bar{d}^{p}(x)=\bar{u}^{n}(x) \equiv \bar{d}(x), \\
s^{p}(x)=s^{n}(x) \equiv s(x), & \bar{s}^{p}(x)=\bar{s}^{n}(x) \equiv \bar{s}(x), \\
c^{p}(x)=c^{n}(x) \equiv c(x), & \bar{c}^{p}(x)=\bar{c}^{n}(x) \equiv \bar{c}(x) .
\end{aligned}
$$

Therefore, for an isoscalar target, which has equal number of protons and neutrons, we have

$$
\begin{aligned}
q^{\nu N}(x)=\frac{1}{2}[u(x)+d(x)+2 s(x)], & q^{\bar{\nu} N}(x)=\frac{1}{2}[u(x)+d(x)+2 c(x)], \\
\bar{q}^{\nu N}(x)=\frac{1}{2}[\bar{u}(x)+\bar{d}(x)+2 \bar{c}(x)], & \bar{q}^{\bar{\nu} N}(x)=\frac{1}{2}[\bar{u}(x)+\bar{d}(x)+2 \bar{s}(x)] .
\end{aligned}
$$

The neutrino-nucleon structure functions $2 x F_{1}^{\nu N}$ and $x F_{3}^{\nu N}$ for an isoscalar target are obtained by using Eqs. (1.34), (1.35),

$$
\begin{aligned}
2 x F_{1}^{\nu N} & =x u(x)+x d(x)+2 x s(x)+x \bar{u}(x)+x \bar{d}(x)+2 x \bar{c}(x), \\
x F_{3}^{\nu N} & =x u(x)+x d(x)+2 x s(x)-x \bar{u}(x)-x \bar{d}(x)-2 x \bar{c}(x) .
\end{aligned}
$$

For antineutrinos, only the positively charged quarks contribute

$$
\begin{aligned}
2 x F_{1}^{\bar{\nu} N} & =x u(x)+x d(x)+2 x s(x)+x \bar{u}(x)+x \bar{d}(x)+2 x \bar{c}(x), \\
x F_{3}^{\bar{\nu} N} & =x u(x)+x d(x)-2 x s(x)-x \bar{u}(x)-x \bar{d}(x)+2 x \bar{c}(x) .
\end{aligned}
$$


The quarks can be separated into valence quarks [66], which carry charge and sea quarks and antiquarks which carry no net charges. The quark and antiquark distributions are given by

$$
\begin{aligned}
x q(x) & =x q_{v}(x)+x q_{s}(x), \\
x \bar{q}(x) & =x \bar{q}_{s}(x), \\
x q_{s}(x) & =x \bar{q}_{s}(x),
\end{aligned}
$$

where $q_{v}$ denotes the valence quark distribution, $q_{s}$ is the sea quark distribution, and $\bar{q}$ the antiquark distribution. To characterize structure functions, we note that $2 x F_{1}^{\nu(\bar{\nu}) N}$ is related to the sum of quark and antiquark distributions in the nucleon. The average $x F_{3}^{a v e}$ structure function,

$$
x F_{3}^{a v e}=\frac{x F_{3}^{\nu N}+x F_{3}^{\bar{\nu} N}}{2}=x u_{v}+x d_{v}
$$

is sensitive to valence-quark distributions. The difference $\Delta x F_{3}$,

$$
\Delta x F_{3}=x F_{3}^{\nu N}-x F_{3}^{\bar{\nu} N}=4(x s-x c)
$$

is sensitive to the strange and charm distributions.

For charged-leptons the scattering is mediated by virtual photons. In this case the structure function $x F_{3}$ is not present. The electromagnetic charged-lepton structure functions are sensitive to quark charges $e_{i}$,

$$
2 x F_{1}^{l N}=\sum_{i} e_{i}^{2}\left(x q_{i}^{l}+x \bar{q}_{i}^{l}\right)
$$




\subsection{QUANTUM CHROMODYNAMICS}

The quark parton model does not consider dynamics between quarks. The theory that describes strong interactions among colored quarks and gluons is called Quantum Chromodynamics (QCD) [Fritzsch et al., 1973; Gross and Wilczek, 1973; Weinberg 1973]. The theory of QCD was born as a result of assembling many ideas and experimental results. In terms of the group symmetries, QCD is the $S U(3)$ component of the $S U(3) \times S U(2) \times U(1)$ Standard Model of the fundamental particles.

The QCD Lagrangian density that describes the interactions of quarks and gluons is given, up to gauge-fixing terms, by

$$
\begin{aligned}
\mathrm{L}_{Q C D} & =-\frac{1}{4} F_{\mu \nu}^{(a)} F^{(a) \mu \nu}+i \sum_{q} \overline{\psi_{q}^{i}} \gamma^{u}\left(D_{\mu}\right)_{i j} \psi_{q}^{j} \\
& -\sum_{q} m_{q} \overline{\psi_{q}^{i}} \psi_{q i} \\
F_{\mu \nu}^{(a)} & =\partial_{\mu} A_{\nu}^{a}-\partial_{\nu} A_{\mu}^{a}-g_{s} f_{a b c} A_{\mu}^{b} A_{\nu}^{c}, \\
\left(D_{\mu}\right)_{i j} & =\delta_{i j} \partial_{\mu}+i g_{s} \sum_{a} \frac{\lambda_{i, j}^{a}}{2} A_{\mu}^{a},
\end{aligned}
$$

where $\psi_{q}^{i}(x)$ are the four-component Dirac spinors associated with each quark field of color $i,(i=1, . ., 3)$, and flavor $q, A_{\mu}^{a}(x)$ are gluon fields (of which there are eight), $g_{s}$ is the QCD coupling constant, and $f_{a b c}$ are the structure constants of the $\mathrm{SU}(3)$ algebra.

Quarks have never been observed free. The confinement of quarks is related to the strength of the long distances interaction. The key feature of the theory of QCD is asymptotic freedom, (Gross and Wilczek, 1973; Politzer 1973), which is related to the weakness of the short distances processes.

\subsubsection{Perturbative QCD and $\alpha_{S}$}

In perturbative QCD (pQCD), calculations are performed by expanding terms in a perturbation series in the coupling strength $\alpha_{S}$. This is only valid when $\alpha_{S}$ is small (which happens at high $Q^{2}$, as shown in Figure 1.6 and discussed in Section 1.3.1.2). The calculation of a scattering cross section in perturbative QCD reduces to summing over the amplitudes of 
all possible intermediate states. Each graph is a symbolic representation for a term in the perturbative calculation. Leading Order (LO) term corresponds to the Quark Parton Model and is considered to be of order $\alpha_{S}^{0}$ in the perturbative expansion. LO Feynman diagrams have no gluon vertices as shown in Figure 1.4 (A). Next-to-Leading Order (NLO) diagrams add quark-gluon interactions to this pictures. NLO graphs have one gluon vertex and correspond to terms of order $\alpha_{S}^{1}$ in the perturbative expansion. NLO Feynman diagrams for hard scattering are illustrated in Figure 1.4 (B). Following this idea, higher order terms (NNLO, NNNLO, etc) would correspond to addition of more gluon vertices (two, three, etc).



(A)


$(B)$

Figure 1.4: (A) Feynman diagrams for LO DIS process, and (B) NLO DIS process with gluon or quark initiated processes.

\subsubsection{Renormalization}

The four-momentum is conserved at each vertex. However, including higher order diagrams 
(i.e. loop diagrams), the momentum circulating in the loop is not constrained. The integration over all momentum space for a loop diagram leads to logarithmic divergences when momentum goes to infinity, as

$$
\int d^{4} k \frac{1}{k^{2}\left[(k+p)^{2}-m^{2}\right]} \sim \int d \Omega_{3} \int d k k^{3} \frac{1}{k^{2} \cdot k^{2}} \sim \int \frac{d k}{k}
$$

This type of divergences, called ultraviolet (UV) divergences, is removed by a procedure called "renormalization". This procedure introduces an arbitrary parameter $\mu$ with dimension of mass, representing the point at which the subtraction of the UV divergence is performed.

\subsubsection{Running of $\alpha_{S}$}

Any physical observable $\mathcal{R}$ must be independent of the choice for $\mu$, therefore we impose the following condition:

$$
\mu^{2} \frac{d R}{d \mu^{2}}=\left(\mu^{2} \frac{\partial}{\partial \mu^{2}}+\mu^{2} \frac{\partial \alpha_{s}}{\partial \mu^{2}} \frac{\partial}{\partial \alpha_{S}}\right)=0
$$

Hence, any explicit dependence of the physical observable on the renormalization scale must be canceled by a proper renormalization scale dependence of $\alpha_{S}$. The strong coupling $\alpha_{S}$ is determined by renormalization group equation given by

$$
Q^{2} \frac{\partial \alpha_{S}}{\partial Q^{2}}=\beta\left(\alpha_{S}\right)=-\alpha_{S} \sum_{n=0}^{\infty} \beta_{n}\left(\frac{\alpha_{S}}{4 \pi}\right)^{(n+1)}=-\frac{\beta_{0}}{4 \pi} \alpha_{S}^{2}\left(Q^{2}\right)-\frac{\beta_{1}}{16 \pi^{2}} \alpha_{S}^{3}+O\left(\alpha_{S}^{4}\right)
$$

where the coefficients $\beta_{0}$ and $\beta_{1}$ depend on the number of active quark flavors $n_{f}$ and scale $Q^{2}$ as

$$
\begin{aligned}
& \beta_{0}=11-\frac{2}{3} n_{f} \\
& \beta_{1}=102-\frac{38}{3} n_{f} .
\end{aligned}
$$

Expansion of the $\beta$-function is carried out to two loops, which corresponds to a NLO analysis. 
The solution to renormalization group Eq. (1.54) provides the scale dependence of the strong coupling $\alpha_{S}$, i.e. the "running" of $\alpha_{S}$. Perturbative QCD predicts the scale dependence of the strong coupling, but $\alpha_{S}$ at a specific scale is obtained from experiment. Therefore, $\alpha_{S}$ at a reference scale is a fundamental parameter of the theory of QCD. Measuring the strong coupling from various experiments at different characteristic energy scales is an important test of QCD.

There are different physics processes from where $\alpha_{S}$ can be determined, such as DIS, $e^{-} e^{+}$ annihilations, hadron collisions, or quarkonia decays. The corresponding Feynman graphs for these processes are shown in Figure 1.5. Figure 1.6 shows the experimental measurements


Figure 1.5: Feynman diagrams for processes used to measure $\alpha_{S}$. The first graph corresponds to DIS process, the second to $e^{+} e^{-}$annihilation, the third to hadron collisions, and last one to quarkonia decays

of $\alpha_{S}$ over a large range in $Q^{2}$. The decrease in $\alpha_{S}\left(Q^{2}\right)$ with increasing $Q$ (i.e. "running of $\left.\alpha_{S}{ }^{\prime \prime}\right)$ is clearly observed. The standard convention of the reference scale is chosen to be the $Z$ mass. The value of $\alpha_{S}$ at different scales can be determined from the following relation:

$$
\log \frac{Q^{2}}{M_{Z}^{2}}=\int_{\alpha_{S}\left(M_{Z}\right)}^{\alpha_{S}(Q)} \frac{d \alpha_{S}}{\beta\left(\alpha_{S}\right)}
$$

where $M_{Z}$ is the mass of the $Z$ boson. 


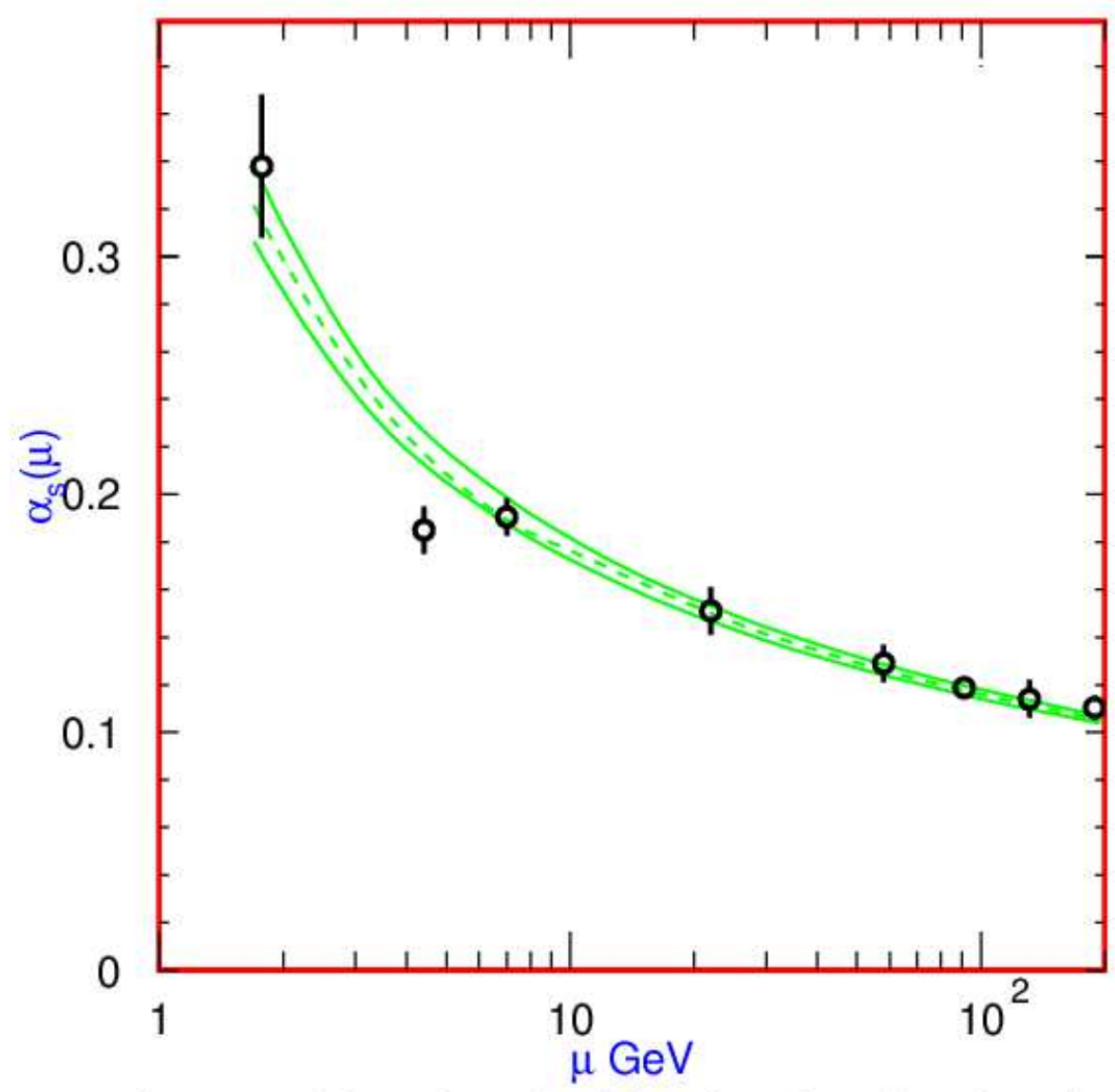

Figure 1.6: Running of the strong coupling $\alpha_{S}$ with scale $\mu \equiv Q$. The lines show the world average and the $\pm 1 \sigma$. The measurements correspond, in increasing order of $\mu$, to: $\tau$ width, $Y$ decays, DIS, $e^{+} e^{-}$event shapes at 22 and $58 \mathrm{GeV}, Z^{0}$ width and $e^{+} e^{-}$event shapes shapes at 135 and $189 \mathrm{GeV}$ (taken from PDG 2005).

\subsubsection{3 $\Lambda_{Q C D}$ parameter}

Alternatively, one can introduce a dimensional parameter, $\Lambda_{Q C D}$, to provide a parametrization of the scale dependence of the strong coupling $\alpha_{S}$. Following the convention from Ref.[4], $\Lambda_{Q C D}$ is defined by writing the solution of the renormalization group equation at a NLO as: 


$$
\alpha_{S}\left(Q^{2}\right)=\frac{4 \pi}{\beta_{0} \ln \frac{Q^{2}}{\Lambda_{Q C D}^{2}}}\left[1-\frac{\beta_{1} \ln \left(\ln \frac{Q^{2}}{\Lambda_{Q C D}^{2}}\right)}{\beta_{0}^{2} \ln \frac{Q^{2}}{\Lambda_{Q C D}^{2}}}\right] .
$$

$\Lambda_{Q C D}$ represents the scale at which perturbative QCD becomes strongly coupled, i.e. the scale for which the coupling $\alpha_{S}$ is large and the perturbative QCD theory breaks down. This scale is comparable with the masses of the light hadrons $(\approx 0.5 \mathrm{GeV})$. In other words, $\Lambda_{Q C D}$ determines the boundary between quasi-free state of interacting quarks and gluons (weak coupling) and the state where hadrons are formed (strong coupling). In this analysis we determine $\Lambda_{Q C D}$ from the NLO fits to the scaling violations of the nucleon structure functions, which is then related to the strong coupling $\alpha_{S}$ through Eq. (1.58).

\subsubsection{Quark Thresholds}

When quark mass effects are taken into account, $n_{f}$ becomes a discontinuous function of the scale: below the charm threshold, three active flavor quarks are considered, while above, $n_{f}$ depends on whether the scale is above the mass threshold for heavy quarks, i.e. $n_{f}=4$ for scale between the charm and bottom threshold, $n_{f}=5$ above the bottom quark threshold, etc.

The matching prescription is imposed by the condition that $\alpha_{S}$ must be a continuous function for all momenta, including the region of crossing a quark threshold. Thus, if Eq. (1.58) is to remain valid with $\alpha_{S}$ remaining continuous in $Q^{2}$, but $n_{f}$ discontinuous in $Q^{2}$, then the effective value of $\Lambda_{Q C D}$ will depend on the number of active quark flavors. The matching conditions are given by

$$
\begin{aligned}
& \alpha_{S}^{n_{f}=4}\left(m_{b}^{2}\right)=\alpha_{S}^{n_{f}=5}\left(m_{b}^{2}\right), \\
& \alpha_{S}^{n_{f}=3}\left(m_{c}^{2}\right)=\alpha_{S}^{n_{f}=4}\left(m_{c}^{2}\right) .
\end{aligned}
$$

For the scale range probed by the fixed-target DIS experiments, it is most appropriate to quote $\Lambda_{Q C D}$ for four active quark flavors. One can make modifications to account for 
fewer or greater number of active flavors using Eqs. (1.58-1.56). Also, $\Lambda_{Q C D}$ depends on the renormalization scheme and on the order to which the calculation is carried. Therefore, when quoting a value for $\Lambda_{Q C D}$ one must specify the associated $n_{f}$, order and renormalization scheme explicitly.

\subsubsection{Factorization}

Perturbative calculations are performed in context of the factorization theorem which states that structure functions can be written in a factorized form with a soft (non-perturbative) and a hard (calculable, process-dependent) component [5],

$$
F^{W N}=\sum_{i} f_{N}^{a} \otimes \omega^{W a}+O\left(\frac{\Lambda^{2}}{Q^{2}}\right)
$$

with the sum carried over all interacting quarks and gluons. The symbol $\otimes$ stands for the convolution between the parton distribution functions, $f_{N}^{a}$, and the hard scattering coefficients, $\omega^{W a}$. It is defined as

$$
g \otimes h=\int_{x}^{1} \frac{d y}{y} g(u) h\left(\frac{x}{y}\right) .
$$

Figure 1.7 illustrates a handbag diagram of the factorization theorem. The factorization scale, $\mu_{F}$, defines the separation between soft (non-calculable) and hard (calculable using Feynman rules, ultraviolet dominated) physics. For processes with scales $\mu_{F}>Q$, the physics is non-perturbative QCD and contained in the $\mathrm{PDF}, f_{N}^{a}$, while for $\mu_{F}<Q$ the physics is perturbative QCD (calculable) contained in the hard scattering coefficients $\omega^{W a}$. 




Figure 1.7: Illustration of the factorization theorem for hadronic structure functions. Process on the left $N(P)+W(q) \rightarrow X(P x)$ and the factorized process on the right $N(P) \rightarrow a\left(k_{1}\right)$ (represented by PDF, $f_{N}^{a}$ ) with the successive hard scattering interaction $a\left(k_{1}\right)+W(q)$ (represented by $\left.\omega^{a}\right)$.

\subsubsection{QCD Theory Models}

There are various approaches of dividing the structure functions into calculable processes and PDFs. This analysis is presenting two NLO QCD models used in determination of $\alpha_{S}$. The $\overline{M S}$ scheme provides a massless calculation of the structure functions and the ACOT scheme fully accounts for quark masses. These models are described separately in the following subsections.

\subsubsection{Massless $\overline{M S}$ Scheme}

This is a model that deals with two scales: $\Lambda_{Q C D}$, which describes the scale of a hadronic bound-state, and $Q^{2}$, the defining scale of large virtuality. This calculation is carried out under the assumption of massless quarks. The $\overline{M S}$ factorization scheme uses dimensional regularization to remove UV divergences [6]. The structure functions are obtained to NLO from the convolution of the hard scattering coefficients (process dependent) with the parametrized 
PDFs (process independent). The quark distributions are conveniently divide into nonsinglet, $q^{N S}$, and singlet, $q^{S}$, quark distributions:

$$
\begin{aligned}
q^{N S} & =\sum_{i}\left(q_{i}-\bar{q}_{i}\right)=u_{v}+d_{v} \\
q^{S} & =\sum_{i}\left(q_{i}+\bar{q}_{i}\right) .
\end{aligned}
$$

The structure functions at NLO can be written as follows:

$$
F_{3}\left(x, Q^{2}\right)=\int_{x}^{1} \frac{d y}{y}\left[C_{3}^{q}\left(\frac{x}{y}, Q^{2}\right) q^{N S}\left(x, Q^{2}\right)\right]
$$

$$
\frac{F_{2}\left(x, Q^{2}\right)}{x}=\int_{x}^{1} \frac{d y}{y}\left[C_{2}^{q}\left(\frac{x}{y}, Q^{2}\right) q^{S}\left(x, Q^{2}\right)+C_{2}^{g}\left(\frac{x}{y}, Q^{2}\right) G\left(x, Q^{2}\right)\right]
$$

where the $C_{i}$ are the coefficient functions which depend on the factorization and renormalization schemes. The $\overline{M S}$ coefficient functions are calculated at NLO in Ref. [7].

To leading order $\left(\alpha_{S}^{0}\right)$ the coefficients of quark terms are proportional to $\delta\left(1-\frac{x}{y}\right)$, while the coefficients of gluon induced terms vanish. Therefore, the structure functions in the LO can be written simply as,

$$
\begin{gathered}
x F_{3}\left(x, Q^{2}\right)=x q^{N S}\left(x, Q^{2}\right), \\
F_{2}\left(x, Q^{2}\right)=x q^{S}\left(x, Q^{2}\right) .
\end{gathered}
$$




\subsubsection{ACOT Scheme}

In heavy quark production, the presence of the heavy quark mass, $M_{H}$, introduces a third scale. The heavy quarks will require a different organization of the perturbation series for an efficient separation of the short-distance and long-distance parts depending on the relative magnitude of $M_{H}$ and the characteristic energy scale of the process. The factorization theorem has been extended by Collins [8] to incorporate quark masses as,

$$
F=\sum_{a} \int \frac{d y}{y} f_{N}^{a}\left(x, \mu_{F}\right) \otimes \omega_{h / a}\left(\frac{x}{y}, \frac{Q}{\mu_{F}}, \frac{M_{H}}{\mu_{F}}, \alpha_{S}\left(\mu_{R}\right)\right)+O\left(\frac{\Lambda_{Q C D}}{Q}\right),
$$

where $\omega_{h / a}$ represents the coefficient functions and $f_{N}^{a}$ is the parton density. The index $a$ is over all quark flavors. There are different approaches to the treatment of heavy quark production. These include the Fixed Flavor Number (FFN) and Variable Flavor Number (VFN) schemes [9]. Both schemes are equivalent if calculations are carried out to all orders in $\alpha_{S}$.

\section{- FFN scheme}

Heavy quark contributions are explicitly included in the hard cross sections. This is intended for the factorization scale $\mu_{F}$ in the range of $M_{H}$ (for $M_{H} \sim \mu_{F}$ ). In this case, heavy-quark production can be calculated from hard processes initiated by light quarks and gluons, and effects of the charm mass are included in the perturbative coefficient functions. This procedure gives problems when $\mu_{F} \gg M_{H}$ because the coefficient functions at higher order in $\alpha_{S}$ contain large logarithms that spoil the perturbative series $[10]$.

\section{- VFN scheme}

PDFs for heavy quarks are introduced and the number of active flavors changes by one unit when a heavy quark threshold is crossed. In other words, when $M_{H} \gg \mu_{F}$ this heavy quark should be excluded from the hadron (decoupling) and when $M_{H} \ll \mu_{F}$ it should be included. In this scheme the large logarithms which were encountered in the FFN 
scheme are re-summed via heavy quark PDFs, hence the convergence of the perturbative series is preserved [11].

The Aivazis-Collins-Olness-Tung $(A C O T)$ scheme [12] belongs to the group of VFN factorization schemes that uses the renormalization method of Collins-Wilczek-Zee (CWZ) [8]. This scheme involves a mixture of the $\overline{M S}$ scheme for light partons (and for heavy partons if $\mu_{F}>M_{H}$ ) and the zero-momentum subtraction renormalization scheme for graphs with heavy quark lines (if $\mu_{F}<M_{H}$ ). The NLO ACOT scheme is graphically presented in Figure 1.8. The first graph corresponds to the heavy quark excitation subprocess, $W q \rightarrow q$ (order $\alpha_{S}^{0}$ quark-scattering); the second to the heavy quark creation subprocess, $W g \rightarrow q q$ (order $\alpha_{S}^{1}$ gluon-fusion); and the third to the subtraction term.

The $A C O T$ scheme is designed to deal with finite quark masses, but neutrino structure functions have both light and heavy quark contributions,

$$
F_{i}^{\nu}\left(x, Q^{2}\right)=F_{i, l}^{\nu}\left(x, Q^{2}\right)+F_{i, c}^{\nu}\left(x, Q^{2}\right)
$$

where $l$ stands for light quark flavor, and $c$ for charm quark flavor. Therefore, this analysis uses a variant of the ACOT scheme which still fully accounts for heavy quark production, but is numerically more stable when dealing with light quarks because the light quark masses are set to zero. The results in the limit $M_{H} \rightarrow 0$ agree with the massless $\overline{M S}$ case, as it should (see Appendix G.3.3 for more details).

\subsubsection{Evolution and QCD Sum Rules}

The $Q^{2}$ dependence of quark momentum densities is described by the coupled, integrodifferential Dokshitzer-Gribov-Lipatov-Altarelli-Parisi (DGLAP) evolution equations [13]. For the non-singlet case the evolution doesn't depend on the gluon distribution, while the 



Figure 1.8: Schematic representation of ACOT scheme at NLO.

singlet distribution co-evolves with the gluon distribution, as can be seen from the following relations:

$$
\begin{aligned}
\frac{\partial q^{N S}}{\partial \ln Q^{2}} & =\frac{\alpha_{S}\left(Q^{2}\right)}{2 \pi} P_{q q} \otimes q^{N S} ; \\
\frac{\partial}{\partial \ln Q^{2}}\left(\begin{array}{c}
q^{S} \\
g
\end{array}\right) & =\frac{\alpha_{S}\left(Q^{2}\right)}{2 \pi}\left(\begin{array}{cc}
P_{q q} & 2 n_{f} P_{q g} \\
P_{g q} & P_{g g}
\end{array}\right) \otimes\left(\begin{array}{c}
q^{S} \\
g
\end{array}\right),
\end{aligned}
$$

where the symbol $\otimes$ denotes the convolution operator. These evolution equations can be expressed in terms of

$$
t=\ln \frac{Q^{2}}{\Lambda^{2}} ; \quad s=\ln \frac{\ln \frac{Q^{2}}{\Lambda^{2}}}{\ln \frac{Q_{0}^{2}}{\Lambda^{2}}}=\ln \frac{t}{t_{0}}
$$

as

$$
\begin{gathered}
\frac{d q^{N S}(x, s)}{d s}=t \frac{\alpha_{S}(t)}{2 \pi} \int_{x}^{1} \frac{d y}{y} q^{N S}(y, s) P_{q q}^{N S}\left(\frac{x}{y}\right) \\
\frac{d q^{S}(x, s)}{d s}=t \frac{\alpha_{S}(s)}{2 \pi} \int_{x}^{1} \frac{d y}{y}\left(q^{S}(y, s) P_{q q}^{S}\left(\frac{x}{y}\right)+G(y, s) P_{q G}\left(\frac{x}{y}\right)\right) \\
\frac{d G(x, s)}{d s}=t \frac{\alpha_{S}(s)}{2 \pi} \int_{x}^{1} \frac{d y}{y}\left(q^{S}(y, s) P_{G q}^{S}\left(\frac{x}{y}\right)+G(y, s) P_{G G}\left(\frac{x}{y}\right)\right)
\end{gathered}
$$


The splitting functions $P_{i j}\left(\frac{x}{y}\right)$ give the probability of parton $j$ with momentum fraction $y$ be resolved as parton $i$ with momentum fraction $x<y$. They are calculated perturbatively to a given order in $\alpha_{S}$. LO and NLO splitting function diagrams are shown in Figs.1.9, 1.10. Evolution equations describe the physical picture in which valence quarks are surrounded by a cloud of virtual particles which are continuously emitted and absorbed. The quarks are also emitting and absorbing virtual particles of their own, corresponding in these equations to the branching probability densities. This picture explains why the structure of the hadron appears to change as it is seen at different distance scales. Thus, at low $Q^{2}$ there are fewer partons and their PDFs are skewed to high momentum fractions. At high $Q^{2}$, the momentum is shared through the parton branchings, and hence the low $\mathrm{x}$ region is filled with gluons and sea quarks which have a high probability to undergo $g \rightarrow g g, g \rightarrow q \bar{q}$ branchings. QCD doesn't predict the PDFs at any scale, rather it predicts how PDFs evolve with the scale through the evolution equations.

As a remark, in this analysis a massless evolution of the PDFs has been used regardless of the above selected theory model In fact, it has been shown in Ref. [14] that in a consistently formulated perturbative QCD as long as proper matching between the hard scattering and PDFs is observed, the final result is the same whether massless or massive evolution is used.

The PDFs are constrained through the following conservation laws, referred to as QCD sum rules:

- Fermion sum rule: In the simple parton model the fermion sum rule is the integral over the valence quark distributions with an expectation of 3 for proton(neutron),

$$
\int_{0}^{1} d x u_{v}(x)=2 ; \quad \int_{0}^{1} d x d_{v}(x)=1 .
$$

Since the number of bosons is not conserved, gluon distributions do no have such constraint. 




Figure 1.9: Leading Order splitting functions diagrams.




$P_{G G}$

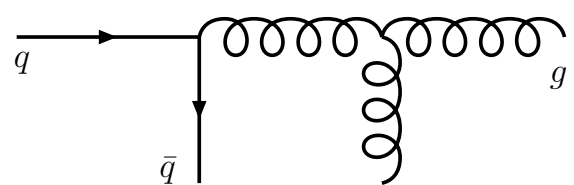

$P_{G q}$

Figure 1.10: Examples of Next to Leading Order splitting functions diagrams. 
- Momentum sum rule: The momentum sum rule states that the total momentum of the proton carried by quarks and gluons is 1 ,

$$
\int_{0}^{1}\left(x q_{S}+x G\right) d x=1 .
$$

This rule is used to constrain the gluon normalization parameter.

\subsubsection{Non-Perturbative QCD Effects}

At low $Q^{2}$, the strong coupling constant becomes large and the perturbative calculations fail. In this non-perturbative region the assumption of scattering from massless, point-like, quarks is no longer valid. Also, the resolving power is not large enough to probe a single quark scattering. To obtain high interaction rates, neutrino experiments use heavy targets. Therefore, nuclear effects must be considered as well. These non-perturbative effects are discussed in the following subsections.

\subsubsection{Target Mass}

At low $Q^{2}$ and high $x$, one can not neglect the effects of the target mass. The meaning of $x$ as the fraction of the nucleon's momentum carried by the struck quark is not suitable when $Q^{2} \approx M^{2}$. A "target mass" correction must be applied to account for these effects.

The target mass correction (TMC) to the structure functions have been first determined by Georgi and Politzer in 1976 [15]. More recently, new derivations were performed [16] which lead to the following relations: 


$$
\begin{aligned}
F_{1}^{T M}\left(x, Q^{2}\right) & =\frac{x}{\xi} \frac{F_{1}^{(0)}\left(\xi, Q^{2}\right)}{k}+\frac{M_{p}^{2} x^{2}}{Q^{2} k^{2}} \int_{\xi}^{1} \frac{F_{2}^{(0)}\left(u, Q^{2}\right)}{u^{2}} d u \\
& +\frac{2 M_{p}^{4} x^{3}}{Q^{4} k^{3}} \int_{\xi}^{1} d u \int_{u}^{1} \frac{F_{2}^{(0)}\left(v, Q^{2}\right)}{v^{2}} d v \\
F_{2}^{T M}\left(x, Q^{2}\right) & =\frac{x^{2}}{\xi^{2}} \frac{F_{2}^{(0)}\left(\xi, Q^{2}\right)}{k^{3}}+\frac{6 M_{p}^{2} x^{3}}{Q^{2} k^{4}} \int_{\xi}^{1} \frac{F_{2}^{(0)}\left(u, Q^{2}\right)}{u^{2}} d u \\
& +\frac{12 M_{p}^{4} x^{4}}{Q^{4} k^{5}} \int_{\xi}^{1} d u \int_{u}^{1} \frac{F_{2}^{(0)}\left(v, Q^{2}\right)}{v^{2}} d v \\
F_{3}^{T M}\left(x, Q^{2}\right) & =\frac{x}{\xi} \frac{F_{3}^{(0)}\left(\xi, Q^{2}\right)}{k^{2}}+\frac{2 M_{p}^{2} x^{2}}{Q^{2} k^{3}} \int_{\xi}^{1} \frac{F_{3}^{(0)}\left(u, Q^{2}\right)}{u} d u
\end{aligned}
$$

with $\xi$ and $k$ defined as

$$
k=\sqrt{1+\frac{4 x^{2} M_{p}^{2}}{Q^{2}}}, \quad \xi=\frac{2 x}{1+k} .
$$

Sometimes the longitudinal structure function, $F_{L}$ is used instead of $F_{2}$ or $F_{1}$ structure functions. $F_{L}$ is defined as the combination between the absorption of transversely $\left(F_{1}\right)$ and longitudinally $\left(F_{2}-2 x F_{1}\right)$ polarized virtual bosons as follows:

$$
F_{L}\left(x, Q^{2}\right)=\left(1+\frac{4 M^{2} x^{2}}{Q^{2}}\right) F_{2}\left(x, Q^{2}\right)-2 x F_{1}\left(x, Q^{2}\right) .
$$

One can then compute the target mass corrected longitudinal structure function:

$$
\begin{aligned}
F_{L}^{\mathrm{TMC}}\left(x, Q^{2}\right) & =k^{2} F_{2}^{\mathrm{TMC}}\left(x, Q^{2}\right)-2 x F_{1}^{\mathrm{TMC}}\left(x, Q^{2}\right) \\
& =\frac{x^{2}}{\xi^{2} k}\left[F_{2}^{(0)}(\xi)-2 \xi F_{1}^{(0)}(\xi)\right]+\frac{4 M_{p}^{2} x^{3}}{Q^{2} k^{2}} \int_{\xi}^{1} \frac{F_{2}^{(0)}\left(u, Q^{2}\right)}{u^{2}} d u \\
& +\frac{8 M_{p}^{4} x^{4}}{Q^{4} k^{3}} \int_{\xi}^{1} d u \int_{u}^{1} \frac{F_{2}^{(0)}\left(v, Q^{2}\right)}{v^{2}} d v \\
& =\frac{x^{2}}{\xi^{2} k} F_{L}^{(0)}(\xi)+\frac{4 M_{p}^{2} x^{3}}{Q^{2} k^{2}} \int_{\xi}^{1} \frac{F_{2}^{(0)}\left(u, Q^{2}\right)}{u^{2}} d u \\
& +\frac{8 M_{p}^{4} x^{4}}{Q^{4} k^{3}} \int_{\xi}^{1} d u \int_{u}^{1} \frac{F_{2}^{(0)}\left(v, Q^{2}\right)}{v^{2}} d v .
\end{aligned}
$$

Here are some clarifying remarks on these equations:

- The target mass corrected structure functions are functions of $\left(x, Q^{2}\right)$ and not $\left(\xi, Q^{2}\right)$. 
- The relations are general, holding to all orders in $\alpha_{S}$.

- Quark masses effects are taken into account in $F_{i}^{(0)}$.

- $F_{L}^{T M C}\left(x, Q^{2}\right)$ is non-zero and thus violates the Callan-Gross relation, as it should. The leading term $\left(F_{L}^{(0)}\right)$ will be non-zero for finite quark masses, and the sub-leading terms that involve integrals are non-zero for finite hadron mass $M_{p}$.

The target mass effects are large at high $x$ and low $Q^{2}$. Figure 1.11 shows the size of the the TMC for both $F_{2}\left(x, Q^{2}\right)$ and $F_{3}\left(x, Q^{2}\right)$ structure functions as function of $x$. The size of the corrections increases with decreasing scale, as shown in Figure 1.11 by various colors for $Q^{2}=4,8,16,32,64,128 \mathrm{GeV}^{2}$


Figure 1.11: The size of the target mass correction for $F_{2}$ and $F_{3}\left(\frac{T M_{O N}}{T M_{O F F}}\right)$. Red, green, blue, cyan, magenta, and yellow colors correspond to 4, 8, 16, 32, 64, and $128 \mathrm{GeV}^{2}$, respectively

\subsubsection{Higher-Twist}

The operators in pQCD are classified according to their twist, $\tau$, defined as the difference between the free field dimension $d$ and the spin $j$ of the operator. The leading operators are these of order twist-2 which do not consider inter-quarks interactions. Twist $\tau>2$ terms (higher-twists) are suppressed by increasing powers of $\left(1 / Q^{2}\right)^{n}$ with respect to the leading twist. The higher-twist terms include interactions with other quarks, as shown in 
Figure 1.12. Since target mass effects also involve powers of $1 / Q^{2}$, they are referred to as "kinematic higher-twist" effects. The scattering involving a conglomerate of quarks processes are referred to as "dynamical higher-twist". These effects are important at low $Q^{2}$ and high $x$.
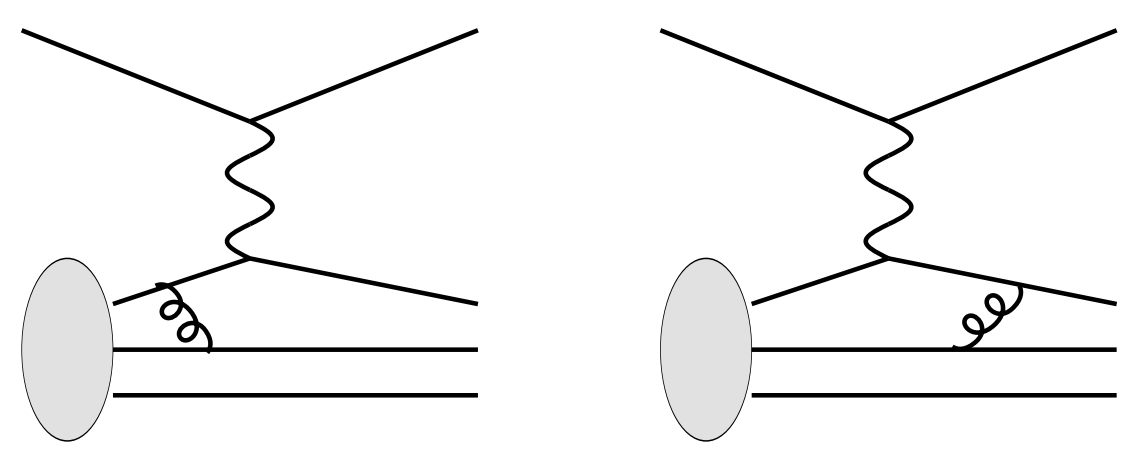

Figure 1.12: Examples of Higher-Twist QCD diagrams.

The higher-twist contributions are calculated using phenomenological approaches. However, our analysis uses data in the kinematic range where these effects are negligible. Therefore only kinematical higher-twist effects are studied (target mass effects). No additional highertwist correction is applied.

\subsubsection{Nuclear Target}

It has been observed in the charged lepton scattering experiments (SLAC [17], NMC [18], EMC [19]) that the nuclear environment in which a struck quark is embedded modifies the $x$ distribution of the quarks inside the nucleon. Figure 1.13 shows the size of nuclear target effects as function of $x$. Although limited data exists for nuclear effects on neutrino scattering, the standard correction for these effects is obtained from a fit to charged-lepton scattering on nuclear targets. This correction is large at low and high $x$, but is independent of $Q^{2}$. Hence, it does not affect the extraction of $\Lambda_{Q C D}$ from QCD fits to $Q^{2}$ dependence of structure functions.

There are new theoretical treatments that consider a $Q^{2}$ dependent nuclear target cor- 


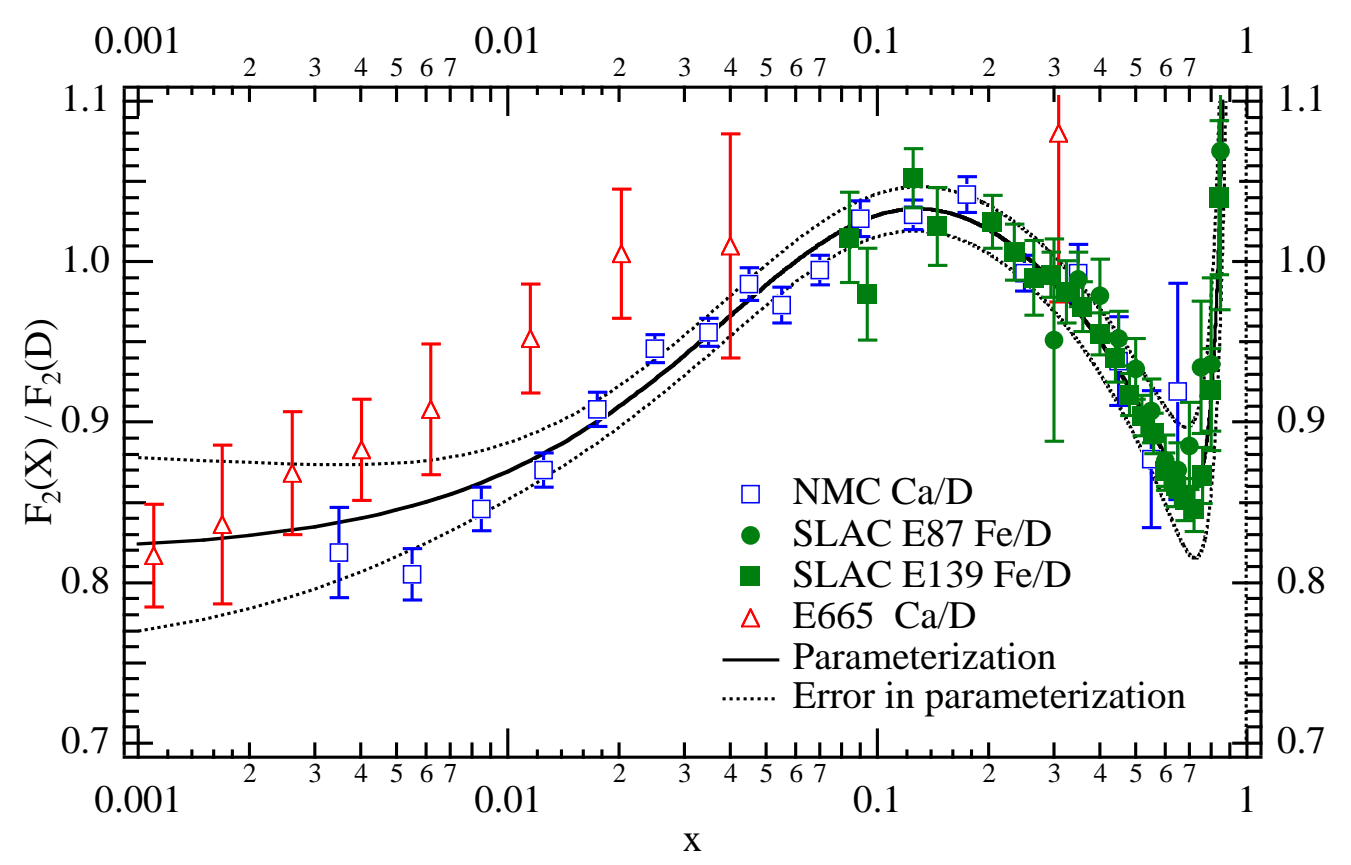

Figure 1.13: The size of nuclear effects as function of $x$ for charged lepton data from Ref. $[17,18,19]$. Plot taken from Ref. [50].

rections at low $x[20,21]$. Recent results from $\mathrm{NuTeV}$ hint that neutrino experiments might favor smaller nuclear effects than the charged lepton experiments [22] at high $x$.

This thesis does not take into account $Q^{2}$ dependent nuclear corrections. We can interpret our extracted PDFs from QCD fits as "effective nuclear PDFs", which have the nuclear effects absorbed into them. 


\subsection{NUTEV EXPERIMENT}

In the previous Chapter, we outlined the theoretical background of deep inelastic neutrino scattering. In order to examine a neutrino interaction, it is necessary to provide a neutrino beam and a target with a detector to record the events. $\mathrm{NuTeV}$ is a precision neutrino-iron DIS experiment which collected separate high statistics neutrino and antineutrino events in 1996-97 at Fermilab. The unique design of NuTeV lead to the achievement of the following physics goals:

- A precise measurement of the electro-weak mixing angle [23];

- A search for neutral heavy leptons and other new processes[24, 27];

- A search for $\nu_{\mu} \rightarrow \nu_{e}$ oscillations [25];

- A measurement of the strange quark sea [26];

- A measurement of the neutrino-iron differential cross sections [22].

The final $\mathrm{NuTeV}$ result is a measurement of the strong coupling constant from QCD scaling violations of structure functions, which is the focus of this thesis. These results depend on a detailed understanding of the response of the target-calorimeter. We present in this Chapter the experimental setup of $\mathrm{NuTeV}$ including the detector and the neutrino beam. The calibration of the detector and data reconstruction are also presented in this Chapter. 


\section{$2.1 \quad$ NUTEV DETECTOR}

Neutrinos interact only weakly. Hence, to increase the statistical precision of neutrino measurements a large dense target is often used. The NuTeV detector is composed of an iron target calorimeter followed immediately downstream by an iron toroidal muon spectrometer. This 1000 ton detector is illustrated in Figure 2.1. The two components are described in the next sections.

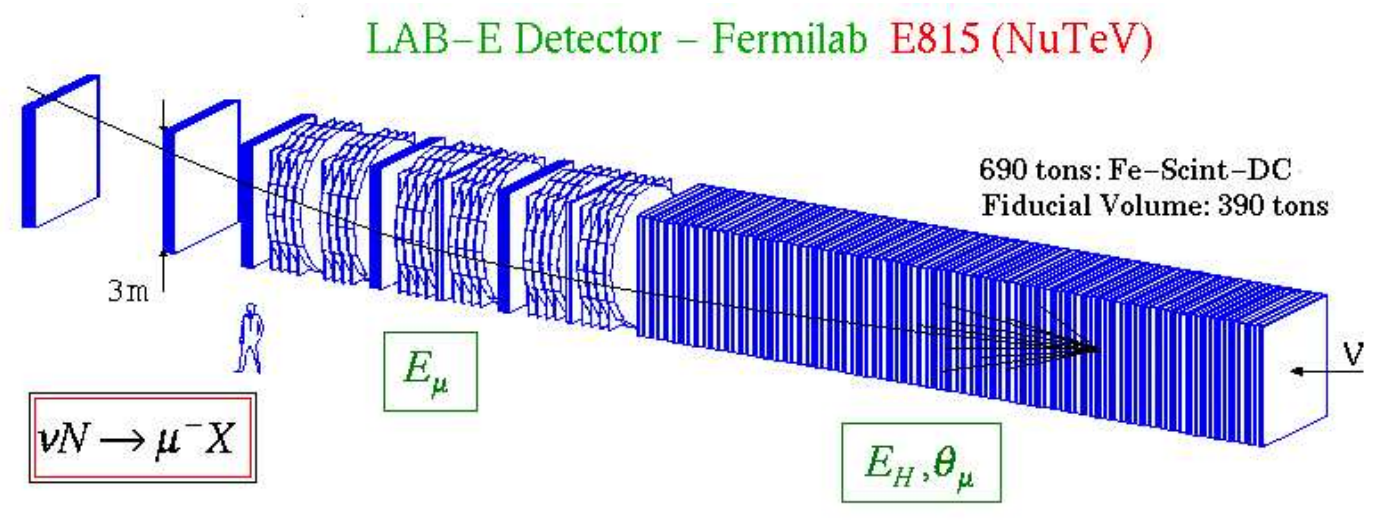

Figure 2.1: NuTeV detector

\subsubsection{Target Calorimeter}

The target calorimeter is $18 \mathrm{~m}$ long and weighs 690 ton. It is composed of a passive part of 168 plates of steel $(3 \mathrm{~m}(\mathrm{H}) \times 3 \mathrm{~m}(\mathrm{~W}) \times 5.1 \mathrm{~cm}(\mathrm{~L}))$, and an active part of 84 scintillation counters $(3 \mathrm{~m}(\mathrm{H}) \times 3 \mathrm{~m}(\mathrm{~W}) \times 2.5 \mathrm{~cm}(\mathrm{~L}))$ and 42 drift chambers. One calorimeter unit consists of two scintillation counters, each sandwiched between two steel plates, and a drift chamber placed every four steel plates, i.e. $10.35 \mathrm{~cm}$ of steel between scintillation counters and $20.7 \mathrm{~cm}$ of steel between drift chambers (see Figure 2.2). The composition of one unit of $\mathrm{NuTeV}$ target calorimeter in length, interaction length $\left(\lambda_{I}\right)$ and radiation length $\left(\lambda_{\text {rad }}\right)$ is given in Table 2.1 [28]. This unit is repeated 42 times to make up the whole calorimeter. 
Table 2.1: Composition in radiation length and interaction length of one unit of the NuTeV calorimeter.

\begin{tabular}{|l|c|c|c|}
\hline \hline Component & $\begin{array}{c}\text { Length } \\
(\mathrm{cm})\end{array}$ & $\begin{array}{c}\text { Radiation Length } \\
\lambda_{\text {rad }}\end{array}$ & $\begin{array}{c}\text { Interaction Length } \\
\lambda_{I}\end{array}$ \\
\hline \hline 4 Steel Plates & 20.7 & 11.75 & 1.24 \\
\hline 2 Scint. Counter & 13.0 & 0.51 & 0.16 \\
\hline 1 Drift Chamber & 3.7 & 0.17 & 0.03 \\
\hline \hline Total & 37.4 & 12.43 & 1.43 \\
\hline \hline
\end{tabular}

Neutrino interactions take place in the steel plates (high $Z$ material) producing hadron shower and muons sampled by scintillation counters and drift chambers (low $Z$ material).

- Scintillation counters: are used to measure the energy deposited by charged particles in the calorimeter. The 84 liquid scintillation counters are numbered starting from the most upstream counter (84) to the most downstream counter (1). Each counter is instrumented with four photomultiplier tubes (PMT) located at each corner of the counter. A schematic view of a scintillation counter is shown in Figure 2.3. One scintillation counter is a $3 \mathrm{~m} \times 3 \mathrm{~m}$ and $2.5 \mathrm{~cm}$ thick Lucite box filled with 65 gallons of Bicron $517 \mathrm{~L}$ liquid scintillator oil. Lucite ribs do not scintillate, therefore they are spaced about $2.5-5 \mathrm{~cm}$ apart and staggered to avoid formation of dead zones. Two plastic water-filled bags are positioned on each side of the counter to balance the pressure of the oil.

The mechanism of a scintillation counter is as follows: a charged current particle passing through the scintillator excites primary flours in the oil which emit photons as ultraviolet light. This light is absorbed by secondary flours in the oil, radiating visible blue light. The blue light is transmitted to the edges of the counter through total internal 


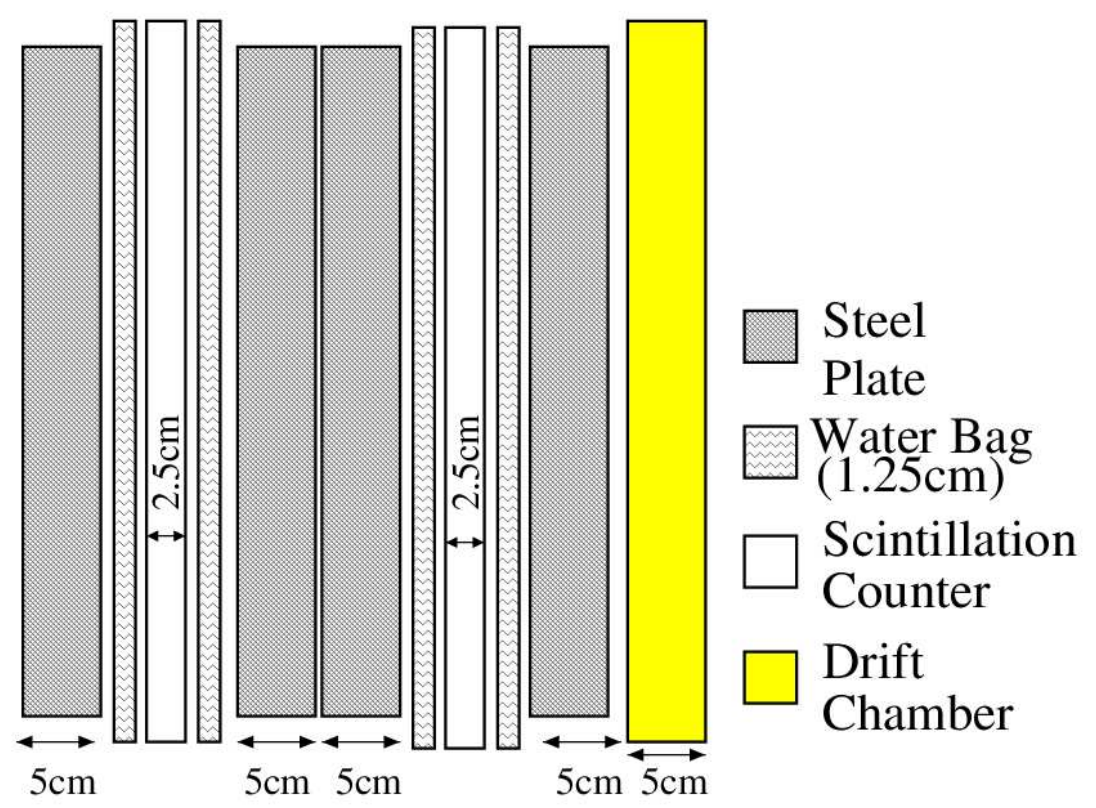

Figure 2.2: A sketch of one target unit consisted from steel plates, water bags, scintillation counters and one drift chamber

reflection where it is absorbed by eight half-inch thick wavelength-shifter bars (WLS) which shift blue light to green light of longer wavelength. This green light is piped to the phototubes mounted at the corner of each counter. The 10-stage Hamamatsu R2154 phototubes with green-extended photocathodes operate with a potential drop of $1400 \mathrm{~V}$ across the cathode to anode. The photocathode has about $20 \%$ probabibility of emitting an electron via the photoelectric effect. A single electron is amplified by a factor of $10^{6}$ by a series of dynodes. A typical signal for a muon passing through the center of the counter is about 30 photoelectrons, and higher near the edge.

- Drift chambers: are used to track the transverse positions of the muons inside the calorimeter target. Each drift chamber consists of two orthogonally oriented planes with 24 horizontal and 24 vertical cells each filled with an equal mixture of argon and ethane 

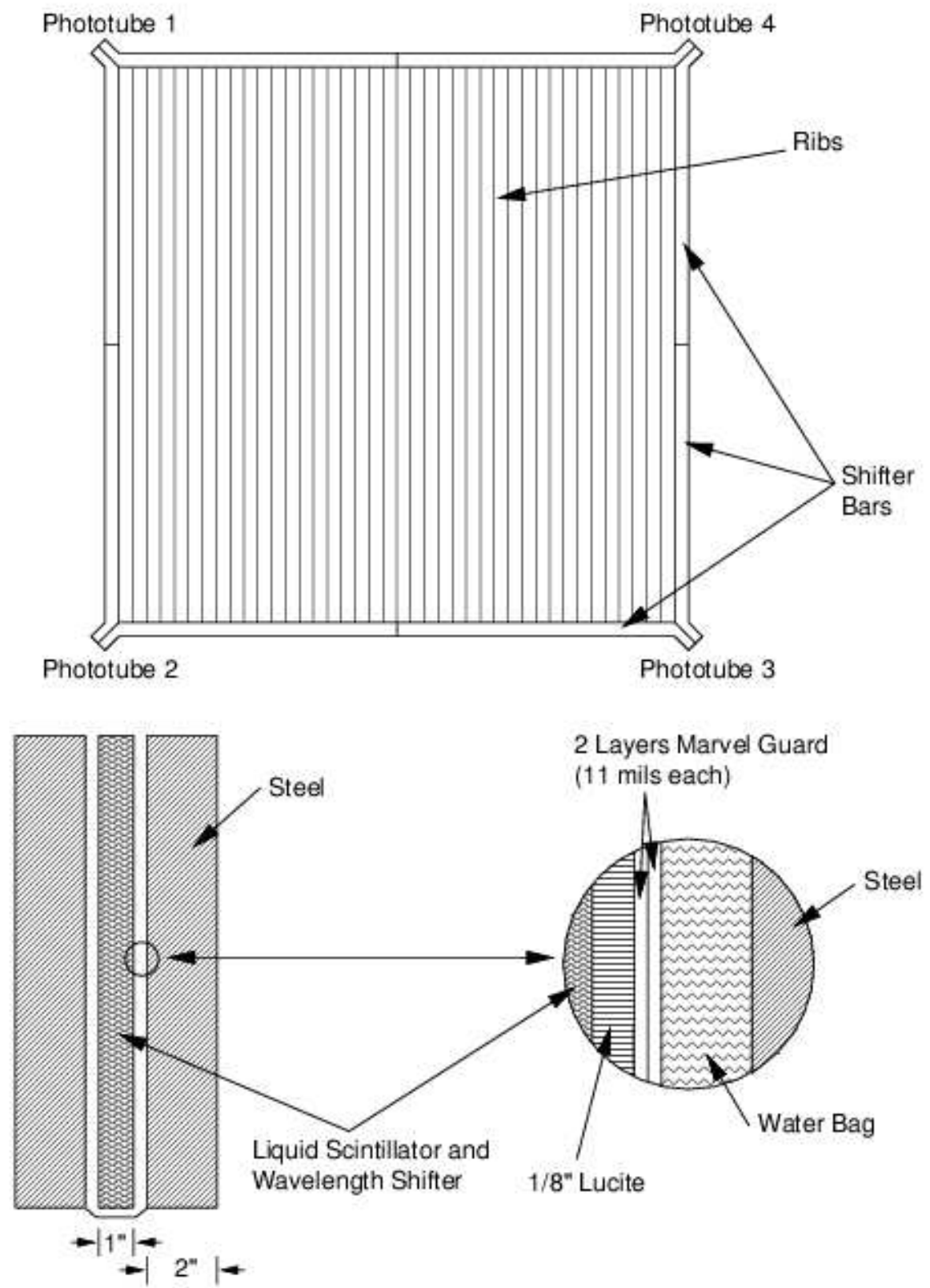

Figure 2.3: An illustration of a NuTeV scintillation counter.

gas. Each cell is 5 inches wide and contains three wires at center. The sketch of a drift chamber is shown in Figure 2.4. The drift chamber cells are defined by parallel aluminum I-beams which support G10 cover plates. For each cell the G10 cover is clad with 19 
copper cathode strips set at a voltage to maintain a uniform electric fields of $690 \mathrm{~V} / \mathrm{cm}$. The central field wire within each cell is held at $+350 \mathrm{~V}$. On each side of the field wire there are two sense wires held at $+1750 \mathrm{~V}$. The I-beams operate at $-4500 \mathrm{~V}$. Hence, for a constant drift velocity of $52.4 \mu \mathrm{m} / \mathrm{s}$ the drift time for electrons to cross 2.5 in inside a cell is $1.2 \mu \mathrm{s}$.

A charged particle passing through a chamber cell ionizes gas molecules along its path. The electrons drift in an electric field toward the anode (sense) wire and accelerate producing further ionization. A signal pulse from collected electrons is registered on the sense wire. The particle's position in one dimension is determined by comparing the time of the electron in the uniform field $\left(T^{0}\right)$ to the known time of the event $(T)$. The position in the $X-Y$ plane of the charged particle is determined by combining hit information from two paired orthogonal cells as follows:

$$
\begin{aligned}
& x=x^{0}+v_{D}\left(T-T_{x}^{0}\right), \\
& y=y^{0}+v_{D}\left(T-T_{y}^{0}\right),
\end{aligned}
$$

where $x^{0}, y^{0}$ denote the locations of sense wires, and $v_{D}$ is the drift velocity of free electrons.

\subsubsection{Muon Spectrometer}

The muon spectrometer is located immediately downstream of the target calorimeter. It consists of three large toroidal magnets. Each magnet is composed of eight steel washers with an outer radius of $1.8 \mathrm{~m}$ and an inner radius of $0.12 \mathrm{~m}$. The toroids are magnetized by four copper coils which carry a DC current of 1250 A. Figure 2.5 shows a sketch of a cross sectional view of the toroid.

To track the passing muons, magnets are instrumented with five sets of single-wire drift chamber arrays. Three sets are located in the gaps between the toroids, and the remaining 




Figure 2.4: An illustration of a NuTeV drift chamber section. There are two orthogonallyoriented planes per station, consisting of three-wire cells.

two are downstream of the last toroid (blue carts). The blue carts are used to measure the high momentum muons. Figure 2.6 shows a side view of the muon spectrometer with toroidal magnets and drift chambers.

The toroid magnetic field varies between 1.9 T near the hole and $1.5 \mathrm{~T}$ near the edge. A muon passing through the whole length of the spectrometer receives a transverse momentum kick of $2.4 \mathrm{GeV}$. The polarity of the magnetic field is set to always focus on the primary muon, i.e. for neutrino mode $\mu^{-}$are bent towards the center, for anti-neutrino mode $\mu^{+}$are focused. This enables $\mathrm{NuTeV}$ to better reconstruct muon momentum.

Early in the run, one of the coils of the second toroid (marked with asterisk in Figure 2.5) 


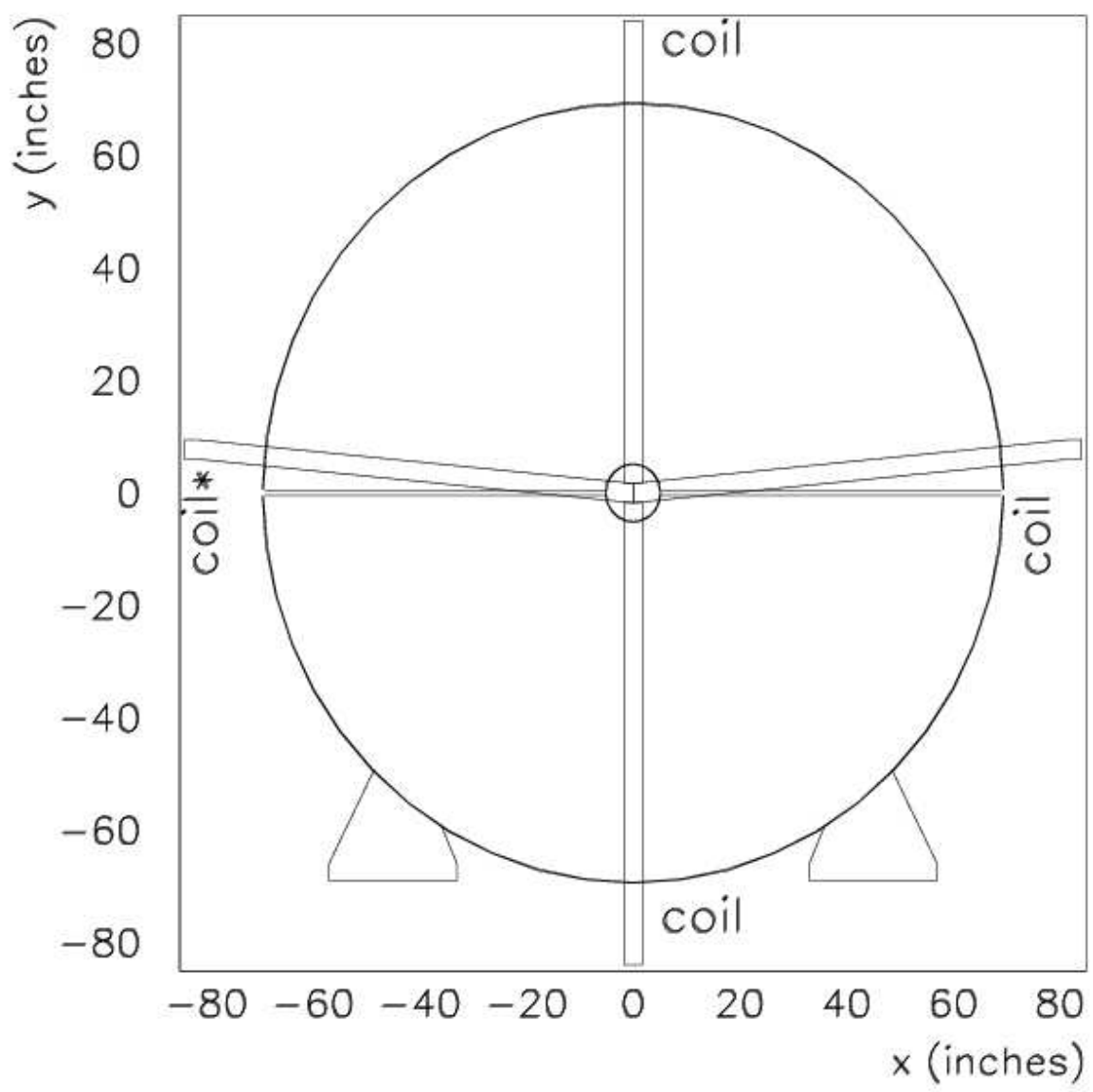

Figure 2.5: Cross section view of a NuTeV toroid magnet with four coils.

shorted to ground, and had to be disconnected. The effect of the burnt-out coil on the magnetic field of the toroid spectrometer is shown in Figure 2.7. This asymmetry in the magnetic field was included in $\mathrm{NuTeV}$ analysis software [30]. 


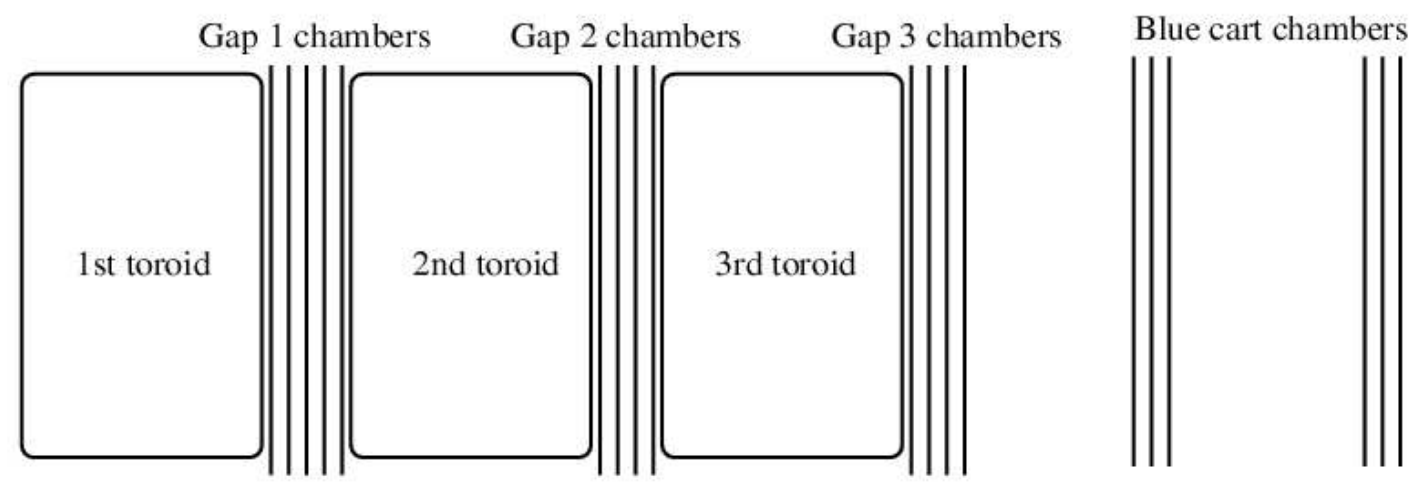

Figure 2.6: Side view of the $\mathrm{NuTeV}$ spectrometer with three toroid magnets and five sets of drift chambers.

\subsection{NUTEV BEAMLINE}

Neutrinos emerge from decay of the secondary pions and kaons produced in the interaction of $800 \mathrm{GeV}$ protons with a low- $Z \mathrm{BeO}$ target. The layout of the neutrino beamline is sketched in Figure 2.8. High energy protons from the Tevatron were obtained through the following steps:

- A dense plasma of hydrogen interacts with a cesium cathode producing $H^{-}$ions.

- A Cockcroft-Walton electro-statically accelerates the ions to $750 \mathrm{KeV}$.

- Ions are injected into a $79 \mathrm{~m}$ long linear accelerator (Linac) reaching energies of $400 \mathrm{MeV}$.

- $H^{-}$are passed through a carbon foil which strips the electrons resulting in a proton beam which is injected at a rate of $15 \mathrm{~Hz}$ to the Booster.

- The Booster, a $70 \mathrm{~m}$ radius synchrotron, accelerates protons to $8 \mathrm{GeV}$.

- The proton beam is sent to the Main Ring, an $1 \mathrm{~km}$ radius synchrotron which accelerates protons to $150 \mathrm{GeV}$ before being injected into the Tevatron. 


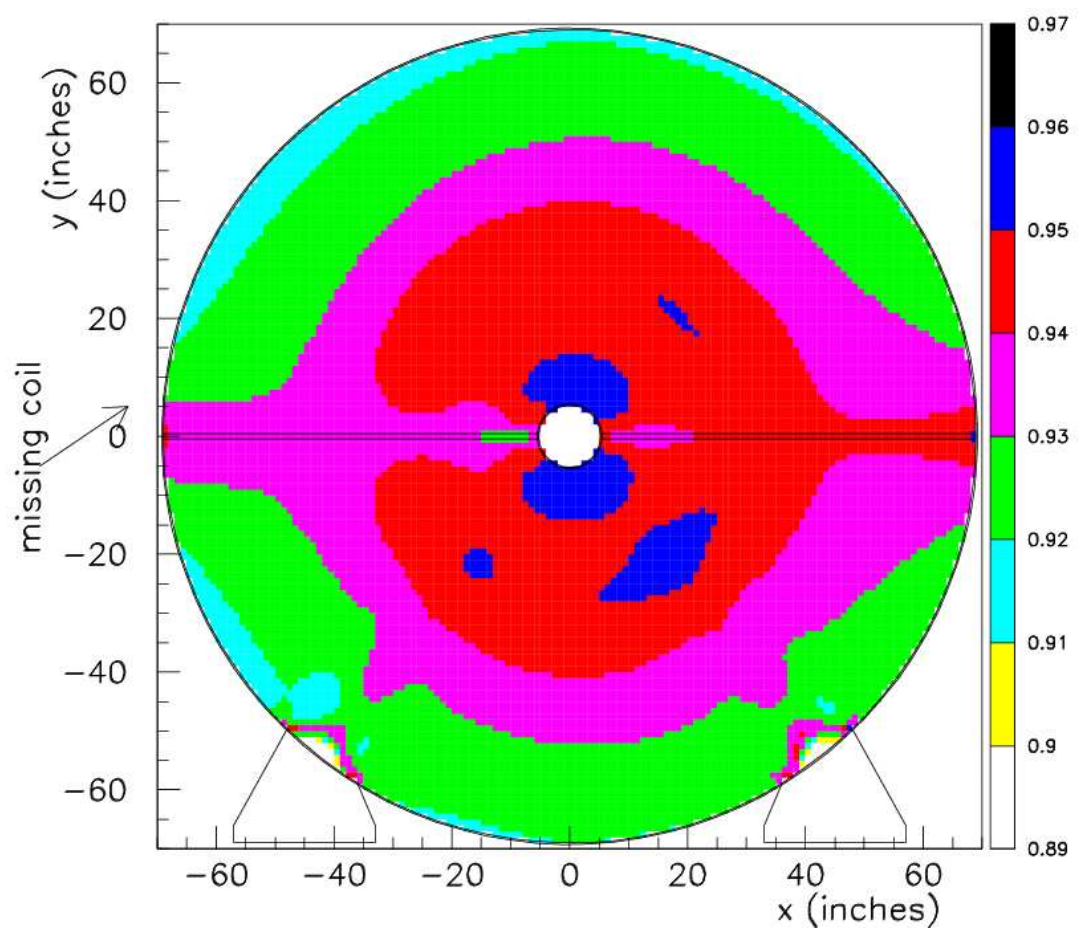

Figure 2.7: The effect of the burnt-out coil on the magnetic field of the toroid spectrometer from an ANSYS simulation. The ratio of 3 coil field to 4 coil field is shown. The burnt coil is on the left.

- Tevatron accelerates protons to $800 \mathrm{GeV}$. The beam is then directed to various experiments through a switch-yard.

The protons are extracted in one minute cycle of fast and slow spills. Figure 2.9 shows the structure of the beam timing. The fast spills consist of five $0.5 \mathrm{~ms}$ pings separated by $5 \mathrm{~ms}$. The fast spills allow $\mathrm{NuTeV}$ to minimize the contamination from cosmic rays.

The fast pings are directed into a $33 \mathrm{~cm}$ long BeO target producing a secondary beam of mesons. This beam enters into NuTeV's Sign Selected Quadrupole Train (SSQT) [31]. The SSQT is one of two key features of the $\mathrm{NuTeV}$ experiment. It provides separate high purity $\nu$ and $\bar{\nu}$ beams through a series of magnets which directs the desired charge of secondaries 


\section{Main Ring $150 \mathrm{GeV}$}

\section{Tevatron}

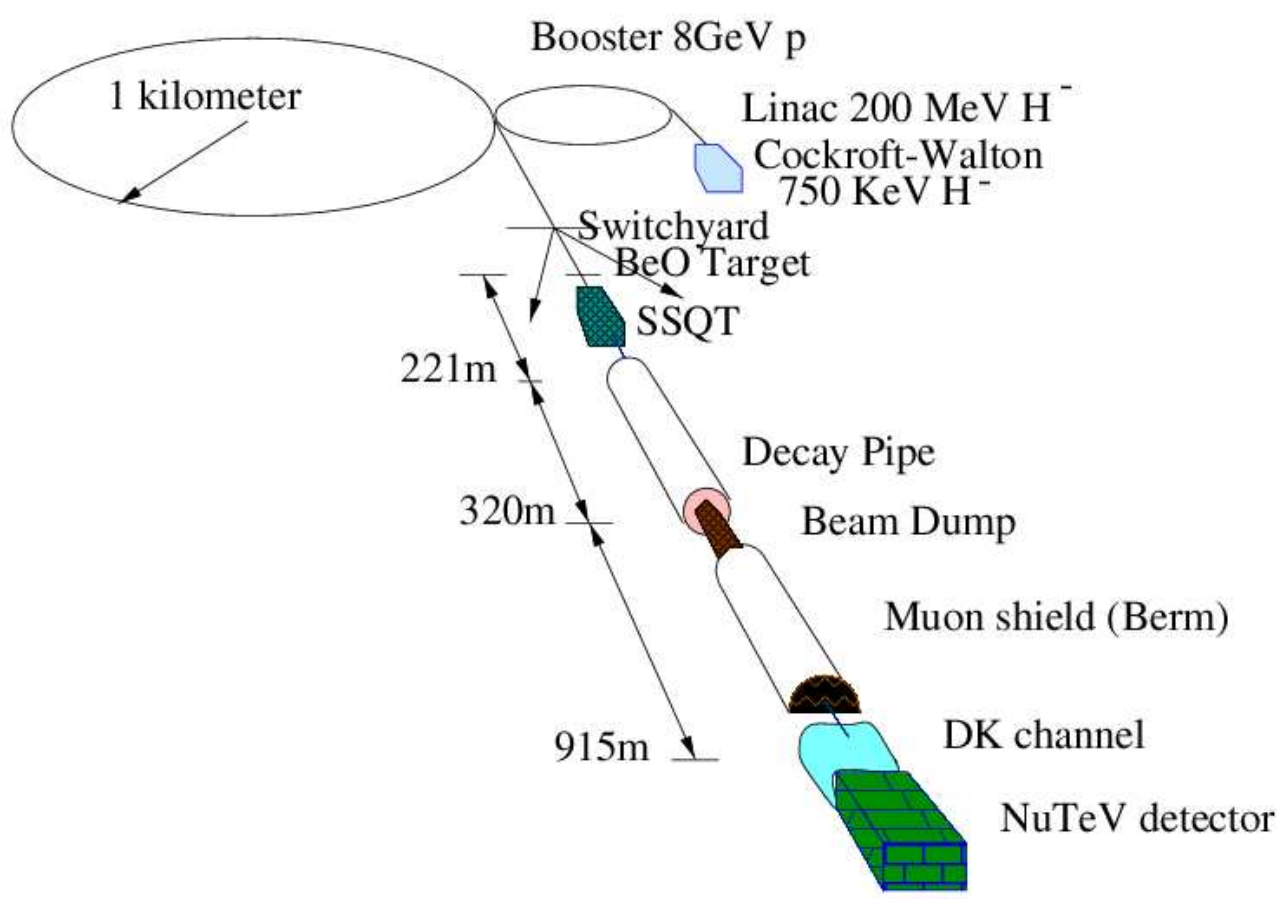

Figure 2.8: Schematic view of the Fermilab Neutrino beamline

("right-sign" mesons) into a decay space. The schematic view of the SSQT is shown in Figure 2.10. Undesired charged ("wrong-sign") particles are bent away to hit the beam dumps using the first dipole. Neutral particles go forward and are aimed away from NuTeV's downstream detector. Desired charged ("right-sign") mesons are directed at a 6 mrad angle towards the $\mathrm{NuTeV}$ detector. On their way, a few percent decay in-flight in a $300 \mathrm{~m}$ decay region as

$$
\begin{aligned}
\pi^{+(-)} & \rightarrow \mu^{+(-)}+\nu_{\mu}\left(\bar{\nu}_{\mu}\right) \\
K^{+(-)} & \rightarrow \mu^{+(-)}+\nu_{\mu}\left(\bar{\nu}_{\mu}\right)
\end{aligned}
$$

producing muons and neutrinos (dominant processes which produce $\left.\nu_{\mu}\left(\bar{\nu}_{\mu}\right) \mathrm{s}\right)$. Following the decay region is $900 \mathrm{~m}$ of earth and steel in which the un-wanted products of the decay (i.e. 


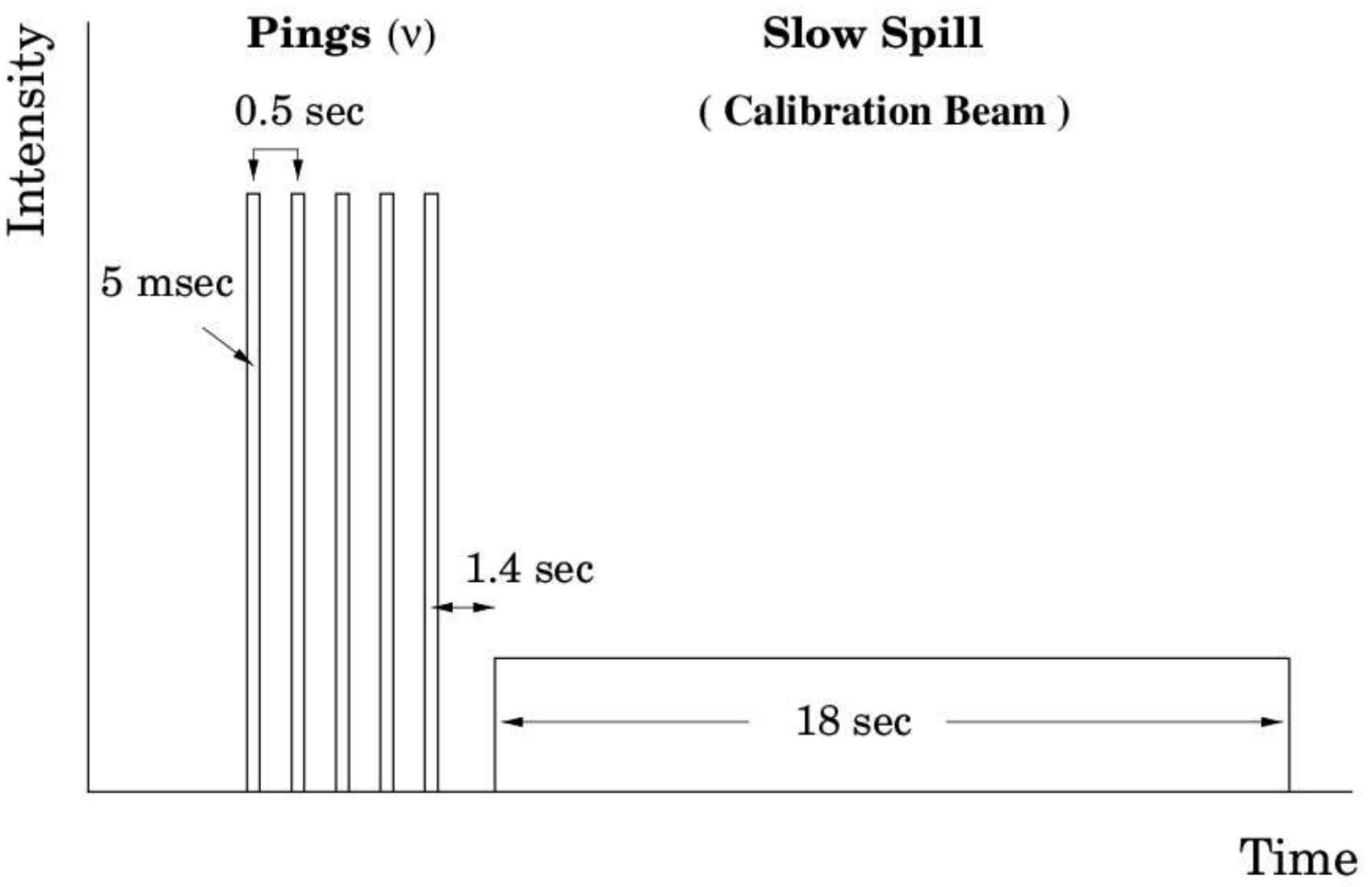

Figure 2.9: Structure of the beam timing. One cycle of $60.1 \mathrm{~s}$ is divided into fast and slow spills. Neutrinos are generated in five fast spills, the slow spill is dedicated to the calibration beam.

muons) are stopped. Therefore, NuTeV produces high purity neutrino or antineutrino beams. The contamination of antineutrinos in the neutrino beam (neutrino mode) is $0.03 \%$, and neutrinos in the antineutrino beam is $0.4 \%$. To reduce the electron-neutrino contamination of the final beam, the primary proton beam is not aimed in the direction of the NuTeV detector. Therefore, the dominant source of $\nu_{e}\left(\bar{\nu}_{e}\right) \mathrm{s}$ in the beam are from decay processes such as

$$
K^{+(-)} \rightarrow \pi^{0}+e^{+(-)}+\nu_{e}\left(\bar{\nu}_{e}\right)
$$






Figure 2.10: A sketch of NuTeV'Sign Selected Beam. A series of magnets select the desired charge of the secondaries.

The electron-neutrino contribution to the beam is $1.7 \%$ in neutrino mode and $1.6 \%$ in antineutrino mode. To predict various sources of neutrinos, a detailed beam Monte Carlo was used $[23,25]$.

Figure 2.11 shows flux spectra for neutrinos and antineutrinos in the NuTeV detector as function of neutrino energy. The first peak corresponds to neutrinos produced from pion decays, the second peak to neutrinos from kaon decays. The contaminations from wrong-sign and electron neutrinos also are shown.

\subsection{DATA ACQUISITION AND EVENT TRIGGERING}

A Data Acquisition System (DAQ) selects neutrino events, converts raw electronic signals into recordable data, and writes neutrino events to storage. These events are analyzed and 

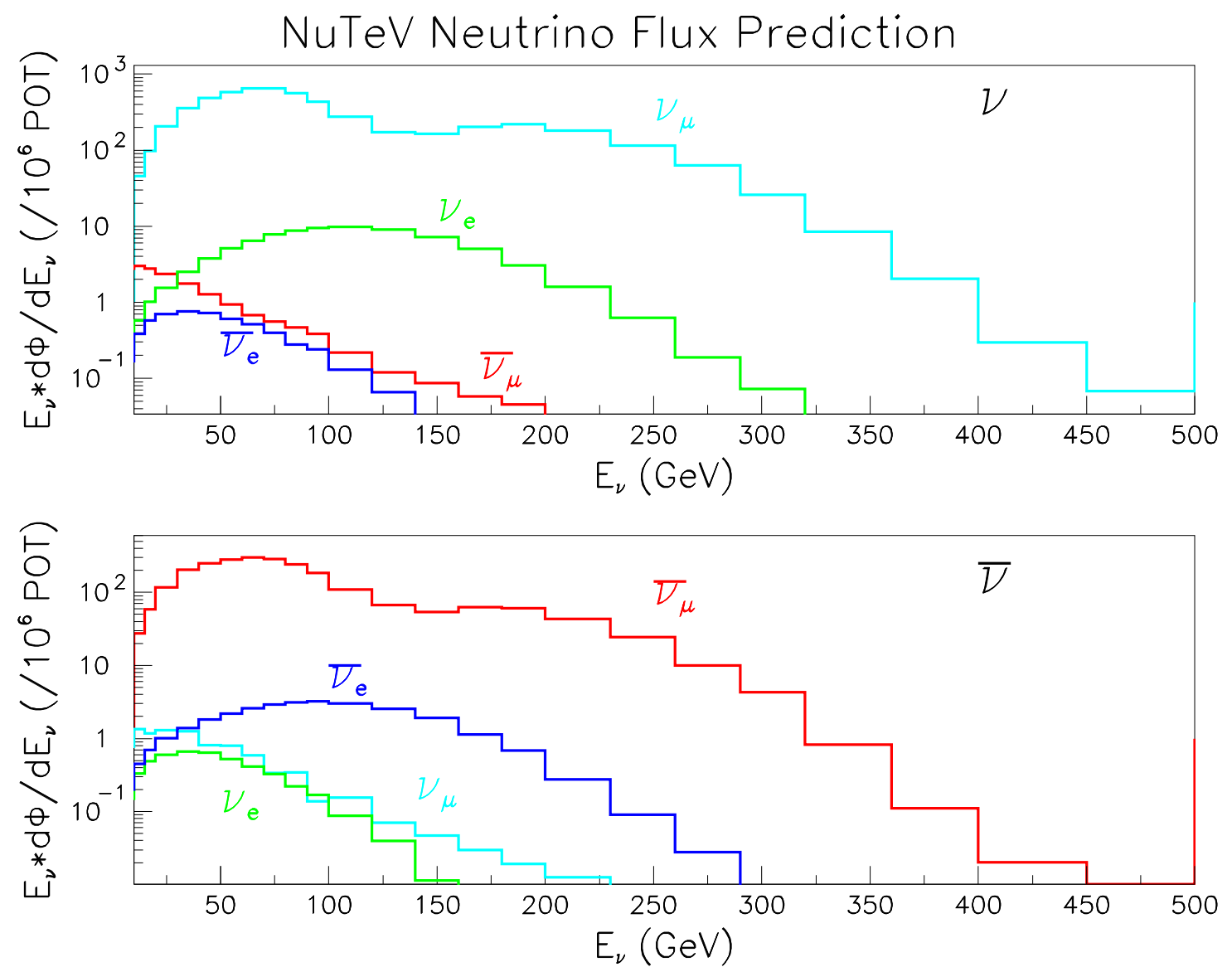

Figure 2.11: Flux prediction of neutrino beam for neutrino (top) and antineutrino (bottom) modes.

converted to physical quantities such as muon energy, muon angle, and hadronic shower energy.

\subsubsection{Readout Electronics}

Raw electronic signals from the phototubes of scintillation counters are in form of pulse heights $(\mathrm{PH})$. This information is used in the logic of the trigger system. The phototube signal is digitized by a readout system of Analog-to-Digital Converters (ADC) into ADC 
counts. Scintillation counters are registering pulses from wide energy ranges of muons and hadron showers, i.e. a typical muon deposits about $0.2 \mathrm{GeV}$ per counter. The readout system is divided into ADC channels of different sensitivities as:

- LOW: corresponds to un-amplified signal from a single phototube $(84$ counters $\times 4$ PMTs LOW ADC counts);

- COMBINATION LOW (COMBLO): is the sum of un-amplified signals from all 4 PMTs in a counter ( 84 COMBLO ADC counts);

- HIGH: is the $10 \times$ amplified COMBLO signal $(10 \times$ COMBLO ADC counts);

- SUPERLOW: is the sum of attenuated signals from 8 PMTs from different counters each separated by 10 counters.

A typical muon passing through the center of the counter will produce about 2 LOW, or 8 COMBLO, or $80 \mathrm{HIGH}$, or 0.2 SUPERLOW ADC counts. Figure 2.12 shows the readout for a single counter. The signal from an energy deposition which saturates the HIGHs can be measured by LOWs. If a strong shower saturates the LOWs, then the SUPERLOWs are used. The signal from a muon is too small to be measured by LOWs, hence HIGHs are used. COMBLOs are used to form triggers.

The raw signals from phototubes are duplicated through a fan-out device. The signal is discriminated and its timing is recorded in a time-to-digital converter (TDC). The discriminated signal ("bits") are constructed as follows:

- SBIT: Amplifies the signal from COMBLOs by 100 times, then discriminates it with a $150 \mathrm{mV}$ threshold. The SBITs are a single charged particle indicator from a single counter.

- TBIT: Amplifies the signal from COMBLOs by 100 times, then discriminates it with a $450 \mathrm{mV}$ threshold. The TBITs is a multiple charged particle indicator from a counter (should not fire for a single muon). 


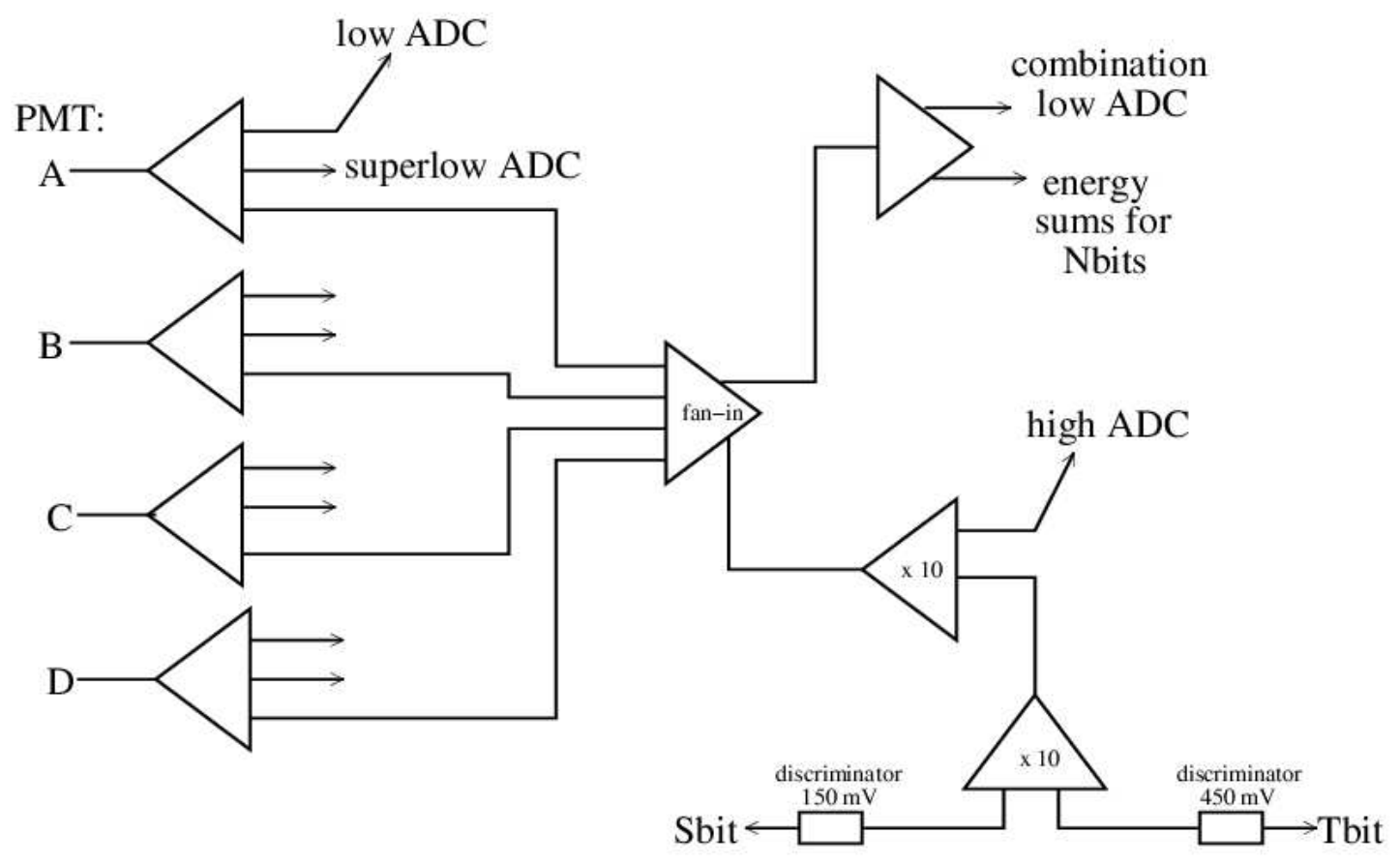

Figure 2.12: Configuration for the readout electronics of a single counter scintillation counter.

- NBIT: Sums un-amplified signals counters from COMBLOs of 8 consecutive counters, then discriminates it with a $55 \mathrm{mV}$ threshold.

\subsubsection{Event Triggers}

Twelve neutrino event triggers are formed from different combination of the digitized signals. The triggers are designed to select a particular event signature in the detector and to distinguish neutrino events from background and noise.

- TRIGGER 1 is designed to identify charged-current events which penetrate into toroid.

The trigger is fired for one of these two scenarios:

(A) There are hits at least in two of the last four counters and in two toroid gaps.

(B) There are hits at least in two of the last four counters, in two upstream counters 
(9-12), and in the first toroid gap only.

Trigger 1 was used in the selection criteria for the charged-current cross section analysis.

- TRIGGER 2 is designed to identify neutral-current events which deposit only hadronic showers. The trigger is fired if 8 consecutive counters have at least $5 \mathrm{GeV}$.

- TRIGGER 3 is used to select charge-current events in which a muon either stops in the target, or exits out the side. This trigger requires 16 counters to pass the SBIT threshold and at least $4 \mathrm{GeV}$ total energy deposited in any 8 consecutive counters.

- TRIGGER 4 is used to measure the TRIGGER 1 inefficiency.

- TRIGGER 5 is used for calibration beam running. No detection requirements.

- TRIGGER 6 is used to select the muons produced upstream of the detector. A hit in each target carts and hits in the same quadrant of each toroid gap are required. It is used for drift chambers alignment and counter calibration.

- TRIGGER 8 is designed to select cosmic ray muons. It requires hits in at last 40 semi-consecutive counters and in first toroid gap.

- TRIGGER 9 is designed to select possible neutral heavy lepton candidates which deposit energy only in the most upstream section of the detector..

- TRIGGER 10 is used to measure the in-spill pedestal.

- TRIGGER 11 defines events which shower in the toroid.

- TRIGGER 12 is designed to monitor the detector with no activity.

A veto system, composed of a wall of scintillation counters, is situated upstream of the detector to remove charged particles produced upstream of the detector. The first four triggers require not to have a veto signal. Table 2.2 shows number of neutrino events which fulfilled each trigger requirement (an event can satisfy more than one trigger). 
Table 2.2: Number of neutrino and antineutrino events for each trigger

\begin{tabular}{|l|c||c|c|}
\hline \hline Trigger & Name & $\nu$ events & $\bar{\nu}$ events \\
\hline \hline $\mathbf{1}$ & Charged-Current & 2574783 & 914886 \\
$\mathbf{2}$ & Neutral-Current & 5445024 & 2804981 \\
$\mathbf{3}$ & Penetrating Muon & 4306217 & 3065412 \\
$\mathbf{5}$ & Test Beam & 17000000 \\
$\mathbf{6}$ & Straight Through Muon & 1712591 & 735222 \\
$\mathbf{9}$ & Neutral Heavy Lepton & 1334675 & 1616637 \\
$\mathbf{1 0}$ & Straight Through Muon & 189885 & 183794 \\
$\mathbf{1 1}$ & Toroid Pedestal & 785040 & 153461 \\
\hline \hline
\end{tabular}

\subsection{EVENT RECONSTRUCTION}

The goal of the event reconstruction is to convert raw signal data into relevant physical quantities such as hadronic energy $\left(E_{\text {had }}\right)$, muon energy $\left(E_{\mu}\right)$, and muon angle $\left(\theta_{\mu}\right)$ at the vertex. ADC information is converted into pulse heights for each counter to determine the $z$-position of the interaction from the location of the first hit counter. The profile of energy deposition determines the length of the shower region which is used to find $E_{\text {had }}$. The track information is used to determine $\theta_{\mu}$. The muon's path in the toroid can be fitted to find the muon momentum. This is described in detail bellow.

\subsubsection{Pulse Height}

In a charged current neutrino interaction, a neutrino strikes a constituent quark inside a nucleon via a $W$ boson. The struck quark undergoes strong interactions, and the nucleon is 
fragmented into a number of hadrons, mostly pions, but also kaons, protons, neutrons and other baryons. The energy of the hadronic shower is totally absorbed by the calorimeter, but only about $3 \%$ of it is detected by scintillation counters (the rest is deposited in the steel). High energy particles deposit the same amount of energy in the calorimeter counters through ionization, referred to as minimum-ionizing-energy (MIE). The energy deposition is measured in units of minimum-ionizing-particles (MIPs), i.e. the amount of energy measured in a single scintillator divided by the amount of MIE. The definition of one MIP used in $\mathrm{NuTeV}$ is the truncated mean energy deposited by a $77 \mathrm{GeV}$ muon in one counter. The response of each counter is subject to variation in gain over time due to scintillation oil aging, PMT gain change and electronics response. The muon energy loss is well understood, therefore it is used as a calibration tool. It is described by the Landau distribution [32] characterized by a sharp peak due to the ionization losses of the muon, followed by a long tail due to the catastrophic energy losses such as $e^{+} e^{-}$pair production and bremsstrahlung (see Figure 2.13). NuTeV has an excellent calibration source to track counter gains: straightthrough muons produced upstream of the $\mathrm{NuTeV}$ detector which share the same time gate with actual neutrino beam and produce a similar illumination of the detector (TRIGGER 6 muons). This sample is used to monitor the position and time dependencies of each counter gain.

The energy deposited in a single counter $i, E_{c n t r}(i)$, registers electronic signals as the ADC pulse heights, $P H(i)$, which is modified by a number of factors as

$$
E_{c n t r}(\mathrm{MIPs})=\frac{h(i) G(i, t) P H(i)}{\operatorname{Map}\left(i, V_{x}, V_{y}, t\right)} .
$$

$\operatorname{Map}\left(i, V_{x}, V_{y}, t\right), G(i, t)$ and $h(i)$ are the counter map correction $\left(V_{x}, V_{y}\right.$ are the transverse vertex of the event), the counter gain and the relative hadron gain function, respectively, which are discussed below. The energy deposited by a charge particle in a single counter $i$ is 




Figure 2.13: A typical energy deposition of muons traversing one scintillation counter in units of ADC counts. Plot taken from Reference [28].

measured from the pulse height, $P H(i)$, which is registered in either LOW or HIGH channels: if the number of ADC counts in the LOW channel is less than 35 the HIGH channel is used (muons); if one of the LOW channels is saturated (more than 1900 ADC counts), the SUPERLOW channel is used. For example, a muon passing through the center of the counter deposits about 30 photoelectrons, which corresponds to 80 ADC counts in the HIGH channel. The pedestals (detector response when no trigger was fired) are removed from the pulse heights.

The counter gain factor, $G(i, t)$, converts the pulse height response in ADC counts into MIPs. The counter response to muons is calculated from the truncated mean of the muon 
distributions [28]. This mean energy loss defines the conversion from pulse height (registered in high ADC counts) to MIPs for each counter.

The relative response of each counter varies in the geometry and over time. The relative hadron gain function, $h(i)$, takes these variations and non-uniformities into account (i.e. variations in steel thickness or water bags).

The response of each counter depends on the location of the traversing muon. The position dependence of the muon response for each counter is determined by dividing the counter into a grid and measure the energy response in each section of the grid, as shown in Figure 2.14. This Figure shows that a muon passing near the center of the counter produces fewer ADC counts than a muon passing near the corners where the PMTs are located. The pulse heights are corrected for these variations. The position-dependent correction $\operatorname{Map}\left(i, V_{x}, V_{y}, t\right)$ is determined by the ratio of the counter response at the $(x, y)$ position to the counter response at the center $(0,0)$. The time variations in the counter are measured at the center of each counter where the event population is the highest.

\subsubsection{Raw Event Variables}

Figure 2.15 presents a typical event display of a charged current neutrino interaction in both views $(X$ and $Y)$. In the figure, a neutrino entering from the left interacts inside the target producing a hadronic shower and a muon detected by the target scintillators and drift chambers. The RUN and EVENT information are recorded in the header of each event, as well as which TRIGGER fired for that event. The red lines correspond to the pulse heights of the scintillation counters: the hadron shower is characterized by the spikes, and the muon by a nearly constant signal as it traverses the detector. The corrected pulseheight can be used to identify the interaction region along the $z$ axis of the detector. The beginning of the interaction is marked by several large pulse heights that define the position of the interactions. The variable PLACE is defined as the most upstream counter of two 




Figure 2.14: Average counter response to muons as a function of the position. The coordinates are normalized to the half width of the counter $(1.5 \mathrm{~m})$ on both axes.

consecutive counters with at least 4 MIPs. The variable that defines the end of the shower is SHEND. It corresponds to the first of three consecutive counters with less than 4 MIPs. An illustration of the event length determination for charged current neutrino is shown in Figure 2.16. Using these definitions, the reconstruction of data can be separated into three regions which helps determine the physical quantities such as $E_{h a d}, E_{\mu}$, and $\theta_{\mu}$ :

1. Shower Region: extends between PLACE and SHEND-5. This is where hadronic energy is determined.

2. Target Muon Track: follows the shower region from SHEND-6 to counter 1. It is used to determine muon energy loss in the target. 




Figure 2.15: A $\nu_{\mu}$ event display for a typical charged current neutrino interaction.

3. Toroid Spectrometer: muon energy is determined by fitting the muon track

Next, we present the reconstruction of the hadronic energy, muon energy, and muon angle.

- Reconstruction of the hadronic energy:

Hadronic energy is determined by a simple sum of the counter pulse heights within the shower region with energy deposited by the primary muon subtracted,

$$
E_{\text {had }}(\mathrm{GeV})=C_{\pi} \sum_{i=S H E N D-5}^{P L A C E}\left(E_{\text {cntr }}(i)-E_{\mu}^{\text {loss }}(i)\right)
$$

where $C_{\pi}$ is the hadron calibration constant that converts MIPs to GeV and it is discussed in Sect. 2.5. Muon energy in the shower region is not directly measured. Instead, a 




Figure 2.16: Pulse height of an event (in MIPs) versus counter number. Muon track and shower regions are shown.

parametrization of the average energy loss for a minimum ionizing particle (in MIPs) is used:

$$
E_{\mu}^{\text {loss }}(i)=0.9315+0.02359 \log E_{\mu}^{e s t}
$$

where $E_{\mu}^{e s t}$ is the estimated energy loss of the muon (in $\mathrm{GeV}$ ).

\section{- Reconstruction of the muon angle:}

The muon angle at the vertex is determined by fitting the muon track (obtained from the recorded muon hits in the drift chambers) near the vertex. Because no magnetic field is 
present in the target, the muon track in the target is straight. In the track fitting procedure, a line segment is chosen in the calorimeter to fit the hits in the most downstream drift chambers. This straight-line segment is extrapolated upstream to PLACE, but due to the hadron shower the hits immediately after PLACE are not used for tracking. The number of drift chambers nearest the vertex position that are omitted from the tracking procedure depends on the hadron energy: higher energy hadron showers traverse more drift chambers. The multiple Coulomb scattering (MCS) effects complicate the muon track reconstruction. A particle with momentum $P_{\mu}$ traversing a distance $L$ in a material with radiation length $\lambda_{\text {rad }}$ will suffer a mean Coulomb scattering deflection

$$
\sigma_{\theta}^{M C S}=\frac{0.015}{P_{\mu}} \sqrt{\frac{L}{\lambda_{\text {rad }}}} .
$$

Therefore the muon angle measurement is correlated with muon momentum, and the fitting procedure is iterated.

\section{- Reconstruction of the muon energy:}

The energy of the muon is reconstructed in three parts: muon deposited within the

hadron shower, $E_{\mu}^{\text {shower }}$, muon deposited in the calorimeter beyond hadron shower, $E_{\mu}^{\text {target }}$ (i.e. $d E / d x$ ), and muon energy measured at the front face of the steel in the toroid spectrometer, $E_{\mu}^{F F}$ :

$$
E_{\mu}(\mathrm{GeV})=E_{\mu}^{\text {shower }}+E_{\mu}^{\text {target }}+E_{\mu}^{F F}
$$

The muon energy loss in the shower region cannot be determined due to absence of position information in the shower region. Therefore, a parametrization of the most probable $d E / d x$ loss per counter (in $\mathrm{GeV}$ ) is used,

$$
E_{\mu}^{\text {shower }}=\sum_{i=S H E N D-5}^{P L A C E}\left(0.1668+0.00037 E_{\mu}^{e s t}(i)\right) / \cos \theta_{\mu}
$$


where $E_{\mu}^{e s t}(i)$ is the estimated muon energy and $\theta_{\mu}$ is the muon angle. Muon energy loss beyond the shower region is determined from the sum of corrected PHs in each counter beyond the shower region as,

$$
E_{\mu}^{\text {target }}(\mathrm{GeV})=\sum_{i=1}^{S H E N D-6} E_{\mu}^{c n t r}(i)
$$

where

$$
E_{\mu}^{c n t r}(i)= \begin{cases}C_{\mu}\left(E_{\mu}\right) E_{c n t r}(i), & \text { for small PHs } \\ C_{\mu}\left(E_{\mu}\right)+C_{e}\left(E_{c n t r}(i)-1\right), & \text { for large PHs }\end{cases}
$$

For small pulse heights ( $<5$ MIPs), the muon energy loss is assumed to be from ionization processes with a muon energy dependent conversion factor, $C_{\mu}\left(E_{\mu}\right)$. For high pulse heights (> 5 MIPs), the muon energy loss is attributed to catastrophic processes (i.e. pair production, Bremsstrahlung) which produce an electromagnetic cascade, hence, requiring an electromagnetic calibration factor, $C_{e}$. The calibration factors, $C_{\mu}\left(E_{\mu}\right)$ and $C_{e}$, which convert MIPs into GeVs are discussed in Sect. 2.5.

The muon momentum at the front face of the toroid spectrometer is obtained from the bend of the muon track in the magnetic field of the toroid. In a constant magnetic field in vacuum, the bend angle is given by relation

$$
\theta_{\text {bend }}=\frac{0.3 B L_{\mu}}{P_{\mu}}
$$

where $P_{\mu}$ is the muon momentum and $L_{\mu}$ is the length of the track in the magnetic field. The algorithm for muon momentum determination is as follows: a projected track is obtained from the measured muon angle in the target and by using a model of the magnetic field; the hits in the toroid chambers are fit to this projected track. The MCS effects and muon energy loss in the steel are included in the track fitting procedure. 


\subsection{CALIBRATION}

The measurements from previous neutrino experiments were dominated by the experimental uncertainties due to energy scales (muon and hadronic) [33], [34]. There are two key features that enable $\mathrm{NuTeV}$ to be the most precise neutrino experiment to date. One is the SSQT which was presented in Sect.2.2, and the other feature is NuTeV's calibration beam which considerably improves its knowledge of the energy scales, presented next. Here we outline some characteristics of the $\mathrm{NuTeV}$ calibration beam, the reader is referred to Ref. [28] for more details on the calibration and response measurements.

\subsubsection{In Situ Calibration Beam}

The NuTeV detector was continuously calibrated with a charged particle beam. Calibration beam running was concurrent with neutrino data taking. The calibration data stream was separated from the neutrino data by $1.4 \mathrm{~s}$ within the same accelerator cycle. The slow spill in Figure 2.9 corresponds to calibration beam running, while the fast spill to neutrino running. Protons during the slow spill were passed through an aluminum block producing secondaries which were focused and selected for momentum through a series of collimators and dipoles. The selected particles (i.e. electrons, muons, and hadrons over a wide range of energy from 4.8 to $190 \mathrm{GeV}$ ) were then passed through a low mass spectrometer with a long lever arm. The schematic view of this spectrometer and calibration beam is shown in Figure 2.17. The spectrometer was instrumented with drift chambers, dipole magnets, a Čerenkov detector and an array of transition radiation detectors (TRDs) used for particle identification. The drift chambers are used to track the charged particles, the dipole magnets are adjusted so that the particles of the correct momentum are directed to $\mathrm{NuTeV}$ detector. The Čerenkov detector and the TRD are used to discriminate among electrons, pions, kaons and anti-protons when needed, otherwise they are rolled out of the beamline and replaced by 




Figure 2.17: A schematic view of the NuTeV Calibration Beam directed at 43 mrad angle and the low mass spectrometer with a long lever arm.

a vacuum pipe to reduce the multiple scattering. Figure 2.18 shows a clean identification for pions, kaons and anti-protons within the $50 \mathrm{GeV}$ hadron beam by the Čerenkov counter using $N_{2}$ gas at 160 torr pressure. The last dipole magnet of the calibration beam spectrometer could be steered to map the response of the over the front face of the detector.

The spectrometer measured energy with a resolution of $0.3 \%$ in an event-by-event basis. This was achieved by using precisely calibrated dipole magnets with $\int B d l$ known to better than $0.1 \%$ in the region traversed by the beam (i.e. "good field" region). A calibration beam trigger, which consists of two scintillation paddles, was used to maintain the $0.1 \%$ tolerance of the $\int B d l$. Also, the long distances of $83.3 \mathrm{~m}$ between the most upstream chamber and momentum analyzing magnets, and of $69.2 \mathrm{~m}$ between the most downstream chamber and magnets allowed a relative chamber alignment uncertainty of $1 \mathrm{~mm}$. This can be translated into $0.1 \%$ uncertainty in the beam momentum.

The absolute hadron energy scale of the calorimeter is determined by measuring its 


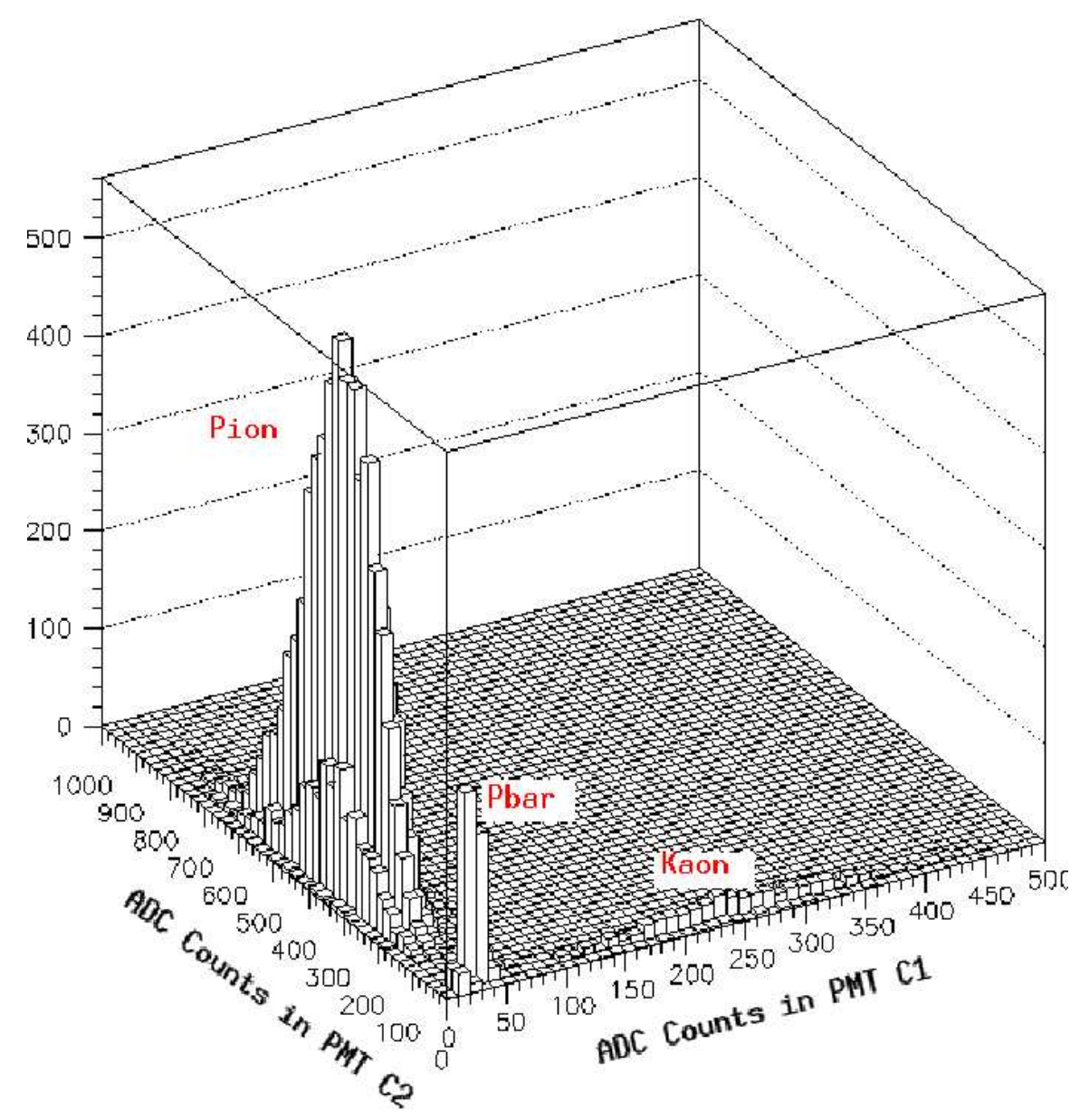

Figure 2.18: Particle identification among $\pi, K, \bar{p}$ at $50 \mathrm{GeV}$ hadron beam shown by PMT signals (C1 and C2, placed on opposite directions) of the Cerenkov counter with 160 Torr $N_{2}$ gas. Plot taken from Ref. [28].

response to single pion interactions. The calibration beam composition of hadrons has been studied in details. Table 2.3 summarizes particle composition in the hadron calibration beam for various energies. Electrons were removed from the low energy samples through cuts based on Čerenkov particle ID system. The response of the calibration beam was corrected for the contamination of pions from kaons and antiprotons using clean samples of each particle type 
Table 2.3: Particle composition in hadron calibration beam for various energies.

\begin{tabular}{|c|c|c|c|c|}
\hline $\mathrm{P}$ & Electron & \multicolumn{2}{|r|}{ Hadron $\left(\pi^{-}, K^{-}, \bar{p}\right)$ fraction $(\%)$} & correction \\
\hline 5 & 92 & 8 & & $-0.6 \pm 0.2$ \\
\hline 15 & 66 & 34 & $\left(\pi^{-}: 95.6 ; \quad \bar{p}+K^{-}: 4.1\right)$ & -0.22 \\
\hline 20 & $<1$ & $>99$ & $\left(\pi^{-}: 95.5 ; \quad \bar{p}+K^{-}: 4.5\right)$ & -0.15 \\
\hline 30 & $<0.25$ & $>99.75$ & $\left(\pi^{-}: 94.9 ; \quad \bar{p}+K^{-}: 5.1\right)$ & -0.14 \\
\hline 50 & 0 & $>100$ & $\left(\pi^{-}: 93.9 ; \quad \bar{p}: 3.0 ; \quad K^{-}: 3.1\right)$ & -0.017 \\
\hline 75 & 0 & $>100$ & $\left(\pi^{-}: 91.7 ; \quad \bar{p}: 3.2 ; \quad K^{-}: 5.1\right)$ & -0.010 \\
\hline 120 & 0 & $>100$ & $\left(\pi^{-}: 91 ; \quad \bar{p}: 2.8 ; \quad K^{-}: 6.2\right)$ & -0.002 \\
\hline
\end{tabular}

given by the Čerenkov counter information. It has been found that the showers from kaons agree with those from pions, while those from antiprotons give a higher response due to the $p \bar{p}$ annihilation processes. The correction factors for these effects on hadronic energy are estimated and are presented in Table 2.3 (see the last column). This effect is negligible for energies greater than $50 \mathrm{GeV}$ (i.e. has less than $0.03 \%$ effect).

\subsubsection{Calorimeter Calibration}

The reconstructed energy by calorimeter is proportional to the number of shower particles produced by the incident hadron. Therefore, the resolution function of the calorimeter for hadrons can be characterized by a Poisson distribution. The electromagnetic component of the shower causes the response to look Poisson-like at low energies and Gaussian at high energies as shown in Figure 2.19. 



Figure 2.19: Poisson fits to the ratio of measured hadron calorimeter energy to reconstructed calibration beam momentum distributions for $5 \mathrm{GeV}, 10 \mathrm{GeV}, 75 \mathrm{GeV}, 190 \mathrm{GeV}$ hadrons. 
The figure shows the calorimeter energy $E$ divided by the reconstructed calibration beam momentum $p$. The hadron conversion factor $C_{\pi}$ converts the pulse height MIP response of the detector to $\mathrm{GeV}$. It is determined by fitting the hadron energy dependencies of the average $E / p$ distributions for hadrons ranging from 10 to $190 \mathrm{GeV}$, as shown in Figure 2.20.

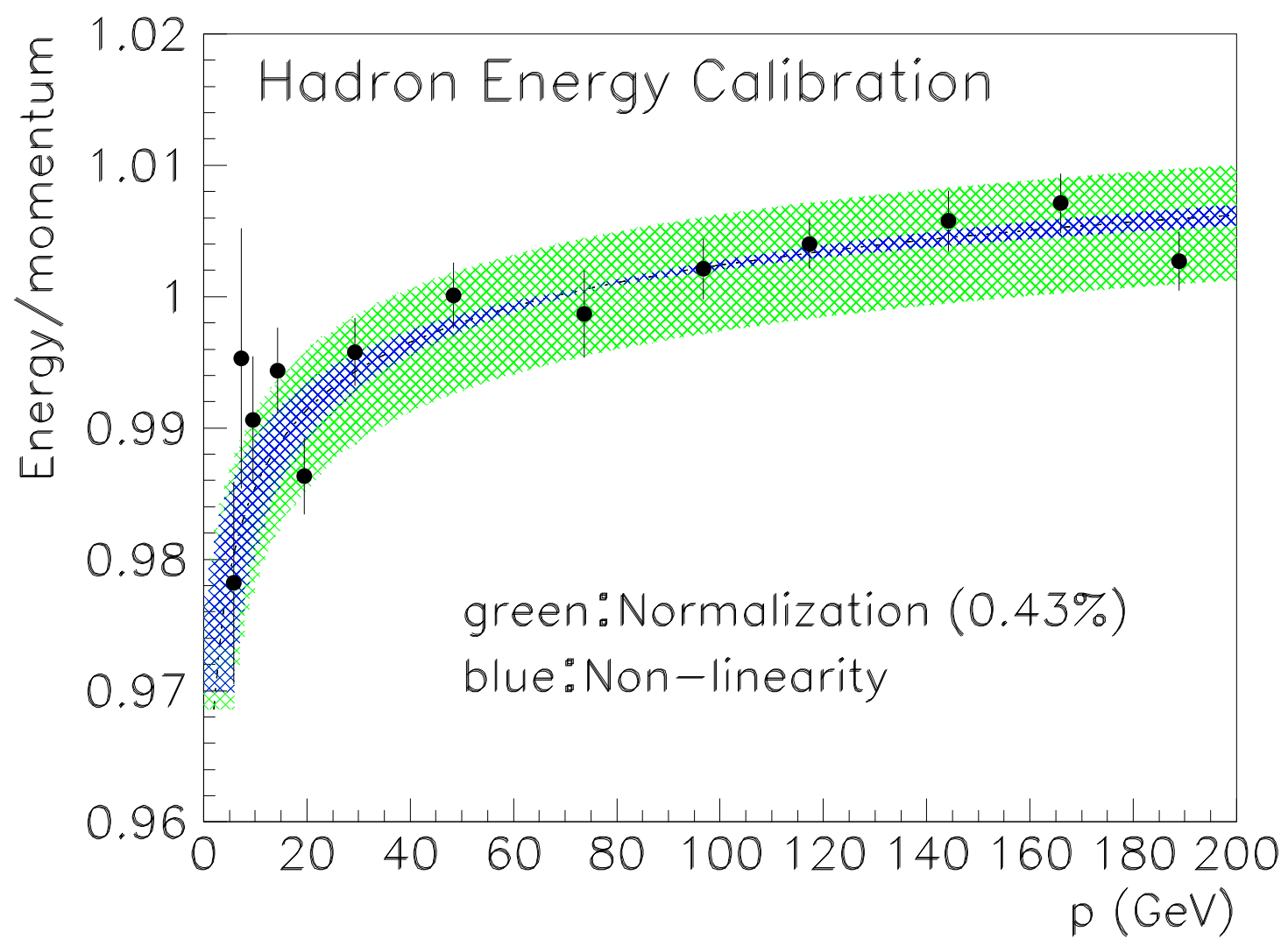

Figure 2.20: Ratio of hadron energy response over the calibration beam momentum and the comparison with the fit for the hadron non-linearity (the blue line - narrow band) of the calorimeter between 10-190 GeV. The green band (large band) reflects the overall uncertainty of $0.43 \%$ in the hadron energy scale.

The hadron showers have nearly the same probability of producing neutral pions $\pi^{0}$ as $\pi^{+}, \pi^{-}$. The $\pi^{0}$ particle decays into two photons that initiate electromagnetic cascade. This 
causes a non-linear response of the detector to hadron energy (i.e. there is an electromagnetic component in the shower). This non-linear response to hadrons in the calorimeter is taken into account. Thus, the calibration for a realistic hadronic shower that contains both the "pure" hadrons and electrons can be expressed as

$$
C_{\pi}=e f_{\pi^{0}}\left(E_{h a d}\right)+h\left[1-f_{\pi^{0}}\left(E_{h a d}\right)\right]
$$

where $h, e$ are the calibration constants for a "pure" hadronic shower, electromagnetic shower, respectively; $f_{\pi^{0}}$ is the fraction of $\pi^{0}$ produced in the shower (i.e., the fraction of electromagnetic energy in a hadronic shower). The hadron energy response is fitted assuming an energy dependent parametrization for $f_{\pi^{0}}$ given by Groom's parametrization (Ref. [35]),

$$
f_{\pi^{0}}\left(E_{h a d}\right)=1-\left(\frac{E_{h a d}}{A}\right)^{B}
$$

with $A=0.96 \mathrm{GeV}$ and $B=-.0184$. It yields a value of $e / h=1.079 \pm 0.011$ for the ratio of electron to hadron response. The non-linearity in the hadron energy response is found to be about $3 \%$. The green band (large band) in Figure 2.20 reflects the overall uncertainty of $0.43 \%$ in the hadron energy scale. For $C_{\pi}=0.212 \mathrm{GeV} / \mathrm{MIPs}$ the ratio of the mean energy response in the detector to the reconstructed hadron calibration beam momentum at $75 \mathrm{GeV}$ is $1.000 \pm 0.001$.

The energy resolution as function of energy is determined through a fit to Poisson-width using a standard form:

$$
\frac{\sigma(E)}{E}=A \oplus \frac{B}{\sqrt{E}} \oplus \frac{C}{E}
$$

where $A$ is a constant term associated with the calibration uncertainties, $B$ is the stochastic term associated with sampling fluctuations, and $C$ is the noise due to pedestal fluctuations (this term was removed later from the fit because data showed no evidence for noise). Figure 2.21 shows the fit to Poisson widths of $E / p$ distributions as function of energy. The energy 




Figure 2.21: Measured hadron resolution from fit to the width of Poisson-like distributions.

resolution of the calorimeter was found to be

$$
\frac{\sigma(E)}{E}=0.022 \pm 0.001 \oplus \frac{0.86 \pm 0.01}{\sqrt{E}}
$$

To study any possible difference between hadron showers produced by neutrino interactions and showers produced by single charged pions a GEANT-based Monte Carlo with LEPTO interaction generator was used Differences were found to be small. The overall energy scale is consistent with zero to better than $0.1 \%$ [28].

The electromagnetic conversion factor $C_{e}$ was determined from the calorimeter response to electrons using the electron calibration beam. Figure 2.22 shows the Poisson fits to the ratios of calorimeter electron energy to the calibration electron beam for 30 and $170 \mathrm{GeV}$. It was obtained a value of $C_{e}=0.195 \mathrm{GeV} / \mathrm{MIP}$ for electron calibration constant. The electron resolution is determined to be

$$
\frac{\sigma(E)}{E}=0.042 \pm 0.002 \oplus \frac{0.499 \pm 0.008}{\sqrt{E}} .
$$



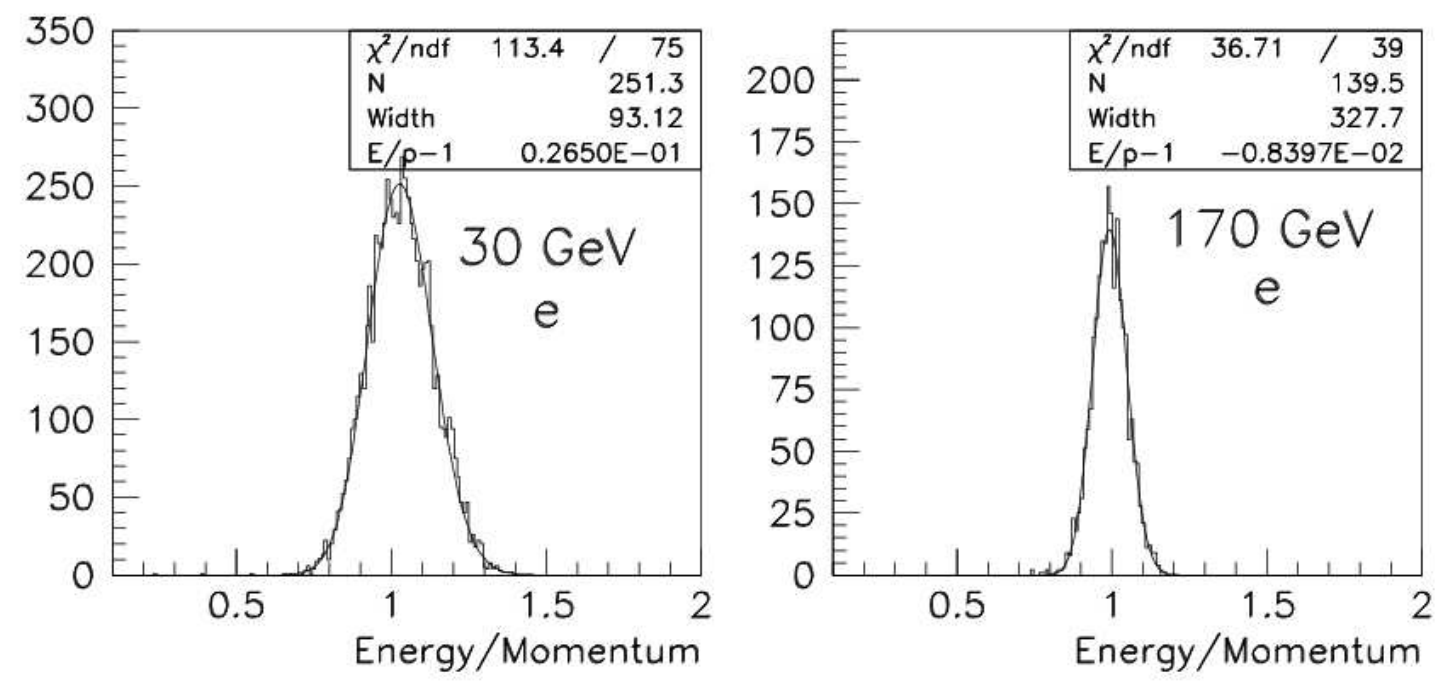

Figure 2.22: Poisson fits to the ratio of measured electron calorimeter energy to reconstructed calibration beam momentum distributions for 30 and $170 \mathrm{GeV}$ electrons.

The muon conversion factor $C_{\mu}$ from MIPs to $\mathrm{GeV}$ was determined from muon energy loss in the calorimeter which is attributed to ionization loss. The parametrization for $C_{\mu}$ is determined from fitting muon pulse height distribution as

$$
C_{\mu}\left(E_{\mu}\right)= \begin{cases}0.1419+0.004357 \log E_{\mu}, & E_{\mu}<60 \mathrm{GeV} \\ 0.1419+0.004357 \log (60)+0.00006822\left(E_{\mu}-60\right), & E_{\mu} \geq 60 \mathrm{GeV}\end{cases}
$$

\subsubsection{Toroid Response Calibration}

The muon energy calibration was performed using the calibration beam of muons at 50 and $100 \mathrm{GeV}$ scanning the entire face of the toroid. There were 45 calibration beam runs used in the muon energy calibration. For each test beam muon run a fit to the average ratio of the muon momentum measured by the calibration beam spectrometer to the muon 
momentum measured by $\mathrm{NuTeV}$ detector is determined (i.e. $P_{\mu}^{T B} / P_{\mu}^{D E T}$ ). Figure 2.23 shows the fitted mean location at the front face of the toroid for muon calibration beam runs. The

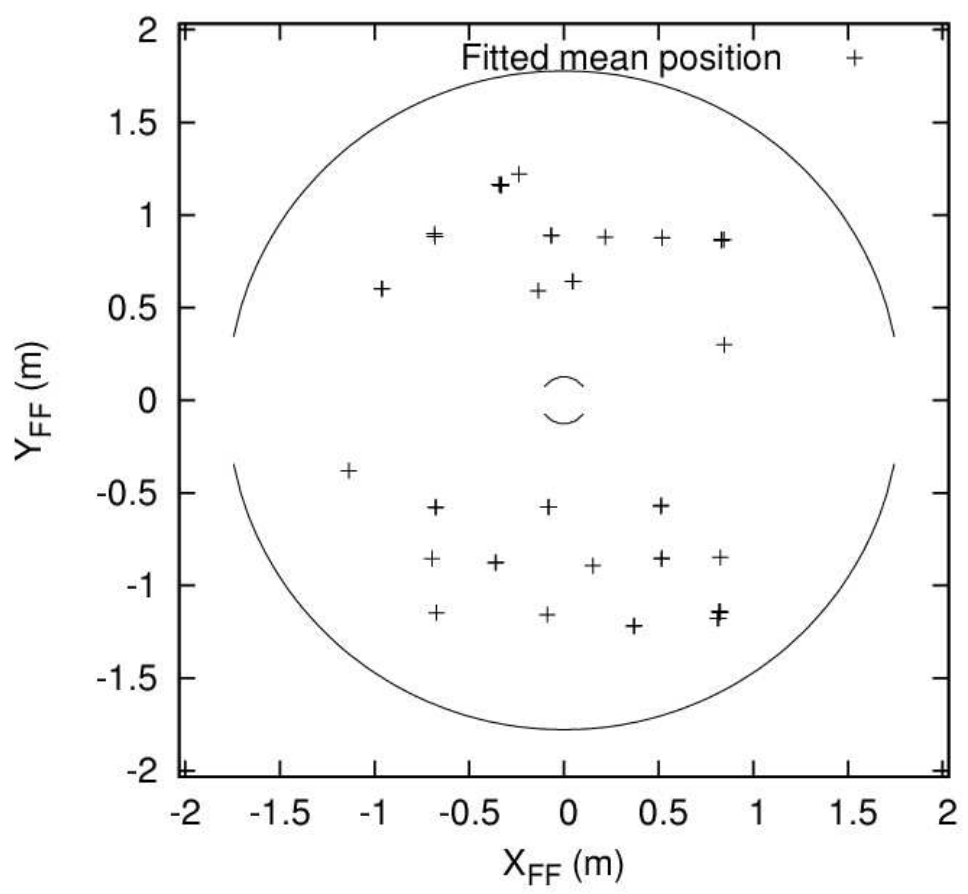

Figure 2.23: Location at the front face of the toroid of the fitted mean position of the ratio of the calibration beam muon momentum to the measured momentum by $\mathrm{NuTeV}$ detector for all 45 muon runs.

absolute muon energy scale uncertainty is given by the variance of these fitted means and is determined to be $0.7 \%[22]$.

The resolution for the toroid spectrometer is limited by the MCS effects in the steel toroids. A typical sigma for an angular deflection due to MCS is proportional to inverse muon momentum (see Eq. (2.8)). The uncertainty due to chamber resolution is constant. Therefore, the momentum resolution function is given by

$$
\frac{\Delta\left(1 / P_{\mu}\right)}{1 / P_{\mu}}=\frac{P_{\mu}^{T B}-P_{\mu}^{D E T}}{P_{\mu}^{D E T}},
$$


where $P_{\mu}^{T B}$ is the muon momentum measured by the test beam spectrometer, and $P_{\mu}^{D E T}$ is muon momentum distribution measured by the $\mathrm{NuTeV}$ detector. The resolution function of the muon spectrometer is fitted to the following parametrization:

$$
\frac{\Delta\left(1 / P_{\mu}\right)}{1 / P_{\mu}}=\exp \left[-\frac{1}{2} \frac{x^{2}}{\sigma_{\text {lead }}^{2}\left(P_{\mu}\right)}\right]+R_{\text {tail }}\left(P_{\mu}\right) \exp \left[-\frac{1}{2} \frac{\left(x-x_{\text {tail }}\left(P_{\mu}\right)\right)^{2}}{\sigma_{\text {tail }}^{2}\left(P_{\mu}\right)}\right]
$$

where $\sigma_{\text {lead }}\left(P_{\mu}\right)$ is the width of the leading Gaussian contribution due to MCS, and $R_{\text {tail }}\left(P_{\mu}\right)$, $\sigma_{\text {tail }}\left(P_{\mu}\right)$ and $x_{\text {tail }}\left(P_{\mu}\right)$ are the height, the width and the offset of the asymmetric tail of the resolution function. The width of the leading Gaussian has a small energy dependence and is parametrized as

$$
\sigma_{\text {lead }}\left(P_{\mu}\right)=\sqrt{A^{2}+\left(B P_{\mu}\right)^{2}}
$$

The tail parameters instead are parametrized with linear functions. The response of the detector to muon calibration beam at $100 \mathrm{GeV}$ is fitted using the above parametrization of the resolution function and is shown in Figure 2.24. The fit parameters for a muon momentum at $100 \mathrm{GeV}$ are given in Table 2.4 (see Ref. [22]).

\subsubsection{Muon Angle Calibration}

Muon angle at the vertex is determined by fitting the muon track near the vertex. The number of hits near the vertex is large due to the hadron shower which limits the tracking ability. Therefore, the resolution of the muon angle is limited by hadron energy and by uncertainty due to MCS. The muon angle measurement is correlated with muon momentum, therefore the procedure is iterated using GEANT hit level simulation [36] of the detector to parametrize the angular resolution function (see Sect. 3.2.3). 


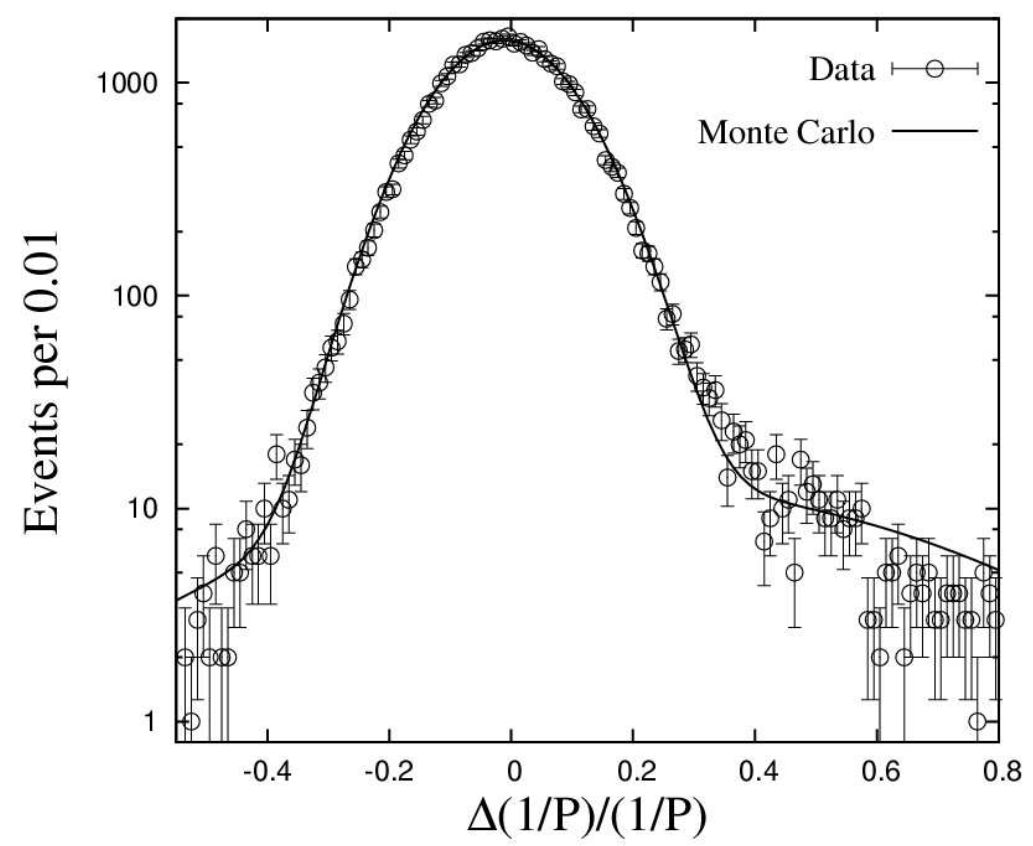

Figure 2.24: Fit to the detector response to muon calibration beam of $100 \mathrm{GeV}$. Plot taken from Ref. [22]

Table 2.4: Parameters of the muon energy resolution function for a muon momentum entering the toroid at $100 \mathrm{GeV}$.

\begin{tabular}{|l|l|l|}
\hline \hline$\sigma_{\text {lead }}(100)$ & width of leading Gaussian & 0.11 \\
$\sigma_{\text {tail }}(100)$ & width of the asymmetric tail function & 0.47 \\
$x_{\text {tail }}(100)$ & offset of the asymmetric tail & 0.18 \\
$R_{\text {tail }}(100)$ & normalization coefficient & 0.079 \\
\hline \hline
\end{tabular}




\subsection{EXTRACTION OF THE DIFFERENTIAL CROSS SECTION}

The strong coupling constant is determined from fits to the extracted neutrino-nucleon struc-

ture functions. However, the quantity which is directly measured by the experiment is the neutrino-nucleon differential cross section on iron.

Measured physical quantities are smeared by detector resolution effects and are also limited by detector acceptance. In order to account for these effects a Monte Carlo simulation is used which is meant to convert smeared measured distributions into true physical distributions. The simulation employs a complete description of the detector and a cross section model which is iteratively fitted to differential cross-section data. In this Chapter we summarize the mechanism of extraction of the differential cross section as it is schematically shown in Figure 3.1 (detailed description can be found in Ref. [22]). The reminder of this chapter presents the extraction of the structure functions which are used in determination of the strong coupling constant.

\subsection{DATA SELECTION}

Once data is reconstructed as described in the previous Chapter, the events are selected for the structure function analysis to ensure good efficiency and small acceptance corrections. The selection criteria is divided into fiducial-volume cuts and geometric cuts. 


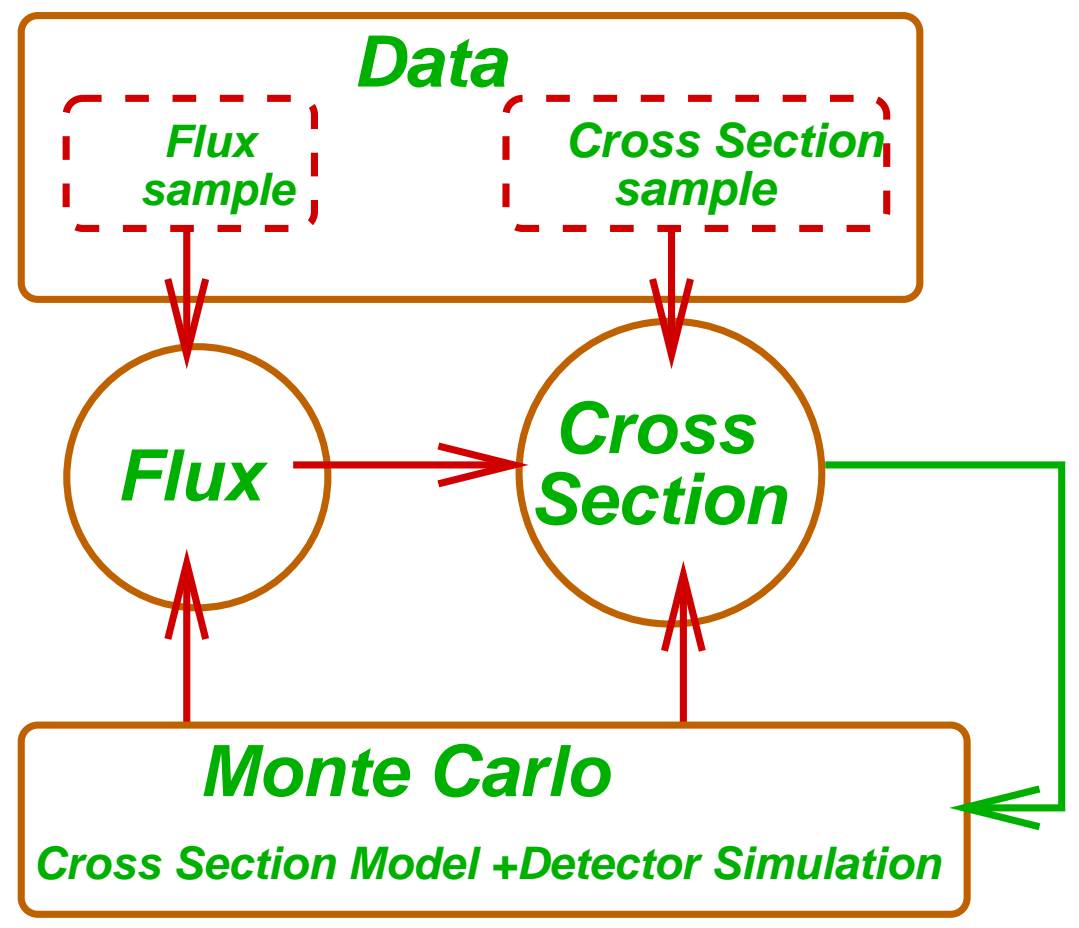

Figure 3.1: A diagram of the cross-section extraction mechanism.

\subsubsection{Fiducial-Volume Cuts}

The fiducial volume cuts assure that the event takes place within the active area of the detector. These are:

- BADRUNS: removes runs or events for which there are known detector hardware failures.

- IGATE: selects neutrino run of the accelerator cycle (fast gate).

- NSTIME: selects single interaction events (muon track time required to be within 36 ns of the hadron shower).

- $20<$ PLACE $<$ 80: assures good longitudinal containment of the hadron shower in the calorimeter.

- $\left|\mathbf{V}_{\mathbf{x}}\right|<\mathbf{5 0}$ in, $\left|\mathbf{V}_{\mathbf{y}}\right|<\mathbf{5 0}$ in: assures good transverse containment of hadron shower in 
the calorimeter.

\subsubsection{Geometric Cuts}

Geometric cuts assure good acceptance of the detector and quality of the muon track. The following cuts are considered:

- TRIGGER 1: selects the charged current events with muon track in the calorimeter and hits in the toroid drift chambers.

- TARGET TRACK: requires hits in the target drift chambers for a good identification of a muon track. This is important for an accurate muon angle determination.

- TOROID CONTAINMENT: the extrapolated muon track from hits in the target to toroid is required to have a radius at the front face of the toroid $\mathrm{R}_{\mathrm{FF}}<60 \mathrm{in}$, and at T2 the radius to be $R_{\mathrm{T}_{2}}<55$ in. This cut assures good muon track in the toroid.

- TOROID TRACK: requires hits in the toroid drift chambers for a good muon track reconstruction in the toroid.

- HOLE CUT: requires $\theta_{\mu}>0.007 \mathrm{rad}$ and $\mathrm{R}_{\mathrm{FF}}>6$ in to assure muon not passing through the hole in the center of the spectrometer where magnetic field is not well modeled.

- 80\%: of toroid muon track within the steel of the toroid is required.

- GOOD FIT: requires a fitted muon track in the toroid, with number of degrees of freedom corresponding to numbers of drift chambers with hits.

- TWO GAPS: requires hits in the both first and second gaps drift chambers to ensure a good determination of muon momentum. 


\subsubsection{Data Samples}

Data is divided into nearly two independent data samples used for cross-section analysis (cross section sample) and flux extraction (flux sample) as shown in Figure 3.1.

\subsubsection{Flux sample}

This sample corresponds to data of low hadronic energy used to determine the relative neutrino flux (described in Sect. 3.3). It requires in addition to fiducial volume and geometric cuts the following cuts:

- $\mathbf{E}_{\text {had }}<20 \mathrm{GeV}$ : to allow the use of "fixed- $\nu_{0}$ " method to extract relative flux as discussed in Sect. 3.3.

- $\theta_{\mu}<\mathbf{1 5 0}$ mrad: to ensure good acceptance of the muon track in the toroid.

- $600>\mathbf{E}_{\mu}>\mathbf{1 5} \mathrm{GeV}$ and $\mathbf{E}_{\mu}^{\mathbf{F F}}>\mathbf{3} \mathrm{GeV}$ : Very high energy muons have straight tracks in muon spectrometer resulting in poor momentum resolution. Low energy muons are poorly reconstructed due to multiple Coulomb scattering.

Total number of events passing the flux cuts was found to be $4.3 \times 10^{5}$ for neutrinos and $2.1 \times 10^{5}$ for antineutrinos [22].

\subsubsection{Cross section sample}

This sample is used to extract the DIS neutrino differential cross section on Iron. In addition to fiducial volume and geometric cuts the following cuts are required:

- $\theta_{\mu}<150 \mathrm{mrad}, 600>\mathbf{E}_{\mu}>15 \mathrm{GeV}$ and $\mathbf{E}_{\mu}^{\mathrm{FF}}>3 \mathrm{GeV}$ (the same as for the flux sample).

- $600>\mathbf{E}_{\text {had }}>\mathbf{1 0} \mathrm{GeV}$ - The low hadron energy cut minimizes the correlation with the flux sample, and removes quasi-elastic and resonances effects. 
- $\mathrm{Q}^{2}>\mathbf{1} \mathrm{GeV}^{2}$-This cut minimizes the non-perturbative contributions, and reduces the contributions from "quasi-elastic" events.

- $360>\mathbf{E}_{\nu}>\mathbf{3 0} \mathrm{GeV}$ - This cut ensures that the flux is well understood and the muon momentum is well reconstructed.

There were $8.7 \times 10^{5}$ neutrino and $2.4 \times 10^{5}$ antineutrino events passing the cuts for cross section analysis [22]. Table 3.1 summarizes the number of events in each data sample after all cuts were applied.

Table 3.1: Number of events in each data sample.

\begin{tabular}{|l|l|l|}
\hline \hline Mode & Cross Section Sample & Flux Sample \\
\hline$\nu$ & 866,000 & 426,803 \\
$\bar{\nu}$ & 240,000 & 210,795 \\
\hline \hline
\end{tabular}

\subsection{MONTE CARLO SIMULATION}

The only purpose of a Monte Carlo Simulation in the extraction of neutrino differential cross section analysis is to correct data samples for acceptance and smearing effects. For this, the $\mathrm{NuTeV}$ analysis uses a detailed Monte Carlo event simulation program called NUMONTE. The NUMONTE program provides a full tracking for high energy muons, but relies on the parametrization of the detector response from calibration beam data for other final state particles (e.g. hadron showers). Therefore, this Monte Carlo is fast, as it can simulate several million neutrino events in a matter of hours. Since cosmic rays and beam muons are removed from data through a stringent set of cuts, there is no need to model them. 
The program consists of three main building blocks: cross section model, event generation of a neutrino interaction, and detector model to simulate the response of the detector for each generated event. Each of these blocks are discussed briefly in the following Sections.

\subsubsection{Cross-Section Model}

A neutrino cross-section model is used in extraction of the neutrino flux from the low-hadron energy data (described in Sect.3.3), in determination of the detector acceptance and resolution effects, and in physics corrections such as radiative corrections, isoscalarity, propagator corrections, charm production thresholds. Therefore, NUMONTE requires as input a model for neutrino cross-section which is iteratively fit to our data. The neutrino cross-section model in NUMONTE is based on the standard deep inelastic formalism which was described in Section 1.2. We assume the V-A structure of the neutrino cross section, and the chargedcurrent neutrino-nucleon DIS cross-section is expressed in terms of three structure functions as in Eq. (1.27). The NuTeV analysis uses an enhanced LO QCD A. J. Buras - K. F.J. Gaemers model [38] with added terms to allow deviations from a strict LO calculation. This model is intended to be a simple phenomenological fit to our data (in order to reduce the theory dependencies which would otherwise contribute large uncertainties) and should not depend on specifics of QCD.

In an enhanced LO model, the structure functions can be expressed in terms of PDFs as

$$
\begin{aligned}
2 x F_{1}\left(x, Q^{2}\right) & =x u_{v}+x d_{v}+2 x u_{s}+2 x d_{s}+2 x s, \\
F_{2}\left(x, Q^{2}\right) & =2 x F_{1}\left(x, Q^{2}\right)\left(\frac{1+R_{L}\left(x, Q^{2}\right)}{1+4 M^{2} x^{2} / Q^{2}}\right), \\
x F_{3}\left(x, Q^{2}\right) & =x u_{v}+x d_{v},
\end{aligned}
$$

where the PDFs are for an iron target. Thus, the cross section model can be parametrized in terms of these PDFs. 


\subsubsection{Parametrization}

The PDF parametrization can be separated into valence quark $\left(u_{v}, d_{v}\right)$, light quark sea $(\bar{u}, \bar{d})$, strange sea distributions $(s, \bar{s})$, charm sea $(c, \bar{c})$ (neglected in this model), and gluons. The parametrization is performed as function of Bjorken- $x$, at a starting value of $Q_{0}^{2}=12.6 \mathrm{GeV}^{2}$ and then evolved to any $Q^{2}$ using functional forms similar to QCD, but without imposing exact QCD calculations.

\section{- valence distributions:}

The parametrization is characterized with a functional form as

$$
\begin{aligned}
& x u_{v}\left(x, Q_{0}^{2}\right)=u_{v}^{t o t}\left[x^{E_{1}}(1-x)^{E_{2}}+A V_{2} x^{E_{3}}(1-x)^{E_{4}}\right] \\
& x d_{v}\left(x, Q_{0}^{2}\right)=d_{v}^{t o t} x u_{v}\left(x, Q_{0}^{2}\right)(1-x)
\end{aligned}
$$

where exponents $E_{1, \ldots, 4}$ and parameter $A V_{2}$ describe $u$ and $d$ valence distribution, where the $d$ valence distribution is connected to the $u$ valence distribution by the $(1-x)$ factor (neutrino scattering is relatively insensitive to the difference between $u_{v}$ and $d_{v}$ ). Parameters $u_{v}^{\text {tot }}$ and $d_{v}^{\text {tot }}$ are the overall normalizations for the valence distributions. The evolution of the valence distributions is performed according to a simple logarithmic function (proposed by Buras and Gaemers, [38]):

$$
\begin{aligned}
& E_{1}=E_{10}+s E_{11}, \\
& E_{2}=E_{20}+s E_{21}, \\
& E_{3}=E_{30}+s E_{21}, \\
& E_{4}=E_{40}+s E_{21},
\end{aligned}
$$

where,

$$
s=\log \frac{\log \left(\frac{Q^{2}}{A_{0}^{2}}\right)}{\log \left(\frac{Q_{0}^{2}}{A_{0}^{2}}\right)} .
$$


The exponents $E_{10, \ldots, 40}$ correspond to parametrization of the valence distributions independent of $Q^{2}$. The parameter $A_{0}^{2}$ takes the place of $\Lambda_{Q C D}$ in QCD. Valence normalization parameters are constrained by the corrected Fermion Sum Rule (GLS) in powers of $\alpha_{S}$ as

$$
\int_{0}^{1} F_{3}\left(x, Q^{2}\right) d x=3\left(1-\frac{\alpha_{S}\left(Q^{2}\right)}{\pi}\right)=3\left(1-\frac{A_{1}}{\log \frac{Q^{2}}{A_{0}^{2}}}-\frac{A_{2}}{\left(\log \frac{Q^{2}}{A_{0}^{2}}\right)^{2}}\right)
$$

and by quark charge constraint as

$$
\left(+\frac{2}{3}\right) \int_{0}^{1} u_{v}\left(x, Q^{2}\right) d x+\left(-\frac{1}{3}\right) \int_{0}^{1} d_{v}\left(x, Q^{2}\right) d x=1-\frac{A_{1}}{\log \frac{Q^{2}}{A_{0}^{2}}}-\frac{A_{2}}{\left(\log \frac{Q^{2}}{A_{0}^{2}}\right)^{2}}
$$

Note that although the parameters are constraint by QCD-like functional forms, their values are allowed to float (in order not to bias our results).

\section{- light sea distributions:}

The light sea quark distributions, $\bar{u}, \bar{d}$, are assumed to have the same behavior in $x$ and $Q^{2}$. Their parametrization is given by

$$
\begin{aligned}
x \bar{u}\left(x, Q^{2}\right) & =x \bar{d}\left(x, Q^{2}\right)=\frac{1}{2(k+2)} x S\left(x, Q^{2}\right) \\
& =\frac{1}{2(k+2)}\left(A S(1-x)^{E S}+A S_{2}(1-x)^{E S_{2}}\right),
\end{aligned}
$$

where $k$ relates the normalization of the strange quark sea to the non-strange quark sea. The light quark sea distributions decrease rapidly with $x$, therefore can be be determined from the first two moments as

$$
\begin{aligned}
& S_{2}=\int_{0}^{1} x S d x=\frac{3}{4} D_{22}+\frac{1}{4} D_{12} \\
& S_{3}=\int_{0}^{1} x^{2} S d x=\frac{3}{4} D_{23}+\frac{1}{4} D_{13}
\end{aligned}
$$


where $D_{i j}$ are completely specified by LO QCD [38]. The parameters $A S_{2}, E S_{2}$ are evolved in $Q^{2}$ according to

$$
\begin{aligned}
& A S_{2}=A S_{20}+A S_{21} \log Q^{2}, \\
& E S_{2}=E S_{20}+E S_{21} \log Q^{2},
\end{aligned}
$$

and $E S, A S$ depend on second and third moments of the light sea distributions.

\section{- strange sea distributions:}

The $s$ and $\bar{s}$ quark distributions are important in neutrino scattering because when a neutrino strikes a $s$ or $\bar{s}$ quark it produces a heavy charm quark, which decays into a high-energy muon (dimuon event). Therefore, the strange sea is constrained by the LO fit to $\mathrm{CCFR} / \mathrm{NuTeV}$ dimuon differential cross section data [39] ${ }^{1}$. This model assumes strange sea asymmetry, but it allows a different shape from the light quark distributions:

$$
\begin{aligned}
x s\left(x, Q^{2}\right) & =x \bar{s}\left(x, Q^{2}\right) \\
& =\frac{k}{2(k+2)} \frac{A S}{E S+1}(E S+1+\alpha)(1-x)^{E S+\alpha},
\end{aligned}
$$

where $\alpha$ describes the relative shapes between the strange and non-strange sea (i.e., light quark sea).

- Charm sea distribution: is neglected in this model.

- Gluon distribution: enters in the model only through missing quarks terms.

There are 19 free PDF fit parameters:

$A V_{2}, A_{0}, A_{1}, A_{2}, E_{10}, E_{20}, E_{21}, E_{30}, E_{40}, S_{2}, S_{3}, A S_{20}, A S_{21}, E S_{20}, E S_{21}, A_{1}, A_{2}, G_{0}$.

\footnotetext{
${ }^{1}$ New NLO NuTeV measurement of the strange sea from dimuon data was performed, see Ref. [26]
} 


\subsubsection{Physical Corrections}

This parametrization of the cross section model needs to be adjusted to account for various physical effects not included in the LO model. The following corrections were added:

- $\mathbf{d} / \mathbf{u}$ Correction. PDF parametrization assumes up and down seas the same. However, an asymmetry has been observed by Drell-Yan data [40]. The form of this asymmetry was parametrized as following:

$$
f(\bar{d} / \bar{u})=\frac{1}{M A X\left(1-x\left(2.7-0.14 \log Q^{2}-1.9 x\right), 0 / 1\right)}
$$

Thus, the light sea quark distributions corrected for this asymmetry can be written as

$$
\begin{aligned}
\overline{u^{\prime}} & =\bar{u} \frac{\bar{u}+\bar{d}}{\bar{u}+f(\bar{d} / \bar{u}) \bar{d}} \\
\overline{d^{\prime}} & =\bar{d} \frac{\bar{u}+\bar{d}}{\bar{u}+f(\bar{d} / \bar{u}) \bar{d}} f(\bar{d} / \bar{u})
\end{aligned}
$$

with the constraint that $\bar{u}+\bar{d}=\overline{u^{\prime}}+\overline{d^{\prime}}$.

Valence distributions $\left(d_{v}\right.$ and $\left.u_{v}\right)$ also are corrected using parametrization of $F_{2}^{d} / F_{2}^{p} \mathrm{NMC}$ data [18], so that

$$
\delta(d / u)=0.12079-1.3303 x+4.9829 x^{2}-8.4465 x^{3}+5.7324 x^{4},
$$

which lead to the following correction for valence distributions:

$$
\begin{aligned}
u_{v}^{\prime} & =\frac{u_{v}}{1+\delta(d / u) \frac{u_{v}}{u_{v}+d_{v}}} \\
d_{v}^{\prime} & =\frac{d_{v}+u_{v} \delta(d / u)}{1+\delta(d / u) \frac{u_{v}}{u_{v}+d_{v}}}
\end{aligned}
$$


- Higher-Twist Correction. This correction is used to account for non-perturbative QCD effects which are important at high- $x$ and low $Q^{2}$ region. It is determined from fits to $F_{2}$ charged lepton data (proton and deuterium) $[43,45,44]$ for $x>0.4$ using an empirical model [42], which rescales $x$ to include a $Q^{2}$ dependence such as,

$$
x \rightarrow \xi_{H T}=x \frac{Q^{2}+B_{H T}}{Q^{2}+x A_{H T}},
$$

where $A_{H T}, B_{H T}$ enter as fit parameters in the cross section model. Before the fit is performed, charged lepton data needs to be converted to neutrino-iron data. The following corrections have to be applied:

1. Correction for the electric charge assignment to quarks for electromagnetic interaction versus weak interaction. This correction is referred to as the " $5 / 18$ th" rule:

$$
F_{2}^{l d}\left(x, Q^{2}\right)=F_{2}^{\nu d}\left(x, Q^{2}\right) \frac{5}{18}\left(1-\frac{3}{5} \frac{x s+x \bar{s}}{x d_{v}+x u_{v}+2 x d_{s}+2 x u_{s}+2 x s}\right)
$$

where $F_{2}^{l d}$ is the deuteron structure function $F_{2}$ for charged lepton data and $F_{2}^{\nu d}$ is for neutrino data. This relation is valid at all orders of perturbation theory if it is defined in the DIS factorization scheme. Therefore, the CTEQ4D scheme (where D stands for DIS scheme) is used for the PDFs.

2. Correction for the difference in light versus heavy nuclear targets. This correction is given by a parametrization to the charged lepton data (described in Sect.1.3.4.3) of the form

$$
f(x)=1.10-0.36 x-0.28 e^{-21.9 x}+2.77 x^{14.4} .
$$


The charged lepton data is included in the PDF fit in order to constrain better the fit at the edge of our data acceptance. The relative normalizations among each data sets are allowed to float. The $\chi^{2}$ for each data set is calculated separately and then added to the fit of the $\mathrm{NuTeV}$ data to extract the parameters of the cross-section model. Figure 3.2 shows the effect of including the higher twist parametrization in the cross-section model (before and after) compared to SLAC and BCDMS $F_{2}\left(x, Q^{2}\right)$ data $[43,44]$ at high $x$ and low $Q^{2}$. The solid curve in the Figure corresponds to our model.

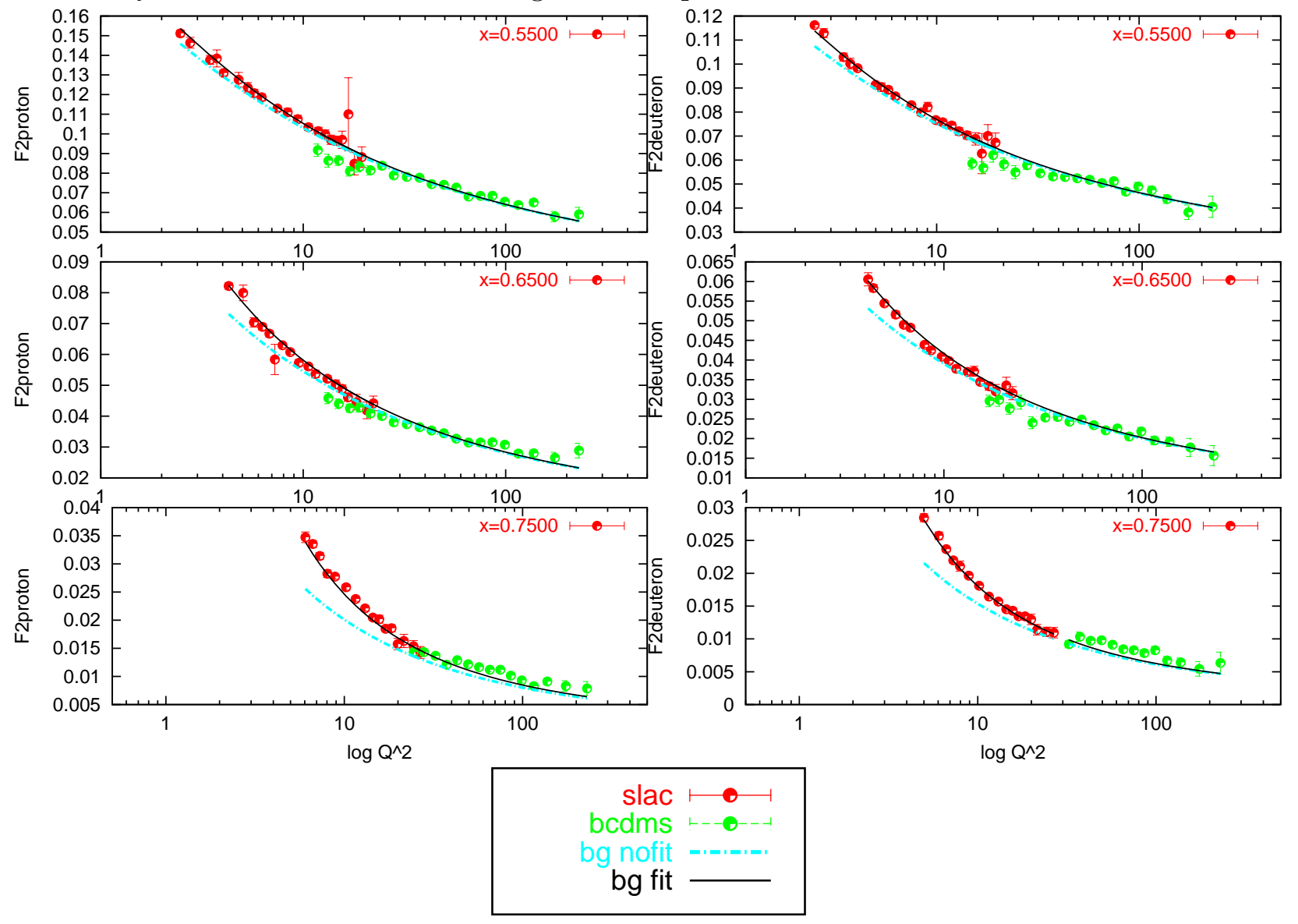

Figure 3.2: The effects of applying higher-twist effects on $\mathrm{NuTeV}$ cross section model compared on SLAC and BCDMS $F_{2}\left(x, Q^{2}\right)$ proton and deuteron data.

- Low- $Q^{2}$ extrapolation. At low $x$ and $Q^{2}$ the Buras-Gaemers parametrization is not well behaved. Therefore, the $Q^{2}$ shape of GRV94LO PDFs [41] is used to extrapolate 
this model down to $Q^{2}=0.23 \mathrm{GeV}^{2}$ with matching performed at $Q^{2}=1.35 \mathrm{GeV}^{2}$. The extrapolation is used in order to model well the edge at low $x$ and $Q^{2}$ of our data range.

- Longitudinal Structure Function $R_{L}$. It is introduced in the Monte Carlo Simulation in order to account for gluon effects in the cross section model. As a consequence, the Callan-Gross relation 1.36 is violated, such that

$$
F_{2}\left(x, Q^{2}\right)=\frac{1+R_{L}\left(x, Q^{2}\right)}{1+\frac{4 M^{2} x^{2}}{Q^{2}}} 2 x F_{1}\left(x, Q^{2}\right)
$$

For the structure function $R_{L}$ an empirical parametrization of the world's available data on $R_{L}$ is used [46]:

$$
R_{L}\left(x, Q^{2}\right)=\frac{0.0635}{\log \frac{Q^{2}}{0.04}} \Theta\left(x, Q^{2}\right)+\frac{0.5747}{Q^{2}}-\frac{0.3534}{Q^{4}+0.09}
$$

where $\Theta\left(x, Q^{2}\right)$ is

$$
\Theta\left(x, Q^{2}\right)=1+12\left(\frac{Q^{2}}{1+Q^{2}}\right)\left(\frac{0.125^{2}}{x^{2}+0.125^{2}}\right) .
$$

- Non-Isoscalar Target corrections. The NuTeV target is made of iron which has an excess of neutrons compared to protons (non-isoscalar target) by

$$
\frac{N-Z}{A}=0.0567
$$

where $N, Z, A$ are the number of neutrons, protons, nucleons, respectively. This correction is taken into account in NUMONTE by computing separately the differential cross section for protons and neutrons. Then, a weighted average is computed as

$$
\frac{d^{2} \sigma^{\nu(\bar{\nu}) N}}{d x d y}=\frac{Z}{A} \frac{d^{2} \sigma^{\nu(\bar{\nu}) p}}{d x d y}+\frac{N}{A} \frac{d^{2} \sigma^{\nu(\bar{\nu}) n}}{d x d y}
$$



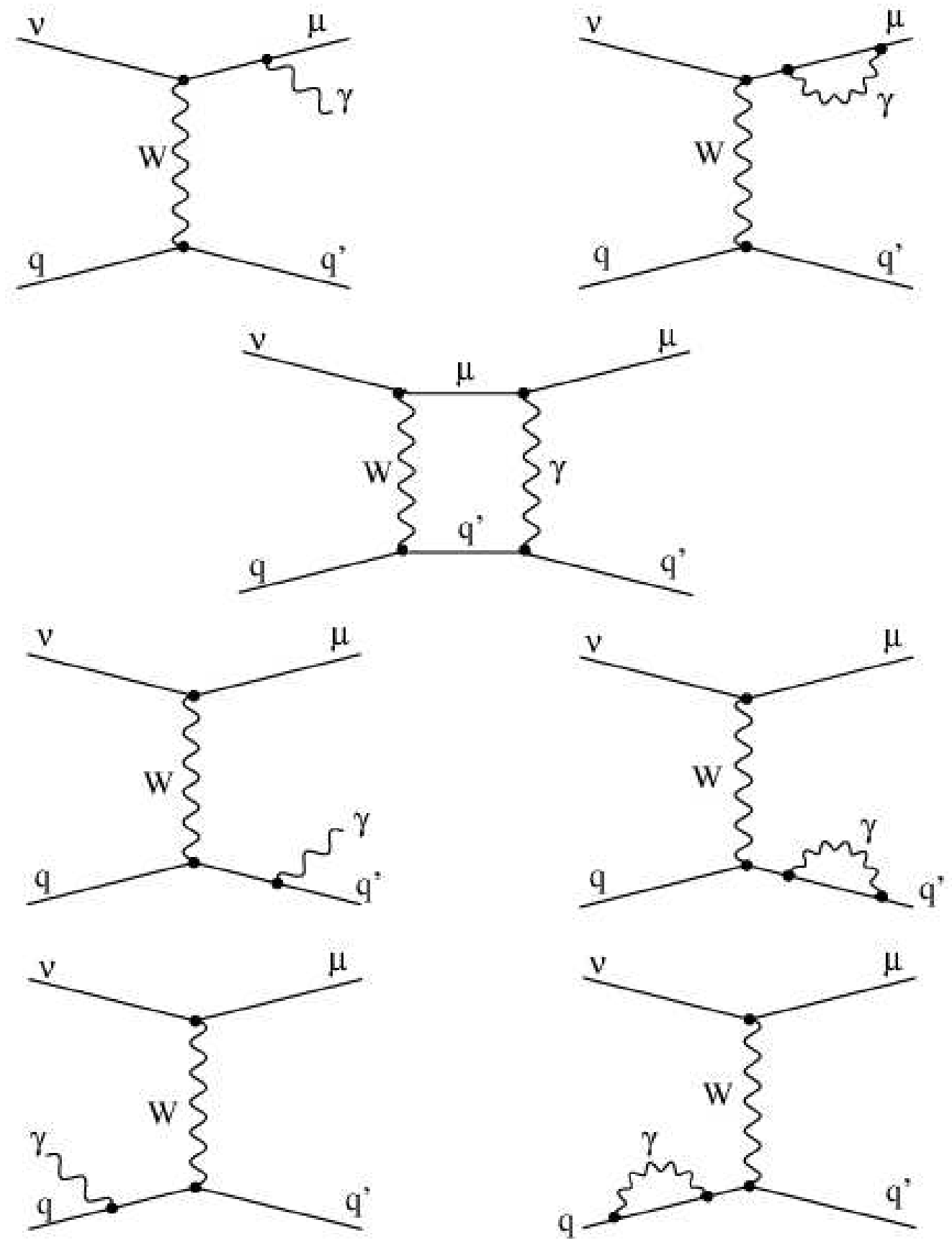

Figure 3.3: Feynman diagrams for QED radiative corrections for charged-current neutrino scattering. 
- Radiative Corrections account for radiation of real and virtual photon by the charged particles. Figure 3.3 shows some of the Feynman diagrams for QED radiative corrections for CC neutrino scattering. The correction is computed by Bardin [47] within the framework of Quark Parton Model and stored in a table in $x,, y, E$ bins. This correction is applied to bare cross section as

$$
\frac{d^{2} \sigma^{\nu(\bar{\nu})}}{d x d y} \rightarrow\left(\frac{\left(\frac{d^{2} \sigma^{\nu(\bar{\nu})}}{d x d y}\right)_{Q E D-1-\text { loop }}}{\left(\frac{d^{2} \sigma^{\nu(\bar{\nu})}}{d x d y}\right)_{\text {bare }}}\right)\left(\frac{d^{2} \sigma^{\nu(\bar{\nu})}}{d x d y}\right)_{\text {bare }} .
$$

- Propagator Term. The correction for the massive mediating $W$ boson of a neutrino interaction is taken into account as follows:

$$
\frac{d^{2} \sigma^{\nu(\bar{\nu})}}{d x d y} \rightarrow \frac{1}{\left(1+\frac{Q^{2}}{M_{W}^{2}}\right)^{2}} \frac{d^{2} \sigma^{\nu(\bar{\nu})}}{d x d y} .
$$

- Charm Production Threshold. About $10 \%$ of total charged current events produce heavy charm quarks when a neutrino strikes off a $d$ or $s$ quark. The model uses a slow rescaling method to account for charm production in neutrino scattering $[15,49]$. This method rescales Bjorken- $x$ taking into account charm mass $m_{c}$ such as

$$
x \rightarrow \xi=x\left(1+\frac{m_{c}^{2}}{Q^{2}}\right) .
$$

The heavy quark thresholds are accounted in the model by applying the following modifications to the structure functions:

$$
\begin{aligned}
2 x F_{1}\left(x, Q^{2}\right) & \rightarrow \frac{x}{\xi} 2 \xi F_{1}\left(\xi, Q^{2}\right) \\
x F_{3}\left(x, Q^{2}\right) & \rightarrow \frac{x}{\xi} \xi F_{3}(\xi) \\
F_{2}\left(x, Q^{2}\right) & =\frac{1+R_{L}\left(\xi, Q^{2}\right)}{1+\frac{4 M^{2} \xi^{2}}{Q^{2}}} 2 \xi F_{1}\left(\xi, Q^{2}\right) .
\end{aligned}
$$

These adjustments depend on the charm mass parameter, its value is obtained from the weighted average of leading-order measurements, $m_{c}=1.4 \pm 0.18 \mathrm{GeV}[39,48]$. This value is used through out this thesis. 


\subsubsection{Cross Section Fit Results}

The PDFs are determined from the fit to the extracted cross section tables (described in Sect. 3.4) using the above cross section model. The $\chi^{2}$ of the fit is constructed as follows:

$$
\chi^{2}=\sum_{i=1}^{N^{\text {data }}} \frac{\left(X_{i}^{\text {DATA }}-X_{i}^{\text {model }}\right)^{2}}{\sigma_{i}^{2}}
$$

where $X_{i}^{D A T A}$ are the extracted $\mathrm{NuTeV}$ cross section data points and $X_{i}^{\text {model }}$ corresponds to the cross section model parametrized through 21 parameters (including the higher twist parameters). The charged lepton data is included in this fit in order to constrain better cross section model as described in Sect. 3.2.1.2:

$$
\chi^{2}=\chi_{N u T e V}^{2}+\chi_{S L A C}^{2}+\chi_{N M C}^{2}+\chi_{B C D M S}^{2}
$$

The fit is performed for the kinematic region of $0.001<x<0.7,30<E_{\nu}<360(\mathrm{GeV})$, and $Q^{2}>1.35\left(\mathrm{GeV}^{2}\right)$. Note that the fit is performed iteratively and the final fit results are presented in the Table 3.2 [22]. The iteration procedure is outlined in Sect.3.4.

\subsubsection{Neutrino Event Generation}

The probability of having a neutrino interaction in the detector is proportional to the flux of neutrino passing through the detector times the cross section of that interaction:

$$
\mathrm{P}\left(E_{\nu}, V_{x}, V_{y}, x, y\right) \propto \Phi\left(E_{\nu}, V_{x}, V_{y}\right) \frac{d^{2} \sigma\left(E_{\nu}, x, y\right)}{d x d y}
$$

NUMONTE generates charged current neutrino events for a given cross section model (described above) and a neutrino flux (determined from low hadron energy data sample, as discussed in Sect. 3.3).

The event generation proceeds through the following steps ${ }^{2}$ :

\footnotetext{
${ }^{2}$ NUMONTE assigns to the "TRUE" generated variables a "G" ending to their names to distinguish them from smeared variables.
} 
Table 3.2: Fit results for $\mathrm{NuTeV}$ cross section model after final iteration.

\begin{tabular}{|c|c|}
\hline \hline Parameters & Value \pm Error \\
\hline$A_{0}$ & $0.583 \pm 0.017$ \\
$A_{1}$ & $0.295 \pm 0.013$ \\
$A_{2}$ & $0.17 \pm 0.03$ \\
$A V_{2}$ & $637 \pm 75$ \\
$E_{10}$ & $0.5333 \pm 0.0025$ \\
$E_{11}$ & $-0.028 \pm 0.011$ \\
$E_{20}$ & $2.61 \pm 0.015$ \\
$E_{21}$ & $1.31 \pm 0.045$ \\
$E_{30}$ & $4.56 \pm 0.14$ \\
$E_{40}$ & $12.5 \pm 0.35$ \\
$S_{2}$ & $0.1625 \pm 0.0013$ \\
$S_{3}$ & $0.01589 \pm 0.0004$ \\
$G_{3}$ & $0.031 \pm 0.003$ \\
$A S_{20}$ & $1.06 \pm 0.11$ \\
$A S_{21}$ & $1.76 \pm 0.25$ \\
$E S_{20}$ & $185 \pm 20$ \\
$E S_{21}$ & $8.4 \pm 8.0$ \\
$A_{H T}$ & $1.187 \pm 0.035$ \\
$B_{H T}$ & $0.3332 \pm 0.02$ \\
\hline \hline
\end{tabular}

- The neutrino type (neutrino or antineutrino mode) and toroid polarity is selected.

- Neutrino energy $(E N U G)$ and vertex coordinates transverse to the beam axis $(V X G$, 
$V Y G$ ) are randomly selected from measured flux distribution (as discussed in Sect. 3.3).

- PLACE, the longitudinal interaction position, is randomly thrown from a uniform distribution.

- The Bjorken- $X G$ and inelasticity $Y G$ are thrown according to the cross section model.

- The other kinematic variables like muon momentum $(E M U G)$, hadronic energy $(E H A D G)$, muon angle (THETAMUG) can be calculated from the above generated distributions as in Eq. (1.5-1.11).

- The hadron calibration beam is used to determine the dependence of the shower distribution on hadron energy. This library of hadron showers is used to generate the longitudinal location of the shower end $(S H E N D)$. Thus, the hadron shower length, defined as $P L A C E-S H E N D+5$ (as it is defined for data), is generated.

- The tracking of the muon through the target is performed by stepping through each counter unit and computing energy loss (simulating MCS for each step). The muon energy loss $(D E D X G)$ is generated in two parts: restricted (due to ionization) and catastrophic losses (due to Bremsstrahlung, pair production, delta ray production). Range tables are used for small energy losses $(<1 \mathrm{GeV})$. Therefore, the generated muon energy at the front face of the toroid $(E M U F F G)$ is obtained by subtracting $D E D X G$ from the generated muon energy at the vertex $(E M U G), E M U F F G=E M U G-D E D X G$.

\subsubsection{Detector Model}

To simulate the detector response, NUMONTE smears the generated kinematic variables using either a parametrization from calibration beam data or a simulated response by GEANT hit-level Monte Carlo [36]. The same procedure as in data is used to reconstruct the variables. Bellow we sketch the procedure to smear the main generated kinematic quantities used in NuTeV analysis (see Ref. [22] for more details). 


\section{- Muon Energy smearing and reconstruction:}

The total muon energy is reconstructed in three pieces: muon energy in the toroid, in the target calorimeter beyond the shower region, and within the shower region.

The generated toroid muon energy is smeared by sampling the toroid resolution function, given by

$$
R=\frac{1 / P_{\mu}^{F F}(\text { meas })-1 / P_{\mu}^{F F}(\text { gen })}{1 / P_{\mu}^{F F}(\text { gen })},
$$

where $P_{\mu}^{F F}$ (meas) and $P_{\mu}^{F F}($ gen $)$ are the smeared and generated muon momenta in the toroid. The muon momentum resolution function is displayed in Figure 2.24.

The restricted energy loss is smeared with a Gauss distribution of width $0.045 \mathrm{GeV}$. The catastrophic energy loss is instead smeared using calibration data for electrons with a Gaussian smearing function of width $0.5 \sqrt{E_{\text {catastrophic }}}$.

NUMONTE simulates the pulse height(in MIP) for each counter using the conversion factors determined from the calibration beams (see Sect. 2.5). The restricted energy loss is converted to MIPs using $C_{\mu}$ conversion factor, while the catastrophic energy loss uses the electromagnetic conversion factor, $C_{e}$. The simulated pulse height in each counter is given by the sum of catastrophic and restricted energies loss per each counter in MIPs. Therefore, the reconstructed muon energy in the target beyond the shower region is determined by summing up the pulseheights from SHEND-6 to 1 and converting them into GeV using Eq. (2.18). NUMONTE uses the same parametrization of the muon energy loss in the shower region as it is used for the real data.

\section{- Hadron Energy Smearing:}

The generated hadron energy is corrected for the observed non-linearity in the NuTeV calorimeter, then it is smeared by sampling the Poisson-like resolution function given by Eq. (2.15). The contribution of the muon energy deposited in the shower region was simulated by adding to the smeared hadron energy the simulated pulse-heights in 
the counters within the shower region. The muon energy loss, which is parametrized according to the restricted energy loss, is subtracted from the hadronic energy.

\section{- Muon Angle Smearing:}

As described in Sect. 2.5.4, angular resolution is dominated by MCS effects in the target and is parametrized using GEANT hit-level simulation.

$$
\Delta \theta_{\mu}=(96.8+0.87 P L A C E+0.24 E H A D G) \frac{1}{P_{\mu}^{G}}
$$

where $P_{\mu}^{G}=\sqrt{(E M U G)^{2}-m_{\mu}^{2}}$ is the generated muon momentum.

From the smeared generated variables presented above one ca obtain the remaining kinematic quantities using Eqs. (1.5-1.11).

Figures 3.4 and 3.5 show data distributions for muon energy, hadron energy, and muon angle at the vertex compared to Monte Carlo simulation(for neutrino and antineutrino modes). NUMONTE models real data very well, at the level of $2 \%$.

\subsection{NEUTRINO FLUX EXTRACTION}

Obviously, the neutrino flux cannot be determined directly, while monitoring the secondary meson beam for this experiment was too intense to allow a precise measurement of the neutrino flux. Therefore, $\mathrm{NuTeV}$ measures the neutrino flux from the number of charged current interactions in the detector using a low hadronic data sample. This method is referred to as "fixed- $\nu_{0}$ " method $[50,33]$ (summarized below) and it actually determines the relative flux in energy bins. The overall normalization is determined using the world average of absolute cross section measurements. 

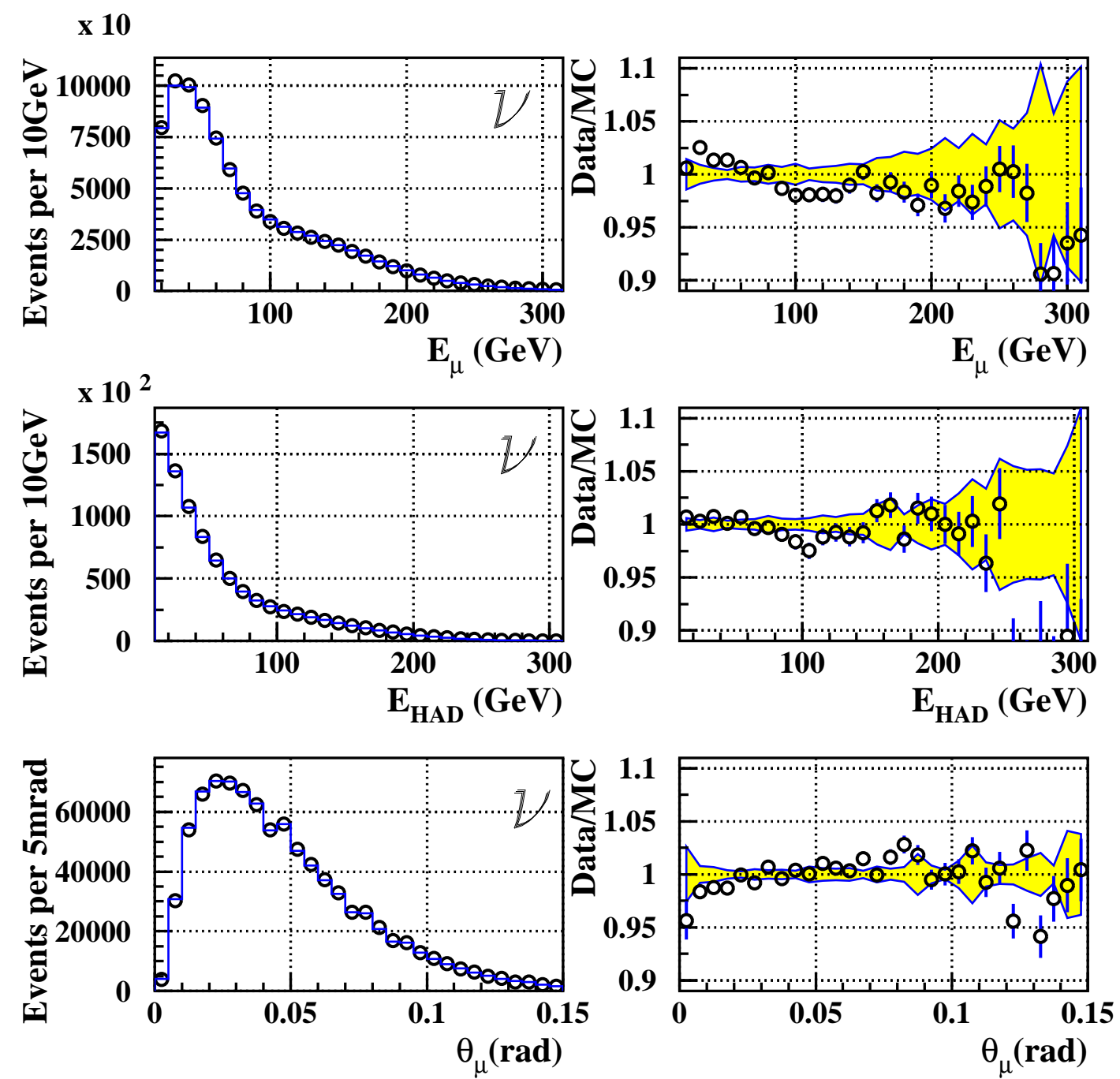

Figure 3.4: Data - Monte Carlo comparison for muon energy, hadron energy and muon angle (neutrino mode). Systematic uncertainty is shown with yellow band. Plots are courtesy of M. Tzanov. 

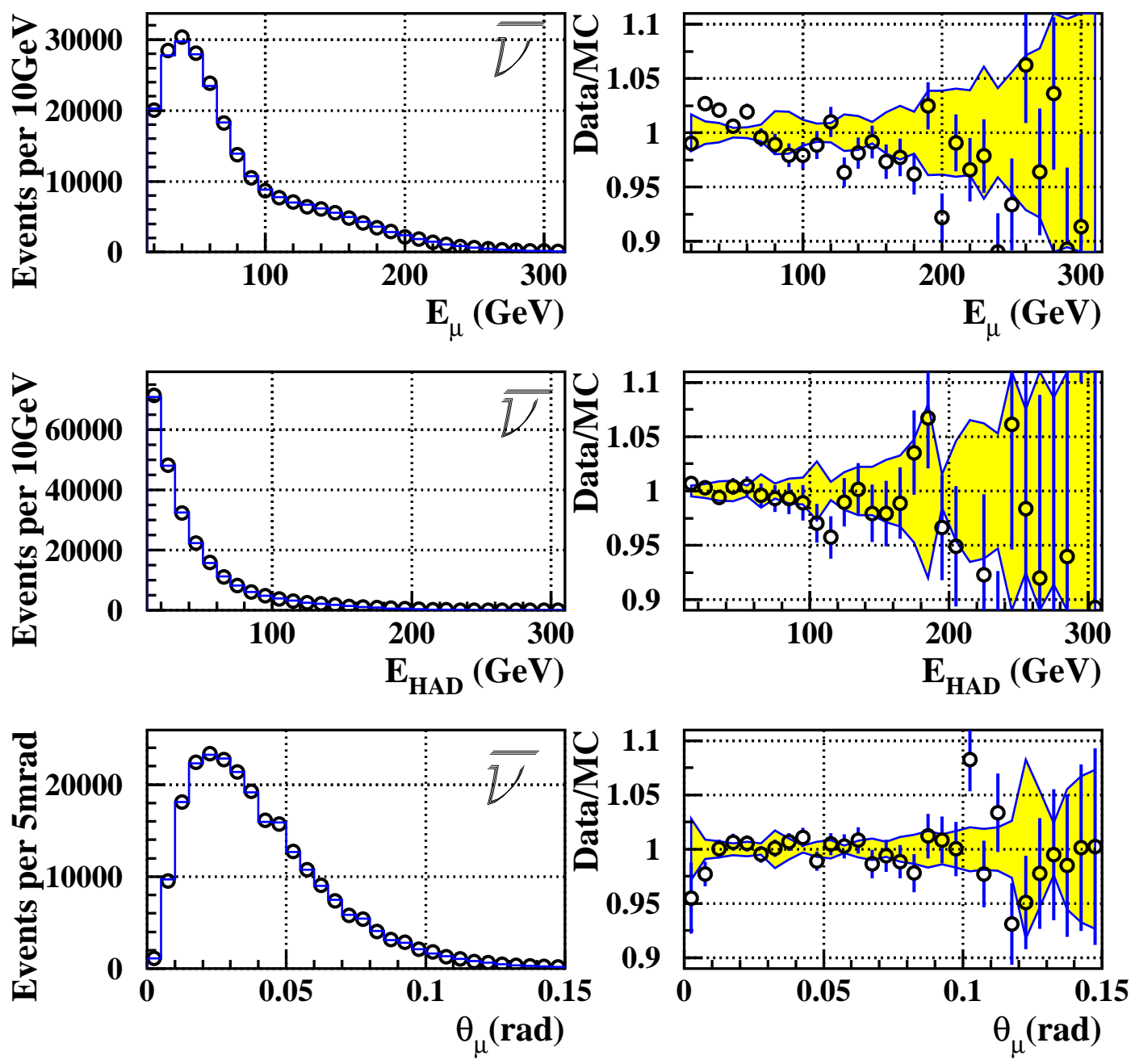

Figure 3.5: Data - Monte Carlo comparison for muon energy, hadron energy and muon angle (antineutrino mode). Systematic uncertainty is shown with yellow band. Plots are courtesy of M. Tzanov. 


\subsubsection{Fixed $E_{\text {had }}$ Method}

The relative flux is determined from flux data sample of low hadronic energy (sample defined in Sect.3.1.3.1). DIS cross section from Eq. (1.31) can be re-written in terms of $\nu \equiv E_{\text {had }}$ instead of inelasticity $y$ using $\nu=E_{\nu} y=E_{\text {had }}$ as:

$$
\frac{d^{2} \sigma^{\nu(\bar{\nu})}}{d x d y}=\frac{G^{2} M}{\pi}\left[\left(1-\frac{\nu}{E_{\nu}}-\frac{M x \nu}{2 E_{\nu}^{2}}+\frac{\nu^{2}}{2 E_{\nu}^{2}} \frac{1+\frac{2 M}{\nu}}{1+R_{L}} F_{2} \pm \frac{\nu}{E_{\nu}}\left(1-\frac{\nu}{2 E_{\nu}}\right) x F_{3}\right)\right]
$$

where the propagator term was dropped out. To ease the notations, we define the term that includes the longitudinal structure function $R_{L}$ as

$$
\tilde{R} \equiv \frac{1+\frac{2 M x}{\nu}}{1+R_{L}}-\frac{M x}{\nu}-1
$$

Integration over $x$ for fixed $\nu$ and $E_{\nu}$ gives:

$$
\begin{aligned}
\frac{d \sigma^{\nu(\bar{\nu})}}{d \nu} & =\underbrace{\frac{G^{2} M}{\pi} \int F_{2} d x}_{A}-\frac{\nu}{E_{\nu}} \underbrace{\frac{G^{2} M}{\pi}\left(\int F_{2} d x \mp \int x F_{3} d x\right)}_{B} \\
& +\frac{\nu^{2}}{2 E_{\nu}^{2}} \underbrace{\frac{G^{2} M}{\pi}\left(\int F_{2} d x \pm \int x F_{3} d x-\int \tilde{R} F_{2} d x\right)}_{C} .
\end{aligned}
$$

In the limit $\nu \rightarrow 0$ the differential number of events is proportional to the relative flux.

$$
\frac{d N}{d \nu}=A \Phi\left(E_{\nu}\right)\left(1+\frac{B}{A} \frac{\nu}{E_{\nu}}-\frac{C}{A} \frac{\nu^{2}}{2 E_{\nu}^{2}}\right) \rightarrow \Phi\left(E_{\nu}\right) A
$$

Substituting the coefficient $C$ in terms of $A$ and $B$ the relation can be rearranged as

$$
\frac{d N}{d \nu}=A \Phi\left(E_{\nu}\right)\left(1+\frac{B}{A}\left(\frac{\nu}{E_{\nu}}-\frac{\nu^{2}}{2 E_{\nu}^{2}}\right)+\frac{\nu^{2}}{2 E_{\nu}^{2}} \frac{\int F_{2} \tilde{R} d x}{\int F_{2} d x}\right)
$$

where

$$
\frac{B}{A}=-\left(1+\frac{\int x F_{3} d x}{\int F_{2} d x}\right)
$$


Integrating Eq. (3.37) over $\nu$ yields the following relation for the relative flux:

$$
\left.N\left(E_{\nu}\right)\right|_{\nu<\nu_{0}}=\Phi\left(E_{\nu}\right) \int_{0}^{\nu_{0}} d \nu A\left[1+\frac{B}{A}\left(\frac{\nu}{E}-\frac{\nu^{2}}{2 E_{\nu}^{2}}\right)+\frac{\nu^{2}}{2 E_{\nu}^{2}} \frac{\int F_{2} \tilde{R} d x}{\int F_{2} d x}\right],
$$

where the integration is performed up to a fixed $\nu_{0}$ cut chosen to ensure enough statistics in high energy bins $\left(\nu_{0}=20 \mathrm{GeV}\right)$. Hence, the energy dependence of the incident neutrino flux is directly proportional to integrated number of events in energy bins up to some small corrections in powers of $\frac{\nu}{E}$.

\subsubsection{Flux Extraction}

For the extraction of the flux, the raw number of events from the flux sample (see Sect. 3.1.3.1) has to be corrected for detector acceptance and resolution effects using a Monte Carlo simulation. The events are binned in $E_{\nu}, E_{h a d}$ bins to form a $\frac{d N(E)}{d \nu}$ distribution for a given $E_{\nu}$. The relative flux is then determined from:

$$
\Phi\left(E_{\nu}\right) \propto \int_{0}^{\nu_{0}} \frac{\frac{d N(E)}{d \nu}}{1+\left\langle\frac{B}{A}\right\rangle\left(\frac{\nu}{E}-\frac{\nu^{2}}{2 E_{\nu}^{2}}\right)+\frac{\nu^{2}}{2 E_{\nu}^{2}} \frac{\int F_{2} \tilde{R} d x}{\int F_{2} d x}} d \nu
$$

where $\frac{\nu}{E_{\nu}}$ is the correction accounting for energy dependence. The integral term $\frac{\int F_{2} \tilde{R}_{d x}}{\int F_{2} d x}$ from the denominator is calculated using PDFs from our cross section model. The $\frac{B}{A}$ term is obtained by a fit to $\frac{d N}{d \nu}$ data for each energy bin corrected for the radiative effects, charm mass, isoscalar target, and propagator (as described in 3.2.1). These corrections need to be applied because the expression which relates $\frac{B}{A}$ to $\frac{d N}{d \nu}$ assumes a bare DIS differential cross-section with massless quarks.

The fit is performed over $5<\nu<20 \mathrm{GeV}$ range for each neutrino energy bin. The lower bound is chosen to reduce the quasi-elastic and resonance contributions. The average 
$\left\langle\frac{B}{A}\right\rangle$ is determined by averaging $\frac{B}{A}$ from fits in all energy bins separately for neutrinos and antineutrinos, obtaining the following values [22]:

$$
\begin{aligned}
& <B / A>^{\nu}=-0.34 \pm 0.04 ; \\
& <B / A>^{\bar{\nu}}=-1.68 \pm 0.03 .
\end{aligned}
$$

\subsubsection{Flux normalization}

Once the relative flux is determined, the total neutrino cross section can be extracted by:

$$
\frac{\sigma}{E_{\nu}}=\frac{N^{D A T A}}{E_{\nu} \Phi\left(E_{\nu}\right)}
$$

where $N^{D A T A}$ is the number of events from a data sample that selects all the events containing a muon, corrected for detector acceptance and resolution effects (see Ref. [22] for more details).

The absolute incident neutrino flux is determined by normalizing the measured total neutrino cross section to the world average neutrino cross-section for an isoscalar target [50]:

$$
\frac{\sigma_{w o r l d}^{\nu}}{E}=0.677 \pm 0.014 \times 10^{-38} \mathrm{~cm}^{2} / \mathrm{GeV}
$$




\subsection{DIFFERENTIAL CROSS-SECTION EXTRACTION}

The differential cross section for inclusive charge current neutrino iron scattering is extracted from the cross section data sample. The events are binned in $12 x$ bins, $11 y$ bins, and $7 E_{\nu}$ bins. The average differential cross section for each kinematic bin can be expressed in terms of number of events and neutrino flux as

$$
\left\langle\frac{d^{2} \sigma}{E_{\nu} d x d y}\right\rangle_{i j k}=\frac{X S E C_{i j k}^{D A T A} \times C O R R_{-} A C C_{i j k}}{\Phi\left(E_{\nu}^{k}\right) E_{\nu}^{k} \Delta x_{i} \Delta y_{j}}
$$

where $i, j, k$ represents the $x, y, E_{\nu}$ bins and $\Delta x, \Delta y$ are the widths of $x$ and $y$ bins chosen according to the event population and detector resolution. $\operatorname{XSEC}^{D A T A}\left(x, y, E_{\nu}\right)$ is the number of raw data events in each kinematic bin of teh cross section sample defined in Sect. 3.1.3.2. The NUMONTE program is used to correct the binned cross section data sample for detector acceptance and resolution effects. This correction is defined as follows:

$$
C O R R \_A C C_{i j k}=\frac{G E N_{i j k}^{D X S E C}}{S M E_{i j k}^{D X S E C}},
$$

where $G E N_{i j k}^{D X S E C}$ and $S M E_{i j k}^{D X S E C}$ are the generated and the smeared number of events in each bin of the high statistics Monte Carlo sample (more than 20 times data statistics). Also, a bin centering correction has to be applied to correct for the number of events at the average value of each kinematic bin versus the value at the bin center:

$$
C O R R \_B C_{i j k}=\frac{\frac{d^{2} \sigma}{E d x d y}\left(x_{i}^{C}, y_{i}^{C}, E_{k}^{C}\right)}{\frac{d^{2} \sigma}{E d x d y}\left(x_{i}^{a v e}, y_{i}^{a v e}, E_{k}^{a v e}\right)},
$$

where $x_{i}^{C}=\left(x_{i+1}+x_{i}\right) / 2, y_{j}^{C}=\left(y_{j+1}+y_{j}\right) / 2, E_{k}^{C}=\left(E_{k+1}+E_{k}\right) / 2$. 


\subsubsection{Iteration Procedure}

Since Monte Carlo needs to be used both in neutrino flux extraction and in differential cross section extraction to account for detector effects, the process has to be iterated. The iteration procedure is as follows:

- Input an initial flux and PDF parameters for the cross section model from CCFR, the predecessor of the $\mathrm{NuTeV}$ experiment [51].

- Generate Monte Carlo events using the NUMONTE program. Produce acceptance corrections.

- Correct the flux and cross section samples for acceptance and resolution effects.

- Perform a fit to the differential cross-section and extract a new set of PDF parameters.

- Calculate the radiative corrections using the new PDFs and extract a new flux vertex distribution.

- Repeat this procedure till relative change in the differential cross section averaged over all data points is less that $0.1 \%$. Require at least $20 \%$ acceptance for each kinematic bin on last iteration.

For the final iteration the final goodness of the fit per degree of freedom was found to be $\chi^{2} /$ dof $=\mathbf{2 2 2 5} / \mathbf{2 5 9 9}$, which includes the systematic uncertainties. There are 1423 number of neutrino differential cross section points and 1195 number of antineutrinos. The final fit results of the cross-section model parameters are shown in Table 3.2. Figures 3.6, 3.7 show an example of the extracted differential cross section distribution for neutrino and antineutrino scattering on iron as function of inelasticity $y$ in various $x$ bins, at $110 \mathrm{GeV}$ and $190 \mathrm{GeV}$ neutrino energy [22]. 


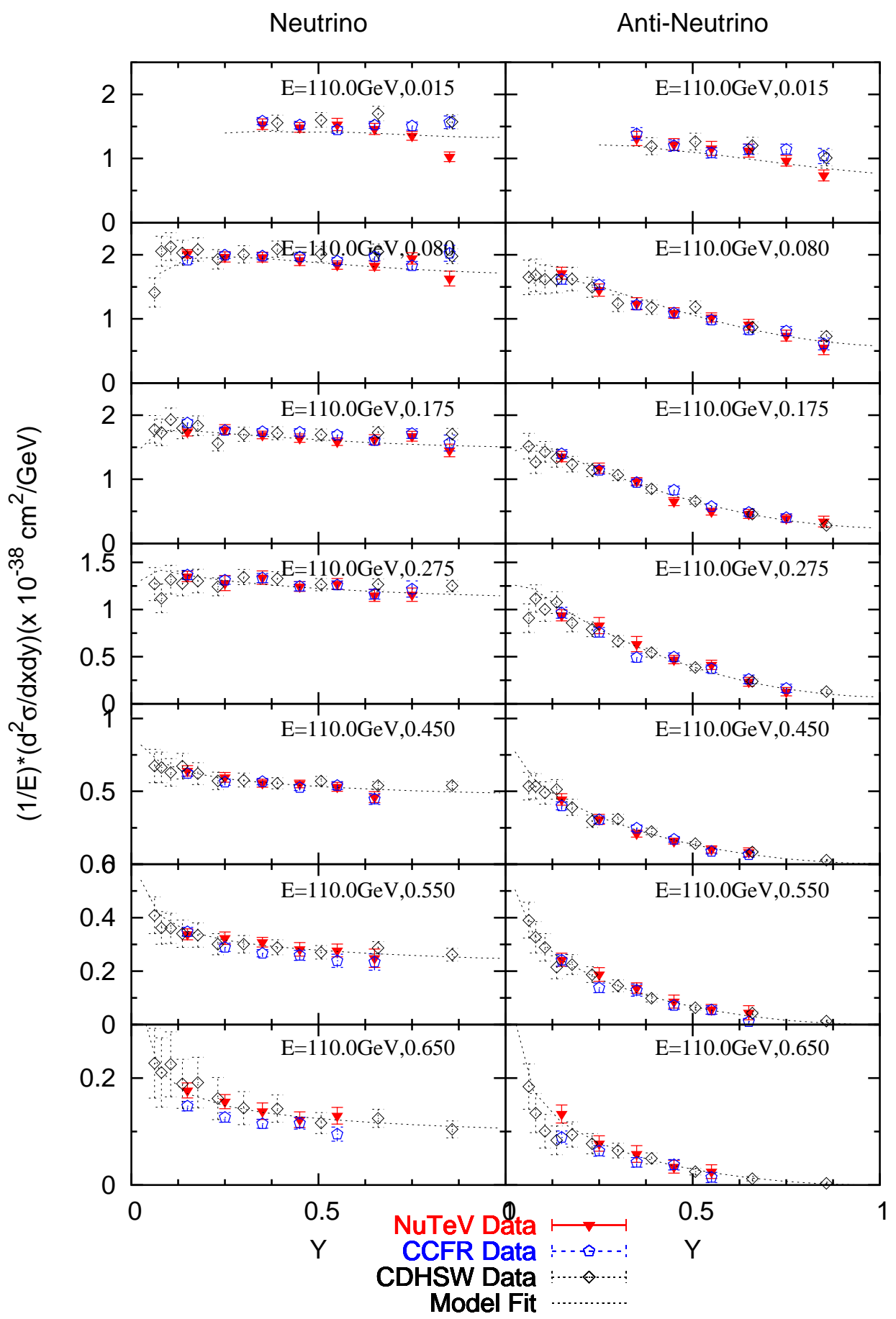

Figure 3.6: Comparison of $\mathrm{NuTeV}$ neutrino and antineutrino differential cross section (red triangles) to previous neutrino measurements on iron (CCFR -blue circles, CDHSW -black diamonds) as function of $y$ for various $x$ bins. This is shown for $190 \mathrm{GeV}$ neutrino energy. 


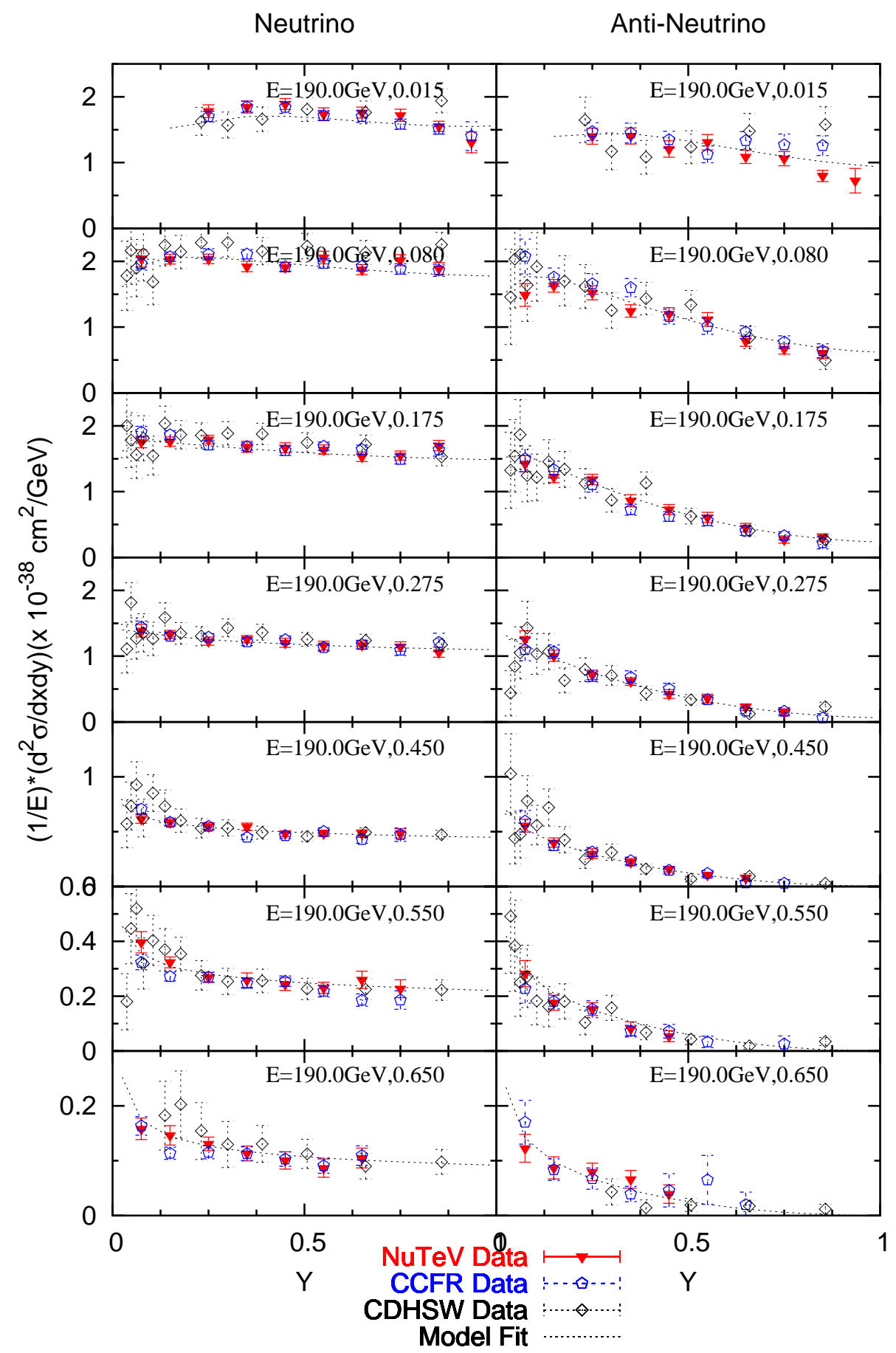

Figure 3.7: Comparison of $\mathrm{NuTeV}$ neutrino and antineutrino differential cross section (red triangles) to previous neutrino measurements on iron (CCFR -blue circles, CDHSW -black diamonds) as function of $y$ for various $x$ bins. This is shown for $190 \mathrm{GeV}$ neutrino energy. 


\subsection{FLUX AND CROSS SECTION SYSTEMATIC UNCERTAINTIES}

The following sources of systematic uncertainties are considered in the extraction procedure of the differential cross-section extraction:

- Muon energy scale (0.7\%): affects both flux and cross-section extraction. To take the effect of this systematic into account, the data for both cross section and flux samples are shifted by $\pm 1 \sigma$ and then re-extract the flux and cross-section. The result is compared to the nominal (default) cross-section.

- Hadron energy scale $(0.43 \%)$ : affects both flux and cross-section extraction. The effect of this systematic is taken into account by shifting both cross section and flux samples by $\pm 1 \sigma$ and re-extract both the differential cross section and flux.

- Charm mass: is used in the charm production model to correct the flux sample to extract flux. The uncertainty on $m_{c}$ is taken to be $\delta m_{c}=0.18 \mathrm{GeV}$. The effect of the systematic is evaluated by shifting the value by $\pm 1 \sigma$ up and down and extract differential cross section for each shift.

- $\boldsymbol{B} / \boldsymbol{A}$ : is the statistical error on the $\frac{d N}{d \nu}$ fit to the flux sample. It only affects the flux measurement. The effect of this systematic is evaluated by shifting $\frac{B}{A}$ by $1 \sigma$ and re-extract flux. Using the shifted flux, a cross section is extracted and the output is compared to the nominal cross-section.

- Muon energy smearing: affects the acceptance correction from used to correct the raw data. Its effect is evaluated by taking the difference between two models: NuTeV smearing function and CCFR smearing function for muon energy smearing.

- Hadron energy smearing: affects the acceptance correction used to correct the raw data. The hadron smearing function is determined from a fit to the calibration data. The error from the fit is used to change the hadron energy response by $\pm 1 \sigma$.

- Flux normalization: the uncertainty in the absolute flux determination is taken to be 
$2.1 \%$ (from the normalization to the world average neutrino cross-section). This is set to be the overall normalization of the experiment.

The dominant systematic errors come from the uncertainties in the muon and hadron energy scales. The uncertainties in the parameters of the cross section model are consistent with statistical fluctuations and have been neglected.

A point-to-point covariance matrix was calculated to account for the correlations between data points, which are large for neighboring bins. The covariance matrix is constructed as follows:

$$
M_{\alpha \beta}=\sum_{i=1}^{7} \delta_{i \mid \alpha} \delta_{i \mid \beta},
$$

where $\delta_{i \mid \beta}$ corresponds to $1 \sigma$ shift in data point $\alpha$ due to systematic uncertainty $i$. The statistical error is added in quadrature to the diagonal elements of the covariance matrix. $\mathrm{NuTeV}$ designed a package with a user friendly interface called $\mathrm{NuTeV}$ pack. It provides access to its measured neutrino and anti-neutrino differential cross section on Iron together with a full covariance error matrix which accounts for all systematic errors. NuTeVpack has the flexibility of turning $\mathrm{ON}$ and $\mathrm{OFF}$ any of the considered systematic uncertainties.

\subsection{STRUCTURE FUNCTIONS EXTRACTION}

Structure functions can be obtained from 1-parameter or 2-parameter fits to the $y$ dependence of the linear combination of measured neutrino and antineutrino differential cross sections. The relation that connects structure functions to differential cross sections is based on the DIS bare differential cross section relation, i.e. Eq. (1.27). Therefore, before performing the fits data needs to to be corrected for non-isoscalarity, radiative, and massive propagator 
effects as described in Sect. 3.2.1.2. The corrections are applied as multiplicative factors to the extracted differential cross section data

$$
C_{\text {bare }}(x, y, E)=\frac{\frac{d^{2} \sigma}{d x d y}\left(\mathrm{ISO}, M_{W}=0 \mathrm{GeV}, \text { no rad. corr. }\right)}{\frac{d^{2} \sigma}{d x d y}\left(\mathrm{Fe}, M_{W}=80.4 \mathrm{GeV}, \text { rad. corr. }\right)}
$$

Data is then re-binned from $(E, x, y)$ to express the structure functions as function of $x$ and $Q^{2}$. The bin-center correction is given by

$$
C_{Q^{2}}=\frac{\frac{d^{2} \sigma}{d x d y}\left(x, y, Q_{c}^{2}\right)}{\frac{d^{2} \sigma}{d x d y}\left(x, y, Q^{2}=2 M x y E\right)} .
$$

The procedures of extracting the nucleon structure functions used in the QCD analysis are presented below.

\subsubsection{From 1-Parameter Fit}

Nucleon structure function $x F_{3}$ is obtained from one-parameter fit to $y$-dependence of the following combination of the cross sections:

$$
\begin{aligned}
& {\left[\frac{d^{2} \sigma^{\nu}}{d x d y}-\frac{d^{2} \sigma^{\bar{\nu}}}{d x d y}\right] \frac{\pi}{2 M G^{2} E_{\nu}}=\Delta F_{2}\left(1-y-\frac{M x y}{2 E}+\frac{1+\frac{4 M^{2} x^{2}}{Q^{2}}}{1+R_{L}} \frac{y^{2}}{2}\right)+\left(y-\frac{y^{2}}{2}\right) x F_{3}^{a v g},(3} \\
& F_{2(3)}^{a v g}\left(x, Q^{2}\right)=\frac{1}{2}\left(F_{2(3)}^{\nu}\left(x, Q^{2}\right)+F_{2(3)}^{\bar{\nu}}\left(x, Q^{2}\right)\right), \\
& \Delta F_{2}=F_{2}^{\nu}-F_{2}^{\bar{\nu}} \approx 0
\end{aligned}
$$

$\Delta F_{2}$ is very small and is neglected, thus no need to input model for extracting $x F_{3}$. The resulting data table for the $x F_{3}$ structure function is presented in the Appendix B.1. Structure function $x F_{3}$ from one-parameter fit is used in the Non-Singlet QCD fit to determine $\Lambda_{Q C D}$ (see Sect. 4.2.2). 


\subsubsection{From 2-Parameter Fit}

This is a new method of extracting the structure functions, and thus of $\Lambda_{Q C D}$. One also could extract structure functions from 2-parameter fits to the $\nu(\bar{\nu})$ cross sections as follows:

$$
\begin{aligned}
\frac{d^{2} \sigma^{\nu}}{d x d y} & =\frac{2 M G^{2} E_{\nu}}{\pi}\left[\left(1-y-\frac{M x y}{2 E}+\frac{1+\frac{4 M^{2} x^{2}}{Q^{2}}}{1+R_{L}} \frac{y^{2}}{2}\right)\left(F_{2}^{a v g}+\frac{\Delta F_{2}}{2}\right)\right. \\
& \left.+y\left(1-\frac{y}{2}\right)\left(x F_{3}^{a v g}+\frac{\Delta x F_{3}}{2}\right)\right], \\
\frac{d^{2} \sigma^{\bar{\nu}}}{d x d y} & =\frac{2 M G^{2} E_{\nu}}{\pi}\left[\left(1-y-\frac{M x y}{2 E}+\frac{1+\frac{4 M^{2} x^{2}}{Q^{2}}}{1+R_{L}} \frac{y^{2}}{2}\right)\left(F_{2}^{a v g}-\frac{\Delta F_{2}}{2}\right)\right. \\
& \left.+y\left(1-\frac{y}{2}\right)\left(x F_{3}^{a v g}-\frac{\Delta x F_{3}}{2}\right)\right],
\end{aligned}
$$

$$
\begin{aligned}
F_{2}^{a v g}\left(x, Q^{2}\right) & =\frac{1}{2}\left(F_{2}^{\nu}\left(x, Q^{2}\right)+F_{2}^{\bar{\nu}}\left(x, Q^{2}\right)\right) \\
x F_{3}^{a v g}\left(x, Q^{2}\right) & =\frac{1}{2}\left(x F_{3}^{\nu}\left(x, Q^{2}\right)+x F_{3}^{\bar{\nu}}\left(x, Q^{2}\right)\right) \\
\Delta x F_{3} & =x F_{3}^{\nu}-x F_{3}^{\bar{\nu}} \\
\Delta F_{2} & =F_{2}^{\nu}-F_{2}^{\bar{\nu}}
\end{aligned}
$$

For this case, input models for $\Delta x F_{3}$ and $R_{L}$ are needed. For $\Delta x F_{3}$ we use a NLO model from TRVFS with MRST99 PDF set (see Ref.[69]). Note that for $x>0.1, \Delta x F_{3}$ is negligible. For $R_{L}$ a parametrization of world data is used (see Eq. (3.20)) [46]. The simultaneous extraction of $F_{2}$ and $x F_{3}$ uses the full covariance error matrix of the differential cross sections described in Sect. 3.5. A separate $\chi^{2}$ is constructed for neutrino and antineutrino fits by minimizing the above relations. The resulting data tables for the $F_{2}$ abd $x F_{3}$ structure functions are presented in the Appendix B.2. These structure functions are used in the Combined QCD fits to determine $\Lambda_{Q C D}$ (see Section 4.2.3). 


\subsubsection{Structure Functions Systematic Uncertainties}

The sources for the systematic uncertainties in extracting the structure functions originate from the extraction of the differential cross sections presented in Section 3.5. For the structure functions from two-parameter fits there are additional systematic errors due to the input models for $\Delta x F_{3}$ and $R_{L}$. The systematic uncertainty due to $\Delta x F_{3}$ model is evaluated by taking the difference between structure functions extracted with two different NLO models of $\Delta x F_{3}$ : TRVFS [69] and ACOTFFS [12]. The systematic uncertainty due to $R_{L}$ parametrization is evaluated by shifting its value by $\pm 15 \%$ (which corresponds to 1 sigma uncertainty in the parametrization) and re-extracting the structure functions for each shift. 


\section{0 $\Lambda_{Q C D}$ FROM NLO QCD FITS}

Once the structure functions have been obtained, we can proceed to QCD analysis. In this Chapter we present the methods of extracting the fundamental parameter of QCD, $\Lambda_{Q C D}$ and the fit results. The $\Lambda_{Q C D}$ parameter is determined from two types of NLO QCD fits. The "non-singlet" fit uses only $x F_{3}\left(x, Q^{2}\right)$, while the "combined" fit uses both $F_{2}\left(x, Q^{2}\right)$ and $x F_{3}\left(x, Q^{2}\right)$ structure functions and their correlation. The non-singlet fit is independent of any assumption about gluon distribution. The combined fit has the advantage of improved statistical precision for $\Lambda_{Q C D}$.

The fits are performed using two theoretical models: the massless $\overline{M S}$ scheme (referred to as $\overline{M S}$ model in the rest of this work) [52], and, for the first time, a model that takes into account heavy quark production using a scheme devised by Aivazis-Collins-Olness-Tung (referred to as ACOT model) [12]. Both models have been described in section 1.3.1.

\subsection{QCD PROGRAM}

The NLO QCD fits are performed using a fitting FORTRAN program $[53,54]$ that allows the user to choose between $A C O T$ and $\overline{M S}$ models. Also, this program includes a complete treatment of the correlations between $F_{2}\left(x, Q^{2}\right)$ and $x F_{3}\left(x, Q^{2}\right)$. The program can be dissected in the following building blocks: the PDF parametrization and evolution, the NLO calculations of the structure functions using either $\overline{M S}$ or $A C O T$ model, and the minimiza- 
tion of $\chi^{2}$ for either uncorrelated or correlated case. A schematic flow of the program is illustrated in Figure 4.1. Each of these components is discussed in detail in the following Sections.

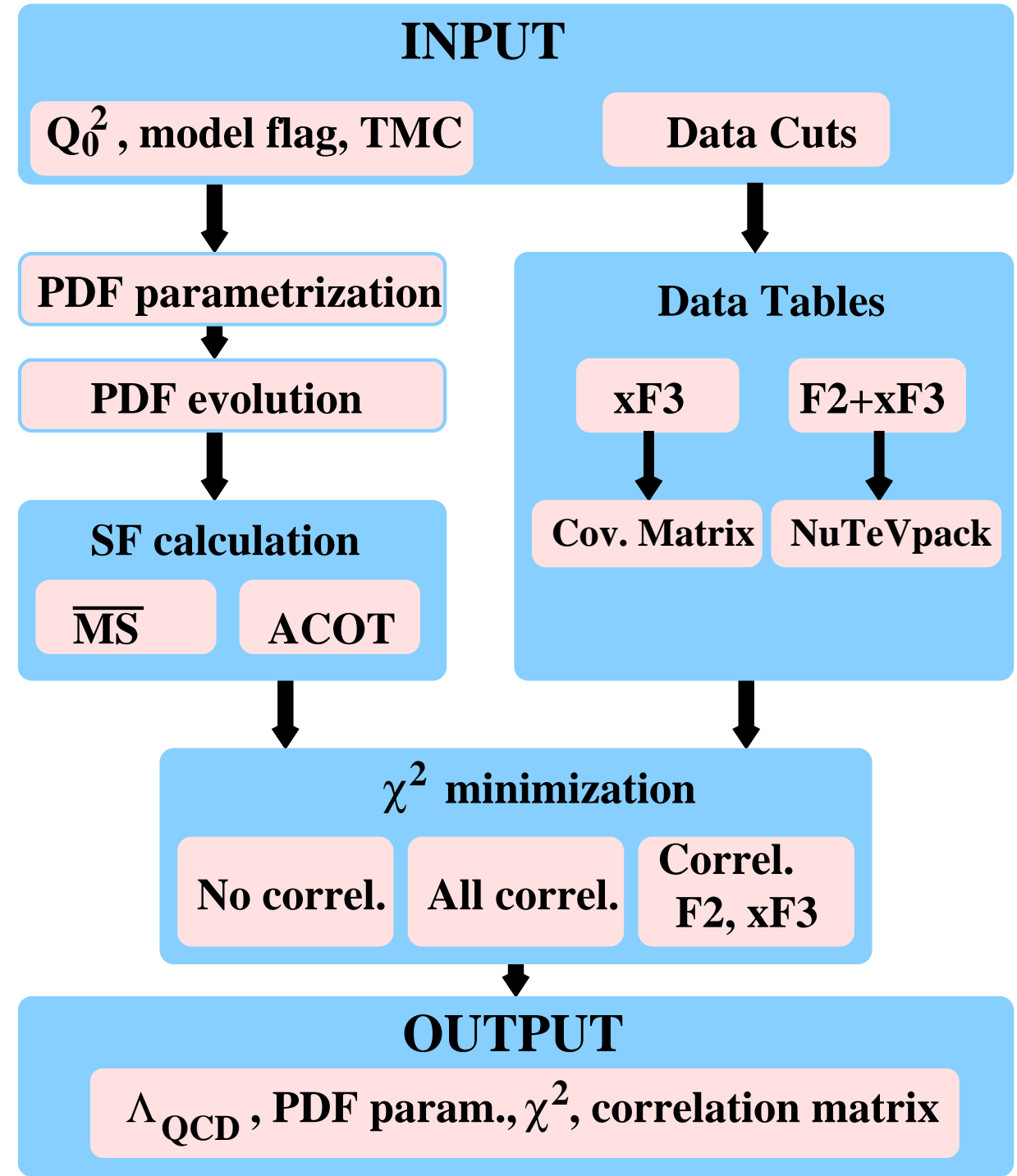

Figure 4.1: A Flow-chart of the NLO QCD fitting code. 


\subsubsection{PDF Parametrization and Evolution}

An initial parametrization of the PDFs at a reference scale $Q^{2}$ is a required input to the QCD program. This reference scale is chosen here to be $Q_{0}^{2}=5 \mathrm{GeV}^{2}$ to minimize the non-perturbative QCD effects. For the non-singlet fit we use the following functional form

$$
x q^{N S}=x u_{v}+x d_{v}=\left(A 0_{u_{v}}+A 0_{d_{v}}\right) x^{A 1_{u_{v}}}(1-x)^{A 2_{u_{v}}},
$$

where $N S$ refers to non-singlet distribution. For the combined fit, we also need to input a parametrization for singlet and gluon distributions such as

$$
\begin{aligned}
x q^{S} & =\underbrace{x u_{v}+x d_{v}}_{x q^{N S}}+2 A 0_{u d}(1-x)^{A 2_{u d}}, \\
x G & =A 0_{g}(1-x)^{A 2_{g}}
\end{aligned}
$$

where $S$ refers to singlet distribution and $G$ to gluon distribution. A more general parametrization was first tried, then the parameters for which the fit was not sensitive to were removed from the fits after initial studies (this is discussed further in Section 4.3.1).

The PDFs must satisfy the QCD sum rules, which were described in Section 1.3.3. However, NuTeV data covers a limited $x$ range. Therefore, it does not directly constrain the PDFs via sum rules. Consequently, one must choose either to impose the QCD sum rules on the fitting function or to check if the fitted PDFs satisfy the sum rules. This analysis imposes the QCD sum rules on the fitting functions. As a result, the normalization parameters $A 0_{u_{v}}, A 0_{d_{v}}$, and $A 0_{g}$ are constrained by these rules. For example, Eq. (1.77) constrains $A 0_{u_{v}}, A 0_{d_{v}}$, and Eq. (1.78) constrains $A 0_{g}$. Thus, the only free parameters for the non-singlet fit are $A 1_{u_{v}}, A 2_{d_{v}}, \Lambda_{Q C D}$, while for the combined fit these are $A 1_{u_{v}}, A 2_{d_{v}}, A 0_{u d}$, $A 2_{u d}, A 2_{g}, \Lambda_{Q C D}$. The $\Lambda_{Q C D}$ parameter enters into the fit as a free parameter via evolution Equations (1.74)-(1.76). In addition, there is an overall normalization parameter, "norm", which is allowed to float freely. 
Once the functional forms for PDFs are defined, the initial PDFs are evaluated at a reference point $Q_{0}^{2}=5 \mathrm{GeV}^{2}$ with the initial values for the fit parameters ranging between 0.8 and 5 , with $\Lambda_{Q C D}=337 \mathrm{MeV}$. The fit does not depend on the choice of starting values. Then the parametrized PDFs are evolved following the steps below:

- Apply evolution Equations (1.74), (1.75), (1.76) to the initial PDFs to obtain $\partial q(x, s) / \partial s$ at $Q^{2}=Q_{0}^{2}$ (i.e. $s=0$, where $s=\ln \left[\ln \left(Q^{2} / \Lambda^{2}\right) /\left[\ln \left(Q_{0}^{2} / \Lambda^{2}\right)\right]\right.$ ).

- Use a predictor-corrector algorithm to estimate the PDFs for incrementally increased $s$, $q(x, s+\delta s)[55]$

- Repeat until the whole kinematic range is covered.

The evolved PDFs are then stored in a grid in $\left(x_{i}, s_{i}\right)$ and the values of the PDFs at any arbitrary $(x, s)$ are obtained via a linear interpolation in $x, s$ from the grid. These PDFs are used to calculate the NLO structure functions.

\subsubsection{QCD Models}

The NLO QCD models used in this analysis $(\overline{M S}$ and $A C O T)$ were presented in Section 4.1.2. The NLO structure functions are obtained from convolution of the parametrized PDFs with the hard scattering coefficients. Since neutrino scattering is sensitive to heavy quark production, the primary result of this measurement uses the $A C O T$ model, while the $\overline{M S}$ model is only used to observe the differences between the massless and finite quark mass treatments. Throughout this analysis the following values for quark masses are used: for bottom quark $m_{b}=4.3 \pm 0.2 \mathrm{GeV}$ (taken from 2005 Review Particle Listing [4]) and for charm quark $m_{c}=1.4 \pm 0.2 \mathrm{GeV}$ (obtained from a weighted average of LO experimental measurements [39], [48]). The latter one is chosen to be consistent with the extraction method of the differential cross section (see Sect. 3.2.1.2). Also, the $\mathrm{CKM}^{1}$ matrix elements

\footnotetext{
${ }^{1}$ CKM stands for Cabibbo-Kobayashi-Maskawa. CKM is the matrix that relates the quark mass eigenstates to the weak eigenstates
} 
used in the program are the average values from Ref. [4],

$$
V=\left(\begin{array}{ccc}
V_{u d} & V_{u s} & V_{u b} \\
V_{c d} & V_{c s} & V_{c b} \\
V_{t d} & V_{t s} & V_{t b}
\end{array}\right)=\left(\begin{array}{ccc}
0.97485 & 0.2225 & 0.00365 \\
0.22250 & 0.9740 & 0.04100 \\
0.00900 & 0.0405 & 0.99915
\end{array}\right) .
$$

\subsubsection{Target Mass Correction}

The QCD models do not account for target mass effects. Hence, the models should be corrected to fit data which includes these effects. Target mass effects are only important at low $Q^{2}$ and high $x$, as described in Sect. 1.3.4.1. For the ACOT model, the corrections are implemented in the QCD program for the structure functions $F_{2}\left(x, Q^{2}\right)$ and $F_{3}\left(x, Q^{2}\right)$ using Eqs. (1.80) and (1.81). Since the $\overline{M S}$ model returns in the program $x F_{3}\left(x, Q^{2}\right)$ instead of $F_{3}\left(x, Q^{2}\right)$, Eq. (1.81) is adjusted as follows:

$$
\begin{aligned}
x F_{3}^{T M} & =x \frac{x}{\xi} \frac{F_{3}(\xi)}{k^{2}}+x \frac{2 M_{p}^{2} x^{2}}{Q^{2} k^{3}} \int_{\xi}^{1} \frac{F_{3}(u)}{u} d u \\
x F_{3}^{T M} & =\left(\frac{x}{\xi}\right)^{2} \frac{\xi F_{3}(\xi)}{k^{2}}+\frac{2 M_{p}^{2} x^{3}}{Q^{2} k^{3}} \int_{\xi}^{1} \frac{u F_{3}(u)}{u^{2}} d u .
\end{aligned}
$$

The effect of including the target mass correction on the measurement of $\Lambda_{Q C D}$, in the $\mathrm{NuTeV}$ kinematic range, was studied (see Section 4.3.4) and was found to be negligible.

\subsection{3 $\chi^{2}$ Minimization}

In this analysis the minimization of the $\chi^{2}$ is based on the Marquardt fitting algorithm [56]. It behaves like a gradient search far from the minimum and like a linear least squares near the minimum. The fitting program allows a treatment for the correlations between $F_{2}$ and $x F_{3}$, as well as for the point-to-point correlations. We discuss here three possibilities. 


\subsubsection{No Correlations Case}

This method is used for the non-singlet fit when only statistical errors are taken into account, and also when the weight of each contributing systematic error is evaluated separately. The $\chi^{2}$ for this case is

$$
\chi^{2}=\sum_{i} \frac{1}{\sigma_{i}}\left(F_{i}^{D A T A}-F^{T H}\left(x_{i}\right)\right)^{2},
$$

where $F_{i}^{D A T A}$ corresponds to $x F_{3}$ data points, $\sigma_{i}$ to $x F_{3}$ statistical errors, and $F^{T H}\left(x_{i}\right)$ to theoretical $x F_{3}$ which depends on the fit parameters associated with PDF parametrization. The index $i$ is over the total number of data points (i.e., $i=1, \ldots, 63$ ). The minimization process uses the linearized function obtained by Taylor expansion of $F^{T H}\left(x_{i}\right)$ to first order as,

$$
F^{T H}\left(x_{i}\right)=F^{\prime}\left(x_{i}\right)+\sum_{j=1}^{m}\left(\frac{\partial F^{\prime}\left(x_{i}\right)}{a_{j}} \delta a_{j}\right),
$$

where index $j$ runs over the number of fit parameters. Hence, the $\chi^{2}$ can be re-written as

$$
\chi^{2}=\sum_{i}\left(\frac{1}{\sigma_{i}^{2}}\left[F_{i}^{D A T A}-F^{\prime}\left(x_{i}\right)-\sum_{j=1}^{m}\left(\frac{\partial F^{\prime}\left(x_{i}\right)}{a_{j}} \delta a_{j}\right)\right]^{2}\right) .
$$

The derivative of the $\chi^{2}$ is computed for each parameter increment, $\delta a_{j}$. A set of equations is then obtained:

$$
\frac{\partial \chi^{2}}{\partial \delta a_{k}}=-2 \sum_{i}\left(\frac{1}{\sigma_{i}^{2}}\left[F_{i}^{D A T A}-F^{\prime}\left(x_{i}\right)-\sum_{j=1}^{m}\left(\frac{\partial F^{\prime}\left(x_{i}\right)}{a_{j}} \delta a_{j}\right)\right] \frac{\partial F^{\prime}\left(x_{i}\right)}{\partial a_{k}}\right)=0 .
$$

The results can be expressed in matrix elements form as

$$
\beta_{k}=\delta a_{j} \alpha_{j k}
$$

where

$$
\begin{aligned}
\beta_{k} & =\sum_{i}\left(\frac{1}{\sigma_{i}^{2}}\left[F_{i}^{D A T A}-F^{\prime}\left(x_{i}\right)\right] \frac{\partial F^{\prime}\left(x_{i}\right)}{\partial a_{k}}\right), \\
\alpha_{j k} & =\sum_{i}\left(\frac{1}{\sigma_{i}^{2}} \frac{\partial F^{\prime}\left(x_{i}\right)}{\partial a_{j}} \frac{\partial F^{\prime}\left(x_{i}\right)}{\partial a_{k}}\right) .
\end{aligned}
$$


$\alpha_{j k}$ represents the symmetric curvature matrix.

In Marquardt algorithm the interpolation between gradient search and analytical methods is done by adding to the diagonal terms of the curvature matrix $\left(\alpha_{j k}\right)$ a factor $1+\lambda$, as follows:

$$
\alpha_{j k}^{\prime}= \begin{cases}\alpha_{j k}(1+\lambda) & \text { for } j=k \\ \alpha_{j k} & \text { for } j \neq k\end{cases}
$$

The solution for each $j$ parameter increment $\delta a_{j}$ is obtained as

$$
\delta a_{j}=\sum_{k=1}^{m}\left(\beta_{k} \epsilon_{j k}^{\prime}\right)
$$

where the error matrix $\boldsymbol{\epsilon}=\boldsymbol{\alpha}^{-1}$ is the inverse of the curvature matrix. If $\lambda$ is very small the solution is similar to the analytical case described above. If $\lambda$ is very large, then the diagonal terms dominate and the matrix equation degenerates into $m$ separate equations:

$$
\beta_{j}=\lambda \delta a_{j} \alpha_{j j}
$$

The uncertainty in parameter $a_{j}$ corresponds to $\sigma_{a_{j}}=\epsilon_{j j}$.

The minimization recipe given by Marquardt $[56,55]$ is applied in the program as

1. Calculate $\chi^{2}(a)$ with $\lambda=0.001$.

2. Compute $\delta a$ and $\chi^{2}(a+\delta a)$.

3. If

$$
\left\{\begin{array}{l}
\chi^{2}(a+\delta a)>\chi^{2}(a) \text { then } \lambda \rightarrow 10 \times \lambda \\
\chi^{2}(a+\delta a)<\chi^{2}(a) \text { then } \lambda \rightarrow \frac{\lambda}{10} \text { and } a \rightarrow a^{\prime}=a+\delta \text { go to step } 1 \text { with } \lambda=\lambda / 10
\end{array}\right.
$$




\subsubsection{Point-to-Point Correlations Case}

The minimization method for this case is similar to the uncorrelated case. However, here the point-to-point correlations are taken into account by constructing the structure function covariance error matrix (discussed in Section 4.2.1). The method is only applied for the nonsinglet fit because for the combined fit, the correlations between $F_{2}$ and $x F_{3}$ must also be considered (see Section 4.1.3.3). The general form of the $\chi^{2}$ that includes the full covariance matrix is

$$
\chi^{2}=\sum_{i} \sum_{j}\left(F_{i}^{D A T A}-F^{T H}\left(x_{i}\right)\right) V_{i j}^{-1}\left(F_{j}{ }^{D A T A}-F^{T H}\left(x_{j}\right)\right),
$$

where both indices $i, j$ run over the total number of data points (i.e. $i, j=1, \ldots, 63$ ) and $F_{i}^{D A T A}$ and $F_{i}^{T H}$ have the same meaning as for the previous case. In the following derivations it has been assumed that the covariance matrix is symmetric (i.e. $V_{i j}^{-1}=V_{j i}^{-1}$ ). The fitting function is linearized using a first order Taylor expansion, so that

$$
\begin{aligned}
\chi^{2}= & \sum_{i, j}\left(F_{i}^{D A T A}-F^{\prime}\left(x_{i}\right)-\sum_{m} \frac{\partial F^{\prime}\left(x_{i}\right)}{\partial a_{m}} \delta a_{m}\right) V_{i j}^{-1} \\
& \left(F_{j}^{D A T A}-F^{\prime}\left(x_{j}\right)-\sum_{n} \frac{\partial F^{\prime}\left(x_{j}\right)}{\partial a_{n}} \delta a_{n}\right),
\end{aligned}
$$

with indices $m$ and $n$ corresponding to fit parameters. Then the $\chi^{2}$ is minimized as follows:

$$
\begin{gathered}
\frac{\partial \chi^{2}}{\partial \delta a_{k}}=0 \\
\sum_{i, j}\left(-\frac{\partial F^{\prime}\left(x_{i}\right)}{\partial a_{k}} V_{i j}^{-1}\left(F_{j}-F^{\prime}\left(x_{j}\right)-\sum_{n} \frac{\partial F^{\prime}\left(x_{j}\right)}{\partial a_{n}} \delta a_{k}\right)\right. \\
\left.-\left(F_{i}-F^{\prime}\left(x_{i}\right)-\sum_{m} \frac{\partial F^{\prime}\left(x_{i}\right)}{\partial a_{m}} \delta a_{k}\right) V_{i j}^{-1} \frac{\partial F^{\prime}\left(x_{j}\right)}{\partial a_{k}}\right)=0 .
\end{gathered}
$$

We define $\beta_{k}$ and $\alpha_{k n}$ similarly to the previous case as,

$$
\begin{aligned}
\beta_{k} & =\sum_{i, j}\left(F_{i}-F^{\prime}\left(x_{i}\right)\right) V_{i j}^{-1} \frac{\partial F^{\prime}\left(x_{j}\right)}{\partial a_{k}}, \\
\alpha_{k n} & =\sum_{i, j} \frac{\partial F^{\prime}\left(x_{i}\right)}{\partial a_{k}} V_{i j}^{-1} \frac{\partial F^{\prime}\left(x_{j}\right)}{\partial a_{n}} .
\end{aligned}
$$


Then, Eq. (4.17) can be rewritten as

$$
-2 \beta_{k}+\sum_{n} \alpha_{k n} \delta a_{n}+\sum_{m} \alpha_{m k} \delta a_{m}=0
$$

and if we multiply the right hand side by the inverse of $\boldsymbol{\alpha}, \boldsymbol{\epsilon}=\boldsymbol{\alpha}^{-\mathbf{1}}$ we obtain,

$$
2 \sum_{k} \beta_{k} \epsilon_{k l}=\sum_{n} \alpha_{k n} \alpha_{k l}^{-1} \delta a_{n}+\sum_{m} \alpha_{k m} \alpha_{k l}^{-1} \delta a_{m}
$$

For a symmetric covariance error matrix (i.e. $\left.V_{i j}^{-1}=V_{j i}^{-1}\right) \alpha_{k n}=\alpha_{n k}$ and

$$
\begin{aligned}
2 \sum_{k} \beta_{k} \epsilon_{k l} & =\sum_{n} \delta_{l n} \delta a_{n}+\sum_{m} \delta_{l m} \delta a_{m} \\
2 \sum_{k} \beta_{k} \epsilon_{k l} & =2 \delta a_{l} .
\end{aligned}
$$

Therefore, the solution for the parameter increment $\delta a_{l}$ has the same form as one for the uncorrelated case,

$$
\delta a_{l}=\sum_{k} \beta_{k} \epsilon_{k l}
$$

\subsubsection{Correlation between $F_{2}$ and $x F_{3}$ Case}

In this case, one takes into account the correlations between $F_{2}$ and $x F_{3}$ which were obtained by fitting the same set of differential cross section points. This method only applies for the combined fit. The $\chi^{2}$ for this case is expressed as

$$
\chi^{2}=\sum_{i=1}\left(\sum_{l=1}^{2} \sum_{m=1}^{2}\left(F_{l}^{i(D A T A)}-F_{l}^{i(T H)}\right)\left(V_{l m}^{i}\right)^{-1}\left(F_{m}^{i(D A T A)}-F_{m}^{i(T H)}\right)\right) .
$$

The indices $l, m$ are over the number structure functions $F_{2}$ and $x F_{3}$, i.e. $l, m=1$ corresponds to structure function $F_{2}$, while $l, m=2$ to $x F_{3}$. The $i$ index is over the total number of points $(i=1, \ldots, 132)$. The covariance matrix at each $x, Q^{2}$ point is given by

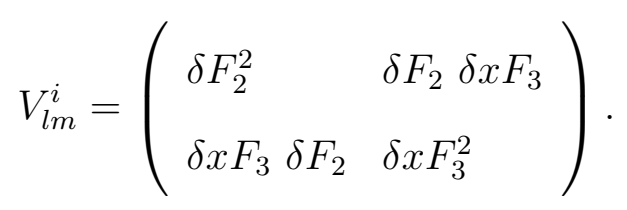


The diagonal elements contain the total error (statistical and systematic) from differential cross section points (described in Sect. 3.6.3 and in Ref. [22]). Additional systematic errors due to model inputs $\left(R_{L}\right.$ and $\left.\Delta x F_{3}\right)$ in extracting the structure functions (described in Section 3.6.2) are added in quadrature to the diagonal elements because they are independent of the cross section uncertainties. The cross terms, $\delta F_{2} \delta x F_{3}$, give the correlated errors between $F_{2}$ and $x F_{3}$.

The minimization of the $\chi^{2}$ is performed similarly to the previous cases, only that, for this case, $\beta$ and $\alpha$ are defined as

$$
\begin{aligned}
\beta_{k} & =\sum_{i=1}\left(\sum_{l, m=1}^{2}\left(F_{l}^{i}-F^{\prime}\left(x_{l}^{i}\right)\right)\left(V_{l m}^{i}\right)^{-1} \frac{\partial F^{\prime}\left(x_{m}^{i}\right)}{\partial a_{k}}\right), \\
\alpha_{k n} & =\sum_{i=1}\left(\sum_{l, m=1}^{2} \frac{\partial F^{\prime}\left(x_{l}^{i}\right)}{\partial a_{k}}\left(V_{l m}^{i}\right)^{-1} \frac{\partial F^{\prime}\left(x_{m}^{i}\right)}{\partial a_{n}}\right) .
\end{aligned}
$$

\section{2 $\Lambda_{Q C D}$ FROM NLO QCD FITS TO STRUCTURE FUNCTIONS}

Here we discuss the results from non-singlet and combined fits. In order to minimize the non-perturbative QCD effects only data points with $Q^{2}>5 \mathrm{GeV}^{2}$ and $W^{2}>10 \mathrm{GeV}^{2}$ are used in the fits. Our data is in the kinematic range of $0.001<x<0.8$ and $5<Q^{2}<125$ $\mathrm{GeV}^{2}$. Fixed-target DIS experiments are not sensitive to bottom quark excitation, therefore a $\Lambda_{Q C D}$ for four active flavors is quoted.

\subsubsection{Structure Function Covariance Matrix}

In order to perform non-singlet fits taking into account the systematic errors, a covariance error matrix for $x F_{3}$ is constructed. The systematic uncertainties for the non-singlet fit originate from the extracted differential cross section data points (see Sec. 3.6.1). Seven sources of systematic errors are considered: the muon and hadron energy scales, the smearing 
models for the muon and hadron energy distributions, and the flux uncertainties which include charm mass and $\frac{B}{A}$ correction uncertainties (see Section 3.5 for details) [22].

To obtain the covariance matrix for $x F_{3}$ each systematic is varied by $\pm 1 \sigma$ (or by using a model difference in some cases, for example, muon energy smearing models). $x F_{3}$ is then reextracted for each shift by going through the whole processes of fitting the differential cross section with each shifted systematic. As a result, one obtains $x F_{3}$ data tables corresponding to each variation of the systematic. Hence, the structure function covariance error matrix is constructed from these shifts as

$$
V_{i j}=\sum_{k=1}^{7} \delta_{k \mid i} \delta_{k \mid j} .
$$

The sum is over all considered systematic shifts (i.e. $k=1, \ldots, 7)$ and $\delta_{k \mid i}$ corresponds to $1 \sigma$ shifts in the data point $i$ due to systematic uncertainty $k$. In other words,

$$
\delta_{k \mid i}=\frac{F_{k}^{+}(i)-F_{k}^{-}(i)}{2}
$$

where $F^{+}\left(F^{-}\right)$denotes the extracted structure functions from the fits to the cross sections shifted by $+1 \sigma(-1 \sigma)$. The statistical errors are added in quadrature to the diagonal elements of the covariance matrix. The $x F_{3}$ data table with its contributing systematic uncertainties are appended in Appendix B.1.

For the combined fit the structure functions $F_{2}$ and $x F_{3}$ were simultaneously extracted from the differential cross section using the differential cross section covariance matrix as described in Sec 3.6.2). As a result, all information about the contributing systematic uncertainties is inglobed into one single error. These data tables are provided in Appendix B.2. 


\subsubsection{Non-Singlet Fit Results}

Table 4.1 shows non-singlet fit results using only statistical errors. The results are presented for both $\overline{M S}$ and $A C O T$ models. We notice that the inclusion of the quark masses into calculations ( $A C O T$ model) causes a slight increase in the value for $\Lambda_{Q C D}$.

Table 4.1: Non-Singlet QCD fit results with statistical errors only.

\begin{tabular}{|l||c|c|}
\hline \hline Parameter & $\overline{M S}$ & ACOT \\
\hline \hline$\Lambda^{\left(n_{f}=4\right)}(\mathrm{MeV})$ & $442 \pm 58$ & $454 \pm 57$ \\
\hline$A 1_{u_{v}}$ & $0.73 \pm 0.01$ & $0.73 \pm 0.01$ \\
$A 2_{u_{v}}$ & $3.54 \pm 0.05$ & $3.53 \pm 0.05$ \\
$A 0_{u_{v}}+A 0_{d_{v}}$ & $4.79+2.39$ & $4.77+2.38$ \\
$\chi^{2} / d_{o f}$ & $97 / 59$ & $93 / 59$ \\
\hline \hline
\end{tabular}

Table 4.2 shows fit results using the structure function covariance matrix that takes into account statistical and systematic errors. We observe that $\Lambda_{Q C D}$ increases by $\approx 35$ units

Table 4.2: Non-Singlet QCD fit results with all systematic errors.

\begin{tabular}{|l||c|c|}
\hline \hline Parameter & $\overline{M S}$ & ACOT \\
\hline \hline$\Lambda^{\left(n_{f}=4\right)}(\mathrm{MeV})$ & $476 \pm 60$ & $488 \pm 59$ \\
\hline$A 1_{u_{v}}$ & $0.73 \pm 0.01$ & $0.73 \pm 0.01$ \\
$A 2_{u_{v}}$ & $3.47 \pm 0.06$ & $3.47 \pm 0.06$ \\
$A 0_{u_{v}}+A 0_{d_{v}}$ & $4.74+2.37$ & $4.73+2.36$ \\
$\chi^{2} / d o f$ & $78 / 59$ & $77 / 59$ \\
\hline \hline
\end{tabular}

compared to the previous fit, while the goodness of the fit is improved, i.e. $\chi^{2} /$ dof decreases to $77 / 59$ (with a probability of $5 \%$ ). We also note that the overall error for $\Lambda_{Q C D}$ has changed 
little. Hence, there are cancellation effects among the systematic errors. This also might be emphasized by the fact that $x F_{3}$ is determined from the difference of the antineutrino to neutrino cross sections. This method of evaluating the $\Lambda_{Q C D}$ and its error does not provides us information of the weight of each systematic. The effects of each contributing systematic is discussed in Section 4.2.5.

Figure 4.2 shows the non-singlet fit results as function of $\log Q^{2}$ in all $x$ bins using both theory models. The QCD NLO curves fit well the NuTeV data. The two theory models are nearly indistinguishable. Scaling violations are clearly seen in the deviations from flatness of the curves for different $x$ bins.

\subsubsection{Combined $F_{2}$ and $x F_{3}$ Fit Results}

The combined fit results using only statistical errors are shown in Table 4.3 for both $\overline{M S}$ and ACOT models. We observe that the inclusion of quark masses increases the value of $\Lambda_{Q C D}$

Table 4.4 shows the combined fit results which include systematic uncertainties via differential cross section error matrix. Similarly to the non-singlet case, we observe that $\Lambda_{Q C D}$ increases when we take into account the systematic errors (by $\approx 25$ units). Also, the $\chi^{2}$ decreases when $F_{2}$ and $x F_{3}$ are simultaneously extracted using the differential cross section covariance matrix, because the errors increase by a factor $\approx 3$ compared to the case when only statistical errors are used. This increase causes $\chi^{2}$ to decrease by a factor $\approx 9$ when the systematic errors are taken into account $\left(\chi^{2} \propto 1 / \sigma^{2}\right)$. Also, the uncertainty on $\Lambda_{Q C D}$ increases by a factor two when the systematic uncertainties are taken into account. We speculate that $F_{2}$ dominates, being determined from the sum of neutrino and anti-neutrino cross sections.

Figures 4.3, 4.4 show the NLO QCD fit results for $F_{2}$ and $x F_{3}$ as function of $\log Q^{2}$ in all $x$ bins. Both models fit well NuTeV data (with statistical and systematic uncertainties). 


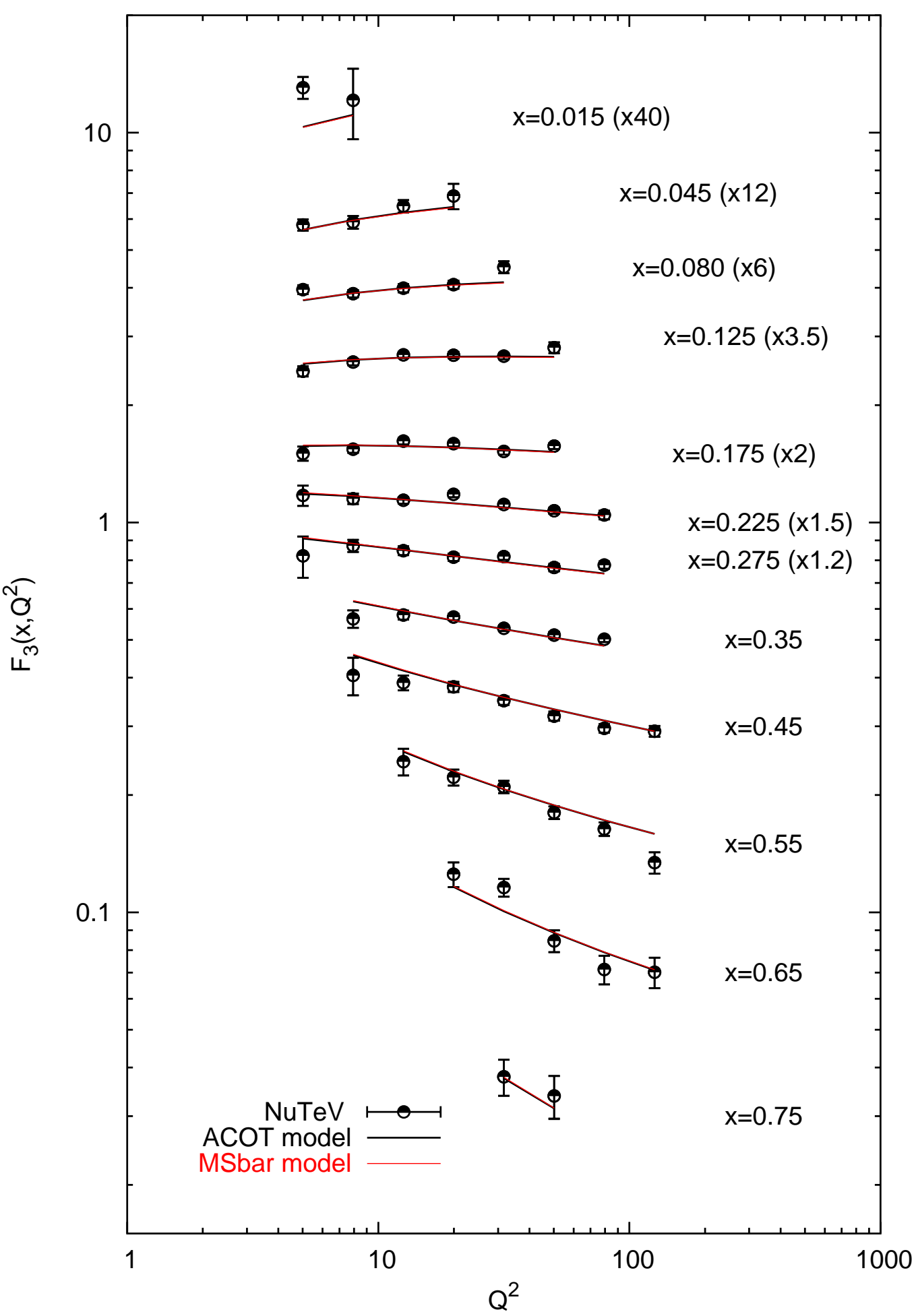

Figure 4.2: $x F_{3}$ non-singlet fit using $A C O T$ and $\overline{M S}$ models. Points are NuTeV data, curves show fits. Error bars contain both statistical and systematical uncertainties. 
Table 4.3: Combined QCD fit results; only statistical errors.

\begin{tabular}{|l||c|c|}
\hline \hline Parameter & $\overline{M S}$ & ACOT \\
\hline \hline$\Lambda^{\left(n_{f}=4\right)}(\mathrm{MeV})$ & $410 \pm 23$ & $434 \pm 22$ \\
\hline$A 1_{u_{v}}$ & $0.74 \pm 0.01$ & $0.75 \pm 0.01$ \\
$A 2_{u_{v}}$ & $3.55 \pm 0.02$ & $3.56 \pm 0.02$ \\
$A 0_{u_{v}}+A 0_{d_{v}}$ & $4.88+2.44$ & $4.97+2.49$ \\
$A 0_{u d}$ & $0.71 \pm 0.01$ & $0.71 \pm 0.01$ \\
$A 2_{u d}$ & $6.89 \pm 0.08$ & $7.06 \pm 0.08$ \\
$A 0_{g}$ & 2.35 & 2.09 \\
$A 2_{g}$ & $4.88 \pm 0.64$ & $4.23 \pm 0.47$ \\
\hline$\chi^{2} / d o f$ & $562 / 125$ & $548 / 125$ \\
\hline \hline
\end{tabular}

The curves correspond to the theory models which are very similar.

\subsubsection{Comparison between Non-Singlet and Combined Fits}

In this Section we compare the results between non-singlet and combined fits. We observe that $\Lambda_{Q C D}$ obtained from both non-singlet and combined fits is consistent with each other. For the combined fit the value of $\Lambda_{Q C D}$ and the shape of the gluon distribution are strongly correlated (see Appendix F.1.2 for tables of correlation among fit parameters). The statistical precision of $\Lambda_{Q C D}$ from the combined fit improves by a factor two compared to the non-singlet fit. This is mainly due to the inclusion of $F_{2}$ data set in the combined fit. Also, the size of the errors for structure functions used in the combined fit are larger than these used in the non-singlet fit. This explains the difference in the goodness of the fit between these two types of fits. For example, we compare the $\chi^{2}$ value of non-singlet fit using data file from 
Table 4.4: Combined QCD fit results; all systematic errors included.

\begin{tabular}{|l||c|c|}
\hline \hline Parameter & $\overline{M S}$ & ACOT \\
\hline \hline$\Lambda^{\left(n_{f}=4\right)}(\mathrm{MeV})$ & $433 \pm 51$ & $458 \pm 41$ \\
\hline$A 1_{u_{v}}$ & $0.71 \pm 0.02$ & $0.72 \pm 0.02$ \\
$A 2_{u_{v}}$ & $3.50 \pm 0.05$ & $3.49 \pm 0.05$ \\
$A 0_{u_{v}}+A 0_{d_{v}}$ & $4.47+2.23$ & $4.50+2.25$ \\
$A 0_{u d}$ & $0.68 \pm 0.03$ & $0.67 \pm 0.03$ \\
$A 2_{u d}$ & $6.74 \pm 0.20$ & $6.83 \pm 0.21$ \\
$A 0_{g}$ & 2.42 & 2.21 \\
$A 2_{g}$ & $4.83 \pm 1.38$ & $4.30 \pm 0.41$ \\
\hline \hline$\chi^{2} / d o f$ & $79 / 125$ & $76 / 125$ \\
\hline \hline
\end{tabular}

1 -parameter fit, which is $\chi^{2} / d o f=97 / 59$ (statistical errors only), to $\chi^{2} / d o f=173 / 62$ from the non-singlet fit using data file from 2-parameter fit (statistical errors).

\subsection{5 $\Lambda_{Q C D}$ Systematic Uncertainties}

In this Section we discuss the effect of each contributing experimental systematic uncertainty on $\Lambda_{Q C D}$. This is determined by taking each differential cross section shifted by $\pm 1 \sigma$, reextracting the structure functions from these shifted cross sections, and determining $\Lambda_{Q C D}$ for each shift. Therefore, we consider the "effective" systematic uncertainty on $\Lambda_{Q C D}$ by

$$
\Lambda(\text { sys })=\frac{\Lambda(+)+\Lambda(-)}{2}
$$

where $\Lambda( \pm)$ denotes $\Lambda_{Q C D}$ extracted for each cross-section systematic shifted up and down by $1 \sigma$. Because most of these errors are of the size of statistical fluctuations we cannot 


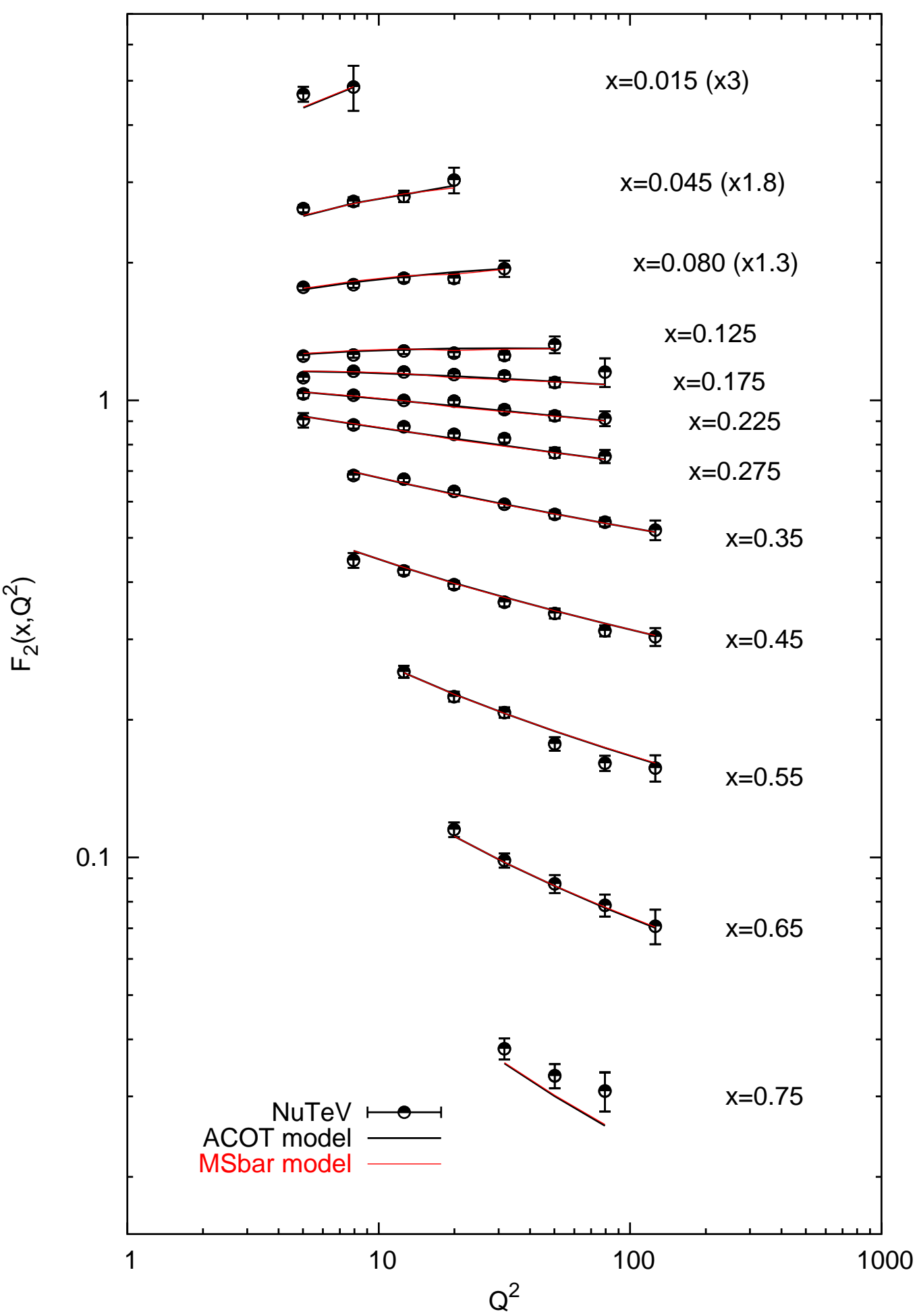

Figure 4.3: $F_{2}$ from combined fit using $A C O T$ and $\overline{M S}$ models; both statistical and systematic uncertainties included 


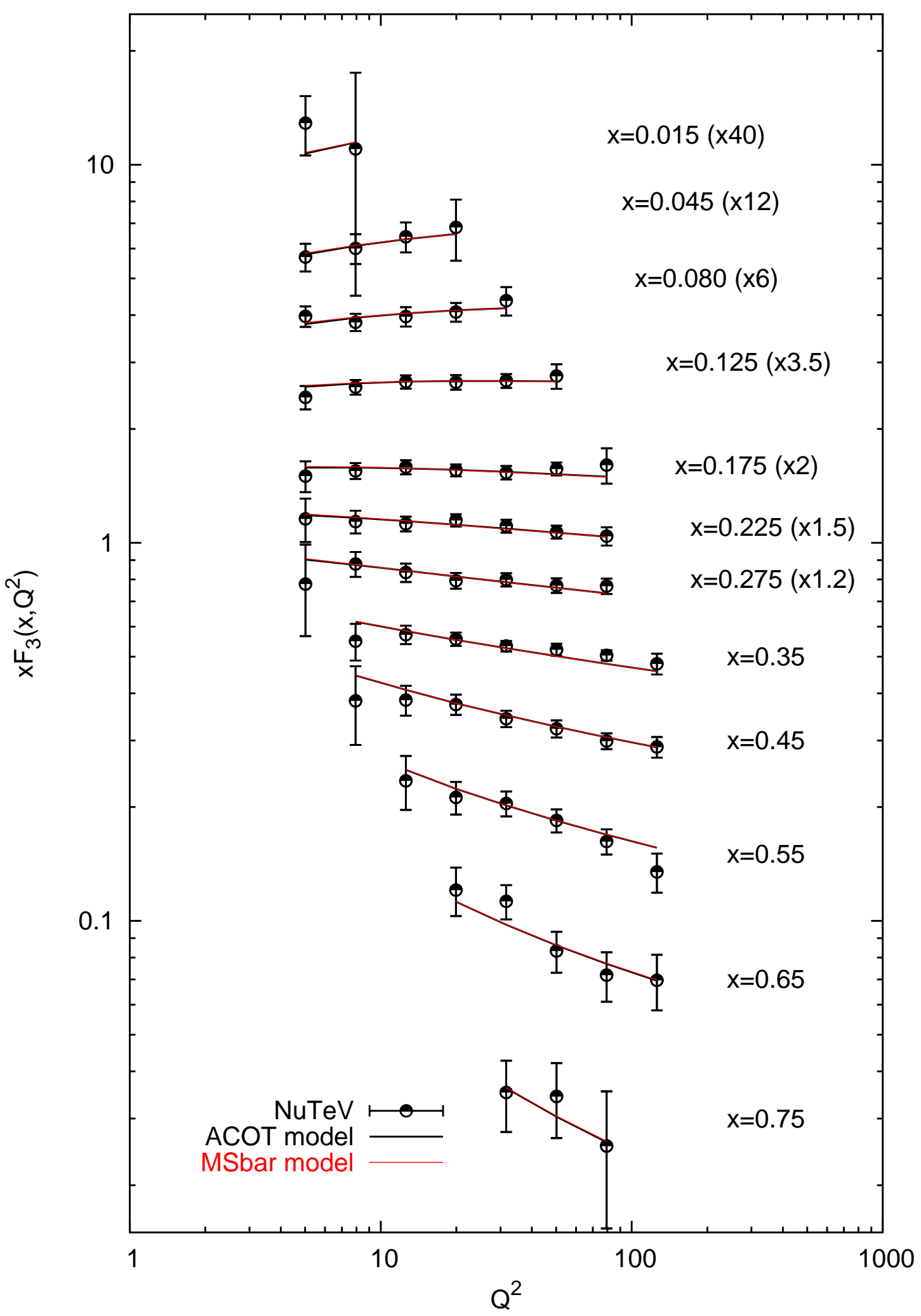

Figure 4.4: $x F_{3}$ from combined fit using $A C O T$ and $\overline{M S}$ models; both statistical and systematic uncertainties included 
accurately determine their effect. Therefore, we repeat the above procedure for $\pm 2 \sigma$ shifts of each systematic (for our default model, $A C O T$ ), whenever is possible. If a $2 \sigma$ shift gives the same order for the error we assume it is a negligible contribution. The results for nonsinglet fit are listed in the Table 4.5. The largest experimental systematic uncertainties are due to the energy scales, and the energy smearing effects in our simulation. Other experiments systematic contribution are negligible.

Table 4.5: Non-Singlet fit results showing the effect of each contributing systematic by varying each $\pm 1 \sigma, \pm 2 \sigma$.Both massless and massive predictions are shown.

\begin{tabular}{|l||c|c||c|c|c|c|}
\hline \hline & \multicolumn{1}{|c||}{$\overline{M S}$} & \multicolumn{4}{c|}{ ACOT } \\
\hline \hline$\Lambda_{\text {Central }} \pm$ stat $(\mathrm{MeV})$ & $442 \pm 58$ & \multicolumn{3}{|c|}{$454 \pm 57$} \\
\hline$\chi^{2} /$ dof & \multicolumn{2}{|c||}{$97 / 59$} & \multicolumn{3}{c|}{$93 / 59$} \\
\hline \hline Systematic & $+1 \sigma$ & $-1 \sigma$ & $+1 \sigma$ & $-1 \sigma$ & $+2 \sigma$ & $-2 \sigma$ \\
\hline \hline$E_{\mu}$ scale & +67 & -53 & +76 & -38 & +130 & -92 \\
\hline$E_{\text {had }}$ scale & -23 & +46 & -10 & +65 & -33 & +109 \\
\hline$E_{\mu}$ smear model & -37 & -28 & -66 \\
\hline$E_{\text {had }}$ smear model & -5 & -9 & +3 & 0 & -10 & -16 \\
\hline$m_{c}$ & -20 & -5 & +1 & 0 & -10 & 0 \\
\hline$\frac{B}{A}$ & -10 & -7 & -6 & +4 & -6 & +3 \\
\hline \hline
\end{tabular}

Results of the systematic error analysis for the combined fit are shown in in Table 4.6. Additional systematic contributions due to the model inputs, $\Delta x F_{3}$ and $R_{L}$ (see Sect. 3.6.3) The largest systematic errors are again due to the energy scales and the smearing models and are of the order of statistical precision. We note that the systematic errors due to the flux uncertainties, in particular charm mass (see Section 3.5), has more weight for $A C O T$ model than for $\overline{M S}$ model. 
Table 4.6: Combined QCD fit results showing the effect of each contributing systematic. Both massless and massive predictions are shown.

\begin{tabular}{|c|c|c|c|c|c|c|}
\hline & \multicolumn{2}{|c|}{$\overline{M S}$} & \multicolumn{4}{|c|}{$\mathrm{ACOT}$} \\
\hline$\Lambda_{\text {Central }}(\mathrm{MeV})$ & \multicolumn{2}{|c|}{$410 \pm 23$} & \multicolumn{4}{|c|}{$434 \pm 22$} \\
\hline$\chi^{2} / d o f$ & \multicolumn{2}{|c|}{$562 / 125$} & \multicolumn{4}{|c|}{$548 / 125$} \\
\hline Systematic & $+1 \sigma$ & $-1 \sigma$ & $+1 \sigma$ & $-1 \sigma$ & $+2 \sigma$ & $-2 \sigma$ \\
\hline$E_{\mu}$ scale & +15 & -33 & +21 & -8 & +38 & -5 \\
\hline$E_{\text {had }}$ scale & -19 & +13 & -10 & +26 & -19 & +51 \\
\hline$E_{\mu}$ smear model & \multicolumn{2}{|c|}{-23} & \multicolumn{2}{|c|}{-22} & \multicolumn{2}{|c|}{-35} \\
\hline$E_{\text {had }}$ smear model & -16 & +12 & -13 & +8 & -9 & +39 \\
\hline$m_{c}$ & +1 & -15 & +28 & -15 & +18 & -12 \\
\hline$\frac{B}{A}$ & +14 & -16 & +2 & -11 & +39 & -20 \\
\hline$R_{W}$ & +12 & -12 & +8 & -13 & +16 & -27 \\
\hline$\Delta x F_{3}$ model & \multicolumn{2}{|c|}{+25} & \multicolumn{2}{|c|}{+31} & \multicolumn{2}{|c|}{-} \\
\hline
\end{tabular}

\subsubsection{Theoretical Uncertainties}

A hard scattering calculation introduces a renormalization scale to remove the ultraviolet divergences (see Sect.1.3.1.1), and a factorization scale that separates the hard from soft physics (see Sect. 1.3.1.5). The scale dependence which arises represents the main theoretical uncertainty in the NLO DIS measurements of $\Lambda_{Q C D}$. We also evaluate the systematic uncertainties associated with quark masses. These are found to have small effects.

\subsubsection{Scale Dependence}

Factorization and renormalization scales $\left(\mu_{F}, \mu_{R}\right.$, respectively) are both usually chosen to 
be $Q$, the characteristic energy scale in the process. In this standard convention various logarithms in the calculations vanish. This program is using $\mu_{R}=\mu_{F}=Q$ as well, and thus does not include the vanishing logs explicitly in the program. In order to properly account for the scale dependence, these contributions need to be re-introduced in code.

The scale dependencies appear in the general form for structure functions as follows:

$$
F\left(x, Q^{2}\right)=\sum_{i} C_{i}\left(\mu_{R}, \mu_{F}, Q^{2}, y\right) \otimes f_{i}\left(z, \mu_{F}\right)
$$

where $C_{i}\left(\mu_{R}, \mu_{F}, Q^{2}, y\right)$ are the coefficient functions that depend both on renormalization and factorization scales, and $f_{i}\left(z, \mu_{F}\right)$ are the PDFs which depend on the factorization scale. The physical structure functions should not depend on the choices made for $\mu_{F}$ or $\mu_{R}$. If calculations could be carried to infinite order in the perturbation series, the scale dependencies would cancel out, but for a truncated order calculation, scale dependencies remain. The calculations in this analysis are performed to NLO in $\alpha_{S}$, therefore

$$
\begin{aligned}
& \mu_{R} \frac{\partial F}{\partial \mu_{R}}=0 \text { up to } \mathrm{O}\left(\alpha_{S}^{2}\right) \\
& \mu_{F} \frac{\partial F}{\partial \mu_{F}}=0 \text { up to } \mathrm{O}\left(\alpha_{S}^{2}\right)
\end{aligned}
$$

The general form for the NLO structure function, $F\left(x, Q^{2}\right)$ can be written by expanding the hard scattering coefficients up to NLO as follows:

$$
F\left(x, Q^{2}\right)=\sum_{i}\left[C_{i}^{0}\left(\mu_{R}, \mu_{F}, Q^{2}, y\right)+\alpha_{S}\left(\mu_{R}\right) C_{i}^{1}\left(\mu_{R}, \mu_{F}, Q^{2}, y\right)\right] \otimes f_{i}\left(z, \mu_{F}\right)
$$

Then,

$$
\mu_{R} \frac{\partial F}{\partial \mu_{R}}=\sum_{i}[\underbrace{\mu_{R} \frac{\partial C_{i}^{0}}{\partial \mu_{R}}}_{O\left(\alpha_{s}^{0}\right)}+\underbrace{\mu_{R} \frac{\partial \alpha_{S}}{\partial \mu_{R}} C_{i}^{1}}_{O\left(\alpha_{s}^{2}\right)}+\underbrace{\alpha_{S} \frac{\partial C_{i}^{1}}{\partial \mu_{R}}}_{O\left(\alpha_{s}^{1}\right)}] \otimes f_{i}=0 \Longrightarrow \alpha_{S} \frac{\partial C_{i}^{1}}{\partial \mu_{R}}=0
$$

where the $C_{i}^{0}$ has no $\mu_{R}$ dependence. This means that $\mu_{R}$ enters in the NLO term of the coefficient functions, but at this order there are no compensating $\mu_{R}$ terms to cancel it out. 
Performing a similar analysis for the factorization scale using Eq. (4.31), one obtains canceling terms in the coefficient functions $C_{i}^{1}$, i.e. $\frac{C_{q}^{0}}{2 \pi} P_{q q}\left(P_{q g}\right) \ln \frac{Q^{2}}{\mu_{F}^{2}}$. In these logarithmic terms, for $\mu_{F}=Q$ cancellations occur up to $\mathcal{O}\left(\alpha_{\mathrm{S}}^{2}\right)$, but not for an arbitrary choice of $\mu_{F}$. The method of evaluating the scale dependence is by its nature imprecise due to the subjectivity of defining the reasonable range over which the scale is varied. Currently there is no preferred method to evaluate the theoretical uncertainty due to scale dependence in finite order calculations. To get a range of uncertainty the choice of factorization scale is varied up and down by a factor of two (conform Ref. [4]), while the renormalization scale is kept to $\mu_{R}=Q$. The results of this study are shown in Table 4.7 for the $\overline{M S}$ model (results should be similar for $A C O T$ model).

Table 4.7: Factorization scale dependence for $\overline{M S}$ model.

\begin{tabular}{|c||c|c||c|c|}
\hline \hline \multicolumn{1}{|c||}{} & \multicolumn{2}{|c||}{$x F_{3}$ fit } & \multicolumn{2}{c|}{$x F_{3}$ and $F_{2}$ fit } \\
$C_{i}$ & $\Lambda^{\left(n_{f}=4\right)}(\mathrm{MeV})$ & $\chi^{2} / d o f$ & $\Lambda^{\left(n_{f}=4\right)}(\mathrm{MeV})$ & $\chi^{2} / d o f$ \\
\hline \hline 1 & $476 \pm 60$ & $78 / 59$ & $433 \pm 51$ & $79 / 125$ \\
\hline 2 & +74 & $79 / 59$ & +61 & $75 / 125$ \\
0.5 & -113 & $53 / 44$ & -87 & $47 / 93$ \\
\hline
\end{tabular}

We evaluate a theoretical uncertainty for $\Lambda_{Q C D}$ due to the factorization scale dependence to be ${ }_{-113}^{+74} \mathrm{MeV}$ for the non-singlet fit. For the combined fit, the theoretical uncertainty for $\Lambda_{Q C D}$ due to the factorization scale dependence is estimated to be ${ }_{-87}^{+61} \mathrm{MeV}$. Since this is the dominant uncertainty in the measurement one expects that precision will improve with improvement in theoretical predictions.

\subsubsection{Quark Masses Dependence}

The quark mass dependence could arise in the massless model ( $\overline{M S}$ model $)$ only at the 
matching conditions in $\alpha_{S}$ when a heavy quark threshold is crossed. The variation of the charm and bottom quark masses could introduce small errors. We consider effects due to charm and bottom quark masses separately.

- Charm mass uncertainty: For $Q^{2}>5 \mathrm{GeV}^{2}$ we are above the threshold for which we would consider only three number of active flavors, $n_{f}=3$. Therefore, there is no charm mass dependence for the $\overline{M S}$ model. For the $A C O T$ model, which explicitly takes into account the quark masses, we expect to see some dependence, especially for the combined fit ( $F_{2}$ is sensitive to charm production). The effects of varying the quark mass by $\pm 1 \sigma$ are shown in Table 4.8. Negligible effects on $\Lambda_{Q C D}$ are observed for non-singlet fit. The effects for combined fit are small. Even if we shift the quark masses by a factor of two the effects are still negligible for non-singlet fit, and small for combined fit.

Table 4.8: The effect of $m_{c}$ varied by $\pm 1 \sigma$ and by a factor two on $\Lambda_{Q C D}$, for both non-singlet and combined fits.

\begin{tabular}{|l|l||c|c|c|c|}
\hline \hline & \multicolumn{1}{|c||}{} & \multicolumn{2}{c|}{$x F_{3}$} & \multicolumn{2}{c|}{$F_{2}+x F_{3}$} \\
Model & $m_{c}(\mathrm{GeV})$ & $\Lambda(\mathrm{MeV})$ & $\chi^{2} / d o f$ & $\Lambda(\mathrm{MeV})$ & $\chi^{2} / d o f$ \\
\hline ACOT & 1.4 & $488 \pm 59$ & $77 / 59$ & $458 \pm 41$ & $76 / 125$ \\
ACOT & $-1 \sigma$ & -4 & $77 / 59$ & -2 & $76 / 125$ \\
ACOT & $+1 \sigma$ & +2 & $77 / 59$ & +10 & $76 / 125$ \\
\hline ACOT & 0.7 & -2 & $77 / 59$ & -6 & $77 / 125$ \\
ACOT & 2.8 & +2 & $77 / 59$ & +18 & $80 / 125$ \\
\hline \hline
\end{tabular}

- Bottom mass uncertainty: Both models should see some dependence on the uncertainty in the bottom quark mass. Table 4.9 shows the result of varying the b-threshold within $1 \sigma$ of its central value. The effects are negligible for both theory models.

We could also assess the theoretical uncertainty due to quark mass effects by looking at 
Table 4.9: The effect of varying the bottom mass by $\pm 1 \sigma$ for non-singlet and combined fits. The results are shown for both theory models.

\begin{tabular}{|l|l||c|c|c|c|}
\hline \hline \multirow{2}{*}{} & \multicolumn{1}{|c||}{} & \multicolumn{2}{c|}{$x F_{3}$} & \multicolumn{2}{c|}{$F_{2}+x F_{3}$} \\
Model & $m_{b}(\mathrm{GeV})$ & $\Lambda(\mathrm{MeV})$ & $\chi^{2} / d o f$ & $\Lambda(\mathrm{MeV})$ & $\chi^{2} / d o f$ \\
\hline$\overline{M S}$ & 4.3 & $476 \pm 60$ & $78 / 59$ & $433 \pm 51$ & $79 / 125$ \\
$\overline{M S}$ & 4.1 & +5 & $78 / 59$ & -5 & $79 / 125$ \\
$\overline{M S}$ & 4.5 & +1 & $78 / 59$ & +6 & $77 / 125$ \\
\hline ACOT & 4.3 & $488 \pm 59$ & $77 / 59$ & $458 \pm 41$ & $76 / 125$ \\
ACOT & 4.1 & +1 & $76 / 59$ & -17 & $77 / 125$ \\
ACOT & 4.5 & +0 & $77 / 59$ & -12 & $76 / 125$ \\
\hline \hline
\end{tabular}

the difference between $A C O T$ and $\overline{M S}$ models. ACOT is a model that fully accounts for quark masses, while $\overline{M S}$ model assumes massless quarks. The difference in the value of $\Lambda_{Q C D}$ for non-singlet fit is $+12 \mathrm{MeV}$ corresponding to a difference in $\alpha_{S}\left(M_{Z}\right)$ of +0.0003 . For the combined fit, the difference in the value of $\Lambda_{Q C D}$ is of $+25 \mathrm{MeV}$, which corresponds to a positive shift of +0.0011 in $\alpha_{S}\left(M_{Z}\right)$ value. The effects of the quark mass are small compared to the scale uncertainty.

\subsubsection{Relate $\Lambda_{Q C D}$ to $\alpha_{S}$}

$\Lambda_{Q C D}$ provides a parametrization of the scale dependence of $\alpha_{S}$. Using Equation (1.58) we can relate our measurement of $\Lambda_{Q C D}$ to the appropriate $\alpha_{S}$, which corresponds to 2-loop beta function expansion. Furthermore, in order to compare the values of $\alpha_{S}$ from various experiments, we must evolve them to a common scale. The standard choice for this reference scale is the mass of the neutral $Z$ boson, $M_{Z}$. We obtained the following values for $\alpha_{S}\left(M_{Z}\right)$ 
using ACOT model

$$
\begin{aligned}
& \text { NS fit: } \Lambda_{A C O T}^{n_{f}=4}=488 \pm \underbrace{59}_{\exp } \underbrace{+74}_{\text {th }} \underbrace{+113} \mathrm{MeV} \rightarrow \alpha_{S}\left(M_{Z}\right)=0.1260 \pm 0.0028 \underbrace{+0.0034}_{\text {theory }} \\
& \text { CO fit: } \Lambda_{A C O T}^{n_{f}=4}=458 \pm \underbrace{41}_{\exp } \underbrace{+617}_{\text {th }} \mathrm{MeV} \rightarrow \alpha_{S}\left(M_{Z}\right)=0.1247 \pm 0.002 \underbrace{+0.00047}_{\text {theory }}
\end{aligned}
$$

where the first line corresponds to results from non-singlet fit, and the second line to results from combined fit.

\subsection{RESULT BIAS STUDY}

In this section we examine possible effects on the QCD results due to the various choices of PDF parametrization and kinematic cut dependencies. The effects of the target mass correction are also studied here. Various checks of the QCD code were performed which are presented in the Appendix G.

\subsubsection{PDF Parametrization}

Since it is possible that the choice of the PDF functional form could bias the QCD fit, we consider first a general form. For the non-singlet quark distributions the following general initial parametrization was used:

$$
x q^{N S}=x u_{v}+x d_{v}=\left(A 0_{u_{v}}+A 0_{d_{v}}\right) x^{A 1_{u_{v}}}(1-x)^{A 2_{u_{v}}} e^{A 3_{u_{v}} x}(1+x)^{A 5_{u_{v}}} .
$$

For singlet and gluon distributions the starting general forms are given by

$$
\begin{aligned}
x q^{S} & =\underbrace{x u_{v}+x d_{v}}_{x q^{N S}}+2 A 0_{u d}(1-x)^{A 2_{u d}} e^{A 3_{u d} x}(1+x)^{A 5_{u d}}, \\
x G & =A 0_{g}(1-x)^{A 2_{g}} e^{A 3_{g} x}(1+x)^{A 5_{g}} .
\end{aligned}
$$


The fit results using these forms are shown in Tables 4.11, 4.10. We observe that the parameters $A 3_{u_{v}}, A 3_{u d}, A 3_{g}, A 5_{u_{v}}, A 5_{u d}$, and $A_{g}$ are not well constrained by the fit regardless of what theory model is used. Therefore, a simplified functional forms for the non-singlet, singlet and gluon distributions are adopted, as

$$
\begin{aligned}
x q^{N S} & =x u_{v}+x d_{v}=\left(A 0_{u_{v}}+A 0_{d_{v}}\right) x^{A 1_{u_{v}}}(1-x)^{A 2_{u_{v}}}, \\
x q^{S} & =\underbrace{x u_{v}+x d_{v}}_{x q^{N S}}+2 A 0_{u d}(1-x)^{A 2_{u d}}, \\
x G & =A 0_{g}(1-x)^{A 2_{g}} .
\end{aligned}
$$

Other PDF functional forms have been tried for both non-singlet and combined fits.

\subsubsection{Kinematic Cut Dependence: $x$}

$\mathrm{NuTeV}$ data ranges up to $x=0.75$ where it is possible that Fermi motion effects are important. To understand how these may affect QCD fits, Table 4.12 shows the effect of removing the largest $x$ bin on the measurement of $\Lambda_{Q C D}$ for both non-singlet and combined fits. We observe no significant dependence on including the highest $x$ bin.

\subsubsection{Kinematic Cut Dependence: $Q^{2}$}

As discussed in section 1.3.4, at low $Q^{2}$, non-perturbative effects become important. Here we explore the possible effects on $\Lambda_{Q C D}$ due to the choice of minimum $Q^{2}$ in the fits. For this study we vary $Q_{\min }^{2}$ to be $5,7,10 \mathrm{GeV}^{2}$ and use in the fit only data points with $Q^{2}>Q_{\min }^{2}$. The results of this study are shown in Table 4.13. For the combined fit we see no effect if we increase the $Q^{2}$ cut, while for the non-singlet fit we notice a small increase in $\Lambda_{Q C D}$ which is well within the uncertainty. Therefore, for the final results we choose $Q^{2}>5 \mathrm{GeV}^{2}$. 
Table 4.10: QCD fit results using $\overline{M S}$ model for the combined fit using a general and a simplified parametrization of the PDFs.

\begin{tabular}{|l||c|c|}
\hline \hline Parameters & General & Simple \\
\hline \hline$\Lambda^{\left(n_{f}=4\right)}(\mathrm{MeV})$ & $383 \pm 55$ & $433 \pm 51$ \\
\hline$A 0_{u_{v}}+A 0_{d_{v}}$ & $4.13+2.06$ & $4.47+2.23$ \\
$A 1_{u_{v}}$ & $0.70 \pm 0.11$ & $0.71 \pm 0.02$ \\
$A 2_{u_{v}}$ & $2.53 \pm .0 .75$ & $3.50 \pm 0.05$ \\
$A 3_{u_{v}}$ & $-5.55 \pm 4.75$ & - \\
$A 5_{u_{v}}$ & $5.33 \pm 5.39$ & - \\
$A 0_{u d}$ & $0.69 \pm 0.09$ & $0.68 \pm 0.03$ \\
$A 2_{u d}$ & $11.40 \pm 22.90$ & $6.74 \pm 0.20$ \\
$A 3_{u d}$ & $11.39 \pm 61.77$ & - \\
$A 5_{u d}$ & $-6.54 \pm 40.96$ & - \\
$A 0_{g}$ & 1.39 & 2.42 \\
$A 2_{g}$ & $-4 . \pm 169$ & $4.83 \pm 1.38$ \\
$A 3_{g}$ & $-93 \pm 446$ & - \\
$A 5_{g}$ & $96 \pm 287$ & - \\
\hline$\chi^{2} / d o f$ & 54119 & $79 / 125$ \\
\hline \hline
\end{tabular}

\subsubsection{Effects of Target Mass Correction}

Data includes the target mass effects, therefore we need to correct the theory predictions for these effects using Eqs. (1.80), (1.81). The target mass correction (TMC) affects the highest $x$ and low $Q^{2}$ region. We expect to see that including TMC would decrease the value of $\Lambda_{Q C D}$. Table 4.15 shows that the effect of taking into account the TMC on $\Lambda_{Q C D}$ extracted 
Table 4.11: QCD fit results using ACOT model for the combined fit using a general and a simplified parametrization of the PDFs.

\begin{tabular}{|l||c|c|}
\hline \hline Parameters & General & Simple \\
\hline \hline$\Lambda^{\left(n_{f}=4\right)}(\mathrm{MeV})$ & $462 \pm 53$ & $458 \pm 41$ \\
\hline$A 0_{u_{v}}+A 0_{d_{v}}$ & $4.07+2.04$ & $4.50 \pm 2.25$ \\
$A 1_{u_{v}}$ & $0.70 \pm 0.07$ & $0.72 \pm 0.02$ \\
$A 2_{u_{v}}$ & $2.30 \pm .0 .48$ & $3.49 \pm 0.05$ \\
$A 3_{u_{v}}$ & $-6.8 \pm 2.9$ & - \\
$A 5_{u_{v}}$ & $6.58 \pm 3.3$ & - \\
$A 0_{u d}$ & $0.71 \pm 0.05$ & $0.67 \pm 0.03$ \\
$A 2_{u d}$ & $11.27 \pm 5.3$ & $6.83 \pm 0.21$ \\
$A 3_{u d}$ & $16.52 \pm 12$ & - \\
$A 5_{u d}$ & $-13.08 \pm 7.8$ & - \\
$A 0_{g}$ & 1.57 & 2.21 \\
$A 2_{g}$ & $6.32 \pm 0.83$ & $4.30 \pm 0.41$ \\
$A 3_{g}$ & $0.12 \pm 6.07$ & - \\
$A 5_{g}$ & $4.7 \pm 6.09$ & - \\
\hline$\chi^{2} / d o f$ & & $76 / 125$ \\
\hline \hline
\end{tabular}

using both models is very small. In order to see the right trend of the TMC, the size of the correction is artificially increased by introducing a multiplicative constant $C_{i}$ in the definition of the $\xi$ variable:

$$
\xi=\frac{2 x}{1+\sqrt{1+\frac{4 m_{p}^{2} x^{2}}{Q^{2}}}} \Rightarrow \xi=\frac{2 x}{1+\sqrt{1+\frac{C_{i} 4 m_{p}^{2} x^{2}}{Q^{2}}}} .
$$


Table 4.12: The effect of removing the $x=0.75$ bin in the NLO QCD fits using both massless and massive predictions.

\begin{tabular}{|c|c||c|c||c|c|}
\hline \hline \multirow{2}{*}{ Scheme } & \multicolumn{1}{|c||}{$x_{\max }$} & $\Lambda^{\left(n_{f}=4\right)}(\mathrm{MeV})$ & $\chi^{2} / d o f$ & $\Lambda^{\left(n_{f}=4\right)}(\mathrm{MeV})$ & $\chi^{2} / d o f$ \\
\hline \hline$\overline{M S}$ & 0.75 & $476 \pm 60$ & $78 / 59$ & $433 \pm 51$ & $79 / 125$ \\
$\overline{M S}$ & 0.65 & $466 \pm 62$ & $76 / 57$ & $444 \pm 60$ & $70 / 119$ \\
\hline \hline ACOT & 0.75 & $488 \pm 59$ & $77 / 59$ & $458 \pm 41$ & $76 / 125$ \\
ACOT & 0.65 & $477 \pm 60$ & $75 / 57$ & $435 \pm 40$ & $65 / 119$ \\
\hline \hline
\end{tabular}

Table 4.13: The effect of the increasing $Q^{2}$ cut on the extracted $\Lambda_{Q C D}$

\begin{tabular}{|c|c||c|c||c|c|}
\hline \hline & \multicolumn{1}{|c||}{} & & \multicolumn{2}{c||}{$x F_{3}$ fit } & \multicolumn{2}{|c|}{$x F_{3}$ and $F_{2}$ fit } \\
Scheme & $Q_{\text {min }}^{2}$ & $\Lambda^{\left(n_{f}=4\right)}(\mathrm{MeV})$ & $\chi^{2} / d o f$ & $\Lambda^{\left(n_{f}=4\right)}(\mathrm{MeV})$ & $\chi^{2} / d o f$ \\
\hline \hline$\overline{M S}$ & 5 & $476 \pm 60$ & $78 / 59$ & $433 \pm 51$ & $79 / 125$ \\
$\overline{M S}$ & 7 & $522 \pm 70$ & $62 / 52$ & $406 \pm 47$ & $63 / 111$ \\
$\overline{M S}$ & 10 & $525 \pm 82$ & $52 / 43$ & $434 \pm 48$ & $48 / 93$ \\
\hline $\mathrm{ACOT}$ & 5 & $488 \pm 59$ & $77 / 59$ & $458 \pm 41$ & $76 / 125$ \\
$\mathrm{ACOT}$ & 7 & $535 \pm 69$ & $60 / 52$ & $421 \pm 53$ & $61 / 111$ \\
$\mathrm{ACOT}$ & 10 & $543 \pm 82$ & $52 / 43$ & $449 \pm 48$ & $48 / 93$ \\
\hline \hline
\end{tabular}

The study of varying the $C_{i}=1.5,2$ concludes that the increase in the size of the TMC decreases $\Lambda_{Q C D}$ as expected (see Table 4.14). 
Table 4.14: Artificial increase of the TMC in order to observe the direction of the effect on $\Lambda_{Q C D}$ using both theoretical models.

\begin{tabular}{|c|c|c|c|c|c|}
\hline & \multirow[b]{2}{*}{$C_{i}$} & \multicolumn{2}{|c|}{$x F_{3}$ fit } & \multicolumn{2}{|c|}{$x F_{3}$ and $F_{2}$ fit } \\
\hline & & $\Lambda^{\left(n_{f}=4\right)}(\mathrm{MeV})$ & $\chi^{2} / d o f$ & $\Lambda^{\left(n_{f}=4\right)}(\mathrm{MeV})$ & $\chi^{2} / d o f$ \\
\hline \multirow{3}{*}{$\overline{M S}$} & 1 & $476 \pm 60$ & $78 / 59$ & $433 \pm 51$ & $79 / 125$ \\
\hline & 1.5 & 426 & $77 / 59$ & 334 & $80 / 125$ \\
\hline & 2 & 359 & $77 / 59$ & 250 & $84 / 125$ \\
\hline \multirow{3}{*}{$A C O T$} & 1 & $488 \pm 59$ & $77 / 59$ & $458 \pm 41$ & $76 / 125$ \\
\hline & 1.5 & 438 & $75 / 59$ & 387 & $75 / 125$ \\
\hline & 2 & 373 & $75 / 59$ & 326 & $81 / 125$ \\
\hline
\end{tabular}

Table 4.15: Fit results using both theoretical models with and without Target Mass Correction. Results from both NS and Combined fits are shown with all the correlations errors taken into account.

\begin{tabular}{|c|c||c|c|c||c|c|c|}
\hline \hline & \multicolumn{1}{|c||}{} & \multicolumn{3}{c||}{$x F_{3}$ fit } & \multicolumn{3}{c|}{$x F_{3}$ and $F_{2}$ fit } \\
TMC & scheme & $\Lambda^{\left(n_{f}=4\right)}(\mathrm{MeV})$ & $\chi^{2} / d o f$ & Prob $\%$ & $\Lambda^{\left(n_{f}=4\right)}(\mathrm{MeV})$ & $\chi^{2} /$ dof & Prob $\%$ \\
\hline \hline $\mathrm{ON}$ & $\overline{M S}$ & $476 \pm 60$ & $78 / 59$ & 5.9 & $439 \pm 54$ & $79 / 125$ & 99.9 \\
$\mathrm{OFF}$ & $\overline{M S}$ & +31 & $87 / 59$ & 1.0 & -4 & $93 / 125$ & 98.5 \\
\hline $\mathrm{ON}$ & \multirow{2}{*}{ ACOT } & $488 \pm 59$ & $77 / 59$ & 5.8 & $458 \pm 41$ & $76 / 125$ & 99.9 \\
$\mathrm{OFF}$ & \multirow{2}{*}{ ACOT } & +14 & $86 / 59$ & 1.2 & +2 & $90 / 125$ & 99.2 \\
\hline \hline
\end{tabular}




\subsection{COMPARISONS OF NUTEV $\alpha_{S}$ TO OTHER MEASUREMENTS}

In this section we compare our measurement of $\alpha_{S}$ to results from other experiments. All the measurements are evolved to the reference scale, $M_{Z}$. Figure 4.5 shows the results of $\alpha_{S}\left(M_{Z}\right)$ from various experiments [4]. The green band denotes the world weighted average $\alpha_{S}\left(M_{Z}\right)=0.1185 \pm 0.0020$, as quoted by Particle Data Group 2005 (using selected measurements), which excludes the Lattice QCD calculations. If the Lattice QCD result is included in the global fit, then the world average for $\alpha_{S}\left(M_{Z}\right)$ is decreased to $\alpha_{S}\left(M_{Z}\right)=0.1176 \pm 0.0020$. Both $\mathrm{NuTeV}$ measurements are shown in red (from combined and non-singlet fits). Both results situate above the world average, but are consistent with the world average value. Our results are in better agreement with experiments of higher characteristic energy scales, such as $e^{+} e^{-}$collisions, which favor a higher average $\alpha_{S}\left(M_{Z}\right)$ This reduces any potential discrepancies between low and high $Q^{2}$ measurements observed by previous low $Q^{2}$ average values (described below). In the next sections we summarize the results from various experiments used in this comparison.

\subsection{1 $\alpha_{S}$ from DIS}

As discussed in Section 1.2, DIS provided one of the first precise methods of determining $\alpha_{S}$ from NLO QCD fits to scaling violating structure functions. The combined analysis of SLAC/BCDMS data $[44,67,68]$ in a $Q^{2}$ range from 0.5 to $260 \mathrm{GeV}^{2}$ produced $\alpha_{S}\left(M_{Z}\right)=$ $0.113 \pm 0.005$, where all the errors (experimental and theoretical) are included [57]. The small value for strong coupling from DIS measurements caused some speculations in explaining the differences from measurements in $e^{+} e^{-}$processes. A more recent reanalysis of the SLAC, BCDMS and NMC data on $F_{2}$ that takes proper account of point-to-point correlations resulted in an increase of $\alpha_{S}\left(M_{Z}\right)$ to $0.118 \pm 0.005$ [58].

The most precise previous determination of $\alpha_{S}$ from fixed-target DIS experiments is 


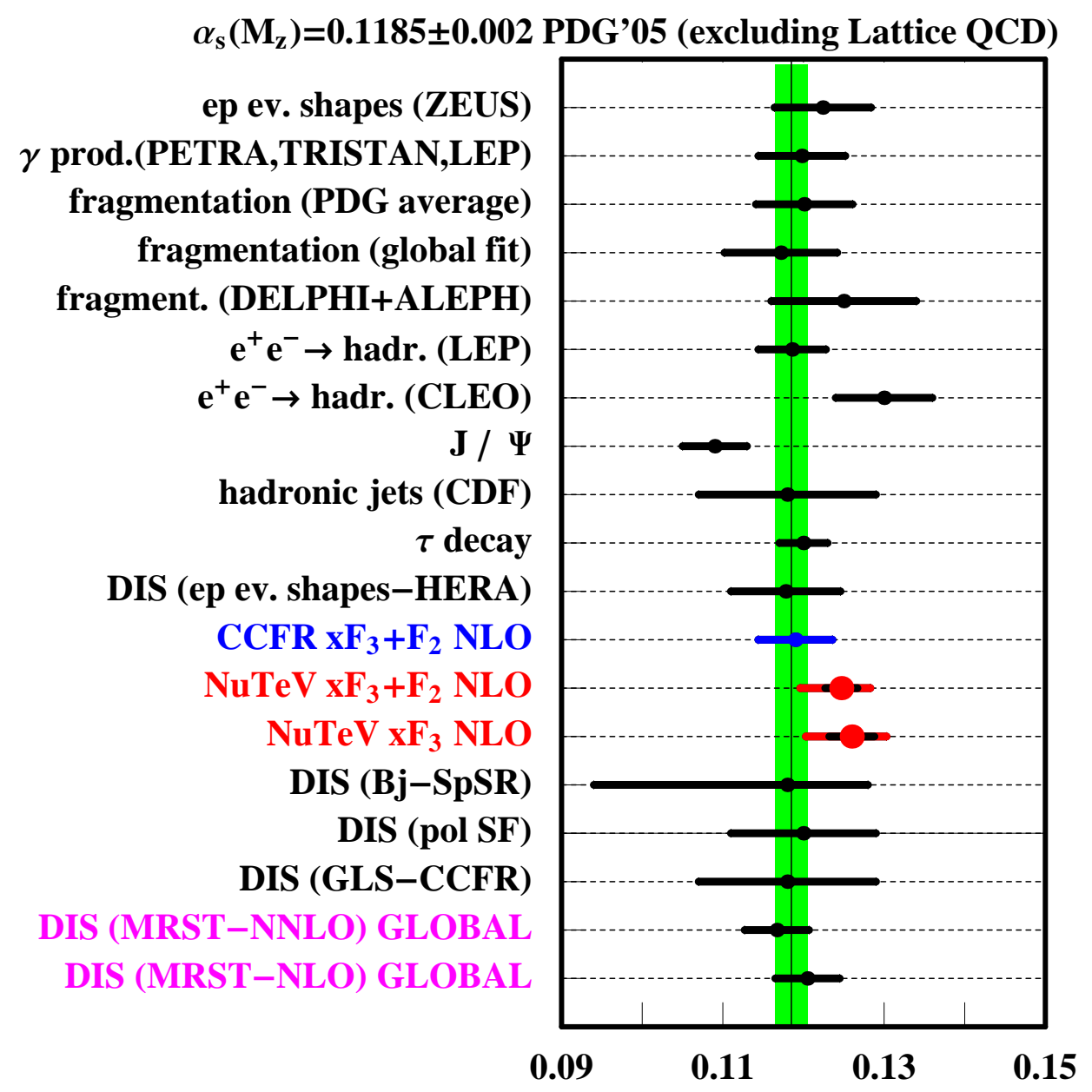

Figure 4.5: Compare $\mathrm{NuTeV} \alpha_{S}\left(M_{Z^{0}}\right)$ to other experimental measurements. The values are taken from PDG 2005. The world average excluding Lattice QCD is $\alpha_{S}\left(M_{Z}\right)=0.1185 \pm$ 0.0020 .

provided by the CCFR $\nu$-nucleon scattering experiment shown in blue in Figure 4.5, labeled as "(CCFR $\left.\mathrm{xF}_{3}+\mathrm{F}_{2} \mathrm{NLO}\right)$ "), which yielded an $\alpha_{S}\left(M_{Z}\right)=0.119 \pm 0.002 \pm 0.001(H T) \pm 0.004$ [51]. Some discussions on the CCFR comparison is presented in Section 4.4.1.1.

A global fit, which includes selected scaling violation data, was performed by the MRST04 group [59]. They find $\alpha_{S}\left(M_{Z}\right)=0.1205 \pm 0.004$ at NLO (labeled DIS(MRST NLO)), and $\alpha_{S}\left(M_{Z}\right)=0.1167 \pm 0.004$ at NNLO (labeled as "DIS(MRST NNLO)"). We are in good 
agreement with the former NLO DIS result.

Another important measurement of $\alpha_{S}$ is from a fit to Gross-Llewellyn Smith sum rule obtained by the CCFR experiment [60]. The measured $\alpha_{S}\left(M_{Z}\right)$ is known here to the NNLO, and corresponds to $\alpha_{S}\left(M_{Z}\right)=0.118 \pm 0.002$ (stat) \pm 0.005 (sys) $\pm 0.003($ th), with theoretical error dominated by the higher-twist contribution (labeled as "DIS(GLS-CCFR)").

Polarized lepton-nucleon scattering provides another determination of $\alpha_{S}$. The spindependent structure functions from SMC [61] and HERMES [62] data yield $\alpha_{S}\left(M_{Z}\right)=$ $0.120 \pm 0.009$ (labeled as "DIS(pol SF)"). The strong coupling obtained from the polarized Bjorken sum rule fit gives $\alpha_{S}\left(M_{Z}\right)=0.118_{-0.024}^{+0.010}$ (labeled as "DIS(Bj-SpSR)"). The larger error are due to the extrapolation into small $x$ region which is unmeasured. These results are shown in Figure 4.5.

$\alpha_{S}$ can also be obtained from ep scattering processes at HERA by comparing the jet rates of $(1+1)$ to $(2+1)$ processes. The $(1+1)$ jet state corresponds to the lowest order in $\alpha_{S}$ scattering process, $e+q \rightarrow e+q$. The $(2+1)$ jet state corresponds to next order in $\alpha_{S}$ where a gluon is radiated, $e+q \rightarrow e+q+g$. This measurement is performed over a wide range of $Q^{2}$, using a NLO QCD calculation. The results from jet production at HERA can be summarized with the weighted average value $\alpha_{S}\left(M_{Z}\right)=0.1178 \pm 0.0033(\exp ) \pm 0.006(t h)$ (labeled as "DIS ep ev.shapes(HERA)") [65].

$\mathrm{NuTeV}$ result is as precise as the most precise DIS determination of $\alpha_{S}$. Although the central value is higher than previous measurements, it is consistent within experimental errors with other DIS results.

\subsubsection{Comparison with CCFR Results}

The CCFR experiment is the predecessor of the $\mathrm{NuTeV}$ experiment. Our measurement is as precise as the CCFR result. NuTeV measurement of $\alpha_{S}$ is about $2 \sigma$ higher than the CCFR result. Therefore, it is important to understand the differences in the $\alpha_{S}$ results. Here we 
outline the known differences:

- CCFR analysis extracted $\Lambda_{Q C D}$ from NLO QCD fits to structure functions which were corrected for the charm mass production using a LO rescaling prescription; NuTeV data does not remove the charm mass effects.

- $\mathrm{NuTeV}$ extracted structure functions by fitting differential cross sections using full pointto-point covariance matrix. The extracted differential cross section differ, in particular at high $x$. This difference was found to be due to calibration [22].

The CCFR experiment yields a value for $\Lambda_{Q C D}$ (with its corresponding $\alpha_{S}$ ) from combined $F_{2}$ and $x F_{3}$ fits as

$$
\Lambda_{\overline{M S}}^{n_{f}=4}=337 \pm 28 \mathrm{MeV} \longrightarrow \alpha_{S}\left(M_{Z}\right)=0.119 \pm 0.002 \pm \underbrace{0.001}_{H T} \pm \underbrace{0.004}_{t h}
$$

CCFR theoretical uncertainty on $\Lambda_{Q C D}$ is not estimated, but quoted from a QCD analysis of previous DIS measurements, [57]. As for the non-singlet fit, CCFR obtains [50]

$$
\Lambda_{\overline{M S}}^{n_{f}=4}=381 \pm 53 \mathrm{MeV} .
$$

The best way to directly compare the measurements is by using the same QCD program and CCFR data set extracted through a similar mechanism as $\mathrm{NuTeV}$ structure function extraction. Unfortunately, only structure function $F_{2}$ from CCFR is available [63]. Hence, we can only compare the fit results from $F_{2}$. The results from these direct comparisons give -95 MeV difference in $\Lambda_{Q C D}$, corresponding to $2 \sigma$ disagreement between CCFR and $\mathrm{NuTeV}$. This implies that the disagreement is purely due to data differences [22]. 


\subsection{2 $\alpha_{S}$ from Other Processes}

We compare NuTeV results to $\alpha_{S}$ measurements from other QCD processes besides DIS which were included in the Figure 4.5. We quote values of $\alpha_{S}$ as quoted by PDG 2005 [4]

- $\alpha_{S}$ from decays of the $\tau$ lepton, $\tau \rightarrow \nu_{\tau}+$ hadrons. The strong coupling is determined from the ratio of the hadronic to the electronic branching ratio of the tau lepton, $R_{\tau}=$ $B_{\text {hadr }} / B_{e}$. The measured value obtained is $\alpha_{S}\left(M_{Z}\right)=0.120 \pm 0.003$ (labeled " $\tau$ decay").

- $\alpha_{S}$ obtained from the production of hadronic jets with large transverse momentum. Jet data are fitted over a large range of transverse momenta, assuming a set of structure functions. The CDF experiment obtains $\alpha_{S}\left(M_{Z}\right)=0.118 \pm 0.011$ (NLO) from hadronic jet rates and event shapes (labeled "hadronic jets(CDF)" in Figure 4.5).

- $\alpha_{S}$ from $e^{+} e^{-}$annihilation can be determined from various methods: hadronic event shapes, jet production rates, energy-energy correlation function, scaling violations of fragmentation functions, total hadronic cross section.

Strong coupling from the scaling violations of the fragmentation functions in the $e^{+} e^{-} \rightarrow$ $h+X$ process, is determined similarly to the DIS scaling violations of the structure functions method. The factorization theorem is applied to separate the hard gluon radiation from the hadronization. Experiments like ALEPH, DELPHI and OPAL [64] performed measurements of $\alpha_{S}$ at NLO, yielding an average $\alpha_{S}\left(M_{Z}\right)=0.125 \pm 0.005 \pm$ 0.008 (labeled "fragment(DELPHI+ALEPH)" in Figure 4.5.)

A determination of $\alpha_{S}$ can be obtained from the total hadronic cross section. This is the first quantity for which NNLO QCD corrections have been calculated, $R_{\gamma}=$ $\frac{\sigma\left(e^{+} e^{-} \rightarrow \text { hadrons }\right)}{\sigma\left(e^{+} e^{-} \rightarrow \mu^{+} \mu^{-}\right)}$. The PETRA, TRISTAN, LEP experiments obtained $\alpha_{S}\left(M_{Z}\right)=0.1198 \pm$ 0.0054 (labeled " $\gamma$ prod.(PETRA,TRISTA,LEP)" in Figure 4.5.)

The event shape characterizes the structure of a multihadron event by a single number. There are many measurements of $\alpha_{S}$ based on global event shape variables in the energy 
range between $22 \mathrm{GeV}$ to around $200 \mathrm{GeV}$.

- $\alpha_{S}$ can be obtained from the ratios of partial decay widths at the heavy-quark mass scale, $R_{\mu}(\Upsilon)=\frac{\Gamma(\Upsilon \rightarrow \text { hadrons })}{\Gamma\left(\Upsilon \rightarrow \mu^{+} \mu^{-}\right)}$. The available data for $J / \psi$ and $\Upsilon$ resonances is very precise, therefore the errors are theoretically dominated. The Strong coupling constant is determined to be $\alpha_{S}\left(M_{Z}\right)=0.109 \pm 0.004$. The result is shown in Figure 4.5 and is labeled as " $J / \psi "$.

- $\alpha_{S}$ from Lattice QCD calculations. Mass splitting of heavy quarkonia states can be used to determine $\alpha_{S}\left(M_{Z}\right)=0.1170 \pm 0.0012$. 


\subsection{CONCLUSIONS}

This thesis has presented a new measurement of $\Lambda_{Q C D}^{n_{f}=4}$ from NLO QCD fits to $Q^{2}$ dependence of the structure functions in the $\nu$ - nucleon DIS process. This is the first determination of $\Lambda_{Q C D}$ which uses a NLO theory model taking into account heavy quark production $(A C O T$ model [12]).

Also, this analysis has presented a new method of evaluating the systematic uncertainties for $\Lambda_{Q C D}$ by constructing a full covariance error matrix which takes into account point-topoint data correlations.

The largest systematic uncertainties in measuring $\alpha_{S}$ from previous DIS experiments were due to the energy scales. The $\mathrm{NuTeV}$ experiment was designed to perform precision QCD measurements by improving considerably its knowledge of energy scales which were determined to a precision of $0.43 \%$ for hadrons and $0.7 \%$ for muons (almost a factor of two improvement compared to previous neutrino experiment).

We obtained the following results for $\Lambda_{Q C D}^{n_{f}=4}$ with its corresponding $\alpha_{S}\left(M_{Z}\right)$

$$
\begin{aligned}
& \Lambda_{A C O T}^{N L O,(n=4)}=488 \pm 59(\text { stat }+ \text { sys })_{-113}^{+74}(\text { theory }) ; \alpha_{S}\left(M_{Z}\right)=0.1260 \pm 0.0028_{0.0050}^{+0.0034} \\
& \Lambda_{A C O T}^{N L O,(n=4)}=458 \pm 41(\text { stat }+ \text { sys })_{-87}^{+61}(\text { theory }) ; \alpha_{S}\left(M_{Z}\right)=0.1247 \pm 0.0020_{-0.0047}^{+0.0030}
\end{aligned}
$$

from non-singlet and combined fits, respectively. The first error corresponds to total experimental uncertainties (statistical and systematic). The second second error is the estimated theoretical uncertainty due to factorization scale dependence. The results from non-singlet and combined fits are consistent with one another. 
$\mathrm{NuTeV}$ measurement is higher than the world average value, but is consistent within errors. We also observe that this measurement is one of the most precise determination of $\alpha_{S}$, and as precise as the most precise measurement of $\alpha_{S}$ from DIS to date. This is important because $\mathrm{NuTeV}$ energy scale situates in the range of low to moderate $Q^{2}$, where there are few precision experiments to test the prediction powers of pQCD. This is the region where $\alpha_{S}$ is changing most rapidly, thus discrepancies could be more revealing.

The results were compared to other measurements of $\alpha_{S}$ from DIS, high energy hadron collisions, electron-positron annihilation and heavy quarkonia decay processes. The result is in better agreement with high $Q^{2}$ measurements than previous low $Q^{2}$ DIS results. Comparing the results extracted from experiments of various characteristic energy scales is an important test of pQCD.

The dominant uncertainty in the $\alpha_{S}$ measurement is due to the factorization scale dependence. The effects of the quark masses where studied and are negligible compared to the uncertainty associated with the scale dependence. Therefore, any further improvement in the determination of $\alpha_{S}$ from DIS would require improvement in theoretical calculations.

The largest experimental uncertainties are due to energy scales uncertainties. The size of these uncertainties is of the order of statistical precision. Further improvement in decrease of the experimental uncertainty could be achieved by an increased statistics (especially for non-singlet fits).

The next generation of neutrino scattering experiments, Minerva and Minos near detector, might improve the statistical precision for $\alpha_{s}$, and extend measurements to lower $Q^{2}$ scale. However, it will have the challenge of dealing with non-perturbative effects that are large at low $Q^{2}$, and energy scale uncertainties. 


\section{APPENDIX A}

\section{THE NUTEV COLLABORATION}

T. Adams ${ }^{4}$, A. Alton ${ }^{4}$, S. Avvakumov ${ }^{8}$, L. de Barbaro ${ }^{5}$, P. de Barbaro ${ }^{8}$, R. H. Bernstein ${ }^{3}$,

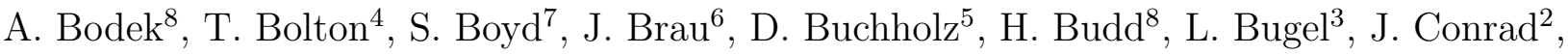
R. B. Drucker ${ }^{6}$, B. T. Fleming ${ }^{2}$, R. Frey ${ }^{6}$, J.A. Formaggio ${ }^{2}$, OlOrJ. Goldman ${ }^{4}$, M. Goncharov ${ }^{4}$, D. A. Harris ${ }^{8}$, R. A. Johnson ${ }^{1}$, J . H. Kim², S. Koutsoliotas²,

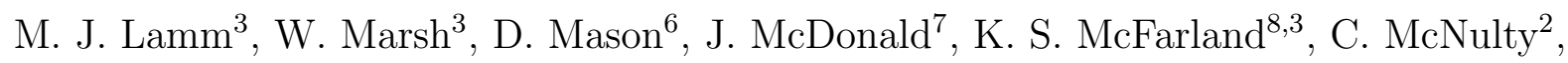
D. Naples ${ }^{7}$, P. Nienaber ${ }^{3}$, V. Radescu ${ }^{7}$, A. Romosan ${ }^{2}$, W. K. Sakumoto ${ }^{8}$, H. Schellman ${ }^{5}$, M. H. Shaevitz ${ }^{2}$, P. Spentzouris ${ }^{2}$, E. G. Stern ${ }^{2}$, N. Suwonjandee ${ }^{1}$, M. Tzanov ${ }^{7}$, M. Vakili ${ }^{1}$, A. Vaitaitis ${ }^{2}$, U. K. Yang ${ }^{8}$, J. Yu ${ }^{3}$, G. P. Zeller ${ }^{5}$ and E. D. Zimmerman ${ }^{2}$

${ }^{1}$ University of Cincinnati, Cincinnati, OH 45221

${ }^{2}$ Columbia University, New York, NY 10027

${ }^{3}$ Fermi National Accelerator Lab., Batavia, IL 60510

${ }^{4}$ Kansas State University, Manhattan, KS 66506

${ }^{5}$ Northwestern University, Evanston, IL 60208

${ }^{6}$ University of Oregon, Eugene, OR 97403

${ }^{7}$ University of Pittsburgh, Pittsburgh, PA 15260

${ }^{8}$ University of Rochester, Rochester, NY 14627 


\section{APPENDIX B}

\section{STRUCTURE FUNCTIONS TABLES}

\section{B.1 DATA TABLE FOR NON-SINGLET FIT}

Table B1 displays the $x F_{3}$ data file used in the non-singlet fit taking into account all the correlations. This table was also used for constructing the structure function covariance matrix as described in Chapter 3.

Table B1: $x F_{3}$ structure function table from 1-parameter fit. Each column corresponds to systematic uncertainties. This table was used for non-singlet fit.

\begin{tabular}{rrrrrrrrrrr}
\hline $\mathrm{x}$ & $Q^{2}$ & $x F_{3}$ & $\mathrm{stat}$ & $E_{\mu} \mathrm{scl}$ & $E_{h} \mathrm{scl}$ & $m_{c}$ & $\frac{B}{A}$ & $E_{\mu} \mathrm{sm}$ & $E_{h a d}$ sm & $\frac{B}{A}$ model \\
\hline 0.0150 & 1.2589 & 0.1799 & 0.0146 & -0.0039 & 0.0006 & -0.0003 & -0.0001 & 0.0002 & 0.0020 & 0.0032 \\
0.0150 & 1.9952 & 0.2182 & 0.0157 & -0.0077 & -0.0005 & 0.0016 & -0.0002 & 0.0038 & 0.0019 & 0.0002 \\
0.0150 & 3.1622 & 0.2634 & 0.0177 & -0.0029 & 0.0005 & -0.0016 & -0.0005 & 0.0008 & -0.0010 & 0.0014 \\
0.0150 & 5.0118 & 0.3262 & 0.0212 & -0.0069 & -0.0006 & 0.0028 & -0.0008 & 0.0142 & 0.0017 & 0.0129 \\
0.0150 & 7.9432 & 0.3026 & 0.0621 & -0.0288 & 0.0127 & -0.0001 & -0.0010 & 0.0097 & 0.0000 & 0.0065 \\
0.0450 & 1.2589 & 0.3755 & 0.0346 & 0.0000 & -0.0013 & -0.0075 & -0.0011 & 0.0107 & 0.0103 & 0.0038 \\
0.0450 & 1.9952 & 0.4007 & 0.0211 & -0.0167 & -0.0021 & -0.0022 & -0.0002 & 0.0114 & 0.0001 & 0.0078 \\
0.0450 & 3.1622 & 0.4655 & 0.0176 & 0.0001 & -0.0030 & -0.0033 & -0.0001 & 0.0024 & 0.0053 & 0.0044 \\
0.0450 & 5.0118 & 0.4829 & 0.0160 & -0.0109 & 0.0017 & -0.0009 & -0.0005 & 0.0033 & -0.0014 & 0.0082 \\
0.0450 & 7.9432 & 0.4908 & 0.0185 & -0.0097 & -0.0006 & 0.0008 & -0.0010 & 0.0042 & 0.0012 & 0.0034 \\
0.0450 & 12.5890 & 0.5400 & 0.0197 & -0.0076 & -0.0012 & 0.0037 & -0.0015 & 0.0023 & -0.0004 & 0.0030 \\
0.0450 & 19.9520 & 0.5729 & 0.0429 & -0.0361 & 0.0284 & 0.0186 & -0.0018 & 0.0070 & 0.0137 & 0.0171 \\
0.0800 & 1.9952 & 0.5362 & 0.0337 & -0.0116 & -0.0111 & -0.0010 & -0.0010 & 0.0035 & 0.0060 & 0.0014 \\
0.0800 & 3.1622 & 0.6031 & 0.0229 & -0.0061 & -0.0109 & -0.0094 & 0.0000 & 0.0249 & 0.0017 & 0.0035 \\
0.0800 & 5.0118 & 0.6596 & 0.0173 & -0.0058 & 0.0013 & -0.0032 & 0.0000 & 0.0083 & 0.0006 & 0.0086 \\
0.0800 & 7.9432 & 0.6441 & 0.0142 & -0.0117 & -0.0044 & -0.0040 & -0.0005 & 0.0104 & 0.0028 & 0.0079 \\
0.0800 & 12.5890 & 0.6651 & 0.0165 & -0.0059 & 0.0059 & -0.0003 & -0.0013 & 0.0092 & -0.0051 & 0.0027 \\
0.0800 & 19.9520 & 0.6790 & 0.0164 & -0.0086 & 0.0026 & -0.0004 & -0.0019 & 0.0162 & 0.0014 & 0.0100 \\
\hline
\end{tabular}




\begin{tabular}{|c|c|c|c|c|c|c|c|c|c|c|}
\hline \multicolumn{11}{|c|}{ Continued } \\
\hline $\mathrm{x}$ & $Q^{2}$ & $x F_{3}$ & stat & $E_{\mu} \mathrm{scl}$ & $E_{h} \mathrm{scl}$ & $m_{c}$ & $\frac{B}{A}$ & $E_{\mu} \mathrm{sm}$ & $E_{\text {had }} \mathrm{sm}$ & $\frac{B}{A}$ model \\
\hline 0.0800 & 31.6220 & 0.7531 & 0.0266 & -0.0235 & 0.0141 & 0.0086 & -0.0024 & 0.0148 & -0.0010 & 0.0116 \\
\hline 0.1250 & 3.1622 & 0.6410 & 0.0321 & -0.0021 & -0.0041 & -0.0132 & -0.0010 & 0.0001 & 0.0068 & 0.0103 \\
\hline 0.1250 & 5.0118 & 0.6980 & 0.0213 & -0.0103 & 0.0023 & -0.0161 & 0.0001 & 0.0046 & 0.0045 & 0.0015 \\
\hline 0.1250 & 7.9432 & 0.7373 & 0.0145 & -0.0064 & 0.0026 & 0.0022 & 0.0001 & 0.0036 & 0.0011 & 0.0093 \\
\hline 0.1250 & 12.5890 & 0.7687 & 0.0135 & -0.0136 & 0.0016 & -0.0018 & -0.0008 & 0.0053 & 0.0021 & 0.0032 \\
\hline 0.1250 & 19.9520 & 0.7677 & 0.0148 & -0.0137 & 0.0066 & 0.0014 & -0.0016 & 0.0097 & 0.0031 & 0.0012 \\
\hline 0.1250 & 31.6220 & 0.7634 & 0.0143 & -0.0093 & 0.0024 & 0.0056 & -0.0022 & 0.0021 & 0.0010 & 0.0037 \\
\hline 0.1250 & 50.1180 & 0.8024 & 0.0256 & -0.0082 & 0.0204 & 0.0144 & -0.0026 & 0.0019 & 0.0017 & 0.0067 \\
\hline 0.1750 & 3.1622 & 0.6167 & 0.0857 & -0.0021 & 0.0007 & -0.0269 & -0.0010 & 0.0115 & 0.0396 & 0.0149 \\
\hline 0.1750 & 5.0118 & 0.7514 & 0.0314 & -0.0008 & -0.0068 & -0.0152 & -0.0001 & 0.0036 & -0.0021 & 0.0007 \\
\hline 0.1750 & 7.9432 & 0.7710 & 0.0170 & -0.0054 & 0.0043 & -0.0009 & 0.0003 & 0.0005 & 0.0043 & 0.0019 \\
\hline 0.1750 & 12.5890 & 0.8087 & 0.0156 & -0.0051 & 0.0010 & -0.0009 & -0.0004 & 0.0039 & 0.0015 & 0.0015 \\
\hline 0.1750 & 19.9520 & 0.7962 & 0.0129 & -0.0079 & 0.0034 & 0.0003 & -0.0009 & 0.0070 & -0.0035 & 0.0024 \\
\hline 0.1750 & 31.6220 & 0.7608 & 0.0145 & -0.0087 & -0.0017 & 0.0006 & -0.0019 & 0.0034 & -0.0040 & 0.0037 \\
\hline 0.1750 & 50.1180 & 0.7853 & 0.0139 & -0.0149 & 0.0040 & 0.0017 & -0.0024 & 0.0027 & 0.0016 & 0.0001 \\
\hline 0.2250 & 5.0118 & 0.7818 & 0.0467 & -0.0126 & 0.0034 & -0.0265 & -0.0005 & 0.0282 & 0.0073 & 0.0034 \\
\hline 0.2250 & 7.9432 & 0.7667 & 0.0239 & 0.0032 & -0.0029 & -0.0047 & 0.0003 & 0.0038 & -0.0011 & 0.0012 \\
\hline 0.2250 & 12.5890 & 0.7600 & 0.0156 & 0.0039 & -0.0046 & -0.0023 & 0.0002 & 0.0015 & 0.0055 & 0.0001 \\
\hline 0.2250 & 19.9520 & 0.7871 & 0.0136 & -0.0004 & 0.0034 & -0.0030 & -0.0007 & 0.0164 & -0.0016 & 0.0120 \\
\hline 0.2250 & 31.6220 & 0.7413 & 0.0137 & -0.0039 & 0.0057 & 0.0003 & -0.0015 & 0.0032 & 0.0010 & 0.0002 \\
\hline 0.2250 & 50.1180 & 0.7149 & 0.0131 & -0.0085 & 0.0008 & 0.0013 & -0.0020 & 0.0039 & -0.0009 & 0.0066 \\
\hline 0.2250 & 79.4320 & 0.6968 & 0.0178 & -0.0095 & 0.0100 & 0.0100 & -0.0023 & 0.0073 & 0.0026 & 0.0075 \\
\hline 0.2750 & 5.0118 & 0.6842 & 0.0829 & 0.0124 & -0.0130 & -0.0106 & -0.0006 & 0.0770 & -0.0009 & 0.0742 \\
\hline 0.2750 & 7.9432 & 0.7264 & 0.0261 & -0.0053 & 0.0008 & -0.0056 & 0.0003 & 0.0014 & 0.0056 & 0.0129 \\
\hline 0.2750 & 12.5890 & 0.7057 & 0.0183 & 0.0051 & -0.0066 & -0.0003 & 0.0001 & 0.0010 & 0.0020 & 0.0058 \\
\hline 0.2750 & 19.9520 & 0.6791 & 0.0151 & -0.0074 & 0.0040 & -0.0008 & -0.0003 & 0.0020 & 0.0020 & 0.0001 \\
\hline 0.2750 & 31.6220 & 0.6812 & 0.0132 & 0.0021 & 0.0036 & 0.0035 & -0.0011 & 0.0097 & 0.0006 & 0.0038 \\
\hline 0.2750 & 50.1180 & 0.6387 & 0.0141 & 0.0037 & -0.0030 & 0.0008 & -0.0017 & 0.0037 & 0.0014 & 0.0015 \\
\hline 0.2750 & 79.4320 & 0.6480 & 0.0142 & -0.0160 & 0.0066 & 0.0037 & -0.0021 & 0.0078 & -0.0008 & 0.0027 \\
\hline 0.3500 & 7.9432 & 0.5658 & 0.0294 & 0.0120 & -0.0026 & -0.0047 & -0.0002 & 0.0040 & 0.0025 & 0.0052 \\
\hline 0.3500 & 12.5890 & 0.5789 & 0.0154 & 0.0080 & 0.0011 & -0.0005 & 0.0002 & 0.0017 & 0.0008 & 0.0060 \\
\hline 0.3500 & 19.9520 & 0.5721 & 0.0112 & -0.0002 & -0.0002 & -0.0045 & 0.0000 & 0.0074 & 0.0016 & 0.0007 \\
\hline 0.3500 & 31.6220 & 0.5355 & 0.0087 & 0.0012 & -0.0022 & 0.0003 & -0.0006 & 0.0012 & 0.0003 & 0.0003 \\
\hline 0.3500 & 50.1180 & 0.5144 & 0.0100 & -0.0001 & 0.0007 & -0.0021 & -0.0012 & 0.0044 & -0.0010 & 0.0001 \\
\hline 0.3500 & 79.4320 & 0.5018 & 0.0082 & -0.0003 & 0.0003 & 0.0023 & -0.0015 & 0.0009 & 0.0017 & 0.0000 \\
\hline 0.4500 & 7.9432 & 0.4050 & 0.0446 & 0.0190 & -0.0153 & -0.0176 & -0.0003 & 0.0236 & 0.0000 & 0.0360 \\
\hline 0.4500 & 12.5890 & 0.3878 & 0.0169 & 0.0142 & -0.0106 & -0.0009 & 0.0002 & 0.0053 & -0.0008 & 0.0014 \\
\hline 0.4500 & 19.9520 & 0.3787 & 0.0113 & 0.0120 & -0.0032 & 0.0006 & 0.0000 & 0.0015 & 0.0009 & 0.0047 \\
\hline 0.4500 & 31.6220 & 0.3489 & 0.0086 & 0.0088 & -0.0039 & -0.0011 & -0.0003 & 0.0038 & 0.0016 & 0.0036 \\
\hline 0.4500 & 50.1180 & 0.3187 & 0.0086 & 0.0117 & -0.0023 & 0.0010 & -0.0006 & 0.0009 & -0.0002 & 0.0005 \\
\hline 0.4500 & 79.4320 & 0.2965 & 0.0075 & 0.0019 & -0.0026 & -0.0008 & -0.0009 & 0.0016 & 0.0008 & 0.0013 \\
\hline 0.4500 & 125.8900 & 0.2913 & 0.0093 & 0.0089 & 0.0067 & 0.0008 & -0.0009 & 0.0022 & -0.0011 & 0.0031 \\
\hline 0.5500 & 12.5890 & 0.2435 & 0.0192 & -0.0012 & -0.0046 & -0.0047 & -0.0001 & 0.0029 & -0.0005 & 0.0054 \\
\hline 0.5500 & 19.9520 & 0.2219 & 0.0105 & 0.0074 & 0.0015 & -0.0021 & 0.0000 & 0.0028 & -0.0001 & 0.0024 \\
\hline 0.5500 & 31.6220 & 0.2097 & 0.0077 & -0.0003 & -0.0021 & 0.0009 & -0.0002 & 0.0031 & 0.0010 & 0.0029 \\
\hline 0.5500 & 50.1180 & 0.1801 & 0.0068 & 0.0078 & -0.0011 & -0.0001 & -0.0003 & 0.0017 & -0.0017 & 0.0001 \\
\hline 0.5500 & 79.4320 & 0.1635 & 0.0065 & 0.0010 & 0.0015 & -0.0026 & -0.0004 & 0.0029 & -0.0002 & 0.0037 \\
\hline 0.5500 & 125.8900 & 0.1341 & 0.0085 & 0.0054 & 0.0001 & 0.0021 & -0.0005 & 0.0043 & 0.0017 & 0.0032 \\
\hline 0.6500 & 12.5890 & 0.1250 & 0.0177 & 0.0079 & 0.0047 & 0.0037 & 0.0002 & 0.0055 & 0.0048 & 0.0003 \\
\hline 0.6500 & 19.9520 & 0.1252 & 0.0091 & 0.0065 & -0.0047 & 0.0003 & 0.0001 & 0.0001 & 0.0016 & 0.0031 \\
\hline 0.6500 & 31.6220 & 0.1158 & 0.0061 & 0.0058 & -0.0013 & 0.0004 & 0.0000 & 0.0018 & -0.0012 & 0.0013 \\
\hline 0.6500 & 50.1180 & 0.0844 & 0.0055 & 0.0041 & -0.0005 & -0.0005 & -0.0001 & 0.0014 & 0.0015 & 0.0001 \\
\hline 0.6500 & 79.4320 & 0.0713 & 0.0060 & 0.0016 & -0.0010 & 0.0015 & -0.0002 & 0.0006 & 0.0013 & 0.0004 \\
\hline 0.6500 & 125.8900 & 0.0702 & 0.0063 & 0.0058 & 0.0009 & -0.0016 & -0.0002 & 0.0017 & 0.0017 & 0.0037 \\
\hline 0.7500 & 19.9520 & 0.0501 & 0.0070 & 0.0024 & 0.0006 & -0.0023 & 0.0000 & 0.0002 & 0.0004 & 0.0011 \\
\hline 0.7500 & 31.6220 & 0.0379 & 0.0040 & 0.0044 & -0.0019 & -0.0005 & 0.0000 & 0.0005 & -0.0006 & 0.0011 \\
\hline 0.7500 & 50.1180 & 0.0338 & 0.0042 & 0.0022 & 0.0012 & -0.0016 & -0.0001 & 0.0004 & 0.0003 & 0.0009 \\
\hline
\end{tabular}




\section{B.2 DATA TABLE FOR COMBINED FIT}

Table B2 displays the correlated $F_{2}$ and $x F_{3}$ data file used in the combined fit taking into account all the correlations.

Table B2: $F_{2}$ and $x F_{3}$ structure function from simultaneous fit to differential cross-section using the covariance matrix taking into account point-to-point correlations.

\begin{tabular}{|c|c|c|c|c|c|c|c|c|c|c|c|c|}
\hline $\mathrm{x}$ & $Q^{2}$ & $\mathrm{~F} 2$ & F2err & F2Rsys & F2msys & $\mathrm{xF} 3$ & XF3err & xF3Rsys & XF3msys & F2xF3corr & xbin & Q2bin \\
\hline 0.015 & 1.259 & 1.1550000 & 0.0249800 & -0.0020000 & 0.0090000 & 0.1821000 & 0.0389500 & 0.0022000 & -0.0002500 & -0.1148000 & 2 & 4 \\
\hline 0.015 & 1.995 & 1.3300000 & 0.0307700 & 0.0029999 & 0.0130000 & 0.2170000 & 0.0417500 & 0.0013000 & -0.0007500 & -0.1582000 & 2 & 5 \\
\hline 0.015 & 3.162 & 1.4730000 & 0.0397300 & -0.0010000 & 0.0180000 & 0.2669000 & 0.0470000 & 0.0010000 & -0.0005500 & -0.2252000 & 2 & 6 \\
\hline 0.015 & 5.012 & 1.5580000 & 0.0591100 & 0.0080000 & 0.0270000 & 0.3220000 & 0.0575200 & 0.0006000 & -0.0002000 & -0.2393000 & 2 & 7 \\
\hline 0.015 & 7.943 & 1.6150000 & 0.1822000 & 0.0240000 & 0.0320000 & 0.2751000 & 0.1626000 & 0.0005000 & 0.0005000 & -0.3664000 & 2 & 8 \\
\hline 0.045 & 1.259 & 1.0930001 & 0.0246100 & -0.0030000 & 0.0015000 & 0.3758000 & 0.0866300 & 0.0045000 & -0.0002000 & -0.0426200 & 3 & 4 \\
\hline 0.045 & 1.995 & 1.2280000 & 0.0232800 & 0.0000000 & 0.0040000 & 0.4073000 & 0.0526400 & 0.0025000 & -0.0008000 & 0.0078580 & 3 & 5 \\
\hline 0.045 & 3.162 & 1.3430001 & 0.0243200 & 0.0050000 & 0.0060000 & 0.4627000 & 0.0443200 & 0.0013000 & -0.0007500 & -0.0010020 & 3 & 6 \\
\hline 0.045 & 5.012 & 1.4590000 & 0.0274700 & 0.0120000 & 0.0095000 & 0.4747000 & 0.0398900 & -0.0001000 & -0.0016500 & 0.0096090 & 3 & 7 \\
\hline 0.045 & 7.943 & 1.5120000 & 0.0333000 & 0.0180000 & 0.0105000 & 0.5004000 & 0.0454700 & -0.0004000 & -0.0015000 & -0.0933900 & 3 & 8 \\
\hline 0.045 & 12.589 & 1.5510000 & 0.0446700 & 0.0270000 & 0.0160000 & 0.5374000 & 0.0491000 & 0.0000000 & -0.0005000 & -0.1220000 & 3 & 9 \\
\hline 0.045 & 19.952 & 1.6840000 & 0.1085000 & 0.0290000 & 0.0215001 & 0.5691000 & 0.1049000 & 0.0001000 & -0.0001500 & -0.1893000 & 3 & 10 \\
\hline 0.080 & 1.259 & 1.0670000 & 0.0670500 & -0.0020000 & 0.0000000 & 0.4083000 & 0.4714000 & -0.0075000 & 0.0000000 & -0.1067000 & 4 & 4 \\
\hline 0.080 & 1.995 & 1.2180001 & 0.0222000 & -0.0010000 & 0.0015000 & 0.5397000 & 0.0810800 & -0.0003000 & -0.0003500 & 0.0002854 & 4 & 5 \\
\hline 0.080 & 3.162 & 1.3020000 & 0.0220500 & 0.0009999 & 0.0025000 & 0.6069000 & 0.0550300 & 0.0001000 & -0.0007500 & 0.0525000 & 4 & 6 \\
\hline 0.080 & 5.012 & 1.3600000 & 0.0210800 & 0.0070000 & 0.0045000 & 0.6616000 & 0.0411400 & -0.0005000 & -0.0012500 & 0.0835200 & 4 & 7 \\
\hline 0.080 & 7.943 & 1.3770000 & 0.0217100 & 0.0110000 & 0.0065000 & 0.6383000 & 0.0337400 & -0.0011000 & -0.0015500 & 0.1116000 & 4 & 8 \\
\hline 0.080 & 12.589 & 1.4230000 & 0.0273800 & 0.0160000 & 0.0070000 & 0.6608000 & 0.0387700 & -0.0012000 & -0.0015500 & 0.0468200 & 4 & 9 \\
\hline 0.080 & 19.952 & 1.4220001 & 0.0325600 & 0.0189999 & 0.0100001 & 0.6794000 & 0.0387400 & -0.0006000 & -0.0007500 & 0.0158000 & 4 & 10 \\
\hline 0.080 & 31.622 & 1.4930000 & 0.0613700 & 0.0210000 & 0.0130000 & 0.7281000 & 0.0630800 & -0.0001000 & -0.0003000 & -0.0001793 & 4 & 11 \\
\hline 0.125 & 1.995 & 1.1350000 & 0.0631500 & -0.0010000 & 0.0000000 & 0.7274000 & 0.4465000 & -0.0185000 & -0.0000500 & -0.0676600 & 5 & 5 \\
\hline 0.125 & 3.162 & 1.2340000 & 0.0197700 & -0.0009999 & 0.0010000 & 0.6374000 & 0.0740200 & -0.0046999 & -0.0005000 & 0.0164800 & 5 & 6 \\
\hline 0.125 & 5.012 & 1.2490000 & 0.0192600 & 0.0020001 & 0.0020000 & 0.6928000 & 0.0492200 & -0.0022000 & -0.0009000 & 0.1099000 & 5 & 7 \\
\hline 0.125 & 7.943 & 1.2560000 & 0.0171300 & 0.0050000 & 0.0030000 & 0.7372000 & 0.0331900 & -0.0019000 & -0.0013000 & 0.1605000 & 5 & 8 \\
\hline 0.125 & 12.589 & 1.2819999 & 0.0195700 & 0.0080000 & 0.0040000 & 0.7616000 & 0.0309000 & -0.0018000 & -0.0015500 & 0.2111000 & 5 & 9 \\
\hline 0.125 & 19.952 & 1.2680000 & 0.0232600 & 0.0100000 & 0.0045000 & 0.7594000 & 0.0335400 & -0.0014000 & -0.0012000 & 0.1628000 & 5 & 10 \\
\hline 0.125 & 31.622 & 1.2530000 & 0.0267100 & 0.0130000 & 0.0060000 & 0.7665000 & 0.0321900 & -0.0006000 & -0.0006000 & 0.1593000 & 5 & 11 \\
\hline 0.125 & 50.118 & 1.3230000 & 0.0553400 & 0.0130000 & 0.0075000 & 0.7888000 & 0.0586500 & -0.0002000 & -0.0002000 & 0.1438000 & 5 & 12 \\
\hline 0.175 & 3.162 & 1.1250000 & 0.0366500 & 0.0000000 & 0.0005000 & 0.5863000 & 0.1922000 & 0.0000000 & -0.0001500 & -0.0296100 & 6 & 6 \\
\hline 0.175 & 5.012 & 1.1210001 & 0.0192700 & 0.0000000 & 0.0005000 & 0.7508000 & 0.0702800 & 0.0000000 & -0.0004500 & 0.0716300 & 6 & 7 \\
\hline 0.175 & 7.943 & 1.1580000 & 0.0163600 & 0.0000000 & 0.0015000 & 0.7746000 & 0.0376400 & 0.0000000 & -0.0009500 & 0.1854000 & 6 & 8 \\
\hline 0.175 & 12.589 & 1.1540000 & 0.0174800 & 0.0000000 & 0.0020000 & 0.7929000 & 0.0339100 & 0.0000000 & -0.0011000 & 0.2218000 & 6 & 9 \\
\hline 0.175 & 19.952 & 1.1390001 & 0.0183100 & 0.0000000 & 0.0030000 & 0.7779000 & 0.0280100 & 0.0000000 & -0.0011000 & 0.2842000 & 6 & 10 \\
\hline 0.175 & 31.622 & 1.1310000 & 0.0224600 & 0.0000000 & 0.0030000 & 0.7667000 & 0.0314500 & 0.0000000 & -0.0008500 & 0.2304000 & 6 & 11 \\
\hline 0.175 & 50.118 & 1.0940000 & 0.0266200 & 0.0000000 & 0.0045000 & 0.7843000 & 0.0308400 & 0.0000000 & -0.0003500 & 0.3463000 & 6 & 12 \\
\hline 0.175 & 79.432 & 1.1520000 & 0.0830000 & 0.0000000 & 0.0050000 & 0.8030000 & 0.0861200 & 0.0000000 & 0.0000000 & 0.3541000 & 6 & 13 \\
\hline 0.225 & 5.012 & 1.0330000 & 0.0221300 & 0.0000000 & 0.0000000 & 0.7713000 & 0.1014000 & 0.0000000 & -0.0003000 & 0.0535300 & 7 & 7 \\
\hline 0.225 & 7.943 & 1.0260000 & 0.0173400 & 0.0000000 & 0.0010000 & 0.7582000 & 0.0523300 & 0.0000000 & -0.0004500 & 0.1415000 & 7 & 8 \\
\hline 0.225 & 12.589 & 1.0000000 & 0.0154600 & 0.0000000 & 0.0010000 & 0.7483000 & 0.0334600 & 0.0000000 & -0.0008500 & 0.2449000 & 7 & 9 \\
\hline 0.225 & 19.952 & 0.9956000 & 0.0170600 & 0.0000000 & 0.0016000 & 0.7646000 & 0.0288100 & 0.0000000 & -0.0009000 & 0.3485000 & 7 & 10 \\
\hline 0.225 & 31.622 & 0.9545000 & 0.0185100 & 0.0000000 & 0.0016500 & 0.7380000 & 0.0286700 & 0.0000000 & -0.0007500 & 0.3163000 & 7 & 11 \\
\hline 0.225 & 50.118 & 0.9246000 & 0.0211400 & 0.0000000 & 0.0020500 & 0.7118000 & 0.0278000 & 0.0000000 & -0.0004500 & 0.3358000 & 7 & 12 \\
\hline 0.225 & 79.432 & 0.9121000 & 0.0345700 & 0.0000000 & 0.0027500 & 0.6938000 & 0.0385200 & 0.0000000 & -0.0001500 & 0.4089000 & 7 & 13 \\
\hline
\end{tabular}




\begin{tabular}{|c|c|c|c|c|c|c|c|c|c|c|c|c|}
\hline \multicolumn{13}{|c|}{ Continued } \\
\hline $\mathrm{x}$ & $Q^{2}$ & F2 & F2err & F2Rsys & F2msys & $\mathrm{xF} 3$ & XF3err & xF3Rsys & XF3msys & F2xF3corr & xbin & Q2bin \\
\hline 0.275 & 5.012 & 0.9046000 & 0.0331400 & 0.0000000 & 0.0002500 & 0.6478000 & 0.1758000 & 0.0000000 & -0.0001500 & 0.0266200 & 8 & 7 \\
\hline 0.275 & 7.943 & 0.8831000 & 0.0163400 & 0.0000000 & 0.0005500 & 0.7323000 & 0.0555700 & 0.0000000 & -0.0004500 & 0.1529000 & 8 & 8 \\
\hline 0.275 & 12.589 & 0.8751000 & 0.0155300 & 0.0000000 & 0.0007500 & 0.6952000 & 0.0387100 & 0.0000000 & -0.0005000 & 0.2369000 & 8 & 9 \\
\hline 0.275 & 19.952 & 0.8424000 & 0.0154300 & 0.0000000 & 0.0008000 & 0.6615000 & 0.0315400 & 0.0000000 & -0.0005500 & 0.2618000 & 8 & 10 \\
\hline 0.275 & 31.622 & 0.8244000 & 0.0162400 & 0.0000000 & 0.0010500 & 0.6654000 & 0.0269100 & 0.0000000 & -0.0006000 & 0.3419000 & 8 & 11 \\
\hline 0.275 & 50.118 & 0.7680000 & 0.0198900 & 0.0000000 & 0.0011500 & 0.6425000 & 0.0285100 & 0.0000000 & -0.0003500 & 0.3719000 & 8 & 12 \\
\hline 0.275 & 79.432 & 0.7539000 & 0.0249300 & 0.0000000 & 0.0015000 & 0.6400000 & 0.0303300 & 0.0000000 & -0.0001000 & 0.4588000 & 8 & 13 \\
\hline 0.350 & 7.943 & 0.6850000 & 0.0130700 & 0.0000000 & 0.0002000 & 0.5490000 & 0.0610600 & 0.0000000 & -0.0002000 & 0.0825400 & 9 & 8 \\
\hline 0.350 & 12.589 & 0.6721000 & 0.0099800 & 0.0000000 & 0.0003000 & 0.5713000 & 0.0320600 & 0.0000000 & -0.0002000 & 0.1804000 & 9 & 9 \\
\hline 0.350 & 19.952 & 0.6320000 & 0.0095910 & 0.0000000 & 0.0004000 & 0.5561000 & 0.0228700 & 0.0000000 & -0.0003000 & 0.2740000 & 9 & 10 \\
\hline 0.350 & 31.622 & 0.5922000 & 0.0095350 & 0.0000000 & 0.0005500 & 0.5332000 & 0.0172400 & 0.0000000 & -0.0003000 & 0.3948000 & 9 & 11 \\
\hline 0.350 & 50.118 & 0.5630000 & 0.0116100 & 0.0000000 & 0.0005500 & 0.5214000 & 0.0190000 & 0.0000000 & -0.0003000 & 0.4014000 & 9 & 12 \\
\hline 0.350 & 79.432 & 0.5415000 & 0.0118600 & 0.0000000 & 0.0006000 & 0.5037000 & 0.0161600 & 0.0000000 & -0.0002000 & 0.4878000 & 9 & 13 \\
\hline 0.350 & 125.890 & 0.5202000 & 0.0257600 & 0.0000000 & 0.0008500 & 0.4785000 & 0.0301400 & 0.0000000 & -0.0000500 & 0.6140000 & 9 & 14 \\
\hline 0.450 & 7.943 & 0.4467000 & 0.0168000 & 0.0000000 & 0.0001000 & 0.3817000 & 0.0896800 & 0.0000000 & -0.0001000 & 0.0536800 & 10 & 8 \\
\hline 0.450 & 12.589 & 0.4236000 & 0.0086880 & 0.0000000 & 0.0001000 & 0.3838000 & 0.0347700 & 0.0000000 & -0.0001000 & 0.1406000 & 10 & 9 \\
\hline 0.450 & 19.952 & 0.3950000 & 0.0079170 & 0.0000000 & 0.0001500 & 0.3735000 & 0.0230200 & 0.0000000 & -0.0001000 & 0.2461000 & 10 & 10 \\
\hline 0.450 & 31.622 & 0.3615000 & 0.0075300 & 0.0000000 & 0.0001500 & 0.3425000 & 0.0170500 & 0.0000000 & -0.0001000 & 0.3342000 & 10 & 11 \\
\hline 0.450 & 50.118 & 0.3417000 & 0.0085740 & 0.0000000 & 0.0002000 & 0.3222000 & 0.0167200 & 0.0000000 & -0.0001000 & 0.3376000 & 10 & 12 \\
\hline 0.450 & 79.432 & 0.3130000 & 0.0085700 & 0.0000000 & 0.0002000 & 0.2990000 & 0.0143000 & 0.0000000 & -0.0000500 & 0.3491000 & 10 & 13 \\
\hline 0.450 & 125.890 & 0.3038000 & 0.0135800 & 0.0000000 & 0.0003000 & 0.2884000 & 0.0180400 & 0.0000000 & -0.0000500 & 0.4808000 & 10 & 14 \\
\hline 0.550 & 12.589 & 0.2548000 & 0.0077040 & 0.0000000 & 0.0000500 & 0.2347000 & 0.0382700 & 0.0000000 & -0.0000500 & 0.0767800 & 11 & 9 \\
\hline 0.550 & 19.952 & 0.2248000 & 0.0056970 & 0.0000000 & 0.0000500 & 0.2121000 & 0.0209700 & 0.0000000 & -0.0000500 & 0.1793000 & 11 & 10 \\
\hline 0.550 & 31.622 & 0.2075000 & 0.0054880 & 0.0000000 & 0.0000500 & 0.2044000 & 0.0154400 & 0.0000000 & -0.0000500 & 0.2163000 & 11 & 11 \\
\hline 0.550 & 50.118 & 0.1772000 & 0.0061200 & 0.0000000 & 0.0000500 & 0.1843000 & 0.0128400 & 0.0000000 & -0.0000500 & 0.3785000 & 11 & 12 \\
\hline 0.550 & 79.432 & 0.1607000 & 0.0061050 & 0.0000000 & 0.0001000 & 0.1622000 & 0.0124600 & 0.0000000 & -0.0000500 & 0.2576000 & 11 & 13 \\
\hline 0.550 & 125.890 & 0.1569000 & 0.0103700 & 0.0000000 & 0.0001000 & 0.1347000 & 0.0159400 & 0.0000000 & 0.0000000 & 0.2513000 & 11 & 14 \\
\hline 0.650 & 12.589 & 0.1362000 & 0.0066600 & 0.0000000 & 0.0000500 & 0.1330000 & 0.0339500 & 0.0000000 & -0.0000500 & 0.0966300 & 12 & 9 \\
\hline 0.650 & 19.952 & 0.1150000 & 0.0042000 & 0.0000000 & 0.0000500 & 0.1206000 & 0.0177200 & 0.0000000 & 0.0000000 & 0.1804000 & 12 & 10 \\
\hline 0.650 & 31.622 & 0.0985200 & 0.0035160 & 0.0000000 & 0.0000200 & 0.1126000 & 0.0116800 & 0.0000000 & -0.0000500 & 0.2456000 & 12 & 11 \\
\hline 0.650 & 50.118 & 0.0875500 & 0.0039140 & 0.0000000 & 0.0000250 & 0.0832300 & 0.0103100 & 0.0000000 & -0.0000150 & 0.2288000 & 12 & 12 \\
\hline 0.650 & 79.432 & 0.0785800 & 0.0043430 & 0.0000000 & 0.0000200 & 0.0718600 & 0.0107200 & 0.0000000 & -0.0000100 & 0.1101000 & 12 & 13 \\
\hline 0.650 & 125.890 & 0.0707100 & 0.0061910 & 0.0000000 & 0.0000250 & 0.0696300 & 0.0117300 & 0.0000000 & 0.0000050 & 0.2309000 & 12 & 14 \\
\hline 0.750 & 12.589 & 0.0626300 & 0.0051450 & 0.0000000 & 0.0000150 & 0.0499400 & 0.0292900 & 0.0000000 & -0.0000050 & 0.0304500 & 13 & 9 \\
\hline 0.750 & 19.952 & 0.0495000 & 0.0026160 & 0.0000000 & 0.0000050 & 0.0479300 & 0.0134100 & 0.0000000 & -0.0000050 & 0.1028000 & 13 & 10 \\
\hline 0.750 & 31.622 & 0.0381800 & 0.0020210 & 0.0000000 & 0.0000050 & 0.0351600 & 0.0075340 & 0.0000000 & 0.0000000 & 0.0904300 & 13 & 11 \\
\hline 0.750 & 50.118 & 0.0332900 & 0.0020300 & 0.0000000 & 0.0000050 & 0.0343500 & 0.0077230 & 0.0000000 & -0.0000050 & 0.0541000 & 13 & 12 \\
\hline 0.750 & 79.432 & 0.0308300 & 0.0030310 & 0.0000000 & 0.0000050 & 0.0254000 & 0.0100100 & 0.0000000 & 0.0000050 & -0.1176000 & 13 & 13 \\
\hline
\end{tabular}




\section{APPENDIX C}

\section{QCD SOURCE CODE: PART A}

This Appendix contains relevant portions of the QCD program used in this analysis: the main program, the fitter, and the evolution programs. Table $\mathrm{C}$ summarizes the purpose of key QCD programs. The QCD program is a result of fusion of two programs:

- calculation of structure function taking into account heavy quark treatment [54];

- massless quark evolution, fitting, and calculation of structure functions in a massless $\overline{M S}$ scheme [53]. 
Table C1: Summary of the QCD program.

\begin{tabular}{|c|c|c|}
\hline Program Name & Purpose & Description \\
\hline altpfit02.f & Main program & $\begin{array}{l}\text { Reads in the input file, stores data points in an } \\
\text { array (after cuts), calls minimization, writes } \\
\text { the output. }\end{array}$ \\
\hline minim.f & Minimization program & $\begin{array}{l}\text { Marquardt algorithm; calls datscn which cal- } \\
\text { culates } \chi^{2}, \alpha, \beta \text {. It returns } \chi^{2} \text { and errors. }\end{array}$ \\
\hline altpar02.f & Param. and evol. PDFs & $\begin{array}{l}\text { Parametrizes PDFs at initial } Q_{0}^{2} \text {. Evolves } \\
\text { massless PDFs. QCD sum rules are applied } \\
\text { here. }\end{array}$ \\
\hline theory02.f & Theory calculations & $\begin{array}{l}\text { First call stores the evolved PDFs in a grid. } \\
\text { Through a flag choose } \overline{M S} \text { model (theory } 2 . \mathrm{f} \text { ) } \\
\text { or } A C O T \text { model (theory_fo.f). Target Mass } \\
\text { correction is applied here. }\end{array}$ \\
\hline alpha_cwz.f & Calculates $\alpha_{S}$ & $\begin{array}{l}\text { Given } \Lambda_{Q C D} \text { and } Q^{2} \text { it returns } n_{f} \text { and } \alpha_{S} \text {. } \\
\text { The threshold quark masses are set here. }\end{array}$ \\
\hline cc103.f & Returns Structure Functions $(A C O T)$ & $\begin{array}{l}\text { fcc123 subroutine loops over all interacting } \\
\text { quarks and returns structure functions under } \\
\text { every scheme. CKM couplings applied here. } \\
\text { The quark masses stored in the common block } \\
\text { xmarray. }\end{array}$ \\
\hline tot104.f & Adds all contributing graphs & $\begin{array}{l}\text { The schemes are defined here: sums the con- } \\
\text { tributing diagrams. }\end{array}$ \\
\hline unified104.f & Computes each graph & $\begin{array}{l}\text { Calculations are made in helicity basis and } \\
\text { then transformed to tensor basis. }\end{array}$ \\
\hline fredpdf.f & Patch PDFs & $\begin{array}{l}\text { This is where the call to the parametrized } \\
\text { PDFs is made (pdfjo is called here). }\end{array}$ \\
\hline alphas70.f & use J. Owens $\alpha_{S}$ & $\begin{array}{l}\text { Adjusted to use J.Owens } \alpha_{S} \text { calculation in al- } \\
\text { pha_cwz.f. }\end{array}$ \\
\hline utilities103.f & technical tools & $\begin{array}{l}\text { It contains tools needed to perform the calcu- } \\
\text { lations in } A C O T \text { mode(integrations). }\end{array}$ \\
\hline
\end{tabular}




\section{APPENDIX D}

QCD PROGRAM: FITTER, EVOLUTION, $\overline{M S}$ 




Figure D1: Control file 

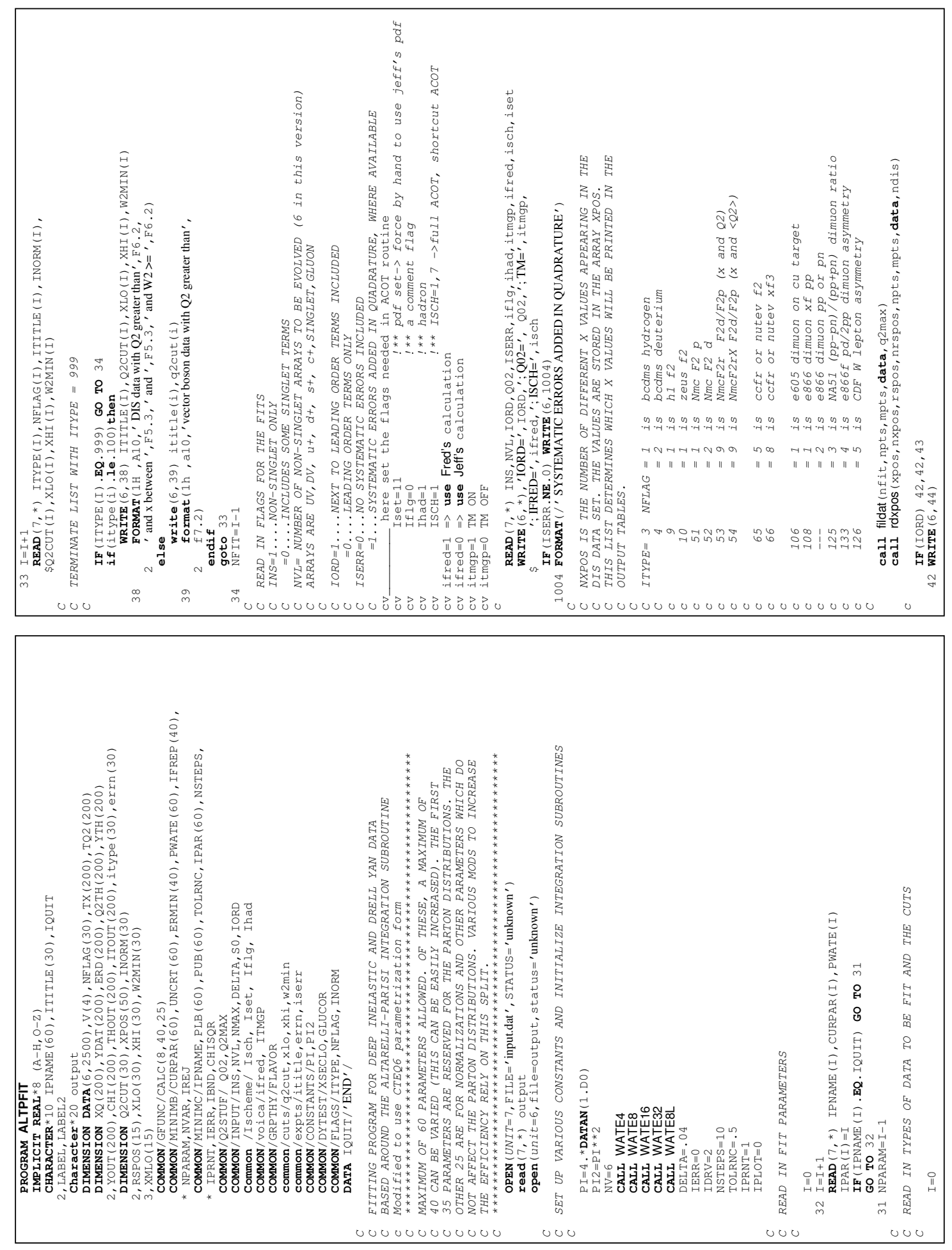

Figure D2: Main Program; pages 1-2. 

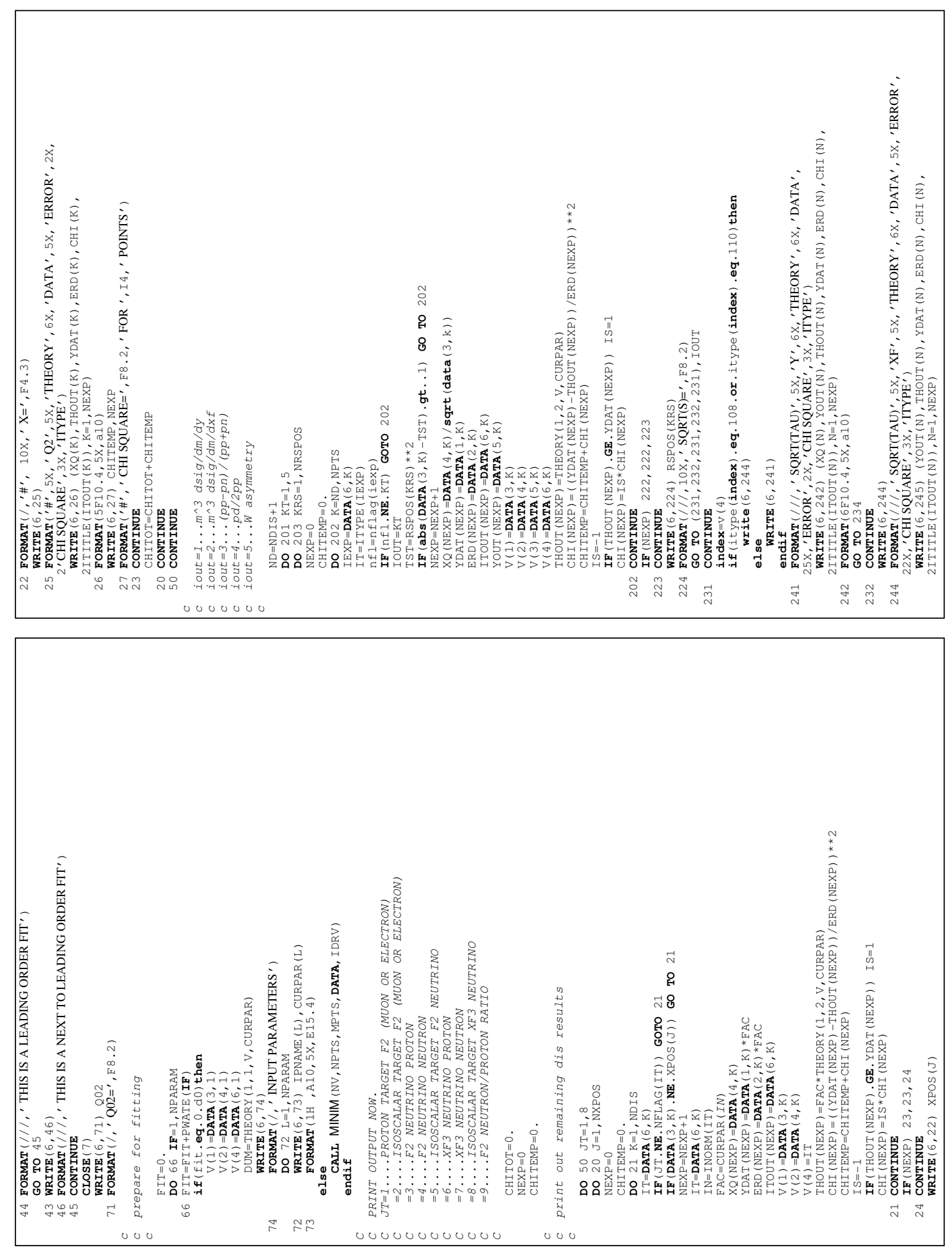

Figure D3: Main Program; pages 3-4. 

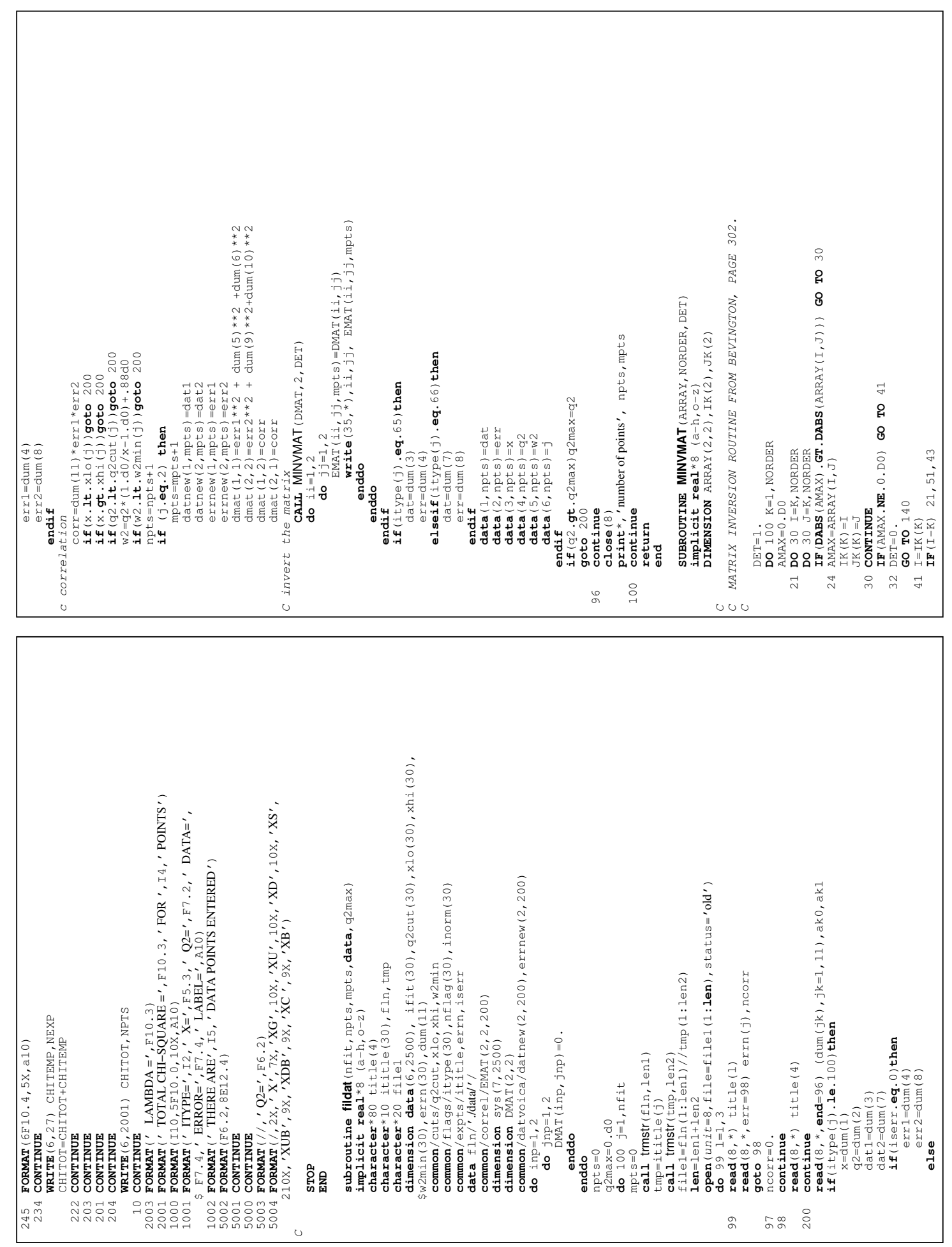

Figure D4: Main Program; pages 5-6. 

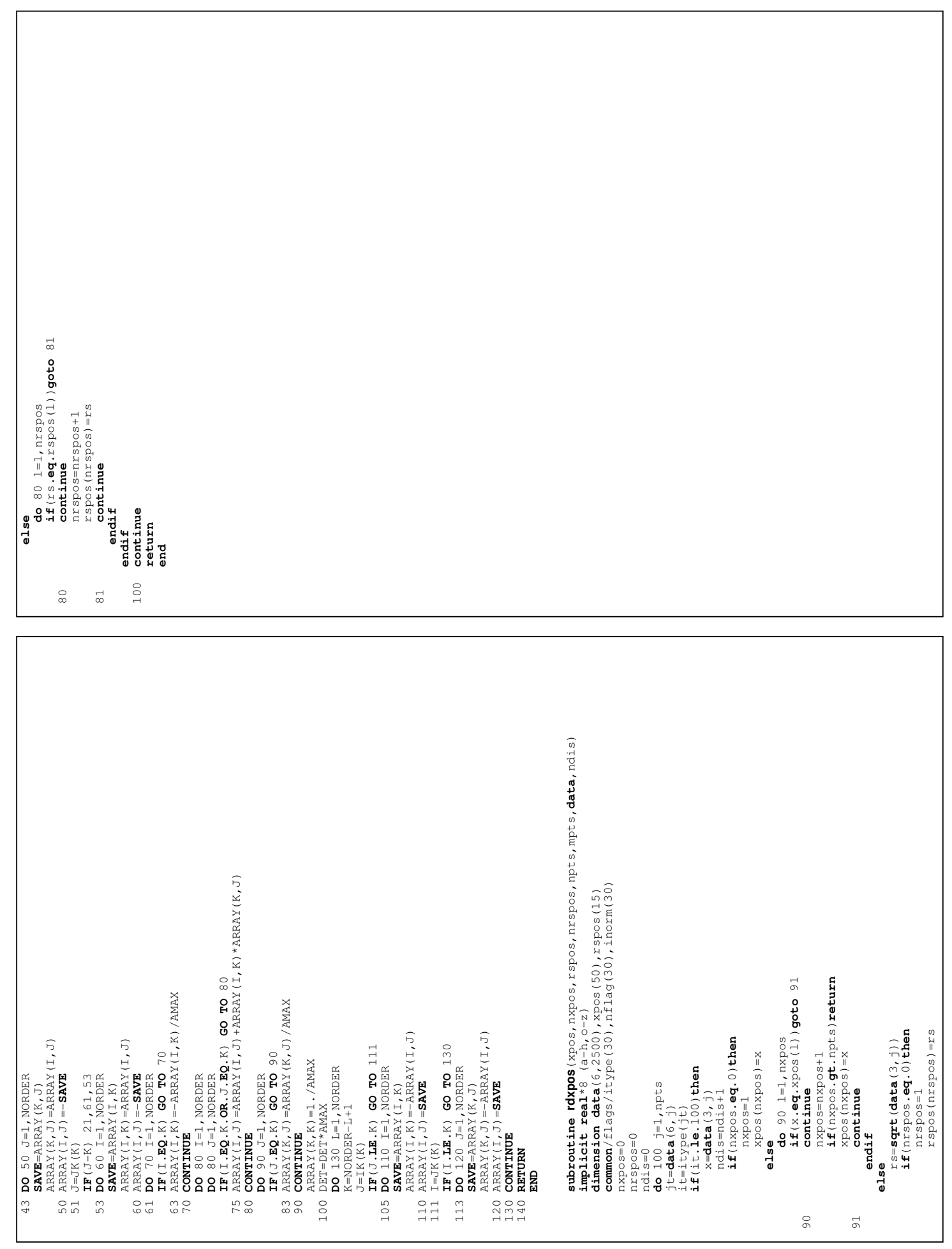

Figure D5: Main Program; pages 7-8. 

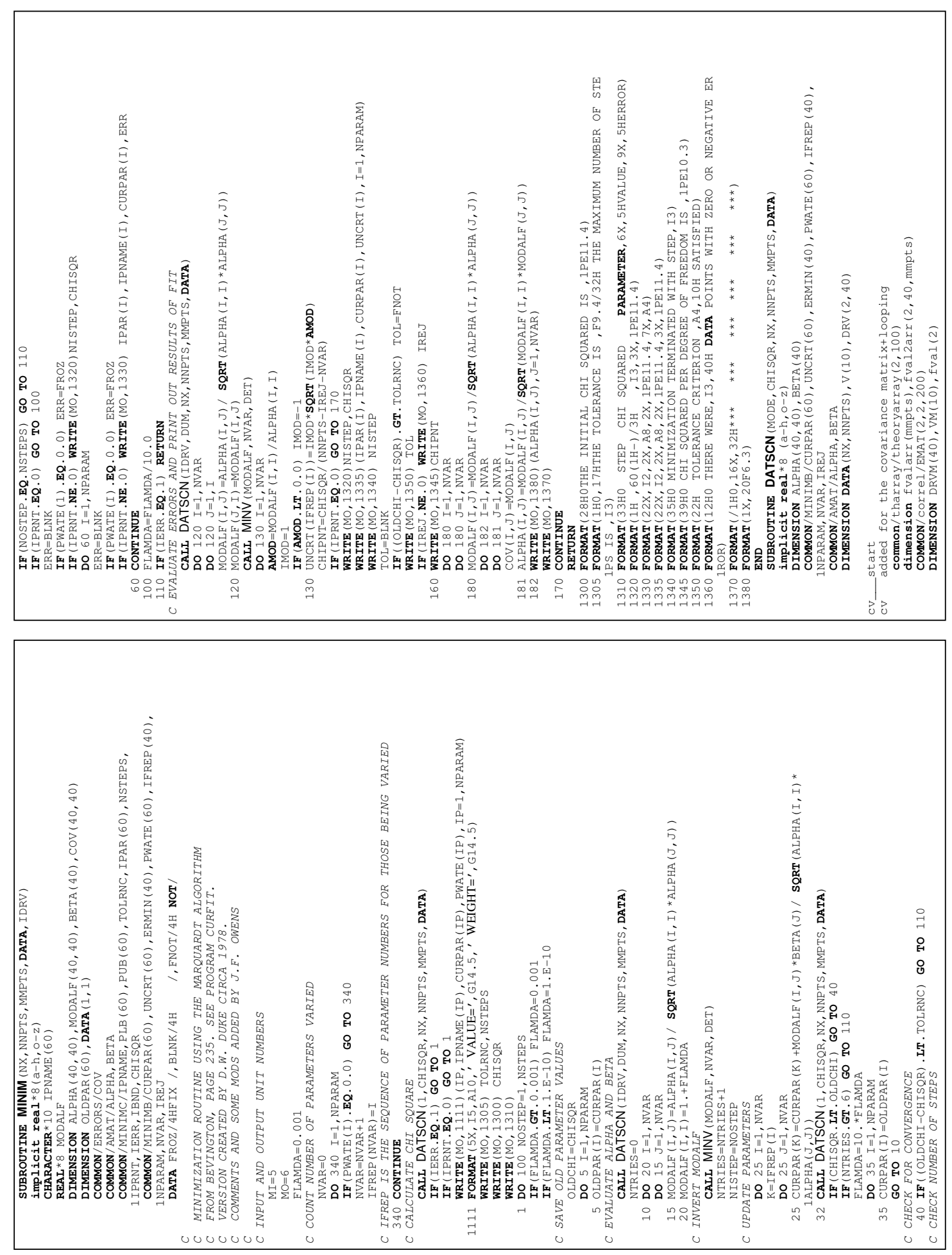

Figure D6: Minimization Program; pages 1-2. 

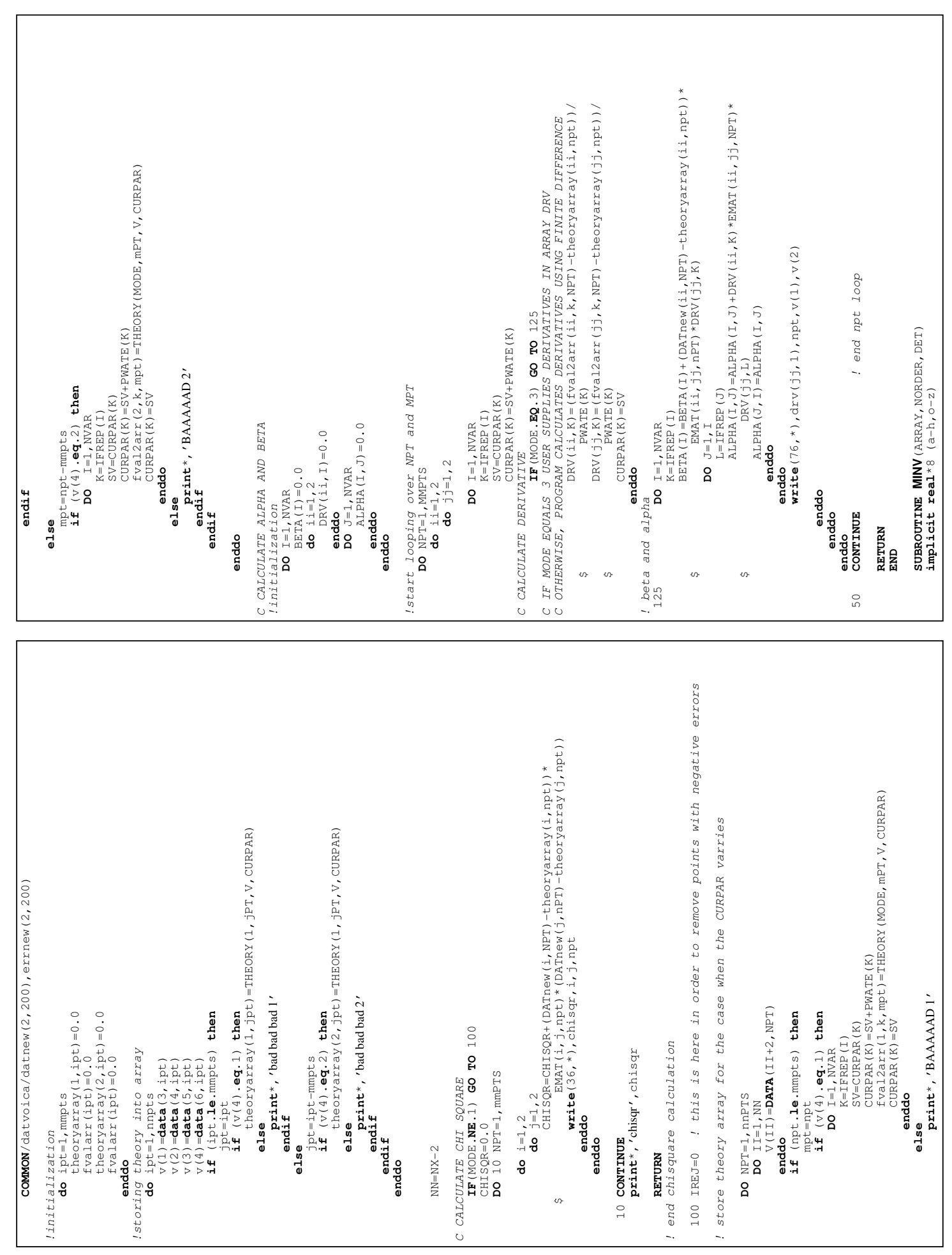

Figure D7: Minimization Program; pages 3-4. 


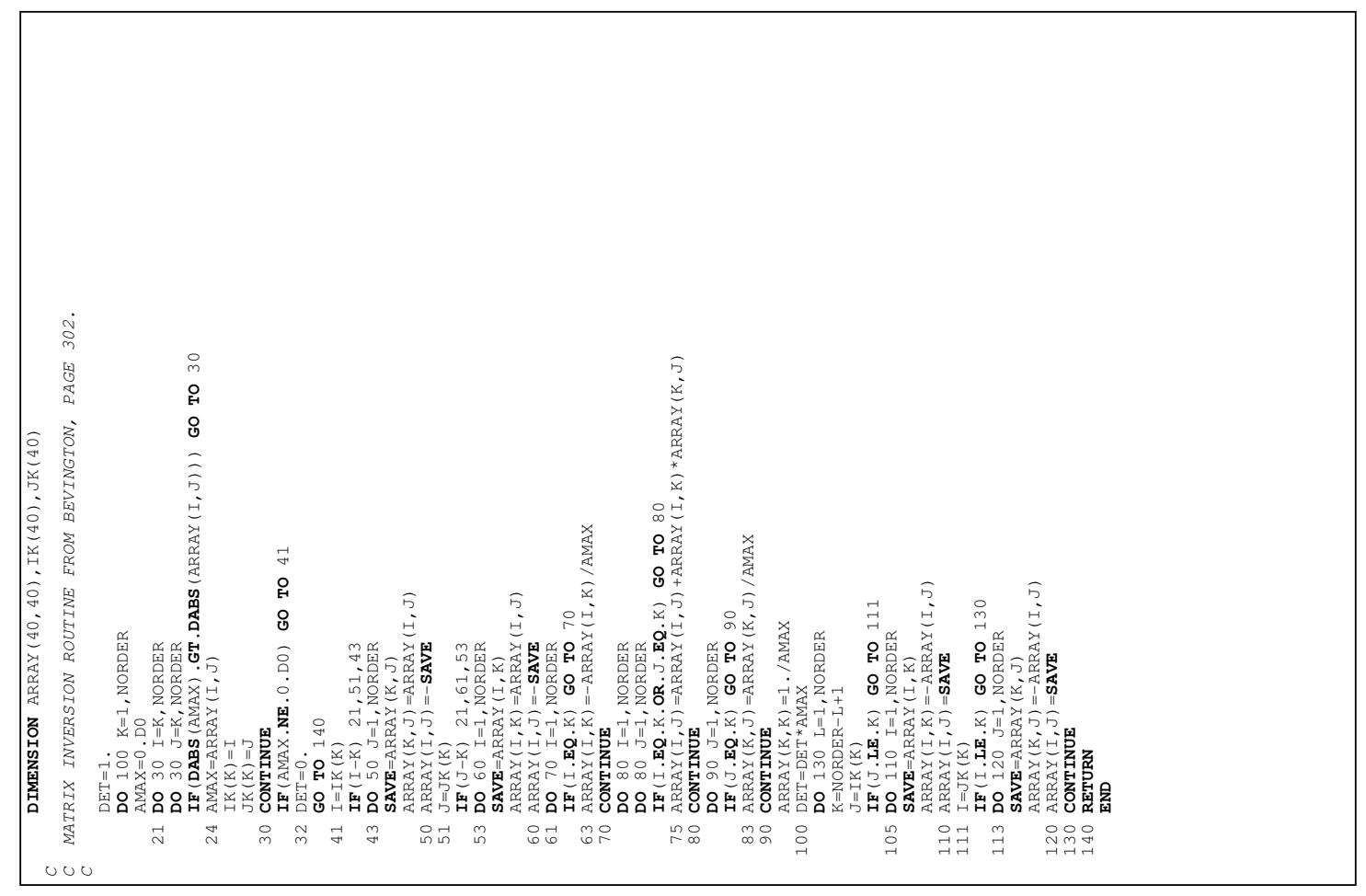

Figure D8: Minimization Program; pages 5-6. 

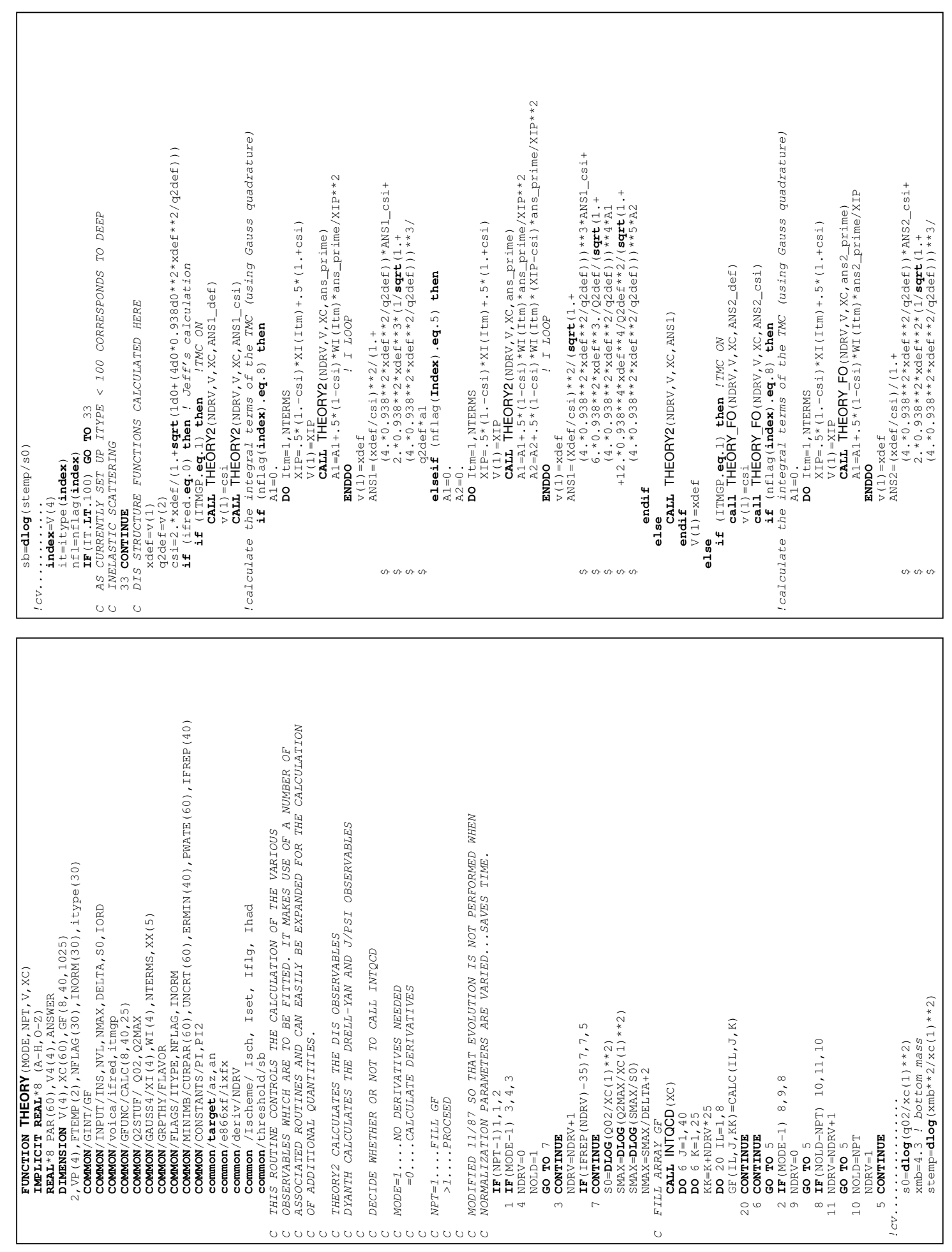

Figure D9: Theory $\overline{M S}$ Program; pages 1-2. 

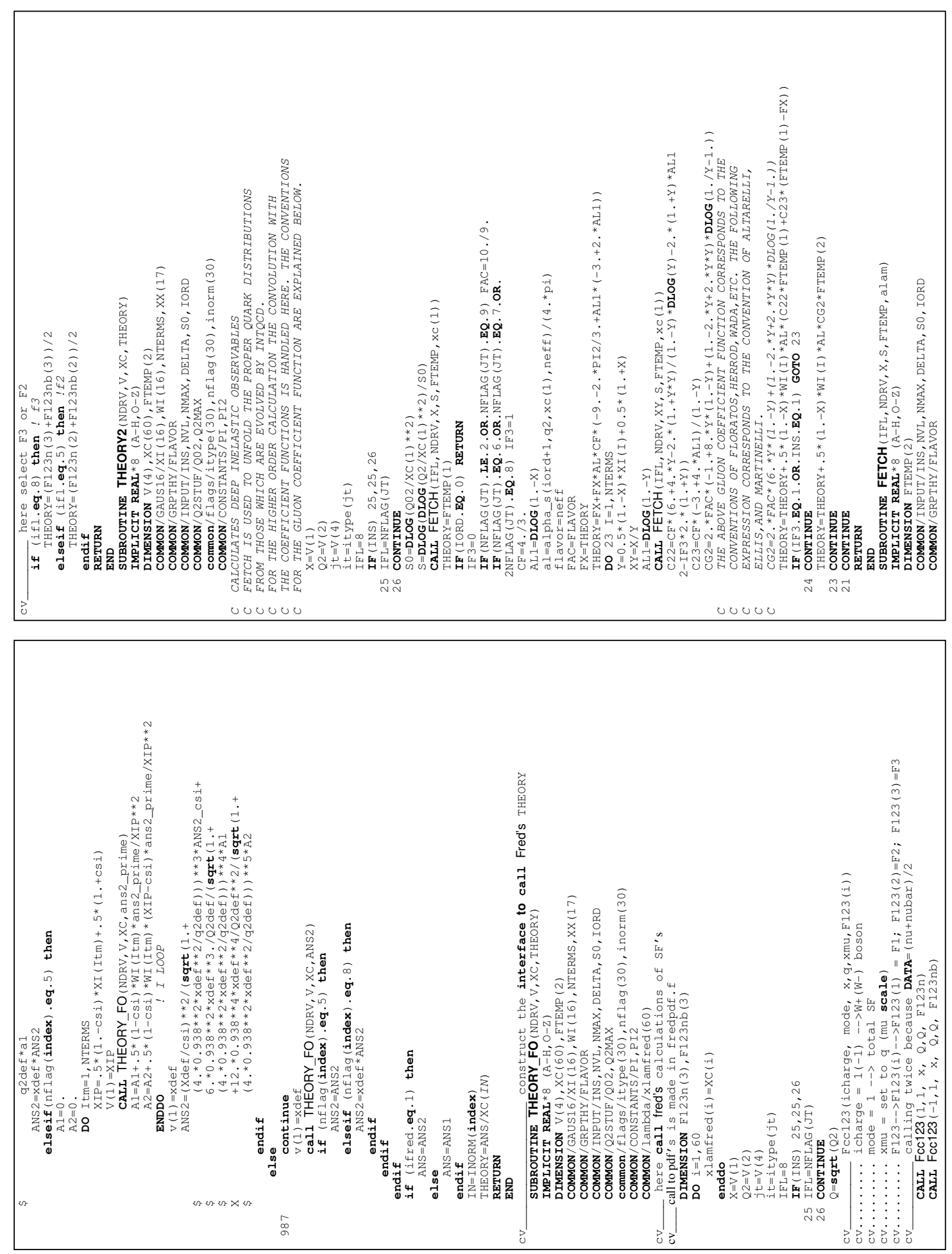

Figure D10: Theory $\overline{M S}$ Program; pages 3-4. 

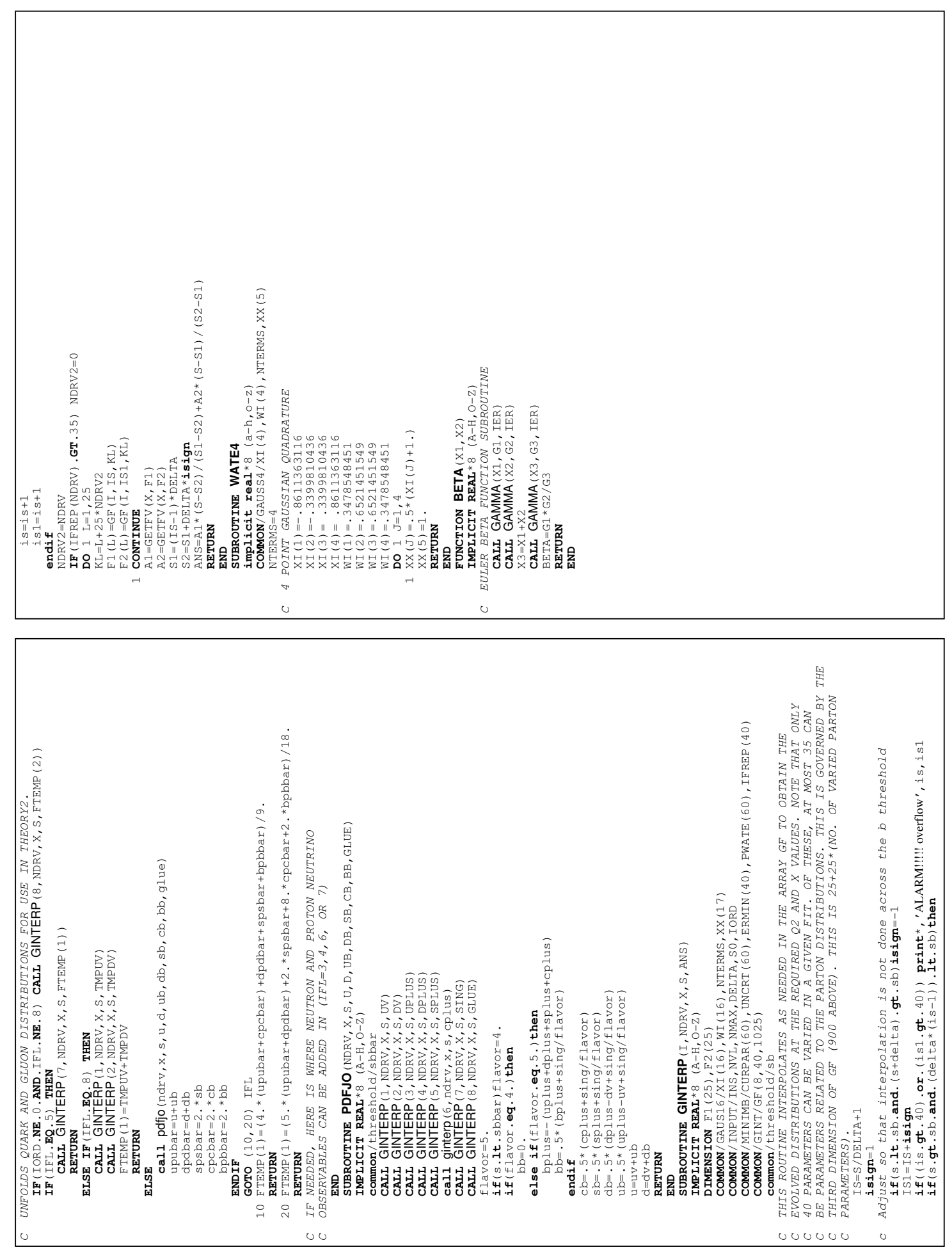

Figure D11: Theory $\overline{M S}$ Program; pages 5-6. 

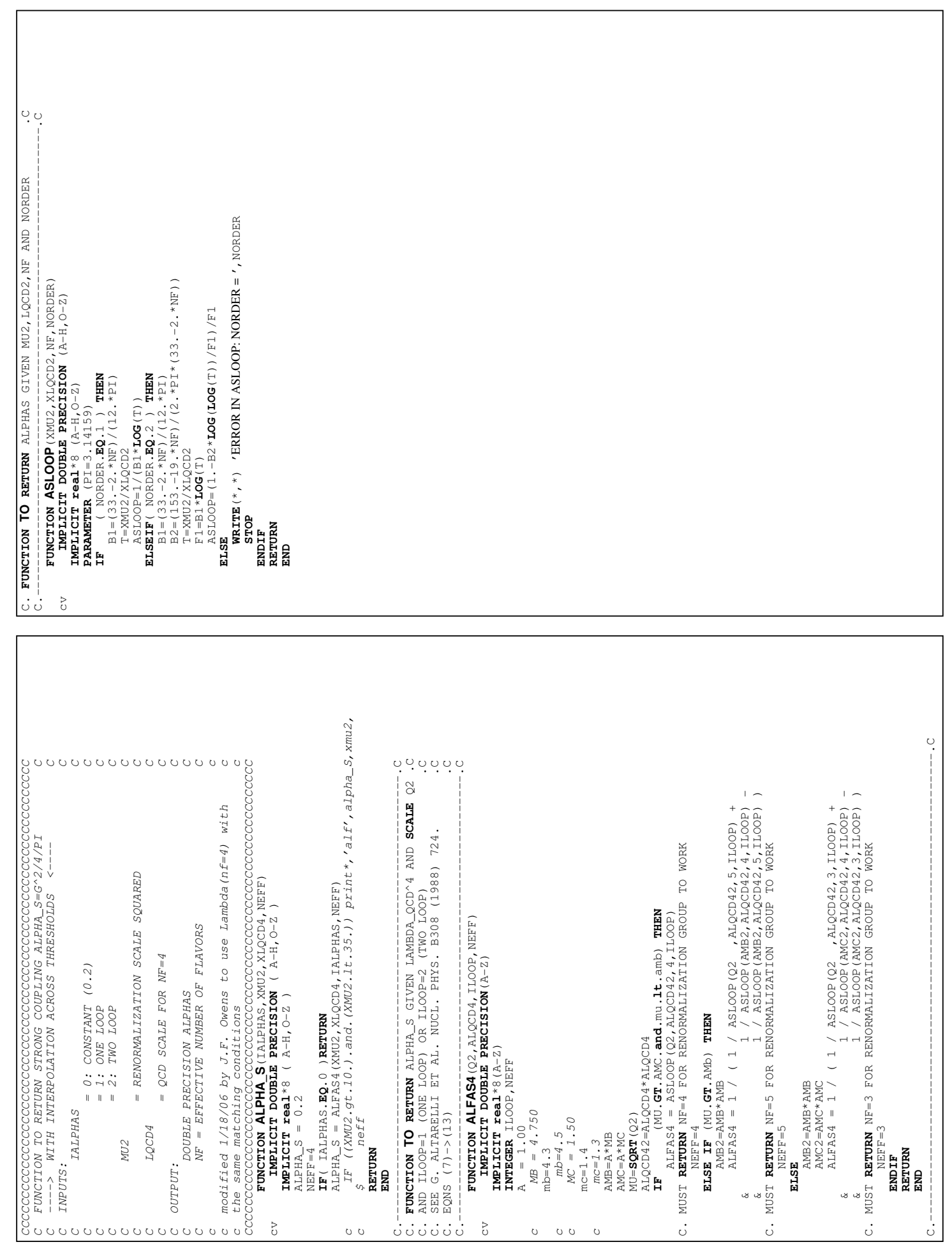

Figure D12: Alpha_S Program. 

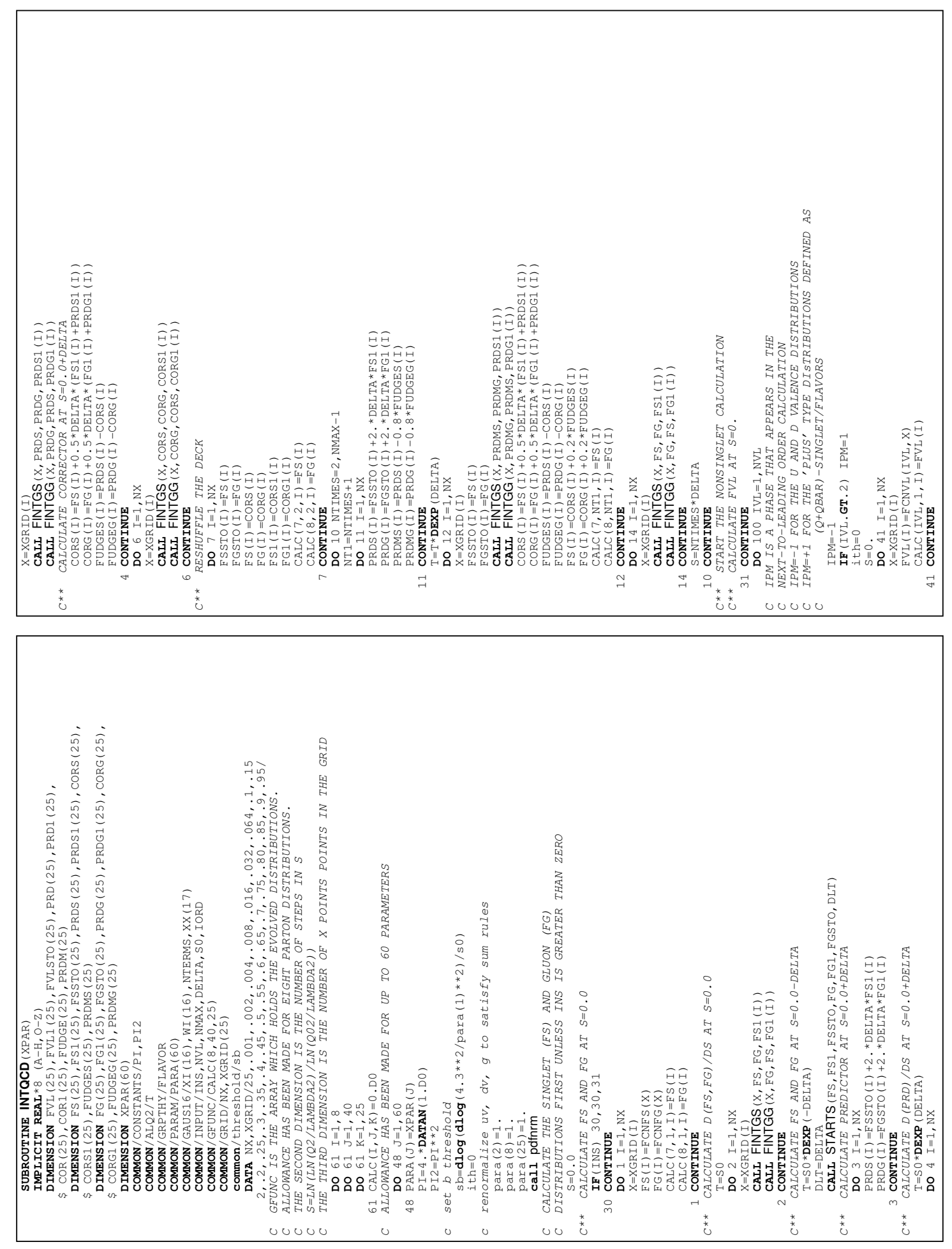

Figure D13: Evolution Code; pages 1-2. 

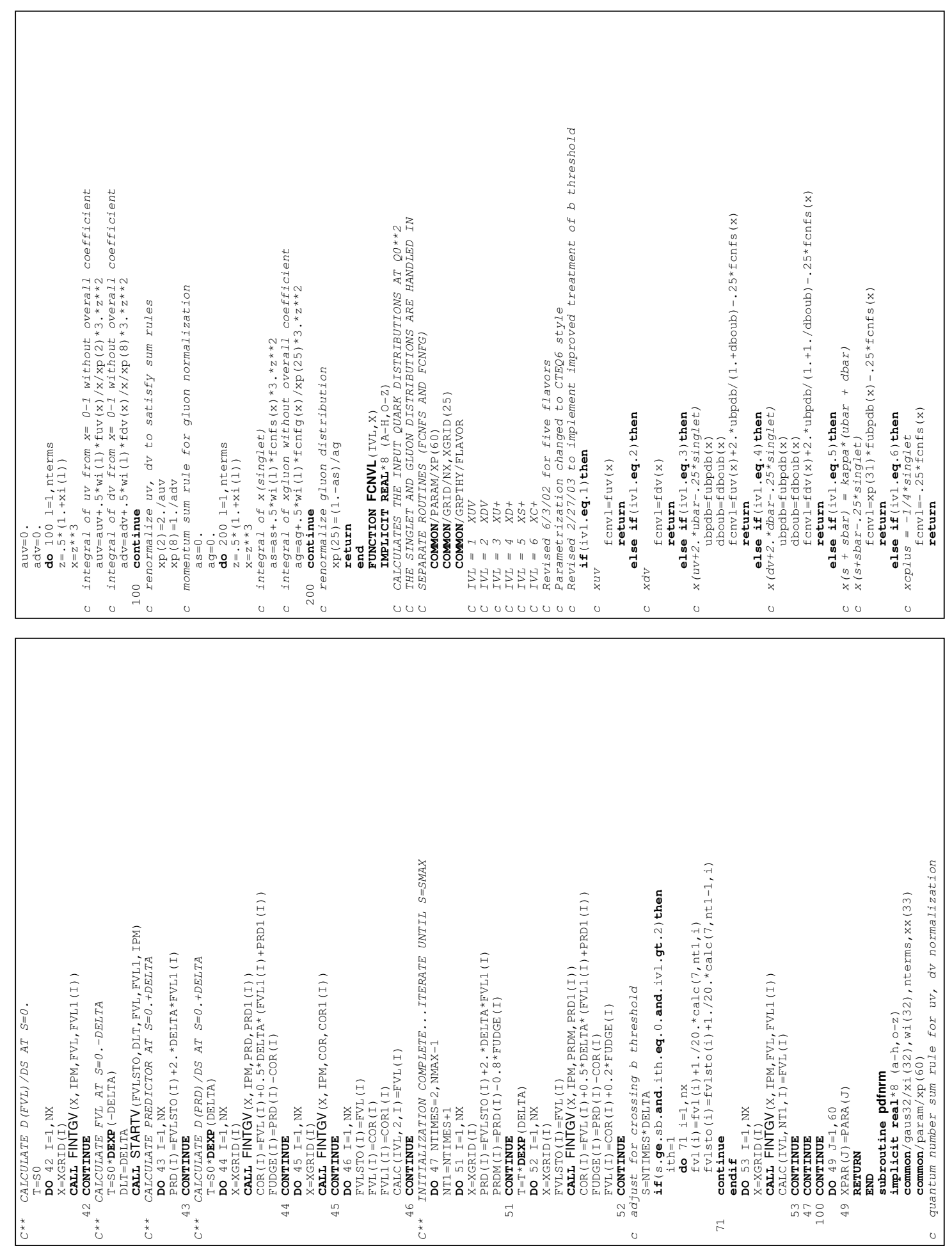

Figure D14: Evolution Code; pages 3-4. 

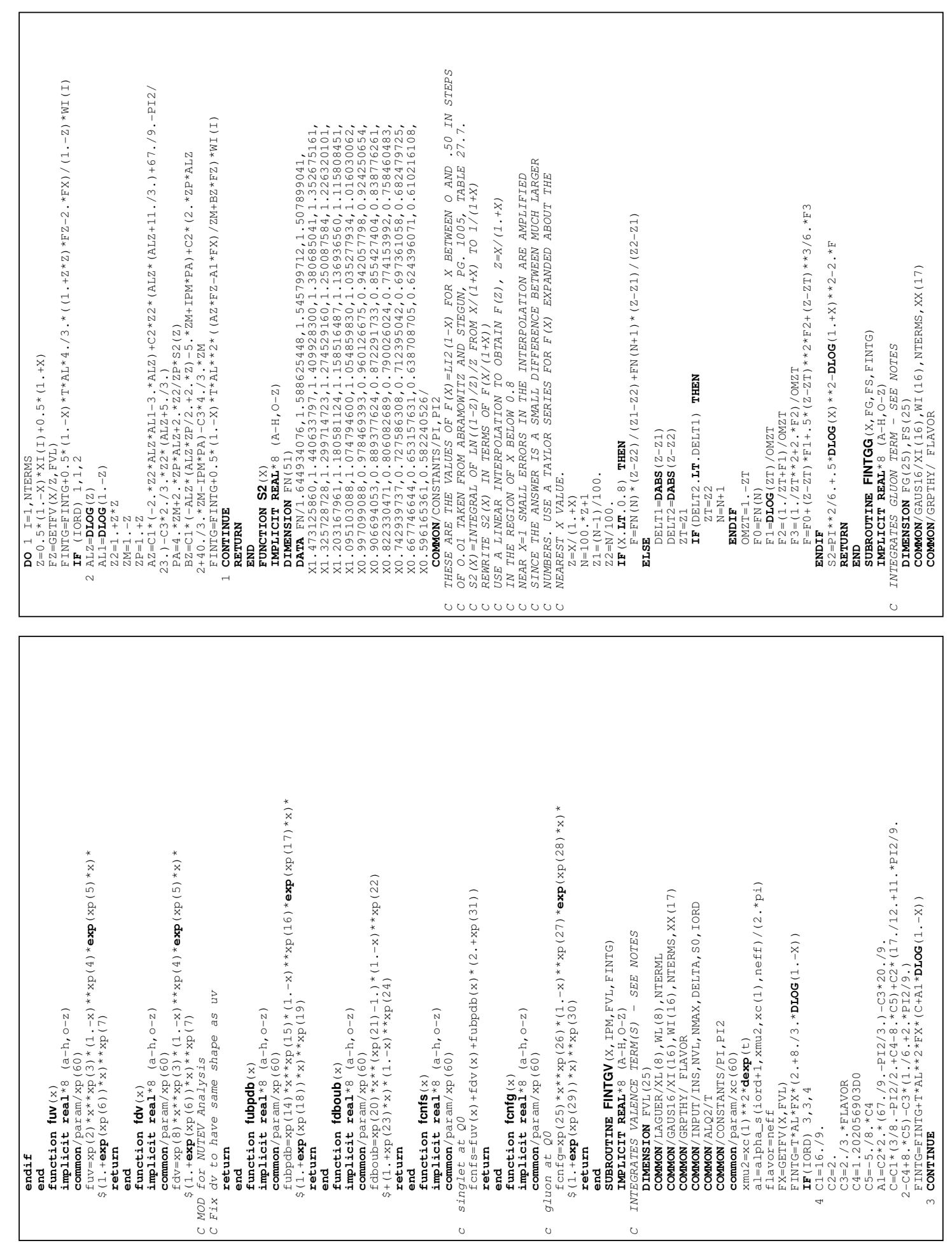

Figure D15: Evolution Code; pages 5-6. 

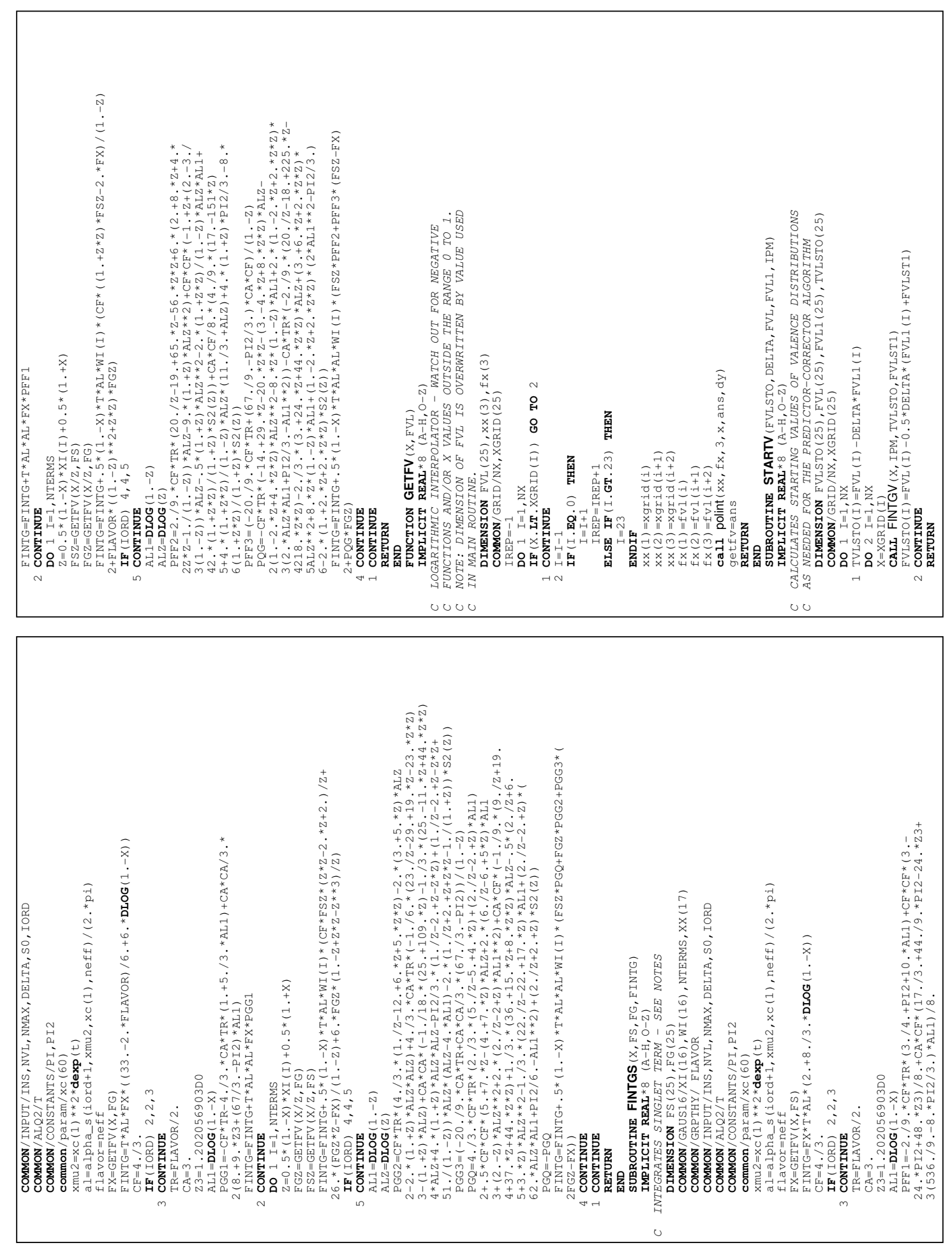

Figure D16: Evolution Code; pages 7-8. 

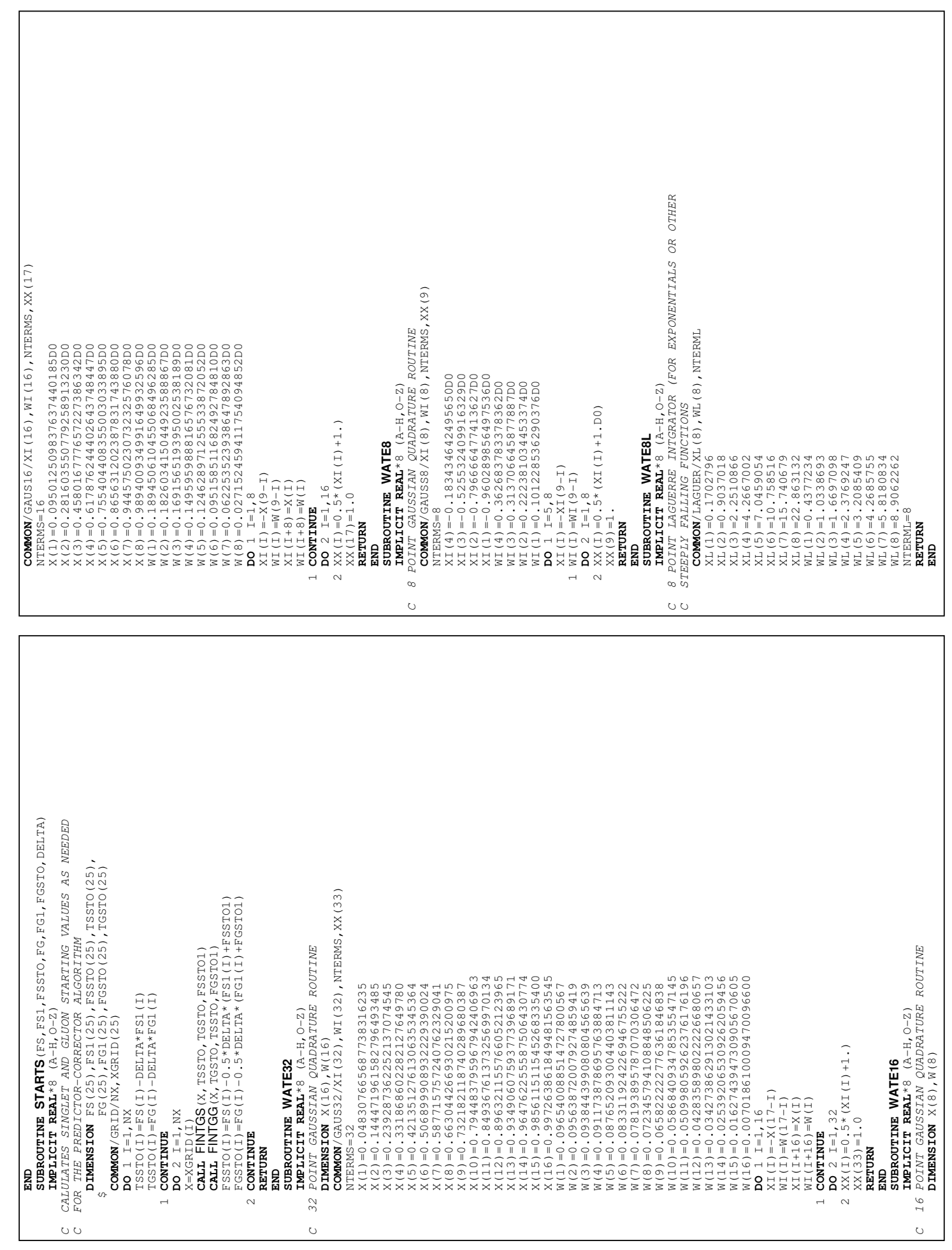

Figure D17: Evolution Code; page 9-10. 
APPENDIX E

QCD SOURCE CODE: PART B

This chapter presents the key programs which use the ACOT scheme [54]. 

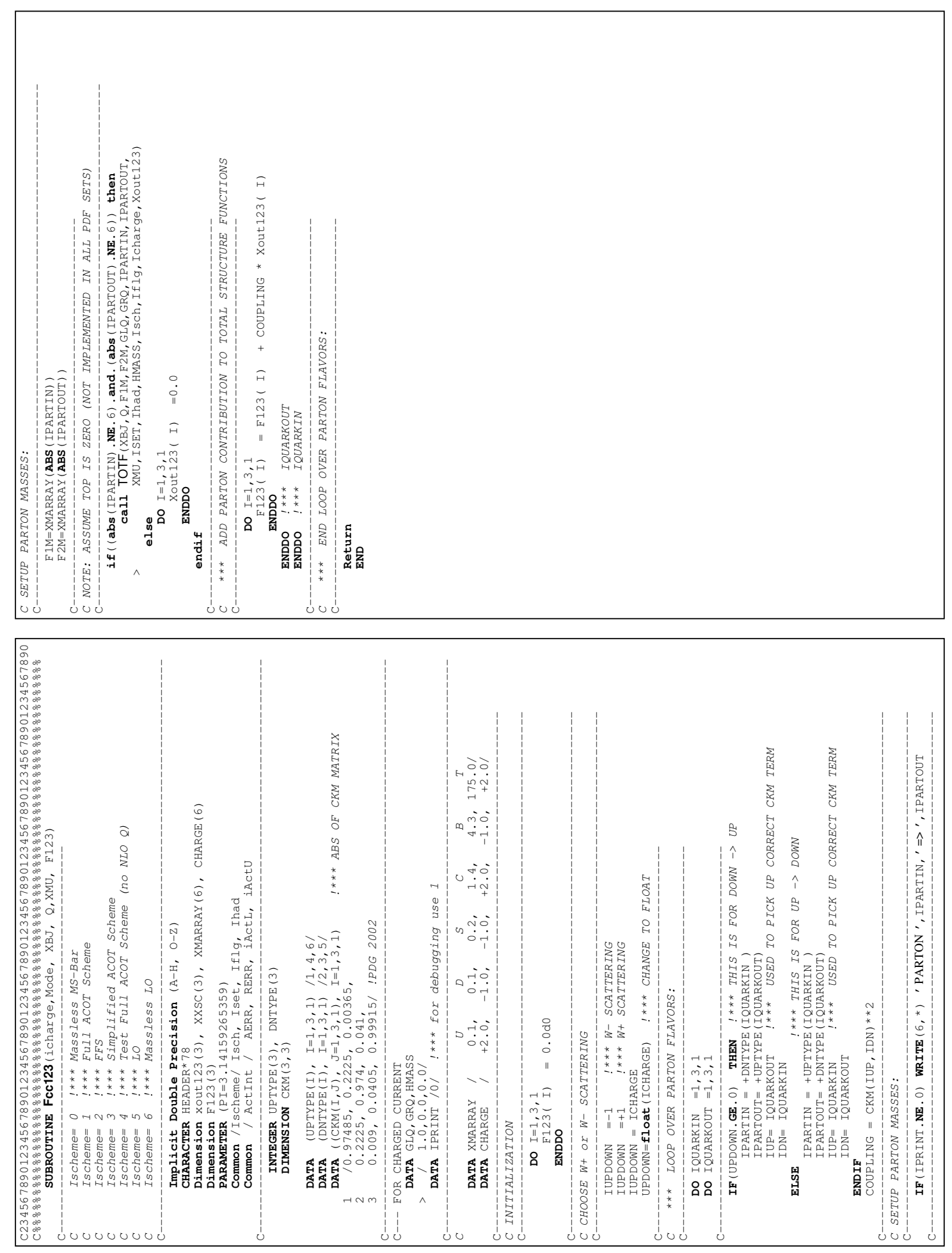

Figure E1: Constructing Structure functions using ACOT scheme . 

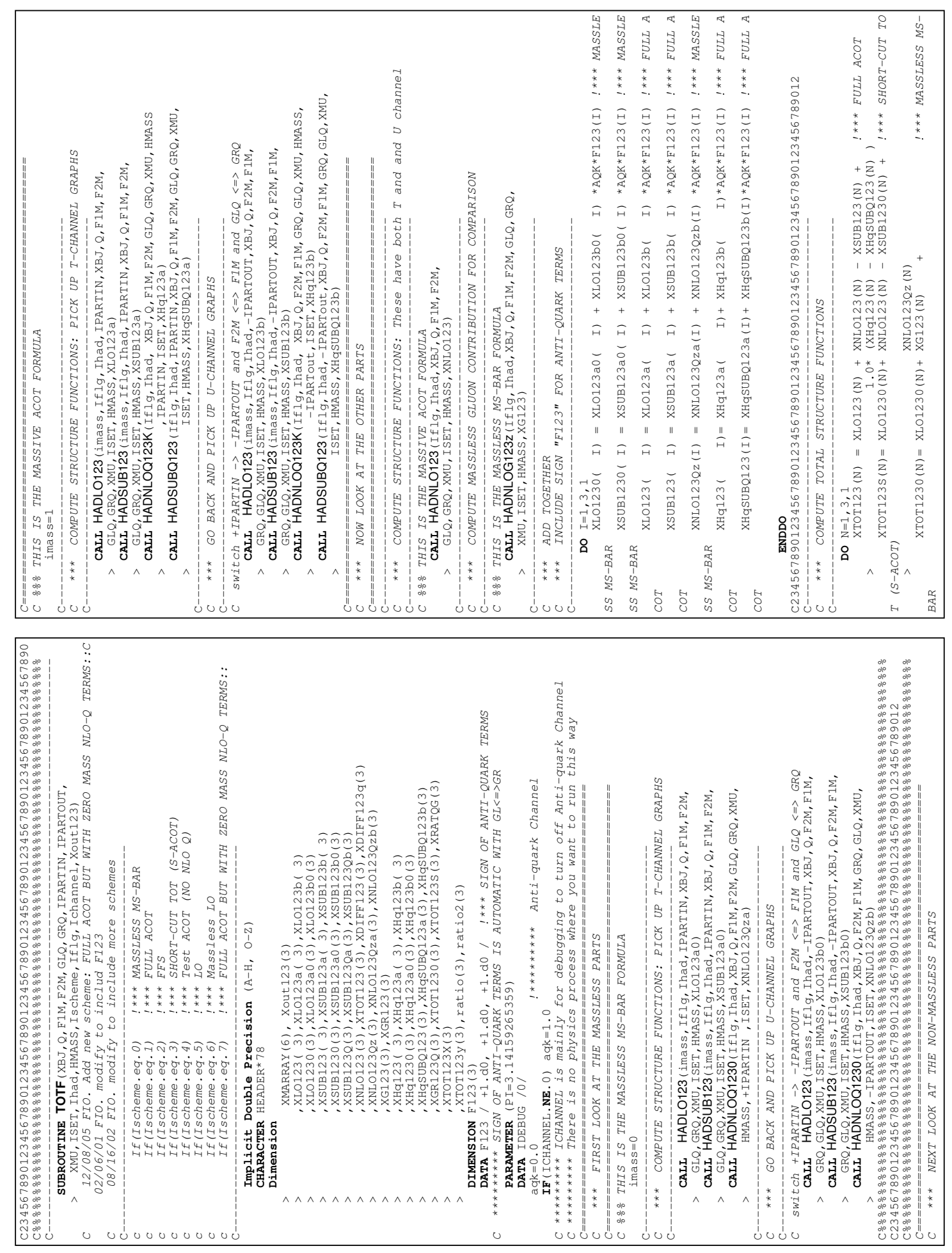

Figure E2: Program which selects ACOT scheme; pages 1-2. 


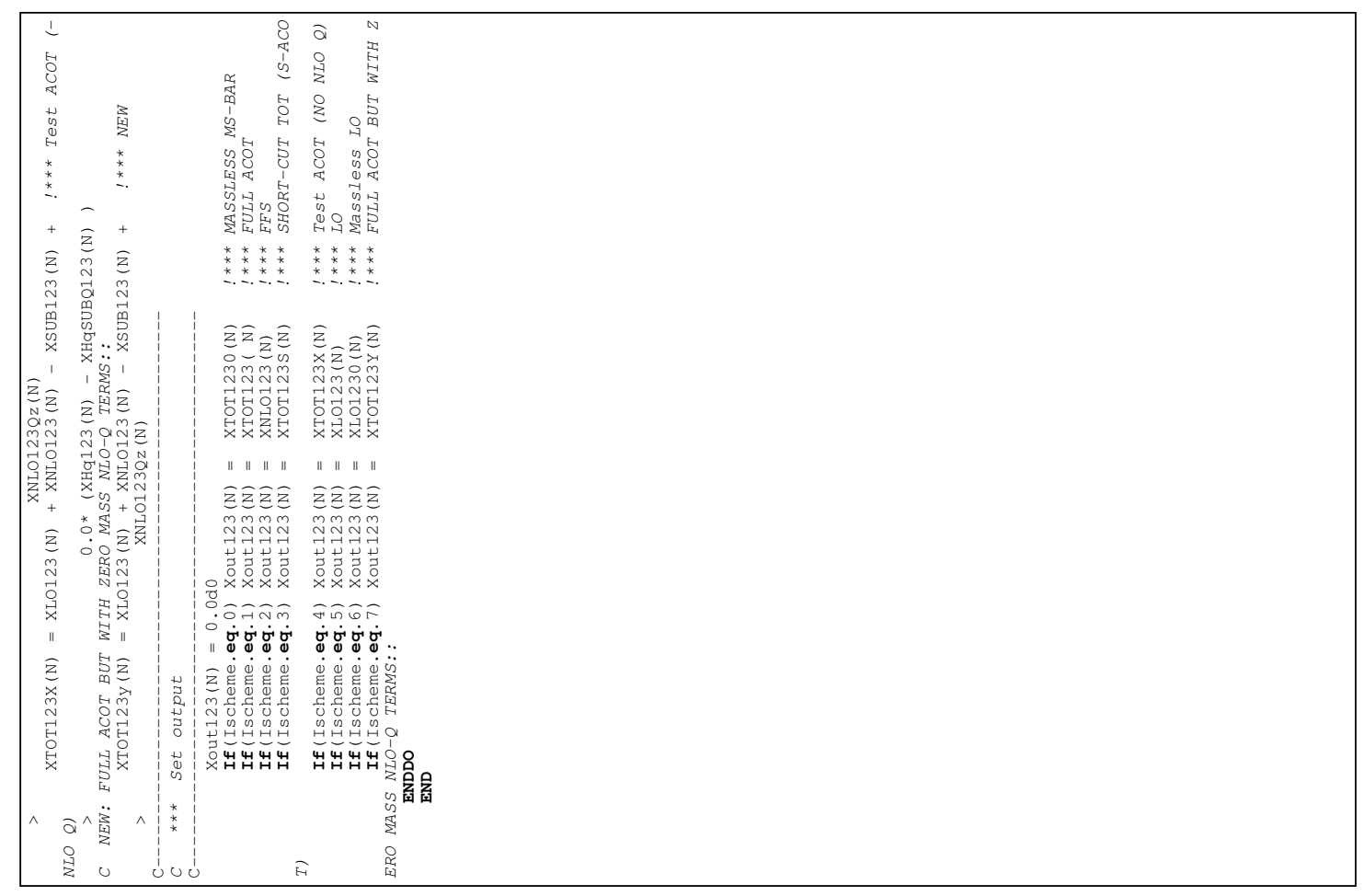

Figure E3: Program which selects ACOT scheme;pages 3-4. 

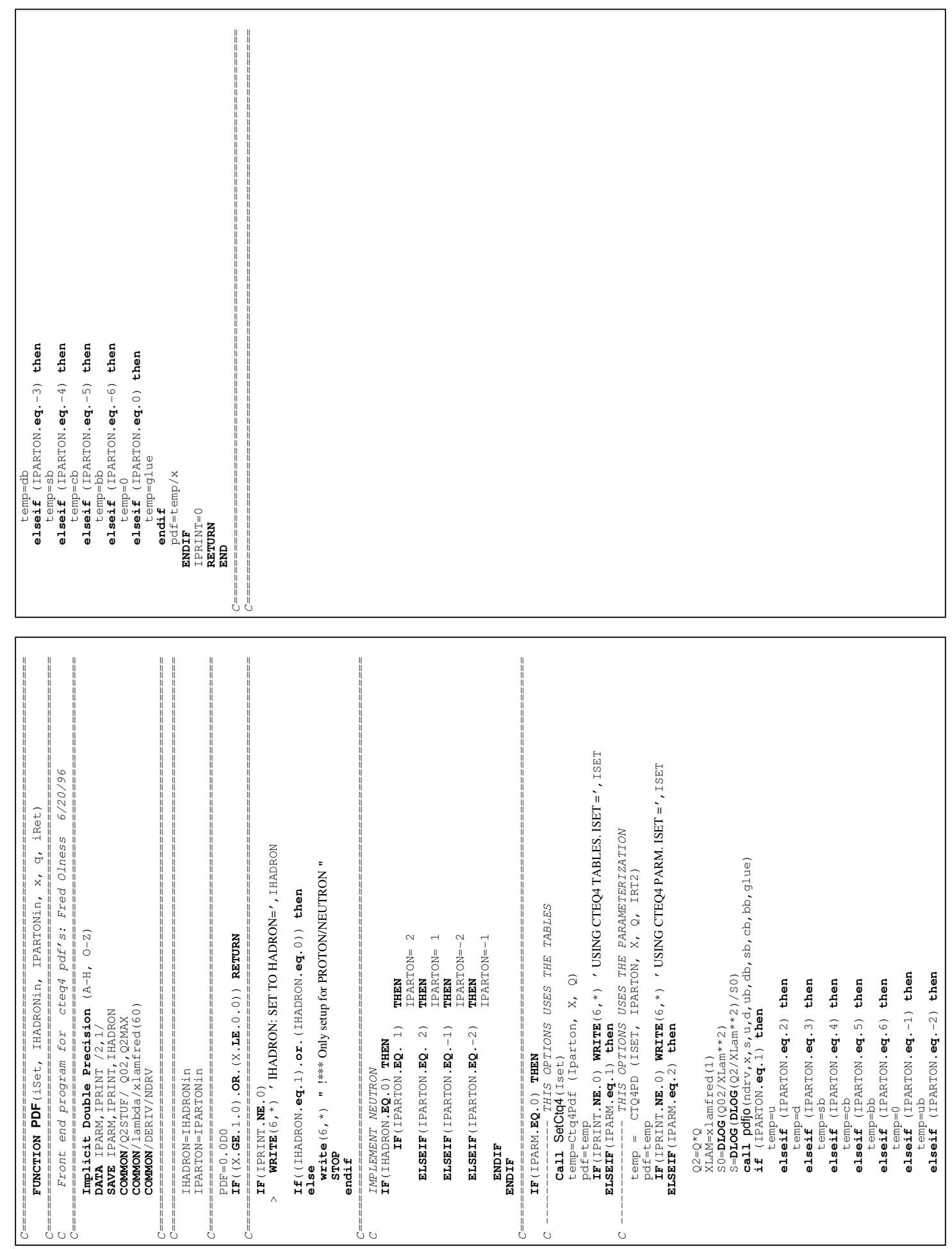

Figure E4: ACOT program which calls the parametrized PDFs. 


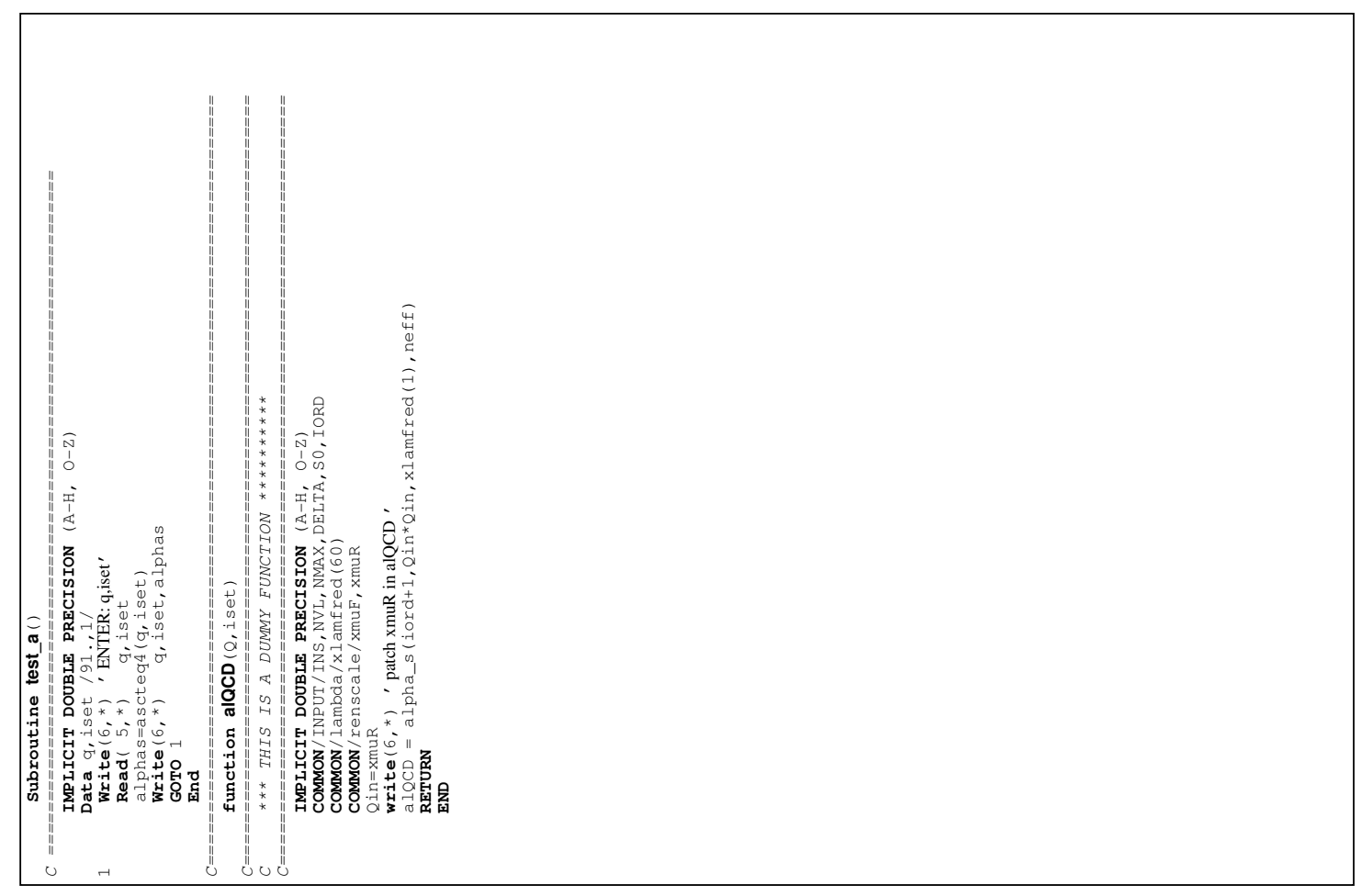

Figure E5: ACOT program calling $\alpha_{S}$. 

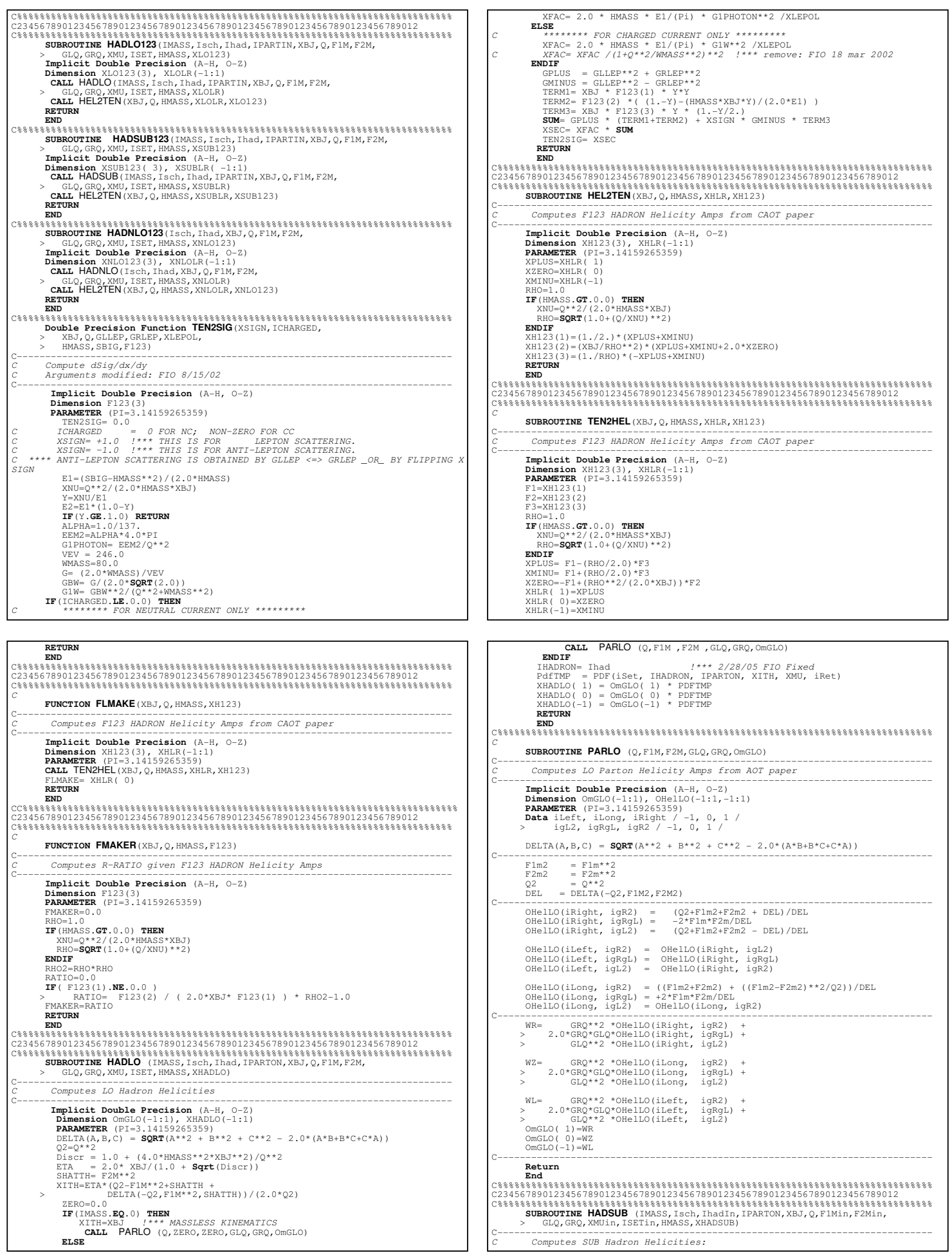

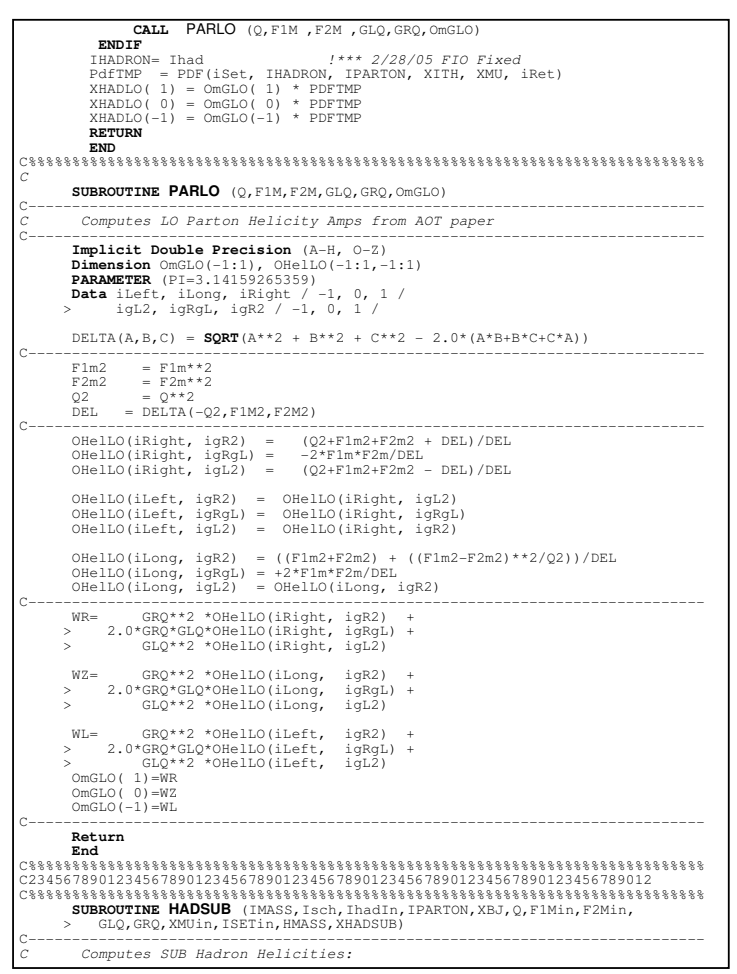

Figure E6: Program which computes each diagram; pages 1-4. 



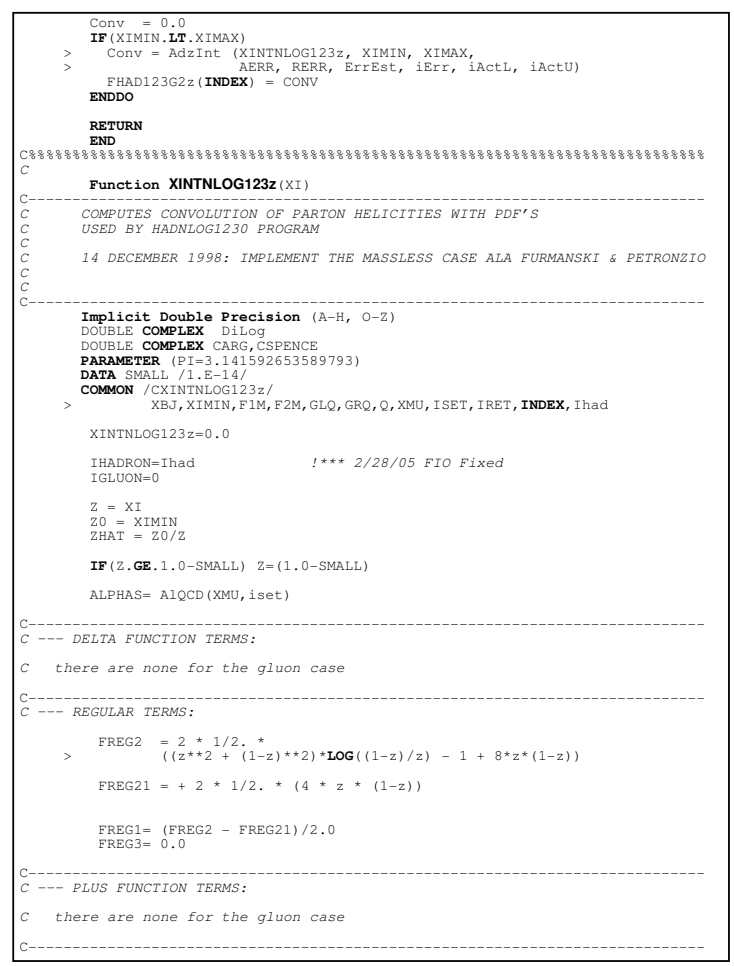

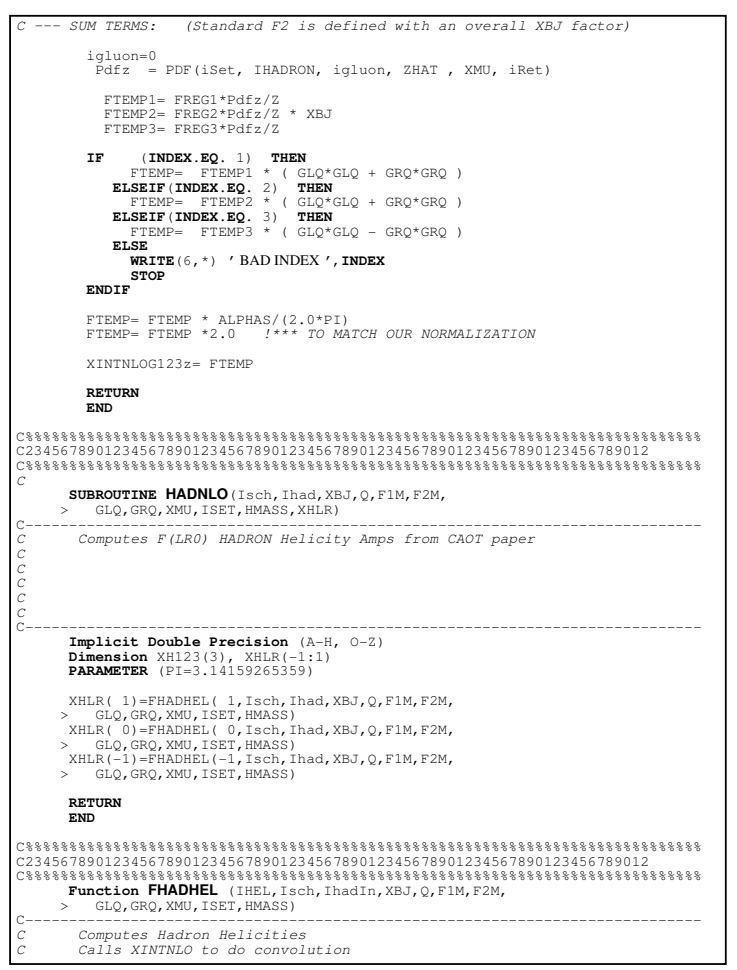

Figure E7: Program which computes each diagram; pages 5-8. 

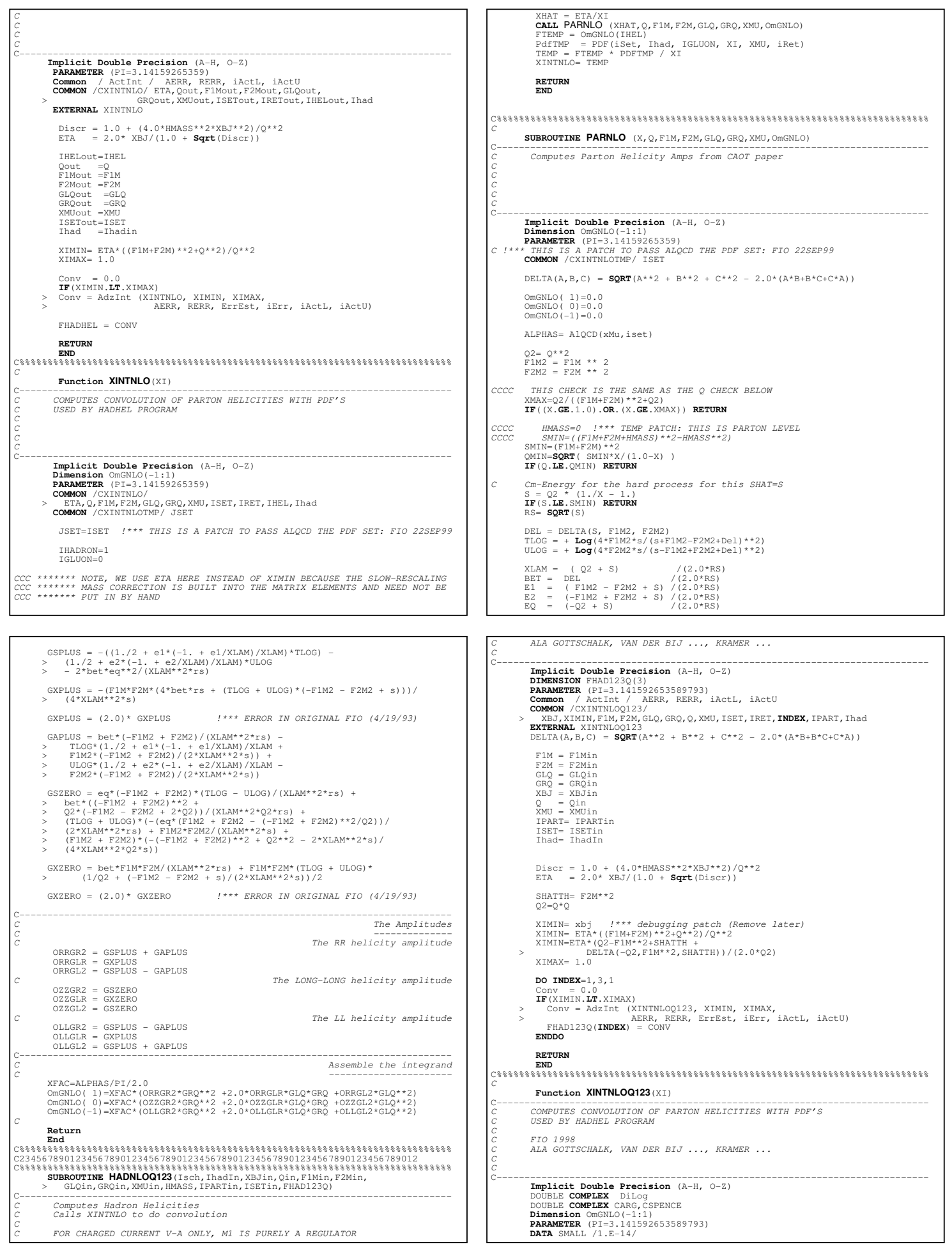

Figure E8: Program which computes each diagram; pages 9-12. 


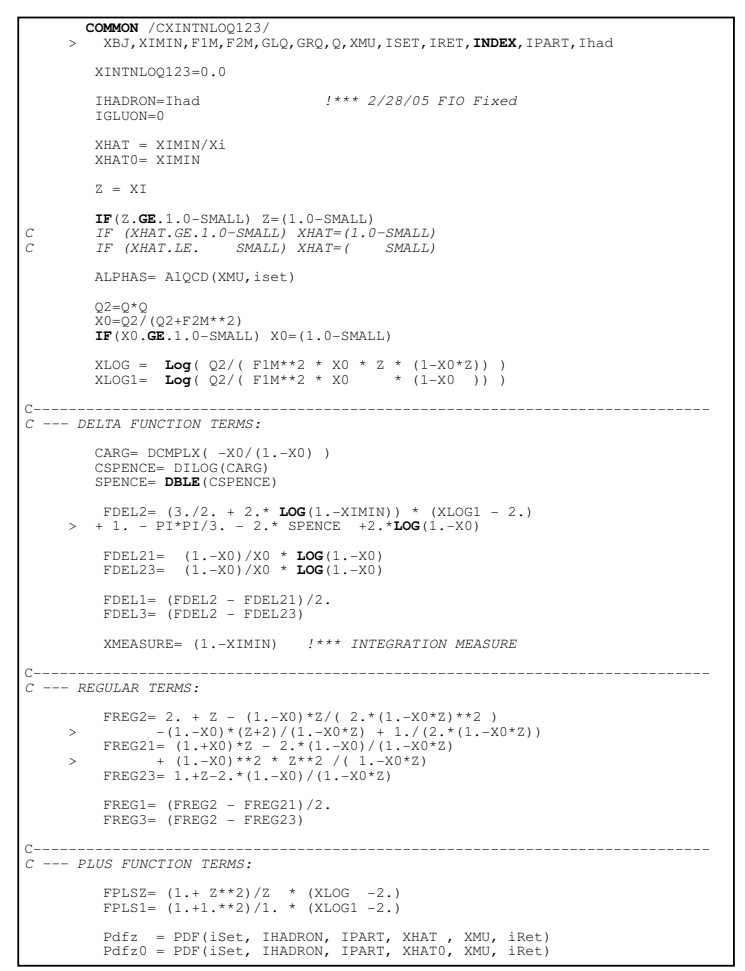

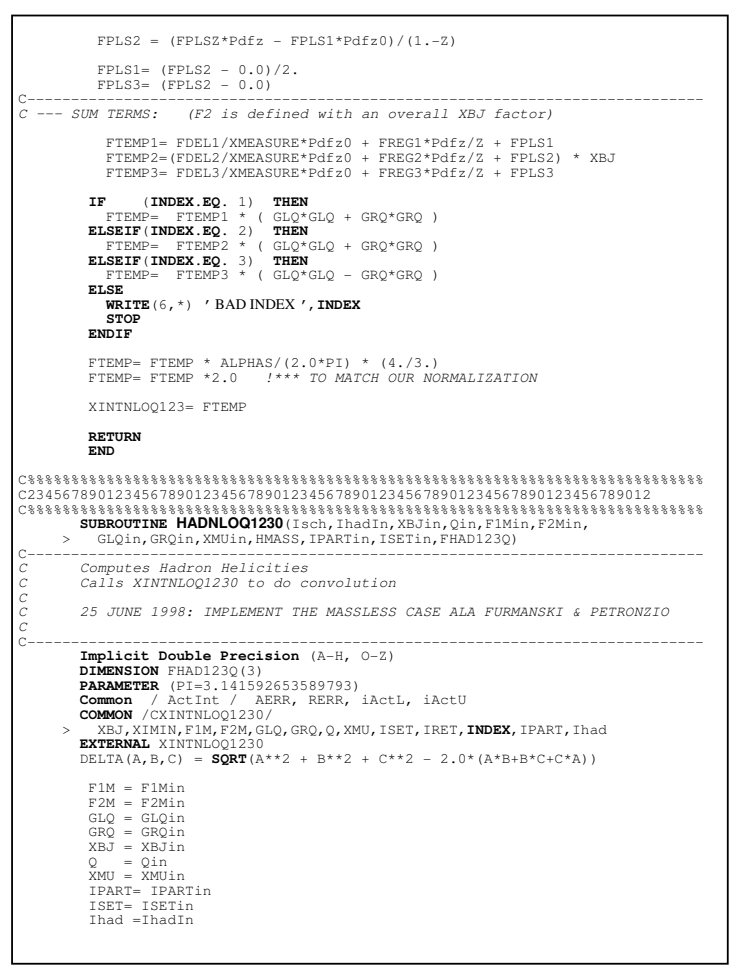

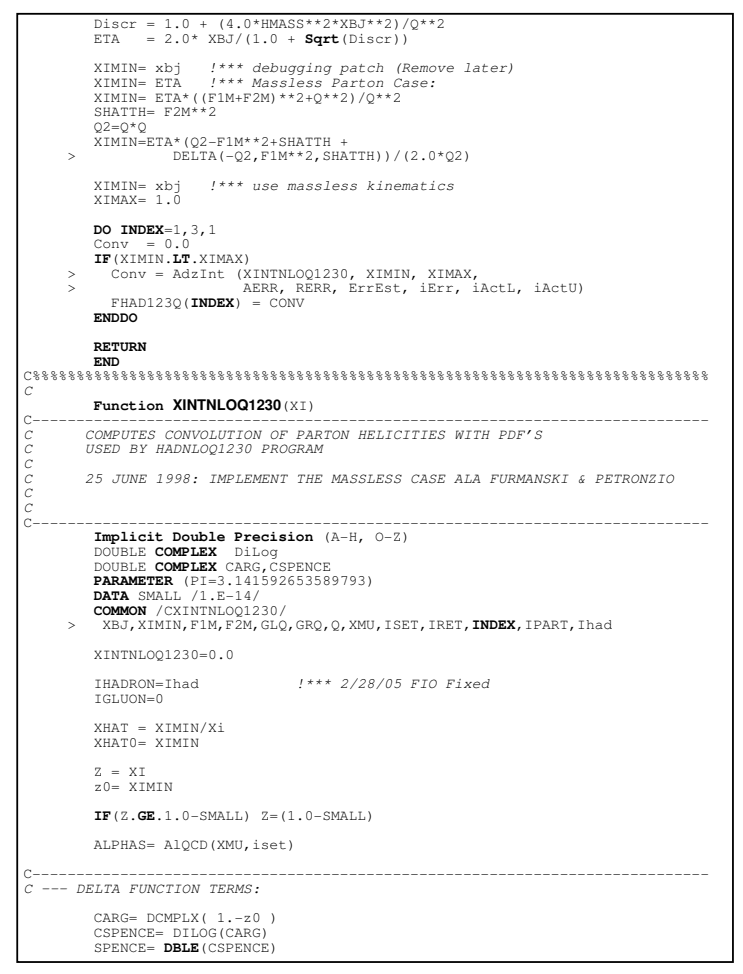

\begin{tabular}{|c|}
\hline 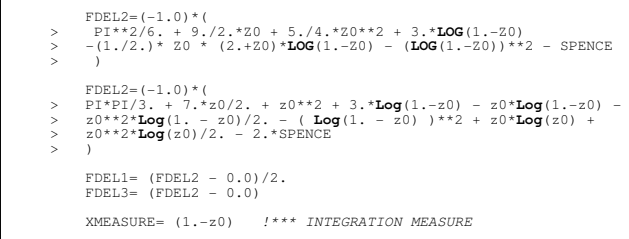 \\
\hline 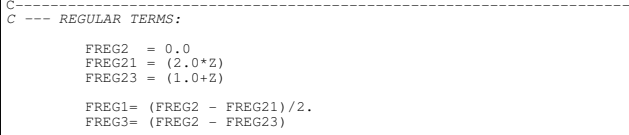 \\
\hline  \\
\hline 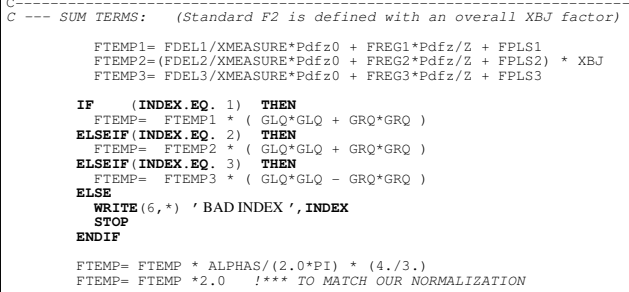 \\
\hline
\end{tabular}

Figure E9: Program which computes each diagram; pages 13-16. 


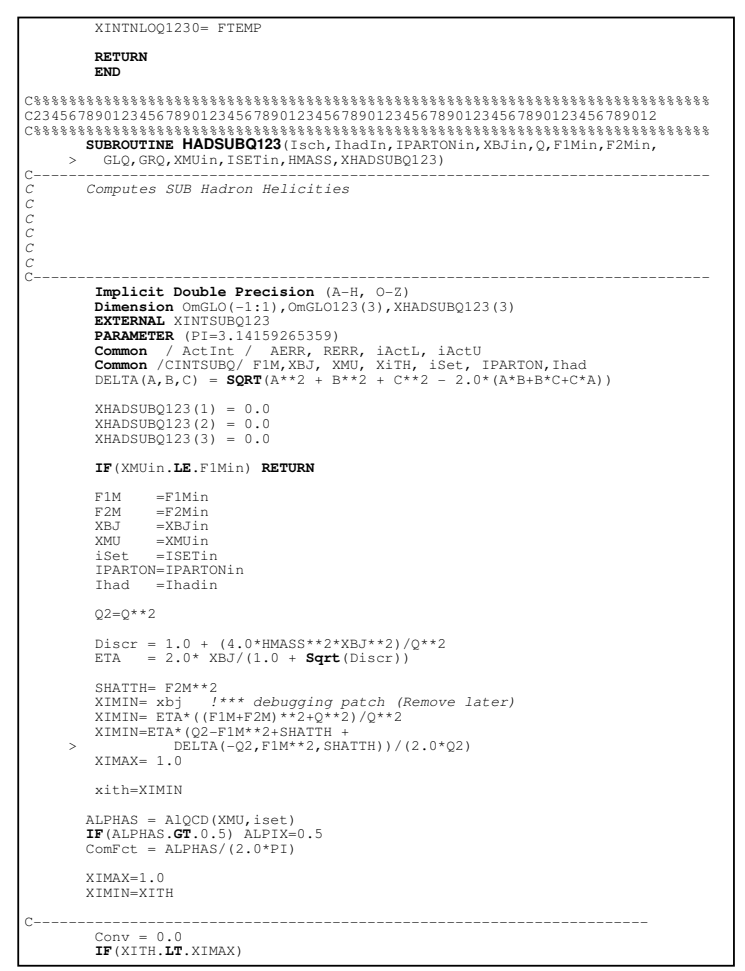

\begin{tabular}{|c|}
\hline 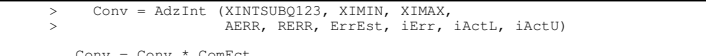 \\
\hline ConV = CONV . COMIRE $\mathrm{CL}$ \\
\hline CALL PARLO (Q, F1M, E2M, GLQ, GRQ, OMGLO) \\
\hline$X F A C=1 . /(1 .+(F 2 M / Q) * * 2)$ \\
\hline 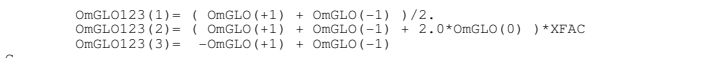 \\
\hline 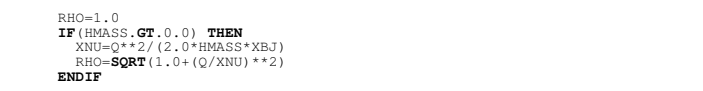 \\
\hline 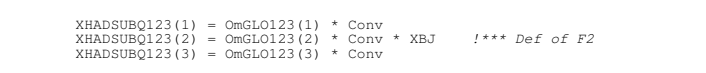 \\
\hline$\underset{\substack{R E T U R N \\
E N D}}{\mathrm{R}}$ \\
\hline 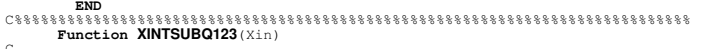 \\
\hline C The splitting function for $Q^{->Q}$ \\
\hline $\begin{array}{l}\text { Implicit Double Precision (A-H, } 0-Z) \\
\text { DATA SMALL } / 1 \text {. E-14/ }\end{array}$ \\
\hline $\begin{array}{l}\text { > Common } \\
\text { F1Min, XBNin, XMUIN, XiTHIN, iSetIN, IPARTONin, IhadIn }\end{array}$ \\
\hline 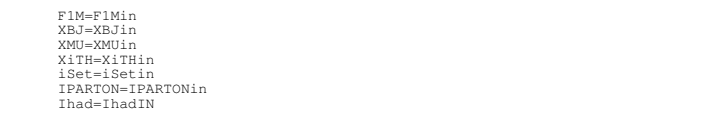 \\
\hline $\begin{array}{l}x=X \text { in } \\
\mathbf{I F}(x, G E, 1.0-\text { SMALL }) \quad X=1,0-\text { SMALL }\end{array}$ \\
\hline 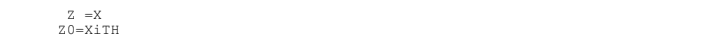 \\
\hline$C--C^{C--F^{\prime} S}$ \\
\hline 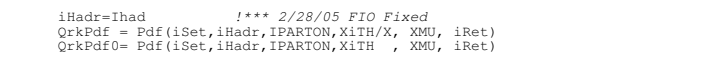 \\
\hline C--- DELTA FUNCTION TERMS: \\
\hline
\end{tabular}

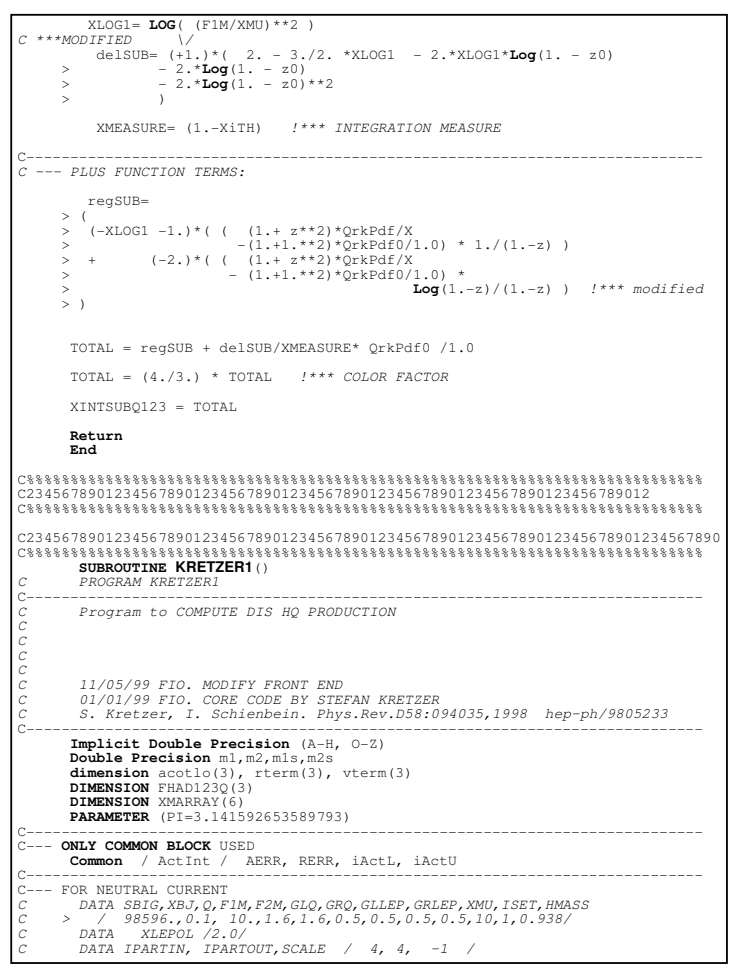

\begin{tabular}{|c|c|}
\hline \multicolumn{2}{|r|}{  } \\
\hline \multicolumn{2}{|r|}{ 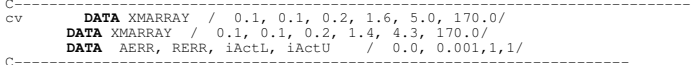 } \\
\hline \multicolumn{2}{|c|}{ C SETUP INTEGRATION PARAMETERS } \\
\hline \multicolumn{2}{|r|}{ 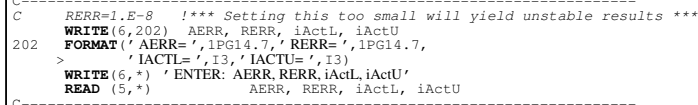 } \\
\hline \multicolumn{2}{|c|}{ C SETUP PARTON NUMBERS } \\
\hline \multicolumn{2}{|r|}{ 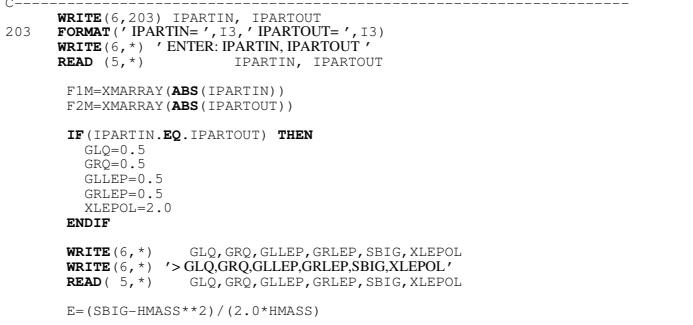 } \\
\hline \multicolumn{2}{|c|}{ C SET THE LOOP } \\
\hline & 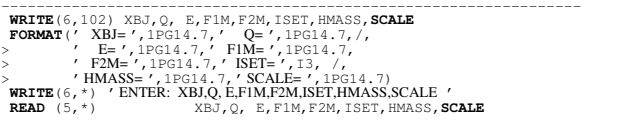 \\
\hline $\mid \begin{array}{ll}C--1 \\
C\end{array}$ SET & THE SCALES \\
\hline & \\
\hline
\end{tabular}

Figure E10: Program which computes each diagram; pages 17-20. 

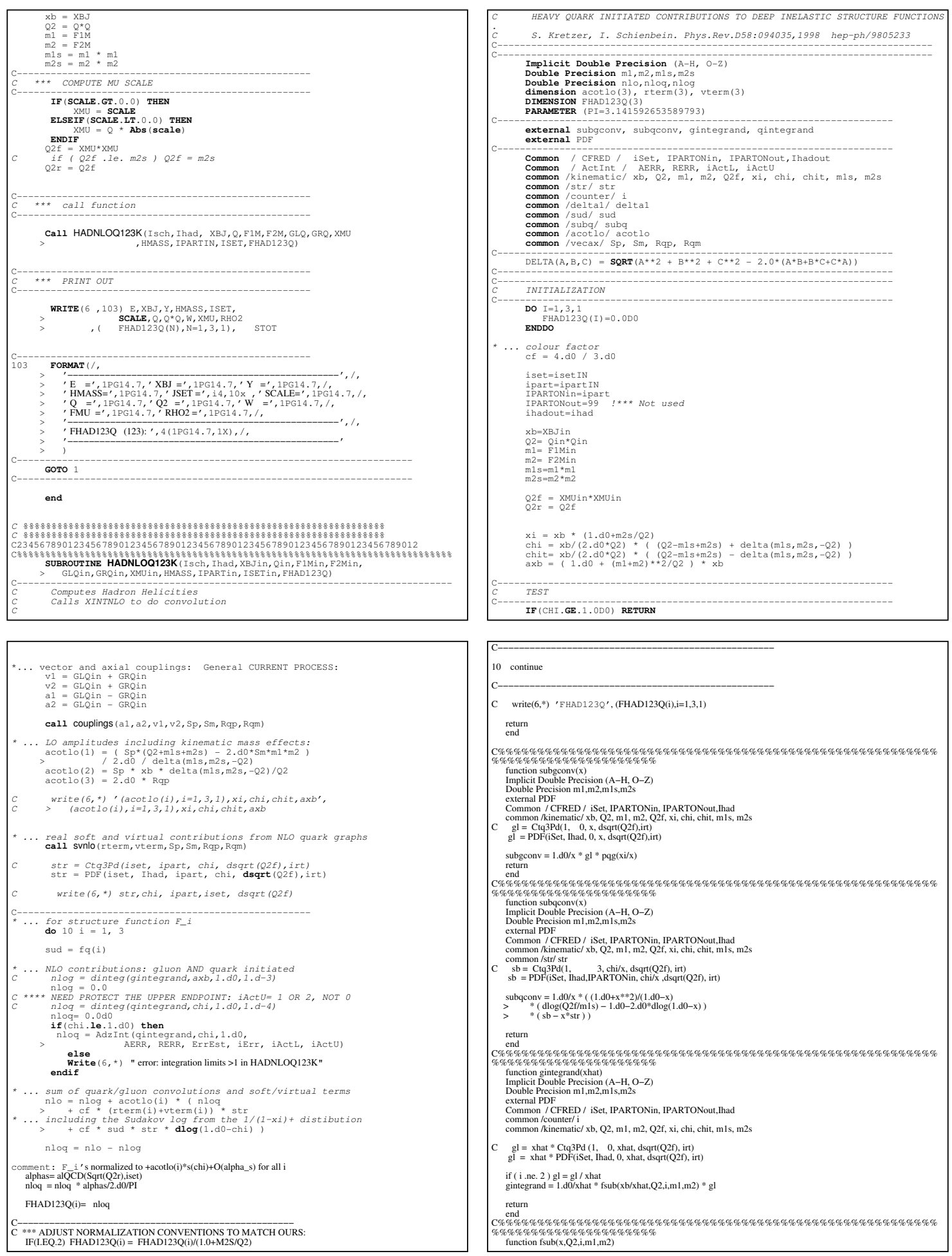

Figure E11: Program which computes each diagram; pages 21-24. 

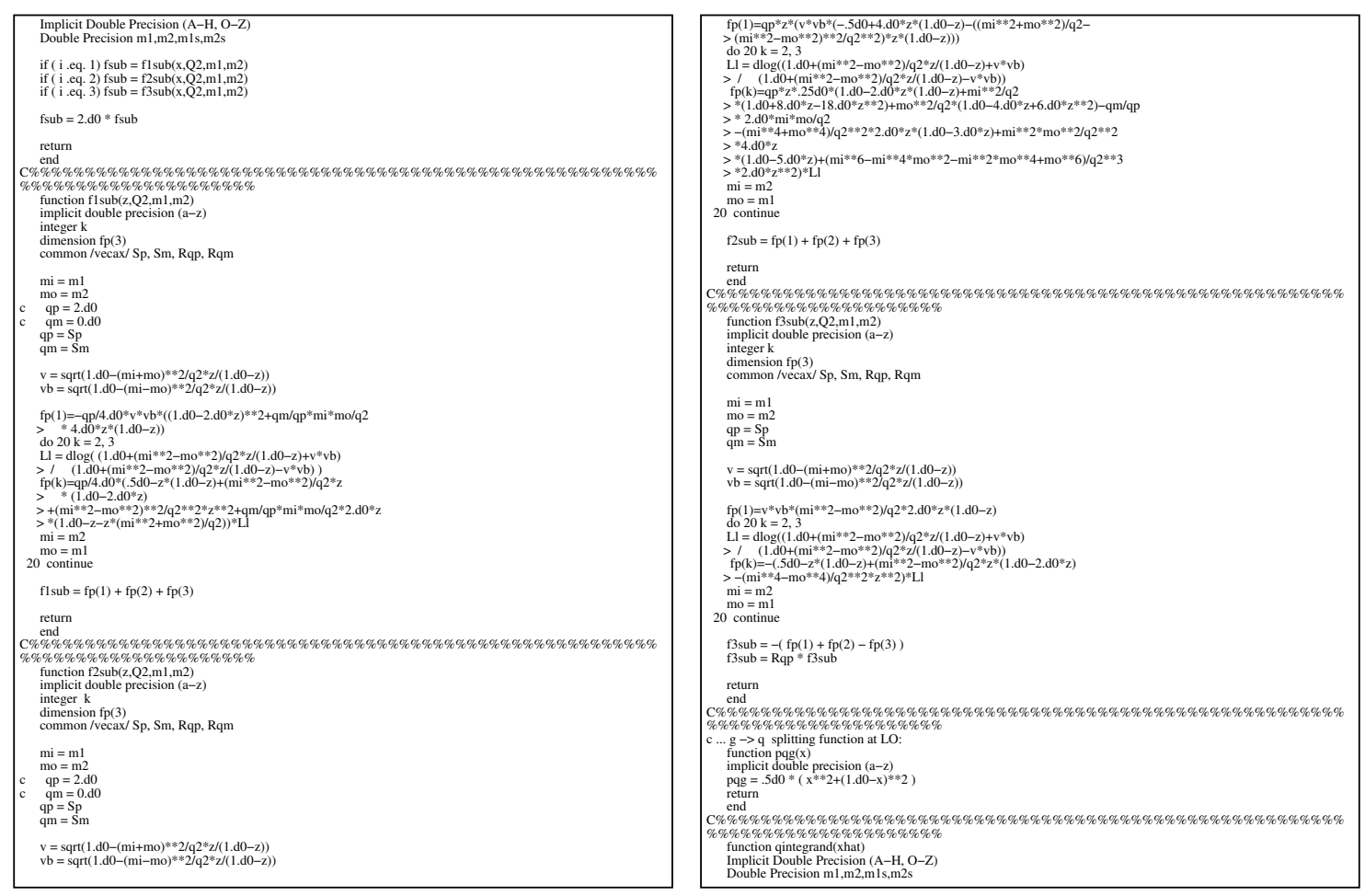

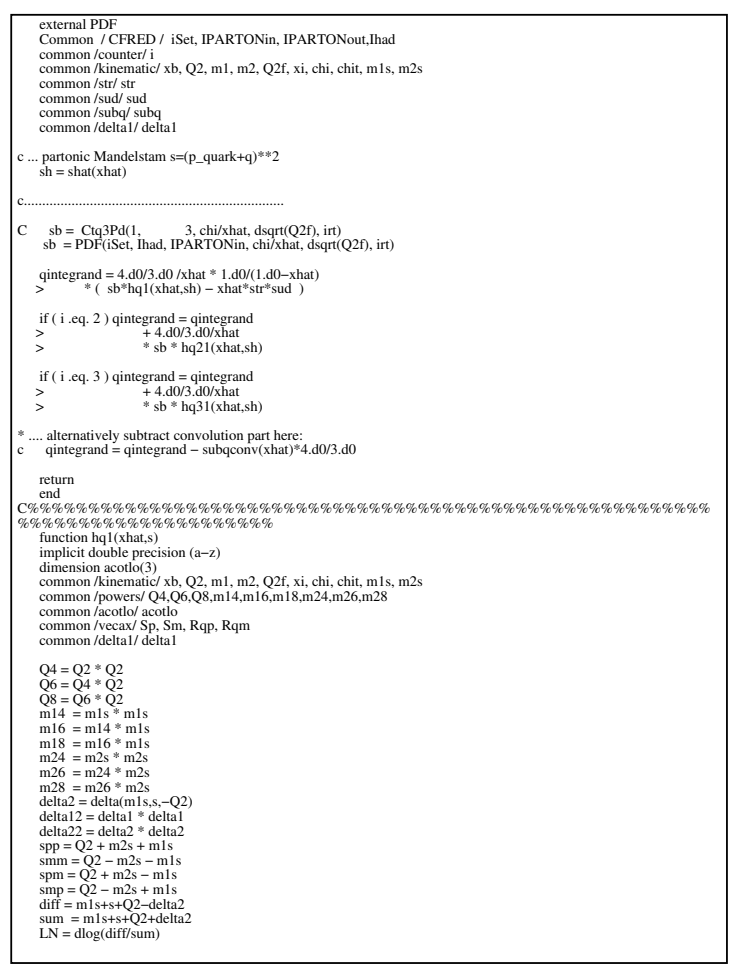

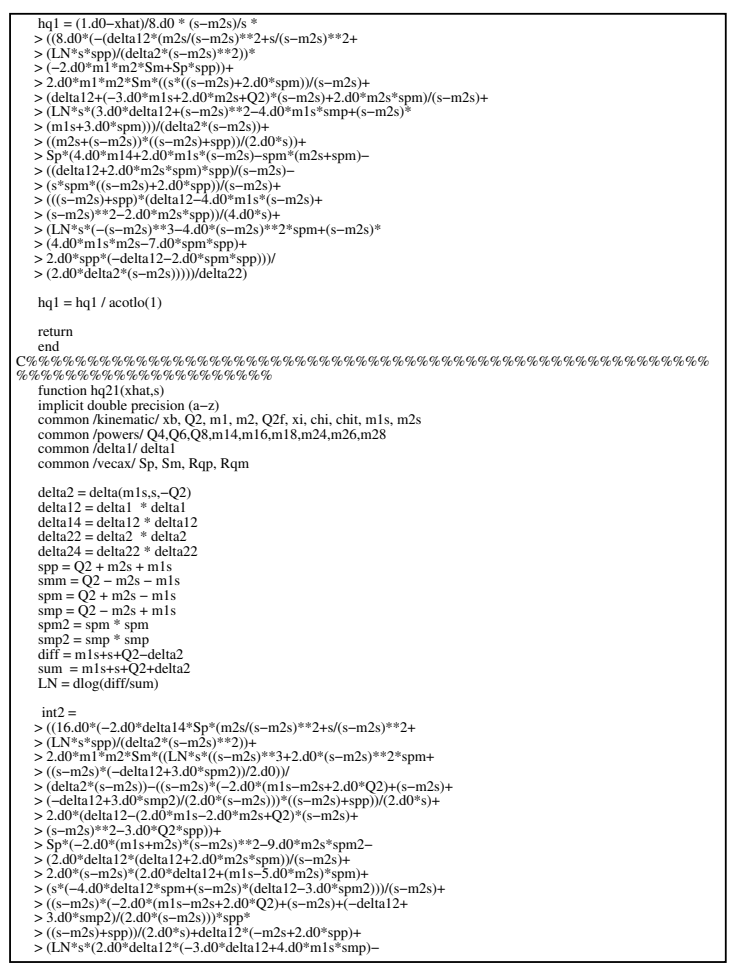

Figure E12: Program which computes each diagram; pages 25-28. 




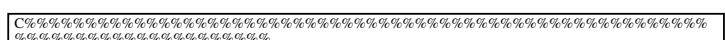

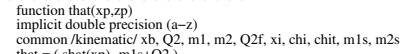

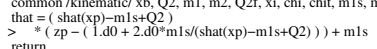

retur
end

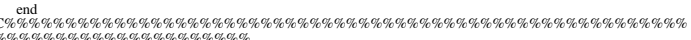

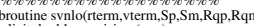

implicit double precision (a-z)
INTEGER iErr, iActL, iActu
dimension rterm(3), vterm(3)

dimension rerm( $(3)$, verm(3)
external helpf

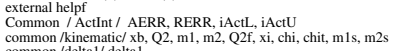

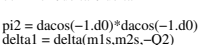

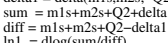

arguments of dilogs as
$\mathrm{lb} 1=2 . \mathrm{d} 0 *$ *delta1//sum
$\mathrm{lb} 2=-$ diff $/ 2$.do/delta 1

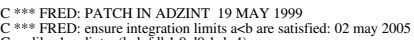

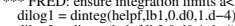

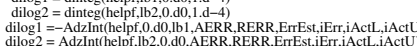

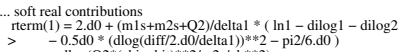

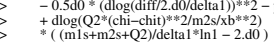

.... app is ml $1 \rightarrow 0$ limit of $\mathrm{rterm}$
app $=(\mathrm{dlog}((\mathrm{Q} 2+\mathrm{m} 2 \mathrm{~s}) * * 2 / \mathrm{m} 2 \mathrm{~s} / \mathrm{m} 1 \mathrm{~s})-2 . \mathrm{d} 0) * \operatorname{dog}((\mathrm{Q} 2+\mathrm{m} 2 \mathrm{~s}) * * 2 / \mathrm{Q} 2 / \mathrm{m} 2 \mathrm{~s})$



write( $(6, *) \operatorname{rterm}(1)$
$\operatorname{rterm}(2)$
$\operatorname{riterm}(1)$

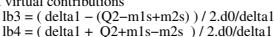

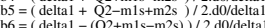

23456789012345678901234567890123456789012345678901234567890123456789012

**** FRED: ensure integration limits a 4 a a re satisfied: 02 may 2005

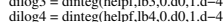

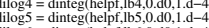
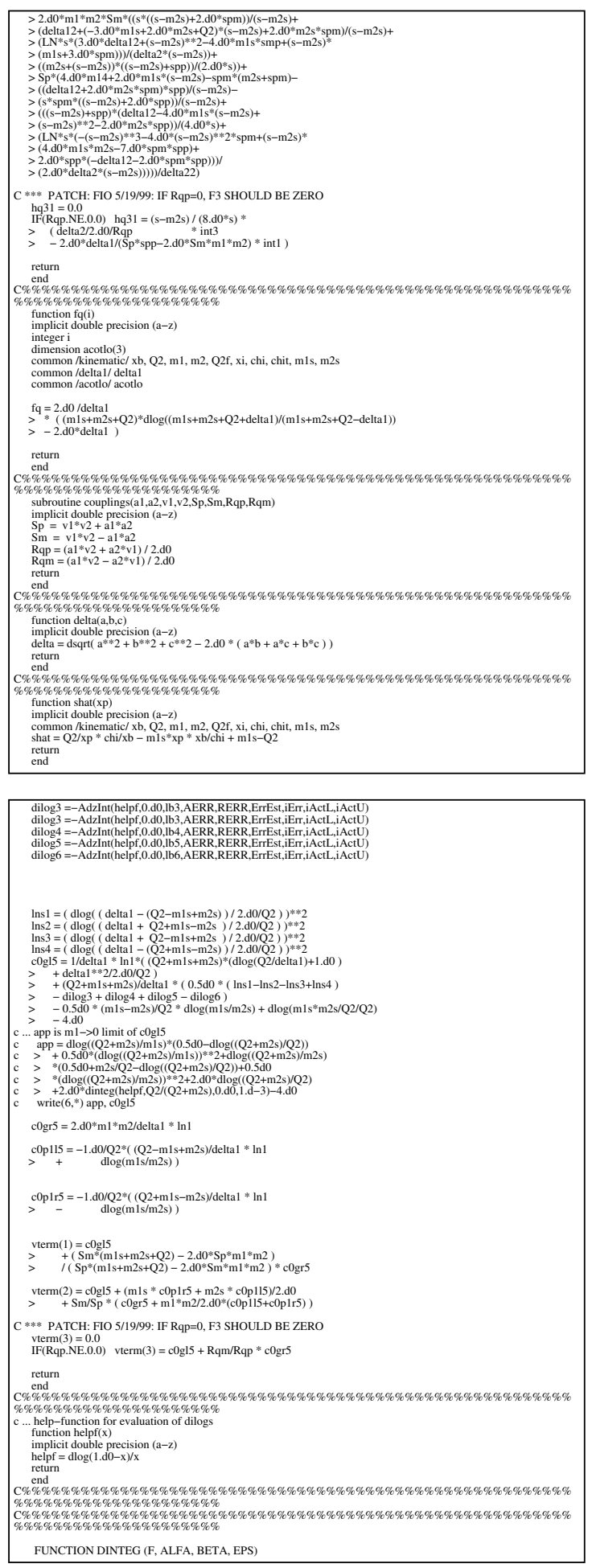

Figure E13: Program which computes each diagram; pages 29-32. 


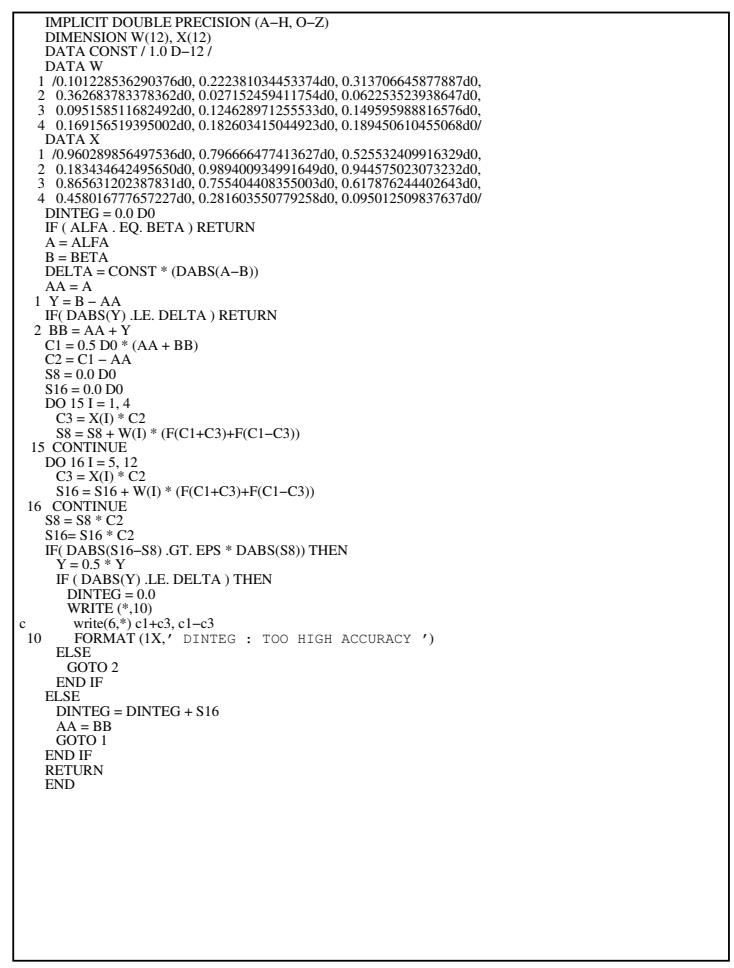

Figure E14: Program which computes each diagram; page 33. 


\title{
APPENDIX F
}

\author{
FIT RESULTS
}

\section{F.1 CORRELATION TABLES}

\section{F.1.1 Non-Singlet Fit}

Table F1 shows the correlations among fit parameters from non-singlet fit results using ACOT model. Table F2 shows the correlations among fit parameters from non-singlet fit

Table F1: The correlation matrix for non-singlet fit. ACOT model.

\begin{tabular}{|c|rrrr|}
\hline \hline Parameter & $\Lambda_{Q C D}$ & $A 1_{u_{v}}$ & $A 2_{u_{v}}$ & norm \\
\hline$\Lambda_{Q C D}$ & 1.000 & -0.285 & -0.594 & -0.777 \\
$A 1_{u_{v}}$ & -0.285 & 1.000 & 0.813 & 0.728 \\
$A 2_{u_{v}}$ & -0.594 & 0.813 & 1.000 & 0.717 \\
norm & -0.777 & 0.728 & 0.717 & 1.000 \\
\hline \hline
\end{tabular}

results using $\overline{M S}$ model. 
Table F2: The correlation matrix for non-singlet fit. $\overline{M S}$ model.

\begin{tabular}{|c|cccc|}
\hline \hline Parameter & $\Lambda_{Q C D}$ & $A 1_{u_{v}}$ & $A 2_{u_{v}}$ & norm \\
\hline$\Lambda_{Q C D}$ & 1.000 & -0.283 & -0.596 & -0.779 \\
$A 1_{u_{v}}$ & -0.283 & 1.000 & 0.812 & 0.725 \\
$A 2_{u_{v}}$ & -0.596 & 0.812 & 1.000 & 0.717 \\
norm & -0.779 & 0.725 & 0.717 & 1.000 \\
\hline \hline
\end{tabular}

\section{F.1.2 Combined Fit}

Table F3 shows the correlations among fit parameters from combined fit results using $A C O T$ model. Table F4 shows the correlations among fit parameters from combined fit results using

Table F3: The correlation matrix for combined fit. ACOT model.

\begin{tabular}{|c|rrrrrrr|}
\hline \hline Parameter & $\Lambda_{Q C D}$ & $A 1_{u_{v}}$ & $A 2_{u_{v}}$ & $A 0_{u d}$ & $A 2_{u d}$ & $A 2_{g}$ & norm \\
\hline$\Lambda_{Q C D}$ & 1.000 & -0.037 & -0.293 & -0.238 & -0.008 & -0.348 & -0.436 \\
$A 1_{u_{v}}$ & -0.037 & 1.000 & 0.888 & 0.774 & 0.584 & -0.148 & 0.807 \\
$A 2_{u_{v}}$ & -0.293 & 0.888 & 1.000 & 0.629 & 0.536 & -0.049 & 0.709 \\
$A 0_{u d}$ & -0.238 & 0.774 & 0.629 & 1.000 & 0.680 & 0.242 & 0.807 \\
$A 2_{u d}$ & -0.008 & 0.584 & 0.536 & 0.680 & 1.000 & 0.122 & 0.283 \\
$A 2_{g}$ & -0.348 & -0.148 & -0.049 & 0.242 & 0.122 & 1.000 & 0.016 \\
$n$ orm & -0.436 & 0.807 & 0.709 & 0.807 & 0.283 & 0.016 & 1.000 \\
\hline \hline
\end{tabular}

$\overline{M S}$ model. All experimental errors taken into account. 
Table F4: The correlation matrix for combined fit. $\overline{M S}$ model.

\begin{tabular}{|c|rrrrrrr|}
\hline \hline Parameter & $\Lambda_{Q C D}$ & $A 1_{u_{v}}$ & $A 2_{u_{v}}$ & $A 0_{u d}$ & $A 2_{u d}$ & $A 2_{g}$ & norm \\
\hline$\Lambda_{Q C D}$ & 1.000 & 0.114 & -0.215 & -0.449 & -0.072 & -0.652 & -0.426 \\
$A 1_{u_{v}}$ & 0.114 & 1.000 & 0.868 & 0.573 & 0.574 & -0.246 & 0.746 \\
$A 2_{u_{v}}$ & -0.215 & 0.868 & 1.000 & 0.568 & 0.563 & -0.004 & 0.699 \\
$A 0_{u d}$ & -0.449 & 0.573 & 0.568 & 1.000 & 0.650 & 0.517 & 0.797 \\
$A 2_{u d}$ & -0.072 & 0.574 & 0.563 & 0.650 & 1.000 & 0.132 & 0.337 \\
$A 2_{g}$ & -0.652 & -0.246 & -0.004 & 0.517 & 0.132 & 1.000 & 0.161 \\
$n$ orm & -0.426 & 0.746 & 0.699 & 0.797 & 0.337 & 0.161 & 1.000 \\
\hline \hline
\end{tabular}

\section{F.2 NUCLEAR PDFS FROM FIT TO NUTEV DATA}

Figures F1, F2 show the shape of the PDF distributions as result of our QCD fits (ACOT and $\overline{M S}$ models). 




Figure F1: Nuclear PDFs at $Q_{0}^{2}=5 \mathrm{GeV}^{2}$. ACOT scheme.

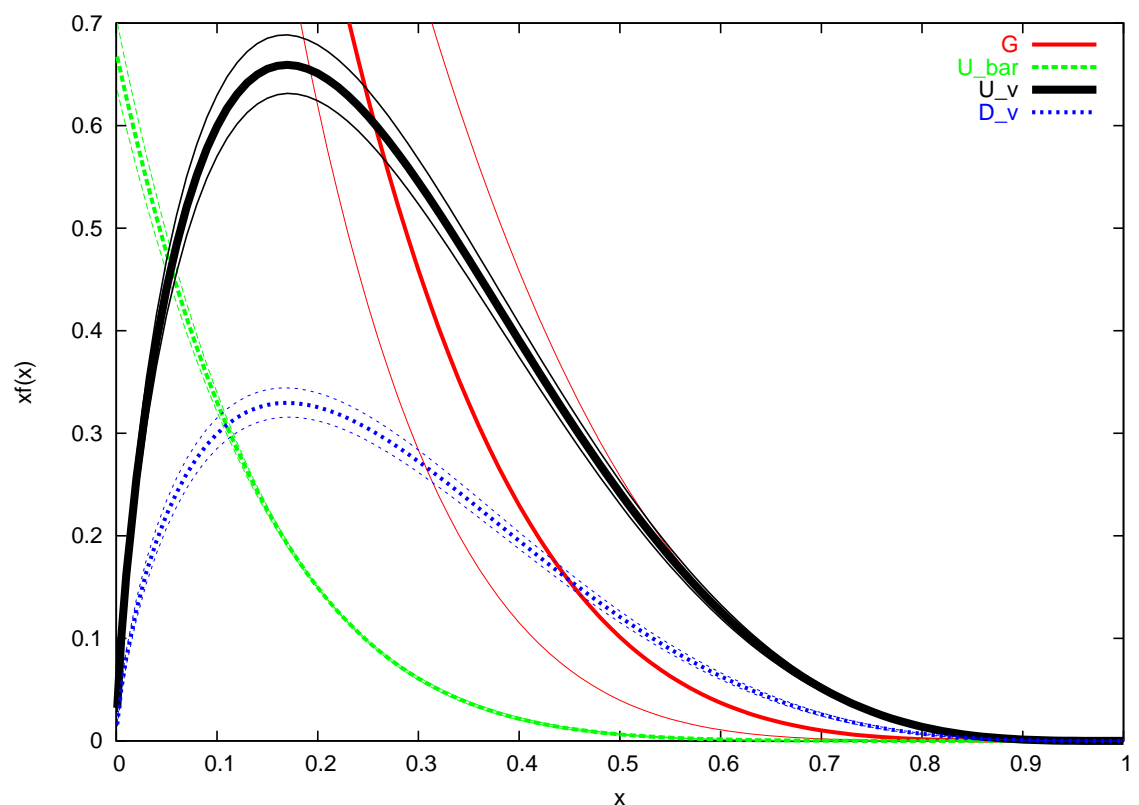

Figure F2: Nuclear PDFs at $Q_{0}^{2}=5 \mathrm{GeV}^{2} . \overline{M S}$ scheme. 


\section{APPENDIX G}

\section{TESTS OF THE QCD PROGRAM}

To ensure consistency of the QCD program checks were performed.

\section{G.1 TEST OF MINIMIZATION USING CCFR DATA}

A way to test the minimization of the NuTeV analysis is by comparing the quoted CCFR results in Ref. [50] to the results produced using CCFR data and NuTeV QCD program. The CCFR data used in this study is taken from Durham database, which correspond to the tables used in [50]:

- CCFR $x F_{3}$ : Phys. Rev. Lett. 79: 1213 (1997) for the Non-Singlet Fit;

- CCFR $F_{2}$ and $x F_{3}$ : Nevis Report 292 (1996) (Bill Seligman Ph.D Thesis).

The output is presented in the table G1. For testing purposes only statistical errors were used. Also, we check the results by fixing the fit parameters to the final CCFR fit results as quoted from Ref. [50] (see Table G2).

We have used the following cuts to be consistent with Ref. [50]:

- $x<0.7, Q^{2}>5 \mathrm{GeV}^{2}, m_{b}=4.5 \mathrm{GeV}, m_{c}=1.5 \mathrm{GeV}$. 
Table G1: Test of minimization using CCFR data

\begin{tabular}{|c||c|c||c|c|}
\hline \hline \multicolumn{1}{|c||}{} & \multicolumn{2}{c||}{$x F_{3}$ fit } & \multicolumn{2}{c|}{$x F_{3}$ and $F_{2}$ fit } \\
& CCFR fitter & NuTeV fitter & CCFR fitter & NuTeV fitter \\
\hline$\Lambda^{\left(n_{f}=4\right)}(\mathrm{MeV})$ & $387 \pm 42$ & $364 \pm 43$ & $381 \pm 24$ & $392 \pm 24$ \\
\hline$A_{1 u_{v}} \equiv \eta_{1}$ & $0.801 \pm 0.010$ & $0.807 \pm 0.010$ & $0.804 \pm 0.008$ & $0.81 \pm 0.01$ \\
$A_{2 u_{v}} \equiv \eta_{2}$ & $3.89 \pm 0.03$ & $3.90 \pm 0.04$ & $3.91 \pm 0.02$ & $3.89 \pm 0.02$ \\
$A_{o u_{v}}+A_{0 d_{v}} \equiv A_{N S}$ & $8.57 \pm 0.20$ & $6.25+3.12$ & $8.65 \pm 0.16$ & $3.06+6.13$ \\
$2 A_{0 u_{d}} \equiv A_{S}$ & & & $1.52 \pm 0.02$ & $2 \times(0.81 \pm 0.01)$ \\
$A_{2 u_{d}} \equiv \eta_{S}$ & & & $7.82 \pm 0.09$ & $7.68 \pm 0.01$ \\
$A_{0 g} \equiv A_{G}$ & & & $1.94 \pm 0.24$ & 2.13 \\
$A_{2 g} \equiv \eta_{G}$ & & & $4.44 \pm 0.65$ & $4.47 \pm 0.70$ \\
\hline$\chi^{2} / d o f$ & $81.4 / 82$ & $87.56 / 82$ & $190.6 / 164$ & $199 / 165$ \\
\hline \hline
\end{tabular}

Table G2: Fit results using CCFR data with parameters fixed to the values quoted from Ref. [50]

\begin{tabular}{|l||c|c||c|c|}
\hline \hline \multicolumn{1}{|c||}{} & \multicolumn{2}{|c||}{$x F_{3}$ fit } & \multicolumn{2}{c|}{$x F_{3}$ and $F_{2}$ fit } \\
& CCFR fitter & NuTeV fitter & CCFR fitter & NuTeV fitter \\
\hline$\Lambda^{\left(n_{f}=4\right)}(\mathrm{MeV})$ & $387 \pm 42$ & $398 \pm 8$ & $381 \pm 24$ & $378 \pm 5$ \\
\hline \hline
\end{tabular}

Conclusions:

- The results agree within errors.

- It is claimed in Ref. [50] that the QCD constraints would shift the results up by about $15 \mathrm{MeV}$. 


\section{G.1.1 CCFR vs NuTeV}

For a more consistent way in comparing the CCFR results to the $\mathrm{NuTeV}$ one should compare the CCFR data (unfortunately only the $F_{2}$ has been extracted) from:

- UnKi Yang $F_{2}$ data: Phys.Rev.Lett.86:2742-2745 (2001) [63]

The extraction of this data set is performed in a similar fashion as the method used by $\mathrm{NuTeV}$.

- compare results using $F_{2}$ from UnKi Yang (CCFR) to $F_{2}$ from NuTeV. The errors are added in quadrature. See Table G3.

Table G3: Comparison of fit results using CCFR data [63].

\begin{tabular}{|c||c|c|}
\hline \hline \multicolumn{1}{|c||}{} & \multicolumn{2}{c|}{$F_{2}$ only } \\
& UnKi data & NuTeV data \\
\hline$\Lambda^{\left(n_{f}=4\right)}(\mathrm{MeV})$ & $330 \pm 64$ & $425 \pm 71$ \\
\hline$A_{1 u_{v}} \equiv \eta_{1}$ & $0.804 \pm 0.047$ & $0.762 \pm 0.004$ \\
$A_{2 u_{v}} \equiv \eta_{2}$ & $4.02 \pm 0.08$ & $3.67 \pm 0.08$ \\
$A_{o u_{v}}+A_{0 d_{v}} \equiv A_{N S}$ & $6.22+3.11$ & $5.26+2.63$ \\
$2 A_{0 u_{d}} \equiv A_{S}$ & $2 \times(0.73 \pm 0.07)$ & $2 \times(0.72 \pm 0.06)$ \\
$A_{2 u_{d}} \equiv \eta_{S}$ & $9.24 \pm 0.69$ & $8.20 \pm 0.68$ \\
$A_{0 g} \equiv A_{G}$ & 2.78 & 2.58 \\
$A_{2 g} \equiv \eta_{G}$ & $5.18 \pm 1.86$ & $5.00 \pm 1.86$ \\
\hline \hline$\chi^{2} / d o f$ & 0.52 & 0.86 \\
\hline \hline
\end{tabular}

- known differences: CCFR (UnKi) data is from a 2-parameter fit, $\mathrm{NuTeV}$ data is from 1-parameter fit. The difference between $1 \mathrm{p}$ and $2 \mathrm{p}$ fits are known to matter only in the lowest $x$ bins, thus we compare the results for $x>0.1$ as well (see Table G4). 
Table G4: Comparison between fit results using CCFR (UnKi) and NuTeV data for $x>0.1$

\begin{tabular}{|c||c|c|}
\hline \hline \multicolumn{1}{|c||}{} & \multicolumn{2}{c|}{$F_{2}$ only } \\
& UnKi data & NuTeV data \\
\hline$\Lambda^{\left(n_{f}=4\right)}(\mathrm{MeV})$ & $322 \pm 64$ & $454 \pm 103$ \\
\hline$A_{1 u_{v}} \equiv \eta_{1}$ & $0.743 \pm 0.086$ & $0.665 \pm 0.123$ \\
$A_{2 u_{v}} \equiv \eta_{2}$ & $3.96 \pm 0.09$ & $3.41 \pm 0.35$ \\
$A_{o u_{v}}+A_{0 d_{v}} \equiv A_{N S}$ & $5.22+2.61$ & $3.85+1.92$ \\
$2 A_{0 u_{d}} \equiv A_{S}$ & $2 \times(0.66 \pm 0.10)$ & $2 \times(0.55 \pm 0.10)$ \\
$A_{2 u_{d}} \equiv \eta_{S}$ & $9.87 \pm 2.19$ & $5.33 \pm 2.99$ \\
$A_{0 g} \equiv A_{G}$ & 3.50 & 2.34 \\
$A_{2 g} \equiv \eta_{G}$ & $6.07 \pm 2.68$ & $4.30 \pm 2.98$ \\
\hline \hline$\chi^{2} /$ dof & 0.55 & 0.84 \\
\hline \hline
\end{tabular}

- We notice $<2 \sigma$ discrepancy between $\mathrm{NuTeV}$ and CCFR Singlet results. This is purely due to the differences in the data between CCFR and NuTeV.

\section{G.2 TEST OF THE FITTER WITH CORRELATIONS}

This is easily checked by setting all the correlations to zero and expect to reproduce the uncorrelated results. This has been successfully tested both for non-singlet and combined fits. The comparisons gave identical results as it was expected and thus the check of the correlation has been completed. 


\section{G.3 TEST OF THE FUSION}

\section{G.3.1 Fake data}

1. generate a fake data file with structure functions calculated with a known set of PDFs (i.e. CTEQ4HQ);

2. insert the fake data file in the QCD fit (i.e. use it as real data);

3. compare the output with the input. $\left(\Lambda_{Q C D}\right.$ quoted by the CTEQ4HQ group, obtained using the same code to calculate structure functions).

The global fits for the CTEQ4HQ, at 4 flavors, in the ACOT were performed for $0.01<x<$ $0.7, Q 2>4$ (see Table G5). The NS fit results of the fake data study: The fake data study

Table G5: Fake Data study using ACOT scheme.

\begin{tabular}{|l|c|}
\hline \hline pdf & $\Lambda^{\left(n_{f}=4\right)}(\mathrm{MeV})$ \\
\hline \hline CTEQ4HQ & 298 \\
\hline Fake data & 301 \\
\hline \hline
\end{tabular}

closes within 1\%. Thus, it validates the fitter and the success of the fusion of the packages.

\section{G.3.2 Comparison between Massless $\overline{M S}$ Models}

The very nice feature of this QCD program is its access to different theory models. Therefore, it provides direct comparisons among various calculations. For example, one can compare calculation of J. Owens of the structure functions based on the massless $\overline{M S}$ scheme [53] to calculation of F. Olness using the massless $\overline{M S}$ [54]. The comparisons between these two calculations are shown in Table G6. The calculation of the structure functions agree within $1 \%$ providing another good test of the fusion of QCD programs as well as a check of the massless prediction viewed from two different perspectives. 
Table G6: Comparisons between two $\overline{M S}$ models for the NS and Combined fits.

\begin{tabular}{|c||c|c|}
\hline \hline Program basis & $\begin{array}{c}x F_{3} \text { fit } \\
\Lambda_{f}^{\left(n_{f}=4\right)}(\mathrm{MeV})\end{array}$ & $\begin{array}{c}x F_{3} \text { and } F_{2} \text { fit } \\
\Lambda_{f}^{\left(n_{f}=4\right)}(\mathrm{MeV})\end{array}$ \\
\hline \hline Fred Olness & $476 \pm 60$ & $439 \pm 58$ \\
\hline Jeff Owens & $476 \pm 60$ & $433 \pm 51$ \\
\hline \hline
\end{tabular}

\section{G.3.3 ACOT Technicalities}

The factorization theorem is conveniently expressed as a composition of t-channel 2PI amplitudes,

$$
F=\sum_{N=1}^{\infty} C(K)^{N} T+\text { Non factorizable contributions, }
$$

where $C$ represents a graph for a hard scattering (hard scattering Coefficients), $K$ a graph for a rung (Kernel), $T$ represents a graph that couples to the target (Target). The idea of

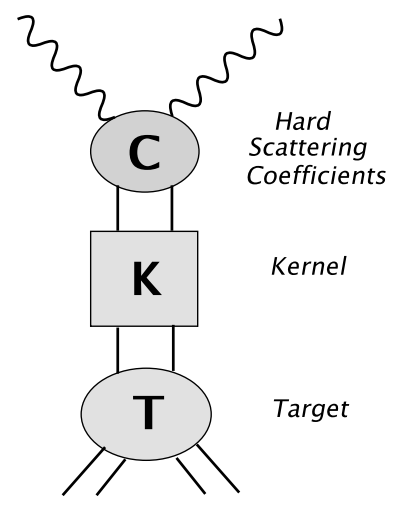

Figure G1: Decomposition of the hadronic structure function in terms of 2PI amplitudes

ACOT scheme is to reorganize the infinite sum by introducing a collinear projector operator $Z$ which basically connects the hard scattering terms to the PDFs. Hence, the main point to generalize the factorization theorem to heavy quarks masses is to construct properly this 
projector operator (full details could be found in [8]).

$$
F \approx \underbrace{C\left[\frac{1}{1-(1-Z) K}\right]}_{\text {hard scatt. coeff.(all orders) }} Z \underbrace{\left[\frac{1}{1-K} T\right]}_{\text {pdf's }}+\underbrace{O\left(\frac{\Lambda_{Q C D}^{2}}{Q^{2}}\right)}_{\text {Non-factorizable contributions }}
$$

This relation is valid to all-orders.

\section{G.4 ACOT AND ITS VARIANT}

- FULL ACOT scheme: Full ACOT scheme is designed to work for quarks with finite masses, in particular it is used for the case when only heavy quarks are involved. Figure G2 displays each of the contributing diagrams.

$$
H A D \_L O-H A D \_S U B+H A D \_N L O \_K r-H A D \_S U B \_Q+H A D \_N L O \text {. }
$$
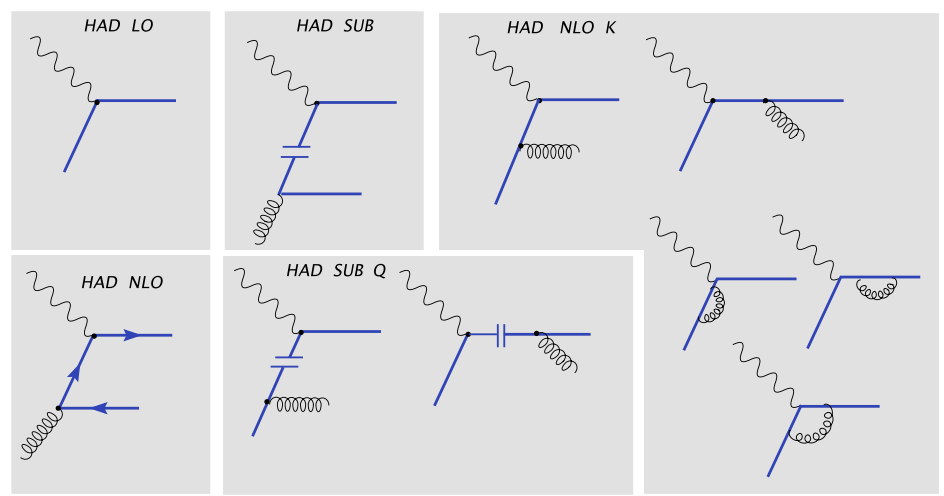

Figure G2: Full ACOT scheme with $m_{c} \neq 0$ 
- Shortcut $A C O T$ : This variant was designed for the structure functions with both light and heavy quarks contributions. Figure G3 displays each of the contributing diagrams. It is numerically more stable when dealing with the light quark parts because proper cancellations then occur.

$$
H A D \_L O-H A D \_S U B+H A D \_N L O \_Q\left(M_{H}=0\right)+H A D \_N L O \text {. }
$$
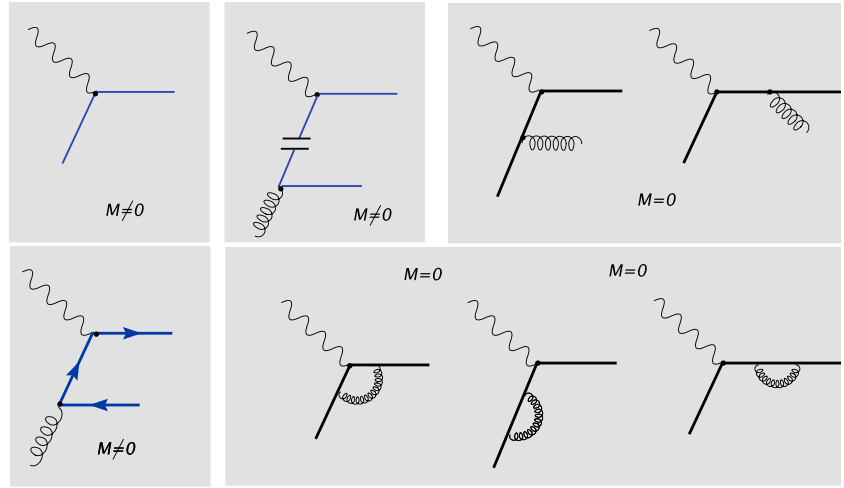

Figure G3: Shortcut ACOT scheme. 


\section{BIBLIOGRAPHY}

[1] SLAC-MIT collaboration, G. Miller et al., Phys. Rev. D5 528 (1972).

[2] J. D. Bjorken, Phys. Rev. 179, 1547 (1969).

[3] C. G. Callan and D. Gross, Phys. Rev. Lett. 22, 156 (1969).

[4] Particle Data Group Listing (2005).

[5] D. E. Soper, J. C. Collins and G. Sterman, Factorization of Hard Processes in QCD, Singapore: World Scientific (1989).

[6] G. Dissertori, I. Knowles and M. Schmelling, "Quantum Chromodynamics, High Energy Experiments and Theory" (2003).

[7] W. A. Bardeen, A. J. Buras, D. W. Duke, T. Muta, Phys. Rev. D18 3998 (1978).

[8] J. C. Collins, Phys. Rev. D58, 094002 (1998).

[9] Heavy Quark Production and PDF subgroup report; hep-ph/0005112

[10] M. Gluck, E. Hoffmann, and E. Reya, Z. phys. C13, 119 (1982);

M. Gluck, E. Hoffmann, and E. Reya, Z. phys. C38, 441 (1988); erratum: ibid., C39, 590 (1988); E. Laenen, Nucl. Phys. B392, 162 (1993)

[11] J. C. Collins, Phys. Rev. D58, 094002 (1998);

M. A. .G. Aivazis, F. .I. Olness, and W. K. Tung,Phys. Rev. Lett.65,2339 (1990);

M. A. G. Aivazis, J. C. Collins, F. I. Olness and W. K. Tung, Phys. Rev. D50,3102 (1994);

B. .A. Kniehl, M. Kramer, G. Kramer, and M. Spira, Phys. Lett. B356,539 (1995);

M. .Buza, Y. Matiounine, J. Smith, and W. L. van Neerven, Eur. Phys. J. C1,301 (1998);

R. S. Thorne and R. G .Roberts, Phys. Rev D57,6871 (1998);

R. S. Thorne and R. G .Roberts, Phys. Lett B421,303 (1998); 
[12] M. A. G. Aivazis, J. C. Collins, F. I. Olness and W. K. Tung, Phys. Rev. D50, 3085, 3102 (1994).

[13] Yu. L. Dokshitzer, Sov.Phys.JETP46 (1977);

V. N. Gribov and L. N. Lipatov, Sov.J.Nucl.Phys 15, 438 (1972);

L. N. Lipatov, Sov.J.Nucl.Phys 20, 95 (1975);

G. Altarelli and G. Parisi Nuc.Phys B126, 298 (1977).

[14] F. I. Olness, R. J. Scalise, Phys. Rev. D57(1) (1998).

[15] H. Georgi and H. D. Politzer, Phys. Rev. D14 1829 (1976).

[16] S. Kretzer, H. Reno, Phys. Rev. D69 , 034002 (2002).

[17] R. G. Arnold et al., Phys. Rev. Lett. 52, 727 (1984);

J. Gomez et al., Phys.Rev D49, 4348 (1994).

[18] G. Amaudruz et al., Nucl. Phys. B441, 3 (1995).

[19] M. .R. Adams et al., Zeit. Phys C67, 403 (1995).

[20] S. A. Kulagin, R. Petti [hep-ph/0412425].

[21] J. W. Qiu and I. Vitev, Phys. Lett. B587, 52 (2004).

[22] M. Tzanov et al. (NuTeV Collaboration), Phys. Rev. D74 012008 (2006);

M. Tzanov, Ph.D. Thesis, University of Pittsburgh (2005).

[23] G. P. Zeller, Ph. D. Thesis, Northwestern University (2002).

[24] A. G.Vaitaitis, Ph. D. Thesis, Columbia University, Nevis 298 (2000).

[25] S. Avvakumov, Ph. D. Thesis, University of Rochester (2003).

[26] D. Mason, Ph. D. Thesis, University of Oregon (2005).

[27] J. A. Formaggio, Ph. D. Thesis, Columbia University (2001).

[28] D. A. Harris et al. (NuTeV Collaboration), Nucl. Instrum. Methods A 447, 377 (2000).

[29] C. W. Fabjan and T. Ludlam, Ann. Rev. Nucl. Part. Sci. 32, 335 (1982).

[30] ANSYS, Finite element analysis software [http://www.ansys.com].

[31] R. H. Bernstein et al., FERMILAB-TM-1884, (1994); 
J. Yu et al., FERMILAB-TM-2040 (1998).

[32] L. F. Landau, J.Phys. USSR 8:2001 (1994).

[33] J. Conrad, M. H. Shaevitz, and T. Bolton, Rev. Mod. Phys. 704 (1998).

[34] G. Sterman et al., Rev. Mod. Phys. 67157 (1995).

[35] T. A. Gabriel and P. E. Groom, Nucl. Instr. Meth. A338, 336 (1994).

[36] GEANT Detector Description and Simulation Tool, CERN Program Library Long Writeup W5013.

[37] G. Ingelman, A. Esin, J. Rathsman, Computer Physics Communications 101, 108-134 (1997).

[38] A. J. Buras and K. J. F. Gemers, Nucl. Phys. B132,249 (1978).

[39] S. Rabinowitz et al. (CCFR collaboration), Phys. Rev. Lett. 70 , 134 (1993).

[40] A. Hawker, et al. Phys. Rev. Lett. 80, 3715 (1998).

[41] M. Gluck et al. (GRV collaboration),Z. Phys. C67 433 (1995).

[42] A. Bodek and U. K. Yang, Nucl. Phys. Proc. Suppl. 11270 (2002).

[43] L. W. Whitlow et al., Phys. Lett. B282 475 (1992).

[44] A. C. Benvenuti et al., Phys. Lett. B223 485 (1989);

A. C. Benvenuti et al., Phys. Lett. B236 592 (1989).

[45] M. Arneodo et al., Nucl. Phys. B483 3 (1997).

[46] L. W. Whitlow et al., Phys. Lett. B250, 193 (1990).

[47] D. Y. Bardin and V. A. Dokuchaeva, JINR-E2-86-260 (1986).

[48] P. Villain et al., Eur. Phys. J. C11 19 (1999);

P. Astier et al., Phys. Lett. B486 35 (2000).

[49] R. M. Barnet, Phys. Rev D14,70 (1976).

[50] W. Seligman, Ph. D. Thesis, Columbia University, Nevis 292 (1997).

[51] W. Seligman et al., Phys. Rev. Lett. 79,1213 (1997); 
[52] J. F. Owens, Wu-Ki Tung, Ann. Rev. Nucl. Part. Sci. 42:291-332 (1992).

[53] J. F. Owens, private communication;

A. Devoto, D. W. Duke, J. F. Owens, R. G. Roberts, Phys. Rev. D27508 (1983).

[54] F. I. Olness, private communication;

M. A. G. Aivazis, J. C. Collins, F. I. Olness and W. K. Tung, Phys. Rev. D50, 3085, 3102 (1994).

[55] Numerical Recipes in Fortran 77 [http://library.lanl.gov/numerical/bookfpdf.html].

[56] P. R. Bevington, D. Keith Robinson, "Data reduction and error analysis for the physical sciences" (1992).

[57] M. Virchaux and A. Milsztajn, Phys. Lett. B 274, 221 (1992).

[58] S. Alekhin, JHEP 0302, 015 (2003).

[59] A. D. Martin, R. G. Roberts, W. J. Stirling, and R. S.Thorne, Phys. Lett., B604, 61 (2004).

[60] J. Kim et al.(CCFR collaboration),Phys. Rev. Lett. 81, 3595 (1998).

[61] J. Blumlein and H. Bottcher, Nucl. Phys. B636, 225 (2002).

[62] P. L. Anthony et al., Phys. Lett. B463, 339 (1999);

P. L. Anthony et al., Phys. Lett. B493, 19 (2000).

[63] U. K. Yang, Ph. D. Thesis, University of Rochester, UR-1583 (2001).

[64] D. Buskulic et al. (ALEPH collaboration), Phys. Lett. B357, 487 (1995); ibid., erratum Phys. Lett. B364, 247 (1995);

R. Akers et al. (OPAL collaboration), Z.Phys. C68, 203 (1995);

P. Abreu et al. (DELPHI collaboration), Phys. Lett B398, 194 (1997).

[65] T. Klugg (H1 collaboration) at DIS2005, Madison, Wisconsin (2005);

C. Adloff et al., Eur. Phys. J. C19, 289 (2001);

ZEUS Collaboration: DESY-05-019 [hep-ex/0502007].

[66] J. Kutti, V. F. Weisskopf, Phys. Rev. D4, 3418 (1971);

P. V. Landshoff and J. C. Polkinghorne, Phys. Letters 34B, 621 (1971).

[67] A. C. Benvenuti et al., Phys. Lett. B237, 592 (1990). 
[68] L. W. Whitlow, Ph. D. Thesis, SLAC-REPORT-357 (1990).

[69] R. S. Thorne and R. G. Roberts, Phys. Lett. B 421303 (1998);

A. D. Martin et al. Eur. Phys. J. C 18117 (2000).

[70] F.Halzen, A.D. Martin "Quarks and Leptons: An Introductory Course in Modern Particle Physics".

[71] Ellis, Stirling and Webber, "QCD and Collider Physics" (1996). 